Ute Nawratil

\title{
Glaubwürdigkeit in der sozialen Kommunikation
}

München 2006 
Ute Nawratil: Glaubwürdigkeit in der sozialen Kommunikation. 2. Auflage, München 2006 (digitale Ausgabe)

URL: http://epub.ub.uni-muenchen.de/archive/00000941/

Erstveröffentlichung: Westdeutscher Verlag 1997. Die vorliegende digitale Ausgabe entspricht inhaltlich der Erstausgabe. Es wurden kleinere Korrekturen vorgenommen, Seiten- und Fußnotennumerierung stimmen nicht mit der Erstausgabe überein..

Dieses Werk kann durch jedermann gemäß den Bestimmungen der Lizenz für die freie Nutzung unveränderter Inhalte genutzt werden.

Die Lizenzbedingungen können unter http://www.uvm.nrw.de/opencontent abgerufen oder bei der Geschäftsstelle des Kompetenznetzwerkes Universitätsverbund MultiMedia NRW, Universitätsstraße 11, D-58097 Hagen, schriftlich angefordert werden. 
Inhalt

1 Einleitung

2 Was ist Glaubwürdigkeit? Begriffsklärung und Definition

3 Lügen haben kurze Beine?

Die verhaltensorientierte Glaubwürdigkeitsbeurteilung ............... 22

3.1 Vorgehensweise und Befunde .................................................. 22

3.2 Zwischenbilanz.............................................................................

\section{Wer einmal lügt ...}

Die inhaltsorientierte Glaubwürdigkeitsbeurteilung ..................... 35

4.1 Glaubwürdigkeitsmerkmale des Aussageinhalts ......................... 36

4.2 Glaubwürdigkeitsmerkmale im zeitlichen Verlauf ..................... 38

4.3 Glaubwürdigkeitsmerkmale der Aussageweise ........................... 39

4.4 Persönlichkeitsmerkmale, Motive und individueller Entwicklungsstand ......................................... 41

4.5 Zwischenbilanz .................................................................

5 Wo das Wissen aufhört, fängt der Glaube an:

Die Glaubwürdigkeit der Quelle

5.1 Die Anfänge: Das 'Prestige' der Quelle

5.2 Experimente im Rahmen der Persuasionsforschung: Die Hauptdimensionen 'Kompetenz' und 'Vertrauenswürdigkeit'....

5.3 Methodische Weiterentwicklung:

Einsatz des Semantischen Differentials

5.4 Weitere Dimensionen:

Ähnlichkeit, Sympathie und Attraktivität

66

5.5 Sprechverhalten

80 
5.6 Zusätzliche Einflußfaktoren

5.6.1 Das Geschlecht des Sprechers ................................................. 85

5.6.2 Einflußfaktor Rezipient: Persönlichkeitsspezifische Merkmale.. 90

5.6.3 Einflußfaktor Rezipient: Die Beziehung zum Thema ................ 96

5.6.4 Einflußfaktor Mitteilung:

Spezielle Merkmale von Stil und Inhalt ................................... 103

5.6.5 Der Zeitpunkt der Information .................................................. 110

5.7 Der "sleeper effect" 113

5.8 Kritik an der quellenorientierten Glaubwürdigkeitsforschung ... 120

5.9 Alternative Modellvorstellungen ............................................ 125

5.10 Zwischenbilanz ........................................................................ 130

6 "Am glaubwürdigsten im Fernsehen ist immer noch

'Das Wort zum Sonntag'": Die Glaubwürdigkeit des Mediums .. 134

6.1 Eindimensionale Ansätze ....................................................... 134

6.2 Mehrdimensionale Ansätze ........................................................ 142

6.3 Spezifische Merkmale der Glaubwürdigkeit von Medien ......... 146

6.4 Zusätzliche Einflußfaktoren ..................................................... 149

6.4.1 Abhängigkeit vom Rezipienten ............................................... 149

6.4.2 Abhängigkeit vom Thema ............................................................ 151

6.5 Befunde für die Bundesrepublik Deutschland ............................. 155

6.6 Spezielle Aspekte von Medien-Glaubwürdigkeit ...................... 160

6.6.1 Stilistische und inhaltliche Elemente ........................................ 160

6.6.2 Elemente der optischen Präsentation ........................................ 161

6.7 Zwischenbilanz ....................................................................... 163

7 Vertrauenssache - Glaubwürdigkeitsprobleme in der vermittelten Kommunikation

7.1 Die Rolle der Glaubwürdigkeit im Vermittlungsund Mitteilungsprozeß

7.2 Die Information über Quellen 
7.3 Unterschiedliche Vermittlungsstrategien:

'Journalist und 'Publizist'

7.4 Die Zuschreibung von Glaubwürdigkeit als publizistische Strategie .......................................................... 185

7.5 Die Glaubwürdigkeit anonymer Quellen ................................... 193

7.6 Zwischenbilanz .................................................................... 199

8 Der politische Skandal und die Rolle der Medien .......................... 202

8.1. Definition des 'politischen Skandals' ......................................... 202

8.2. Die Rolle der Medien ............................................................. 206

8.3. Skandale und die Glaubwürdigkeit der Beteiligten ................... 211

8.4. Zwischenbilanz ..................................................................... 215

9 Zusammenfassung und Ausblick ..................................................... 216

9.1 Befunde der Glaubwürdigkeitsforschung ................................... 217

9.2 Die Glaubwürdigkeit von Akteuren:

Operationalisierung und Ergebnisse ......................................... 219

9.2.1 Kompetenz ......................................................................... 224

9.2.2 Vertrauenswürdigkeit ........................................................... 226

9.2.3 Dynamik / Selbstdarstellung ...................................................... 231

9.2.4 Soziale Billigung ................................................................... 234

9.2.5 Sympathie ............................................................................ 236

9.3 Die Berichterstattung über Skandale: Zentrale Ergebnisse ........ 238

9.4 Anwendungsmöglichkeiten .................................................... 244

Literaturverzeichnis ..................................................................... 249 


\section{Einleitung}

Warum glauben wir einigen Menschen mehr als anderen? Auf welcher Grundlage entscheiden wir, wem wir glauben können? Bei Gesprächspartnern, die wir kennen, helfen uns vorangegangene Erfahrungen. Wir messen ihre Aussagen daran, ob sie uns schon einmal - oder gar mehrmals - belogen oder uns stets die Wahrheit gesagt haben. Treffen wir auf Fremde, lassen wir uns ebenfalls von unserer Erfahrung leiten. Wir verlassen uns auf unser "Gespür" oder unsere "Menschenkenntnis". Dabei machen wir uns in der Regel nicht bewußt, daß dieses "Gefühl" auf mehr oder weniger brauchbaren Prüfkriterien basiert, die wir von Kindheit an entwickelt haben und die wir anwenden, ohne im Einzelfall jeweils konkret darüber nachzudenken. Bewußt oder unbewußt suchen wir nach Signalen oder Hinweisen, die uns Aufschluß über die Glaubwürdigkeit unseres Gegenübers geben.

Wie aber verhält es sich, wenn wir an Kommunikationsprozessen nicht direkt und unmittelbar beteiligt sind? Täglich werden wir via Massenmedien mit den unterschiedlichsten Kommunikationspartnern und ihren Aussagen konfrontiert. Häufig genug wird über Dinge geredet, die unserer eigenen Erfahrung nicht zugänglich sind. Oder es geht um Sachverhalte, von denen wir nicht genug verstehen, um abwägen zu können, welche der verschiedenen Einschätzungen oder Interpretationen, die die verschiedenen Kommunikationspartner abgeben, zutreffend sind. Wollen wir uns dennoch eine Meinung bilden oder Stellung beziehen, müssen wir einen Umweg gehen: Wenn wir die Sache nicht beurteilen können, müssen wir uns ein Urteil bilden über die Personen, die über die Sache sprechen. Nur derjenige, den wir für glaubwürdig halten, 
wird uns von seiner Sichtweise überzeugen.

Welches aber sind die Signale, auf die wir uns stützen? Und in welcher Weise sind uns diese Signale in den Massenmedien zugänglich? Mit diesen Fragen beschäftigt sich die vorliegende Arbeit. Im Anschluß an eine Begriffsklärung werden verschiedene Forschungsrichtungen betrachtet, die sich mit jeweils unterschiedlichem Erkenntnisinteresse dem Phänomen 'Glaubwürdigkeit' genähert haben und die daher jeweils unterschiedliche Signale in den Mittelpunkt ihrer Betrachtung stellen.

Ziel der verhaltensorientierten Glaubwürdigkeitsbeurteilung ist, bei einem Sprecher im Rahmen der direkten Kommunikation Hinweise auf Täuschungsversuche allein aus seinem Gebaren abzuleiten. Entsprechend wird in diesem Abschnitt der Arbeit dargestellt, welche Hinweise das die Kommunikation begleitende Verhalten liefert. Die forensische Aussagepsychologie beschäftigt sich mit der Glaubwürdigkeit von Zeugen; Indizien für die Aufrichtigkeit einer Person werden vorwiegend im Inhalt ihrer Aussage gesucht. Die quellen- und kontextorientierte Glaubwürdigkeitsforschung fragt nach verhaltensunabhängigen Merkmalen und Eigenschaften eines Sprechers, von denen seine Zuhörer auf seine Glaubwürdigkeit schließen, sowie nach der Bedeutung der Kommunikationssituation. Dieser Frage wird im Rahmen der Einstellungsforschung seit nunmehr über 50 Jahren nachgegangen. Da diese Forschungsbemühungen Aufschlüsse nicht nur für die direkte, sondern auch für die vermittelte Kommunikation liefern, wird der Darstellung der Befunde hier besondere Aufmerksamkeit zuteil.

Medien stellen eine spezielle Art von Informationsquellen dar. Untersuchungen, die sich mit ihrer Glaubwürdigkeit beschäftigen, werden daher in einem eigenen Kapitel gesondert vorgestellt. Daß die Frage nach der Glaubwürdigkeit von Medien nicht ausreicht, um das Problem der Glaubwürdigkeit im Kontext der Massenkommunikation zu erhellen, wird im Anschluß gezeigt. Auf der Grundlage des zeitungswissenschaftlichen Modells der Massenkommunikation wird die Notwendigkeit der Unterscheidung von Medien und Primärquellen erläutert und es wird dargelegt, welche Konsequenzen diese 
Trennung hat, insbesondere, welche Rolle die professionellen Vermittler bei der Zuschreibung von Glaubwürdigkeit spielen können.

Die theoretischen Einsichten werden schließlich anhand eines Fallbeispiels präzisiert: In einer Inhaltsanalyse mit Pilotcharakter wurde zum einen untersucht, welchen Stellenwert die Glaubwürdigkeit der beteiligten Akteure im speziellen Fall der Berichterstattung über politische Skandale hat, und zum anderen, ob und gegebenenfalls auf welche Weise die Vermittler daran teilhaben, daß einer Person Glaubwürdigkeit zu- oder abgesprochen wird.

Auf eine ausführliche Darstellung der inhaltsanalytischen Befunde wird an dieser Stelle aus jedoch verzichtet. ${ }^{1}$ Im Schlußabschnitt werden statt dessen die Erkenntnisse aus den vorangegangenen Kapiteln zusammengefaßt und im Hinblick auf ihre Brauchbarkeit für inhaltsanalytische Untersuchungen diskutiert. Dazu werden Überlegungen angestellt, welche der Glaubwürdigkeitsmerkmale aus den unterschiedlichen psychologischen Forschungsrichtungen auf welche Weise für eine inhaltsanalytische Vorgehensweise operationalisiert werden können. Die Ergebnisse der Pilotstudie schließlich illustrieren die Möglichkeiten der Umsetzung und geben Hinweise auf die Brauchbarkeit der gefundenen Kategorien.

Eine weitere Veröffentlichung, die den Schwerpunkt auf die empirischen Ergebnisse legt, ist in Vorbereitung. Interessierte sind vorerst herzlich dazu aufgefordert, die Dissertation im Original sowie das Kategorienschema bei der Verfasserin einzusehen. 


\section{Was ist Glaubwürdigkeit? Begriffsklärung und Definition}

Um sich in einer immer komplexer werdenden Umwelt zurechtzufinden, ist jeder von uns darauf angewiesen, Informationen über verschiedenste Sachverhalte zu erlangen, wobei er zu den meisten dieser Sachverhalte selbst keinen unmittelbaren Zugang hat. Daher muß er sich auf die Zuverlässigkeit dieser Informationen verlassen können: "Allgemein können wir sagen: Immer dann, wenn Informationen entscheidungs- oder handlungsrelevant werden, die uns nicht aus eigener Wahrnehmung bekannt sind, stellt sich prinzipiell die Frage nach deren Glaubwürdigkeit." ${ }^{2}$

Was aber ist unter Glaubwürdigkeit zu verstehen? Förmliche Definitionen sind dünn gesät; selbst wissenschaftliche Untersuchungen schreiten in der Regel gleich zur Operationalisierung, ohne eine exakte Begriffsklärung vorzunehmen. Dies deutet darauf hin, daß es sich um einen Alltagsbegriff handelt, der im Verständnis der meisten Leute keiner näheren Erklärung zu bedürfen scheint. Da allerdings die vorliegenden Definitionen vom Alltagsgebrauch durchaus abweichen, ist zunächst eine genauere Betrachtung des Begriffs angebracht:

"Im 15. Jahrhundert kommt es [das Wort glaubwürdig] zuerst in der Sprache des Rechtslebens auf und verdrängt allmählich das aus dem Mittelhochdeutschen stammende Wort 'gloubhaft' und bedeutet soviel wie vertrauenswürdig. Deshalb wird es zuerst in dem Sinne von Personen

2 Günter Köhnken: Glaubwürdigkeit: Untersuchungen zu einem psychologischen Konstrukt, München 1990, S. 1 (Hervorhebung auch im Original). 
gebraucht, daß man ihnen Vertrauen schenken kann hinsichtlich ihrer Wahrhaftigkeit und Zuverlässigkeit. Darüber hinaus wird es auch von Aussagen gebraucht, von Zeugnissen, Nachrichten, Berechnungen, weil sie der Wahrheit zu entsprechen scheinen, ohne daß objektive Beweise möglich wären." ${ }^{3}$

Glaubwürdigkeit kann sich dieser Einordnung gemäß sowohl auf Personen als auch auf deren Aussagen beziehen. Zusätzlich werden hier erste Anhaltspunkte für eine inhaltliche Präzisierung gegeben. Eine ähnliche Auffassung vertritt auch Bentele, der den kommunikativen Aspekt betont und konkrete Hinweise auf Personenmerkmale gibt:

"Glaubwürdigkeit läßt sich bestimmen als eine Eigenschaft, die Menschen, Institutionen oder deren kommunikativen Produkten (mündliche oder schriftliche Texte, audiovisuelle Darstellungen) zugeschrieben wird. Glaubwürdigkeit wird hier also nicht als inhärente Eigenschaft von Texten verstanden, sondern als Element einer zumindest vierstelligen Relation. Glaubwürdigkeit einer Person (oder Institution) X ist gegeben, wenn zumindest zwei Bedingungen erfüllt sind: a) der Kommunikationspartner (oder Rezipient) Y muß darauf vertrauen können, daß die Aussagen $\mathrm{x}_{1 \text {-n }}$ über die Ereignisse $\mathrm{z}_{1 \text {-n }}$ wahr sind, daß sie $\mathrm{z}_{1 \text {-n }}$ adäquat beschreiben; b) das kommunikative Verhalten von X muß ein Mindestmaß an Kohärenz aufweisen, es muß 'stimmig' sein. Dieses Vertrauen stellt sich durch oftmalige positive Erfahrungen her oder wird durch Merkmale wie gesellschaftlichen Status, Sachverständigkeit, Unabhängigkeit von Partialinteressen usw. konstituiert und unterstützt." ${ }^{4}$

Vereinzelt wird der Versuch unternommen, die Faktoren, die bereits Aristoteles einem glaubwürdigen Redner zugeschrieben hat, als Definitionsbestandteile zu übernehmen. Demnach umfaßt die Glaubwürdigkeit einer Person drei Komponenten: guten Charakter (die Ehrlichkeit des Redners), guten Willen (die 'Reinheit' oder Lauterkeit seiner Motive) und Weisheit, also seine

Wilhelm Gössmann: Glaubwürdigkeit im Sprachgebrauch. Stilkritische und sprachdidaktische Untersuchungen, München 1970, S. 23. Zur historischen und gegenwärtigen Verwendung des Begriffs vgl. auch: John Hennig: Sprache und Glaubwürdigkeit, in: Zeitschrift für deutsche Sprache 22/1966, S. 112-121.

4

Günter Bentele: Der Faktor Glaubwürdigkeit. Forschungsergebnisse und Fragen für die Sozialisationsperspektive, in: Publizistik 33/1988, S. 406-426, S. 408. 
Kompetenz. ${ }^{5}$

In ähnlicher Weise legen Hovland et al. die Glaubwürdigkeit des 'Kommunikators' (der eine beliebige Quelle sein kann) fest:

\begin{abstract}
"An individual's tendency to accept a conclusion advocated by a given communicator will depend in part upon how well informed and intelligent he believes the communicator to be. However, a recipient may believe that a communicator is capable of transmitting valid statements, but still be inclined to reject the communication if he suspects the communicator is motivated to make nonvalid assertions." 6
\end{abstract}

Diese Annahme führt zu den Dimensionen 'expertness' (Kompetenz) und 'trustworthiness' (Vertrauenswürdigkeit), wobei letztere sowohl die Ehrlichkeit als auch die Aufrichtigkeit der Motive umfaßt. Glaubwürdigkeit wird im folgenden als eine Funktion dieser beiden Dimensionen aufgefaßt: "In any given case, the weight given a communicator's assertions by his audience will depend upon both of these factors, and this resultant value can be referred to as the 'credibility' of the communicator."

Der Begriff Glaubwürdigkeit wird hier auf den Produzenten der Aussage, nicht aber auf die Aussage selbst angewendet, eine Einschränkung, die auch auf

5 Vgl. Dominic A. Infante / Andrew S. Rancer / Deanna F. Womack: Building Communication Theory, Illinois ${ }^{2} 1993$, S. 189 und Timothy J. Meyer: Media Credibility: The State of the Research, in: Public Telecommunications Review 2/1974, S. 48-52, S. 48.

In einer Übersetzung von Paul Gohlke aus dem Jahr 1959 lautet die einschlägige Passage: "Die Überzeugungskraft des Redners beruht auf dreierlei. Soviel Dinge nämlich gibt es außer den Beweisen noch, auf die wir vertrauen, nämlich Besonnenheit, Tugend und Wohlwollen. Denn man täuscht auch über den Gegenstand seiner Rede oder seines Rates durch alles dies oder eines davon. Entweder nämlich hat man aus Unverstand gar nicht erst die rechte Auffassung, oder man sagt aus Schlechtigkeit gar nicht, was man meint, oder man ist klug und billigdenkend, aber ohne Wohlwollen, so daß man womöglich trotz aller Einsicht nicht das Beste anrät. Weiter gibt es nichts. Folglich muß jemand, der alle diese Voraussetzungen erfüllt, überzeugend wirken." (Aristoteles: Rhetorik, Buch II, Kapitel 1, Paderborn 1959, S. 105.)

Ebd. 
die folgende Definition zutrifft:

"Glaubwürdigkeit liegt vor, wenn ein Kommunikator an einen Rezipienten eine Information vermittelt, von der er glaubt, daß sie zutreffend ist, wenn also der Kommunikator keine Täuschungsabsicht hat. Entscheidendes Bestimmungsstück ist hierbei die Intentionalität. Dies impliziert, daß der Kommunikator irrtümlich, d.h. ohne Vorsatz, eine falsche Information vermitteln kann, ohne unglaubwürdig zu sein. Ebenso kann er irrtümlich eine richtige Information vermitteln und dennoch unglaubwürdig sein, nämlich dann, wenn er die richtige Information für falsch hält." 8

Mit dieser Festlegung weicht Köhnken in einem wichtigen Punkt von den vorher genannten Definitionen ab: Während hier Glaubwürdigkeit ausschließlich an der Absicht des 'Kommunikators' festgemacht wird, vertreten die übrigen Autoren die Auffassung, daß sich Glaubwürdigkeit aus verschiedenen Komponenten zusammensetzt, die dem 'Kommunikator' von den Rezipienten zugeschrieben werden. ${ }^{9}$ Als entscheidend gilt also, was die Rezipienten wahrnehmen, nicht, was der 'Kommunikator' beabsichtigt.

Eine solche rezipientenorientierte Sicht liegt auch dieser Arbeit zugrunde. Es wird allerdings nicht darum gehen, Charakteristika zu suchen, welche die Rezipienten bei den 'Kommunikatoren' tatsächlich wahrnehmen. Vielmehr soll geprüft werden, welche Charakteristika den 'Kommunikatoren' in der vermittelten Kommunikation zugeschrieben werden - sei es von seiten anderer oder basierend auf eigenen Erklärungen. Daraus ergibt sich im Idealfall die Basis, die den Rezipienten von den Medien für ihre eigene Einschätzung geliefert wird.

Köhnken (1990), S. 4. (Hervorhebungen im Original.)

9

Ebenfalls explizit formuliert findet sich diese Einschätzung bei: Robert K. Tiemens: Some Relationships of Camera Angle to Communicator Credibility, in: Journal of Broadcasting 14/1970, S. 483-490, S. 484; Thomas A. Mc Cain / Joseph Chilberg / Jacob Wakshlag: The Effect of Camera Angle on Source Credibility and Attraction, in: Journal of Broadcasting 21/1977, S. 35-46, S. 37; Kenneth Starck: Media Credibility in Finland: A Cross-Cultural Approach, in: Journalism Quarterly 46/1969, S. 790-795, S. 790. Der Forschungsüberblick wird zeigen, daß diese Sichtweise in allen Studien zumindest implizit geteilt wird. 
Zu prüfen ist also im folgenden zweierlei: Zunächst soll hinterfragt werden, welcher Art die Zuschreibungen sind, die für Glaubwürdigkeit verantwortlich sind und auf welche Weise sie erfolgen. Dann muß geklärt werden, wer im Rahmen der Massenkommunikation solche Zuschreibungen abgibt, also wer mit dem Begriff 'Kommunikator' gemeint ist.

Die vorrangige Frage lautet also: Worauf gründet sich Glaubwürdigkeit? Um darüber Aufschluß zu erlangen, werden die Herangehensweisen unterschiedlicher Forschungsrichtungen dargestellt und geprüft. Der Einteilung von Köhnken folgend, der den wohl umfassendsten Überblick zur Erforschung der Glaubwürdigkeit liefert, ergeben sich aus psychologischer Sicht vier mögliche Fragestellungen:

1) Wie entwickelt sich das Verständnis und die moralische Bewertung von Lügen und Täuschungen sowie die Fähigkeit zur Täuschung und zum Erkennen der Täuschung?

2) Unter welchen Bedingungen neigt der Kommunikator zu glaubwürdigem oder unglaubwürdigem Verhalten?

3) Wie und mit welchem Erfolg beurteilen Rezipienten die Glaubwürdigkeit von Kommunikatoren und deren Äußerungen?

4) Welche Folgen haben Glaubwürdigkeitszuschreibungen für soziale Beziehungen? ${ }^{10}$

Für einen kommunikationswissenschaftlichen Zugang ist insbesondere die dritte dieser Fragestellungen von Belang. Entsprechend konzentriert sich die vorliegende Arbeit auf das Problem der Beurteilung von Glaubwürdigkeit, die wiederum zwei Perspektiven beinhaltet: Unter dem Stichwort Glaubwürdigkeitsdiagnostik wird untersucht, ob und wie zuverlässig "Täuschung und Wahrheit anhand bestimmter, mehr oder weniger objektiv zu erfassender 'Symptome' erkannt werden kann". ${ }^{11}$ Gefragt wird also nach den Verhaltenskorrelaten der Glaubwürdigkeit auf seiten des Kommunikators. Im zweiten Fall geht es darum zu prüfen, unter welchen Bedingungen die Zuschreibung von

$10 \quad$ Vgl. Köhnken (1990), S. 6.

11 Ebd. 
Glaubwürdigkeit zustande kommt, welche Mechanismen auf seiten der Rezipienten dafür verantwortlich sind und wie zutreffend derartige Zuschreibungen sein können. Im Blickpunkt der Aufmerksamkeit steht also die Glaubwürdigkeitsattribution. $^{12}$

Diesen Fragestellungen haben sich im wesentlichen drei unterschiedliche Forschungsbereiche angenommen, die jeweils andere Aspekte der Glaubwürdigkeitsbeurteilung in den Vordergrund stellen:

a) Die verhaltensorientierte Glaubwürdigkeitsbeurteilung "beschäftigt sich mit der Frage, ob die Glaubwürdigkeit einer Äußerung anhand bestimmter, die Kommunikation begleitender Verhaltensweisen erkannt werden kann"13, zielt also vorwiegend auf die Prüfung des nonverbalen Verhaltens und des Sprechverhaltens des 'Kommunikators'.

b) Im Rahmen der forensischen Aussagepsychologie steht die inhaltsorientierte Glaubwürdigkeitsbeurteilung im Mittelpunkt der Aufmerksamkeit. Hier wird gezielt nach Merkmalen gesucht, "die in wahren, aber nicht oder nur sehr selten in falschen Aussagen auftreten und somit eine ausreichend zuverlässige Beurteilung des Wahrheitsgehalts von Aussagen ermöglichen sollen." ${ }^{14}$

c) Die Einstellungs- und Persuasionsforschung schließlich konzentriert sich auf eine quellen- und kontextorientierte Glaubwürdigkeitsbeurteilung. Geprüft wird hier, "durch welche verhaltensunabhängigen Merkmale des Kommunikators, ggf. in Verbindung mit Aspekten des Kommunikationskontextes, Rezipienten zu dem Eindruck gelangen, daß eine Information glaubwürdig ist oder nicht." ${ }^{15}$

$\begin{array}{ll}12 & \text { Vgl. ebd. } \\ 13 & \text { Ebd. S. 9. } \\ 14 & \text { Ebd. S. 83. } \\ 15 & \text { Ebd. S. 7. }\end{array}$


Berührungspunkte mit kommunikationswissenschaftlichen Interessen ergeben sich in erster Linie mit dem letzten Forschungsbereich, dem daher das Hauptaugenmerk gilt. Dennoch sollen die wichtigsten Forschungsergebnisse aus dem verhaltens- und inhaltsorientierten Bereich daraufhin überprüft werden, ob sie brauchbare, inhaltsanalytisch operationalisierbare Kriterien enthalten, die für Glaubwürdigkeitszuschreibungen in Massenmedien relevant sind. 


\title{
3 Lügen haben kurze Beine? Die verhaltensorientierte Glaubwürdigkeitsbeurteilung
}

\author{
"Wieso wißt Ihr denn, daß ich eine Lüge gesagt habe?" \\ "Lügen, mein Kind, kann man gleich erkennen. Es gibt nämlich \\ zweierlei Arten: Da sind die Lügen, die kurze Beine haben, und \\ dann die Lügen, die lange Nasen haben. Deine Lügen gehörten \\ genau zu denen, die eine lange Nase haben." ${ }^{1}$
}

\subsection{Vorgehensweise und Befunde}

So einfach wie im Märchen ist es in der Realität nicht, Merkmale für Täuschungen auszumachen. Dennoch versucht eine psychologische Forschungsrichtung - die verhaltensorientierte Glaubwürdigkeitsbeurteilung -, Hinweise zu ermitteln und zu entschlüsseln, die ein Täuschungsverhalten nahelegen. Es geht also darum, "wahre und unwahre Äußerungen durch die Beobachtung von Phänomenen zu unterscheiden, die von dem Aussagenden nicht oder nur eingeschränkt kontrollierbar sind". ${ }^{2}$ Dahinter steht die Vorstellung, daß bewußte Täuschungen mit psychischen Prozessen verknüpft sind, die sich im beobachtbaren Verhalten des Lügners niederschlagen. ${ }^{3}$ Systematische Forschun-

1 Carlo Collodi: Das Märchen vom Pinoccio, Zürich 1968, S. 50.

2 Köhnken (1990), S. 12.

3

$\mathrm{Zu}$ den auslösenden psychischen Prozessen gehört etwa verstärkte Erregung (wegen der Furcht, durchschaut oder bestraft zu werden) und das Bemühen, diese zu kontrollieren. Vgl. Michael J. Cody / Peter J. Marston / Myrna Foster: Deception: Paralinguistic and Verbal Leakage, in: Robert N. Bostrom / Bruce H. Westley (Hg.): Communication Yearbook 8, Beverly Hills / London / New Delhi 1984, 464-490, S. 466f. sowie Miron Zuckerman / Bella M. DePaulo / Robert Rosenthal: Verbal and Nonverbal Communication of Deception, in: Leonard 
gen gibt es erst seit den 40er Jahren; die Forschungsbemühungen verstärkten sich seit den späten 60er Jahren, einhergehend mit dem zunehmenden Interesse an nonverbaler Kommunikation. ${ }^{4}$

Unterschieden wird zwischen kommunikator- und rezipientenorientierter Perspektive. Aus der kommunikatororientierten Perspektive ist danach zu fragen, welche beobachtbaren Verhaltensweisen als Begleiterscheinungen von Täuschungen auftreten, unter welchen Bedingungen von den Hinweisreizen auf die Glaubwürdigkeit der Informationen geschlossen werden kann und inwieweit die auftretenden Verhaltensweisen exklusiv auf Täuschungen beschränkt sind. Folgt man der rezipientenorientierten Perspektive, ist zu ergründen, welche der auftretenden Reize überhaupt von Beobachtern wahrgenommen werden und welche dieser Wahrnehmungen Schlußfolgerungen auf Täuschungen nach sich ziehen. Damit verknüpft ist die Frage, wie korrekt solche Einschätzungen sind, wie gut Rezipienten wahre und falsche Äußerungen unterscheiden können. Es geht also auf Kommunikatorseite um Ausdrucksprozesse und auf Rezipientenseite um Eindrucks- oder Urteilsprozesse. ${ }^{5}$

In experimentellen Versuchsanordnungen werden häufig beide Ansätze

Berkowitz (Hrsg.): Advances in Experimental Social Psychology, Vol. 14, New York/London 1981, S. 1-59.

$4 \quad$ Vgl. Köhnken (1990), S. 12f. Zur nonverbalen Kommunikation siehe den Überblick von Judee K. Burgoon: Nonverbal Communication Research in the 1970s: An Overview, in: Dan Nimmo (Hrsg.): Communication Yearbook 4, New Brunswick / London 1980, S. 179-197. Zum Täuschungsverhalten siehe etwa: Mark L. Knapp / Mark E. Comadena: Telling It Like It Isn't: A Review of Theory and Research on Deceptive Communications, in: Human Communication Research 5/1979, S. 270-285; Norman R. F. Maier: Sensivity to Attempts at Deception in an Interview, in: Personnel Psychology 19/1965, S. 55-66; Norman R. F. Maier / James A. Thurber: Accuracy of Judgments of Deception When an Interview is Watched, Heard, and Read, in: Personnel Psychology 21/1968, S. 23-30; John E. Hocking et al.: Detecting Deceptive Communication from Verbal, Visual, and Paralinguistic Cues, in: Human Communication Research 6/1979, S. 33-46; Paul Ekman / Wallace V. Friesen: Nonverbal Leakage and Clues to Deception, in: Psychatry 32/1969, S. 88-105. Eine Zusammenfassung der wichtigsten Ergebnisse liefert Paul Ekman: Weshalb Lügen kurze Beine haben. Über Täuschungen und deren Aufdeckung im privaten und öffentlichen Leben, Berlin/New York 1989.

Vgl. Köhnken (1990), S. 18ff. 
verknüpft. Einerseits werden Akteure gebeten, zu bestimmten Sachverhalten jeweils wahre und falsche Antworten zu geben. Das Antwortverhalten wird mittels Videoaufzeichnung festgehalten und Versuchspersonen gezeigt, worauf diese ihre Glaubwürdigkeitseinschätzungen abgeben. ${ }^{6}$ Diese Vorgehensweise bringt es mit sich, daß nicht einzelne Indikatoren isoliert betrachtet oder kontrolliert werden, sondern die Schlußfolgerungen auf dem gesamten sichtbaren Verhalten basieren. Obgleich also einzelne Elemente für sich genommen nur bedingt aussagekräftig sind, werden nachfolgend die Faktoren, die für die Glaubwürdigkeitsbeurteilung herangezogen werden, getrennt vorgestellt und betrachtet, um einen ersten Überblick zu gewährleisten.?

Eine Reihe von relevanten Indikatoren liefert das nonverbale Verhalten. Betrachtet wird hier ausschließlich die "Körpersprache". Zu den Verhaltenselementen im Kopfbereich gehört neben Veränderungen des Pupillendurchmessers, die mittels einer Apparatur gemessen werden, die Lidschlagfrequenz. Bislang blieb jedoch offen, ob überdurchschnittlich häufiges oder eher seltenes reflexhaftes Blinzeln den Lügner kennzeichnet. ${ }^{8}$ Weniger umstritten sind ren) soll hier verzichtet werden. Vgl. dazu im Überblick: D. T. Lykken: Psychology and the Lie Detector Industry, in: American Psychologist 29/1974, S. 725-739.

Die Gliederung erfolgt in Anlehnung an die zusammenfassende Schautafel bei Köhnken (1990), S. 10f.

Belege lassen sich für beide Varianten finden: Kraut ist nach einer Sichtung von 21 Studien, von denen sich fünf mit der Lidschlagfrequenz befassen, der Auffassung, daß häufiger Lidschlag eines der aussagekräftigsten Kriterien für Täuschungen ist (vgl. Robert Kraut: Humans as Lie Detectors. Some Second Thoughts, in: Journal of Communication 30/1980, S. 209-216, S. 212); eine der von ihm benannten Untersuchungen besagt allerdings das Gegenteil (vgl. R. J. Cutrow et. al.: The Objective Use of Multiple Physiological Indices in the Detection of Deception, in: Psychophysiology 9/1972, S. 578-588). Eine reduzierte Lidschlagfrequenz im Fall von Täuschungen ermitteln auch John E. Hocking / D. G. Leathers: Nonverbal Indicators of Deception: A New Theoretical Perspective, in: Communication Monographs 47/1980, S. 119131. Aus diesen widersprüchlichen Befunden könnte man die Vermutung ableiten, daß sich die Lidschlagfrequenz von Nichtlügnern in einem gewissen Intervall bewegt und daß Abweichungen von dieser Norm in beide Richtungen (seltener oder häufiger) auf Lügen hindeuten können. 
Häufigkeit und/oder Dauer des Blickkontakts mit dem Gesprächspartner: Lügner weichen den Blicken ihrer Gesprächspartner häufiger aus. ${ }^{9}$ Lächeln, das zu den bevorzugten Kriterien auf seiten der Rezipienten gehört, wird wiederum unterschiedlich interpretiert. ${ }^{10}$ Einbezogen werden noch weitere Elemente der Mimik, etwa Mundbewegungen sowie Kopfbewegungen, insbesondere Häufigkeit und Intensität von Kopfschütteln oder Niken. ${ }^{11}$

Ekman/Friesen kamen zu dem Ergebnis, daß Akteure bei Täuschungsversuchen vor allem danach trachten, Bewegungen im Gesichtsbereich von vornherein zu kontrollieren. Dies geschieht, indem sie Verhaltensweisen gezielt einsetzen oder unterdrücken. Dagegen wird den Körperbewegungen weniger Aufmerksamkeit geschenkt. Letztere liefern mithin eher Hinweisreize für lügenhaftes Verhalten. ${ }^{12}$ Entsprechend könnte eine mögliche Erklärung für die

9 Vgl. z.B. J. A. Burns / B. L. Kintz: Eye Contact While Lying During an Interview, in: Bulletin of the Psychonomic Society 2/1976, S. 87-89 und Mark L. Knapp / R. P. Hart / H. S. Dennis: An Exploration of Deception as a Communication Construct, in: Human Communication Research 1/1974, S. 15-29.

10 Vgl. Robert E. Kraut: Verbal and Nonverbal Cues in the Perception of Lying, in: Journal of Personality and Social Psychology 36/1978, S. 380-391. Hier wird berichtet, daß Akteure die weniger lächeln, als aufrichtiger eingestuft werden; hingegen stuft Köhnkens Zusammenfassung (1990: 54) seltenes Lächeln als Indiz für Unwahrheit ein. Auch hier besteht, wie bei der Lidschlagfrequenz, also die Möglichkeit, daß eine Abweichung vom normalen Verhalten in beide Richtungen Hinweise für Täuschungen liefert. Plausibel erscheint auch, daß die Häufigkeit des Lächelns für sich genommen nicht allzu aussagekräftig ist. Zusätzlich ist die Qualität des Lächelns in Betracht zu ziehen. Ekman (1989: 122ff.) listet nicht weniger als achtzehn verschiedene Arten des Lächelns auf und führt aus, daß in Täuschungssituationen häufiger ein "falsches" Lächeln zu beobachten ist, das entweder eine positive Emotion vortäuscht oder als Maske zur Verheimlichung negativer Emotionen dient.

Solche mimischen Mittel werden besonders differenziert bei: Robert S. Feldman / Linda Devin-Sheenan / Vernon L. Allen: Nonverbal Cues as Indicators of Verbal Dissembling, in: American Educational Research Journal 15/1978, S. 217-231. Sie erheben u.a. Stirnrunzeln, Heben der Augenbrauen, Schürzen der sowie Beißen auf die Lippen sowie Mundhaltungen, die Mißmut ausdrücken.

Vgl. Paul Ekman / Wallace V. Friesen: Detecting Deception from the Body or Face, in: Journal of Personality and Social Psychology 29/1974, S. 288-298. Siehe dazu auch Paul Ekman et al.: Relative Importance of Face, Body and Speech in Judgments of Personality and Affect, in: Journal of Personality and Social Psychology 38/1980, S. 270-277; Hocking et al. 
Uneinheitlichkeit der Befunde darin liegen, daß in den Studien unterschiedlich talentierte "Lügner" auftreten oder daß individuell unterschiedliche Strategien zur Verschleierung des eigenen Verhaltens verwendet werden. So kann etwa ein Akteur der Auffassung sein, daß er mit häufigem Lächeln den Eindruck von Aufrichtigkeit erzeugt, während ein anderer den gleichen Eindruck mittels eines ernsten Gesichtsausdrucks vermitteln will.

Verhaltenselemente im Körperbereich, von denen man sich Rückschlüsse auf den Wahrheitsgehalt einer Aussage verspricht, sind im oberen Körperbereich Achselzucken und Gestik, die sogenannten Illustratoren. Darunter versteht man "Arm- und Handbewegungen, die das jeweils Gesagte ergänzen oder modifizieren". ${ }^{13}$ Besondere Bedeutung kommt sogenannten Adaptoren zu, also Selbstmanipulationen wie Kratzen oder Zupfen an den Haaren oder Objektmanipulationen wie Spielen mit dem Schreibgerät. Im Unterschied zur Gestik haben solche Handlungen keine illustrierende Funktion und somit keinen Bezug zum Inhalt der Äußerung. ${ }^{14}$ Im Fall von Täuschungen treten solche Adaptoren häufiger auf, während sich die Zahl der Illustratoren verringert. ${ }^{15}$ Bein- und Fußbewegungen ${ }^{16}$ werden schließlich ebenso erfaßt wie die Veränderungen der Körperhaltung.

Große Aufmerksamkeit erfährt auch das extralinguistische Verhalten.

(1979); G. E. Littlepage / M. A. Pineault: Verbal, Facial and Paralinguistic Cues to the Detection of Truth and Lying, in: Personality and Social Psychology Bulletin 4/1978, S. 461464; dies.: Detection of Deceptive Factual Statements from the Body and the Face, in: Personality and Social Psychology Bulletin 5/1979, S. 325-328. in: Journal of Communication 22/1972, S. 353-374. Indicators of Affect and Deception in an Interview Setting, in: Journal of Applied Social Psychology 5/1975, S. 54-67. 
Darunter versteht man alle sprachlichen Eigenheiten, die keine inhaltliche Bedeutung haben. Dazu gehört laut Köhnken die Reaktionslatenz, also die Zeitdauer zwischen dem Ende einer Frage und dem Beginn der Antwort, die Antwortlänge, die Sprechgeschwindigkeit, Sprechstörungen (Stottern und Versprecher ebenso wie grammatikalische Fehler oder unvollständige Sätze), Verzögerungen im Verlauf der Antwort (stille Pausen oder stimmhafte Pausen, die mit 'äh' o.ä. gefüllt werden) sowie die Stimmhöhe. ${ }^{17}$ An anderer Stelle findet sich noch die Stimmqualität als Indikator. ${ }^{18}$

Anders als im Bereich der forensischen Glaubwürdigkeitsbeurteilung legt die verhaltensorientierte Forschungsrichtung wenig Wert auf Merkmale des Inhalts der Aussage. In Betracht gezogen wird die Anzahl der negativen Äußerungen, die Erwähnung irrelevanter Details, die Anzahl der Selbstreferenzen (die Verwendung von 'ich' oder 'mein'), die Unmittelbarkeit (Äußerungen, die auf die Enge oder Intimität einer Interaktion verweisen) sowie Übergeneralisierungen (die Häufigkeit der Verwendung von Wörtern wie 'immer', 'alle' etc.). ${ }^{19}$ Bei unvorbereiteten Lügen ist zudem die Länge der Mitteilung bedeutsam. ${ }^{20}$ Weiteren Aufschluß über Täuschungen geben schließlich Kanaldiskrepanzen, also unterschiedliche oder widersprüchliche

17 Vgl. Köhnken (1990), S. 11 und Miron Zuckerman et al.: Facial and Vocal Cues of Deception and Honesty, in: Journal of Experimental Social Psychology 15/1979, S. 378-396. Zur Stimmhöhe siehe auch Paul Ekman / Wallace V. Friesen / Klaus Scherer: Body Movement and Voice Pitch in Deceptive Interaction, in: Semiotica 16/1976, S. 23-27. Zu Sprechstörungen vgl. A. Mehrabian: Nonverbal Betraying of Feeling, in: Journal of Experimental Research in Personality 5/1975, S. 64-73. Zu Reaktionslatenz und Antwortlänge siehe Michael J. Cody / Peter J. Marston / Myrna Foster: Deception: Paralinguistic and Verbal Leakage, in: Robert N. Bostrom / Bruce H. Westley (Hg.): Communication Yearbook 8, Beverly Hills / London / New Delhi 1984, 464-490.

18 Vgl. David W. Addington: The Effect of Vocal Variations on Ratings of Source Credibility, in: Speech Monographs 38/1971, S. 242-247. Hier wird gezeigt, daß Heiserkeit den Eindruck der Glaubwürdigkeit negativ beeinflußt.

19 Vgl. Köhnken (1990), S. 11.

20 Vgl. Cody / Marston / Foster (1984), S. 485f. 
Verhaltensweisen in den verschiedenen - verbalen, nonverbalen oder extralinguistischen - Kommunikationskanälen. ${ }^{21}$

Der Nachweis, daß Täuschungsversuche von verschiedenen beobachtbaren Verhaltensweisen begleitet werden, kann aufgrund der Vielzahl der Studien, die solches feststellen, als gesichert gelten:

"Im Gesamtdurchschnitt der vorliegenden experimentellen Studien handelt es sich hierbei um verstärkte Pupillendilatationen, erhöhte Lidschlagfrequenz, vermehrtes Auftreten von Adaptoren sowie eine Zunahme von Sprechfehlern und Verzögerungsphänomenen im Sprechfluß. Ferner steigt die Tonhöhe an, die Antworten werden kürzer und enthalten mehr negative, irrelevante und distanzierende Äußerungen." 22

Festzuhalten bleibt allerdings, daß es "das universelle 'Wahrheits- oder Lügensignal' im Sinne eines Verhaltensmusters, das konsistent, d.h. personenund situationsübergreifend die Klassifizierung einer Darstellung als 'wahr' oder 'gelogen' erlauben würde", nicht gibt. ${ }^{23}$ Neben persönlichen, individuellen Merkmalen spielen bei der Fähigkeit, erfolgreich zu täuschen, offenbar auch die Variablen Alter und Geschlecht eine Rolle. Je älter Kinder werden, desto besser gelingt es ihnen, Unwahrheiten zu bemänteln. ${ }^{24}$ Ferner gibt es Hinweise, daß Frauen im Sozialisationsverlauf besser als Männer gelernt haben, ihr nonverbales Verhalten, insbesondere die Mimik, zu beherrschen. ${ }^{25}$

\footnotetext{
$21 \quad$ Vgl. Köhnken (1990), S. 11.

22 Köhnken (1990), S. 43.

23 Köhnken (1990), S. 46.

24 Vgl. Robert S. Feldman / John B. White: Detecting Deception in Children, in: Journal of Communication 30/1980, S. 121-128 und Robert S. Feldman / S. L. Jenkins / O. Popoola: Detection of Deception in Adults and Children via Facial Expressions, in: Child Development 49/1979, S. 1107-1113.

Vgl. Köhnken (1990), S. 45. Ferner: Michael J. Cody / H. D. O'Hair: Nonverbal Communication and Deception: Differences in Deception Cues Due to Gender and Communicator Dominance, in: Communication Monographs 50/1983, S.175-192.
} 
"Zusätzliche Variabilität entsteht durch den situativen Kontext der Kommunikation. Insbesondere die Stärke der Motivation des Kommunikators zur Vermeidung einer Entdeckung sowie die Möglichkeit, die Darstellung planen und vorbereiten zu können, manifestiert sich in differentiellen Verhaltensmustern. Ob auch der Täuschungsgegenstand und die Täuschungsform einen Einfluß auf distale Indikatorreize haben, ist bislang nicht völlig klar. Einige Indizien deuten jedoch darauf hin." 26

Die zum Teil widersprüchlichen Befunde können damit erklärt werden, daß die Kommunikationssituation und die Motivation der Quelle nicht genügend berücksichtigt wurden. Ekman kritisiert insbesondere, daß in fast allen Untersuchungen für die Versuchspersonen wenig auf dem Spiel stand und daß die Lügen selbst nur triviale Dinge betrafen. Ferner wird in der Regel die Beziehung zum Zielobjekt (der Person, die belogen wird) völlig außer acht gelassen. $^{27}$

Zudem ist zu berücksichtigen, daß die ermittelten Indikatoren keineswegs eindeutige Hinweise für Lügen sind, obgleich sie in den durchgeführten Experimenten häufig so eingestuft werden. Vielmehr verweisen sie zunächst auf das Vorhandensein von Emotionen verschiedener Art. Diese Emotionen können auftreten, weil der Sprecher lügt; sie können aber auch andere Ursachen haben. So ist

"ein Anstieg der Stimmhöhe ... kein Anzeichen für eine Täuschung, vielmehr ein Anzeichen für Angst oder Wut, vielleicht auch für Erregung. (...) Es besteht die Gefahr, die vokalen Anzeichen einer Emotion als Beweis für eine Täuschung zu interpretieren. Bei einer aufrichtigen Person, die sich Sorgen macht, daß man ihr keinen Glauben schenkt, kann aus dieser Angst heraus die Stimmhöhe in derselben Weise ansteigen, wie bei einer Lügnerin, die Angst davor hat, entdeckt zu werden. Das Problem für denjenigen, der eine Lüge entdecken will, besteht darin, daß nicht nur Lügner, sondern auch Unschuldige zuweilen emotional erregt sind." ${ }^{28}$

26 Köhnken (1990), S. 43.

$27 \quad$ Vgl. Ekman (1989), S. 68.

28 Ekman (1989), S. 71. Das gilt auch für andere Hinweisreize. So sind vermehrte Selbst- 
Da es im Rahmen dieser Arbeit darum geht, Glaubwürdigkeitszuschreibungen zu ermitteln, die für Rezipienten bedeutsam sind, sollen die Probleme, die aus psychologischer Sicht mit der Ermittlung tatsächlicher Glaubwürdigkeitsmerkmale verknüpft sind, hier nicht weiter vertieft werden. Interessanter ist die Frage, welche Vorstellungen auf Rezipientenseite hinsichtlich des Verhaltens von Lügnern bestehen. ${ }^{29}$ Die Befragungen zeigen übereinstimmend, daß ein festgefügtes Bild existiert:

"Danach sind Lügen in der Vorstellung der Probanden inhaltlich vor allem durch Beeinträchtigungen der Logik und Stimmigkeit sowie der Plausibilität gekennzeichnet. Ferner nehmen Widersprüche in der Darstellung zu und der Kommunikator vermeidet es, seine vermeintliche Glaubwürdigkeit durch für ihn selbst ungünstige Details oder durch eigene Zweifel an der Richtigkeit seiner Darstellung herabzusetzen. Sein Sprechverhalten ist gekennzeichnet durch verzögerte, ausweichende Antworten, die zudem mit vielen Floskeln und überflüssigen Wiederholungen durchsetzt sind. Zudem stottert er, räuspert sich oft und

und Objektmanipulationen zwar ein Anzeichen dafür, daß ein Sprecher nervös ist oder sich unbehaglich fühlt. Der Grund für sein Unbehagen muß aber nicht eine Lüge sein. Je nach Situation können Adaptoren aber auch ein Hinweis dafür sein, daß der Sprecher besonders entspannt ist. (Vgl. ebd. S. 87.) Für den sparsamen Einsatz von Gesten kann es ebenfalls mehrere Gründe geben: "Der erste Grund ist, daß bei dem, was gesagt wird, die emotionale Beteiligung fehlt. Man illustriert weniger als gewöhnlich, wenn man unbeteiligt, gelangweilt, desinteressiert oder tief betrübt ist. (...) Die Zahl der Illustratoren verringert sich auch dann, wenn jemand Schwierigkeiten hat, zu entscheiden, was genau er sagen soll. Wenn jemand jedes Wort sorgfältig abwägt und über das Gesagte, ehe es ausgesprochen wird, nachdenkt, wird er seine Äußerungen nicht mit vielen Illustratoren begleiten. Wenn jemand zum ersten- oder zweitenmal eine Rede hält, sei es eine Vorlesung oder einen Werbevortrag, wird er weniger Illustratoren verwenden als zu einem späteren Zeitpunkt, wenn es ihn weniger Mühe kostet, die richtigen Worte zu finden. Die Zahl der Illustratoren nimmt immer dann ab, wenn man beim Sprechen mit Vorsicht zu Werk geht. Das muß nichts mit Täuschung zu tun haben." (Ebd. S. 82f.)

Leider erfreut sich diese Frage nicht allzu großen Interesses. Neben Hocking / Leathers (1980) und Miron Zuckerman / R. Koestner / R. Driver: Beliefs about Cues Associated with Deception, in: Journal of Nonverbal Behavior 6/1981, S. 105-114 hat sich lediglich Köhnken in seiner unveröffentlichten Habilitationsschrift mit diesem Problem auseinandergesetzt. Für die Betrachtung wird daher auf dessen Zusammenfassung in Köhnken (1990), S. 47-62 zurückgegriffen. 
verspricht sich häufiger. Im Kopfbereich macht sich Nervosität bemerkbar, insbesondere durch Erröten, häufiges Schlucken, wechselhafte Mimik und einen unsteten Blick. Auch der übrige Körper vermittelt einen nervösen Gesamteindruck, der vor allem durch häufige Armbewegungen und Hantierungen am eigenen Körper oder mit irgendwelchen Gegenständen gekennzeichnet ist." ${ }^{30}$

Mit Ausnahme des Blickkontakts, von dem die Befragten glauben, daß er sich im Täuschungsfall verringert, wird davon ausgegangen, daß eine Zunahme der Häufigkeiten der genannten Verhaltensweisen charakteristisch für Lügner ist. ${ }^{31}$ Dabei erwarten die Befragten bei anderen Personen jeweils stärkere Zunahmen als bei sich selbst, das heißt sie sind der Auffassung, sie selbst könnten ihre Verhaltensweisen effektiver kontrollieren. ${ }^{32}$

Ferner zeigt sich, daß die vermuteten Verhaltensweisen von den tatsächlich ermittelten in erheblichem Maße abweichen. Es werden einerseits Indikatoren genannt, die in den durchgeführten Untersuchungen keine Rolle spielten, andererseits hat sich ergeben, daß zahlreiche Verhaltensweisen bei Täuschungen tendenziell seltener auftraten. ${ }^{33}$ Bei der Frage nach potentiellen Mißtrauensquellen wurden, nach der Nennung inhaltlicher Merkmale, Elemente der Mimik als am wichtigsten erachtet, gefolgt von Sprechverhalten und Körperbewegungen. Die tatsächlichen "Trefferquoten" belegen, daß hier wohl bei den meisten Menschen Fehleinschätzungen vorliegen. Demnach werden Täuschungen nämlich am besten anhand des Sprechverhaltens und der Inhalte durchschaut, auch die Elemente aus dem Körperbereich spielen noch eine gewisse Rolle. Was die Mimik anbelangt, so ist sie der mit Abstand schlechteste Indikator; die Anzahl der korrekt erkannten Täuschungen über-

\begin{tabular}{ll}
\hline 30 & Köhnken (1990), S. 49f. \\
31 & Vgl. Köhnken (1990), S. 50. \\
32 & Vgl. Köhnken (1990), S. 48. \\
33 & Vgl. Köhnken (1990), S. 52.
\end{tabular}


schreitet kaum den Zufallsbereich. ${ }^{34}$

Daraus läßt sich folgern, daß Menschen sich offenbar im allgemeinen als wenig brauchbare "Lügendetektoren" erweisen. Literaturüberblicke ergeben, daß die berichteten Werte für erkannte Täuschungen selten über 65 Prozent liegen, ${ }^{35}$ obwohl die meisten Personen ihre diesbezügliche Kompetenz durchaus hoch einschätzen. ${ }^{36}$ Selbst Beobachter, denen man von Berufs wegen eine höhere Kompetenz unterstellt, wie etwa Polizeibeamte, liefern keine zuverlässigeren Einschätzungen. ${ }^{37}$ Eine gewisse Vertrautheit mit dem "Lügner" - die in den meisten Versuchen dadurch erreicht wird, daß er zunächst in mehreren Sprechsituationen gezeigt wird, in denen er die Wahrheit sagt - oder

34 Vgl. Köhnken (1990), S. 60ff.

35

Vgl. Kraut (1980), S. 209; Mark E. Comadena: Accuracy in Detecting Deception: Intimate and Friendship Relations, in: Michael Burgoon / Noel Doran (Hg.): Communication Yearbook 6, Beverly Hills / London / New Delhi 1982, S. 446-472, S. 446. Zur Zuverlässigkeit der Einschätzungen siehe ferner: Norman R. F. Maier / J. Janzen: The Reliability of Persons Making Judgments of Honesty and Dishonesty, in: Perceptual and Motor Skills, 25/1967, 141151; Norman R. F. Maier / James A. Thurber: Accuracy of Judgments of Deception when an Interview is Watched, Heard and Read, in: Personnel Psychology 21/1968, S. 23-30; R. A. Maier / P. J. Lavrakas: Lying Behavior and Evaluation of Lies, in: Perceptual and Motor Skills 42/1976, S. 575-581. Zu individuellen Unterschieden bei "guten" und "schlechten" Dekodern nonverbaler Verhaltensweisen siehe David B. Buller / Judee K. Burgoon: The Effects of Vocalics and Nonverbal Sensitivity on Compliance. A Replication and Extension, in: Human Communication Research 13/1986, S. 126-144. Die Zuverlässigkeit der Einschätzungen ist nicht abhängig von der Menge der Information, die den Beobachtern zugänglich ist: direkte Beobachtung (durch eine Einwegscheibe), indirekte Beobachtung (mittels einer Videoaufzeichnung) oder die Beschränkung auf einzelne Übertragungskanäle (Audioaufnahme oder Transskript) führt - zumindest bei ungeübten Beobachtern - nicht zu signifikant unterschiedlichen Trefferquoten. (Vgl. Joyce E. Bauchner / David R. Brandt / Gerald R. Miller: The Truth/Deception Attribution: Effects of Varying Levels of Information Availability, in: Brent D. Ruben (Hrsg.): Communication Yearbook I, New Brunswick, N.J. 1977, S. 229-243.

Vgl. Köhnken (1990), S. 56f. Die Gründe für die niedrigen Trefferquoten sollen hier nicht näher betrachtet werden, da sie für die Ziele dieser Untersuchung nicht von Interesse sind. Zur Vertiefung siehe die Erklärversuche bei Kraut (1980) und Köhnken (1990), S. 56-74.

Vgl. Aldert Vrij: Credibility Judgments of Detectives: The Impact of Nonverbal Behavior, Social Sills, and Physical Characteristics on Impression Formation, in: Journal of Social Psychology 133/1993, S. 601-610. Lediglich in 49 Prozent der Fälle wurden Täuschungen korrekt als solche identifiziert. 
nähere Bekanntschaft steigert das Erkennen von Täuschungen. ${ }^{38}$ Ferner gibt es Anhaltspunkte dafür, daß Frauen Täuschungen besser erkennen. ${ }^{39}$

\subsection{Zwischenbilanz}

Im Rahmen der verhaltensorientierten Glaubwürdigkeitsforschung soll ermittelt werden, ob und inwieweit sich im Verhalten eines Kommunikators Indizien finden lassen, die es ermöglichen, wahre von falschen Aussagen zu trennen. Die Forschung konzentriert sich dabei auf das nonverbale Verhalten (Veränderungen des Pupillendurchmessers, Lidschlagfrequenz, Blickkontakt, Mimik, Gestik, Kopf- und Körperbewegungen) und das extralinguistische Verhalten (Antwortlänge, Sprechgeschwindigkeit, Reaktionslatenz, Verzögerungen, Sprechstörungen, Stimmhöhe, Stimmqualität).

Obgleich es kein universales Verhaltensmuster gibt, das einen schlüssigen Beweis für Lügen darstellt, kann mit einiger Sicherheit davon ausgegangen werden, daß bestimmte Verhaltensweisen bei unwahren Aussagen häufiger (Lidschlagfrequenz, Körperbewegungen, Reaktionslatenzen, Verzögerungen, Sprechstörungen) oder weniger häufig (Blickkontakt) auftreten. Allerdings müssen zur Beurteilung zusätzlich die Person und Persönlichkeit des Sprechers

38 Vgl. Comadena (1982), S. 466. Steven A. McCornack / Malcolm R. Parks: Deception Detection and Relationship Development and Relationship Development: The Other Side of Trust, in: Margaret L. McLaughlin (Hrsg.): Communication Yearbook 9, Beverly Hills /London / New Delhi 1986, S. 377-389; D. R. Brandt / Gerald R. Miller / John E. Hocking: The TruthDeception Attribution: Effects of Familiarity on the Ability of Observers to Detect Deception, in: Human Communication Research 6/1980, S. 99-110; Gerald R. Miller et al.: "...and nothing but the truth": How well can Observers Detect Deceptive Testimony? In: B. D. Sales (Hrsg.): Perspectives in Law and Psychology, Vol. II, The Jury, Judicial and Trial Process, New York 1981, S. 145-179.

39 Vgl. Comadena (1982), S. 466 und J. A. Hall: Gender Effects in Decoding Nonverbal Cues, in: Psychological Bulletin 3/1977, S. 446-449. Nicht bestätigt wird diese "weibliche Überlegenheit" bei Robert Rosenthal / Bella M. DePaulo: Sex Differences in Eavesdropping on Nonverbal Cues, in: Journal of Personality and Social Psychology 37/1979, S. 273-285. 
sowie die Kommunikationssituation berücksichtigt werden.

Neben den Ausdrucksprozessen auf seiten des Kommunikators werden Eindrucks- und Urteilsprozesse auf Rezipientenseite betrachtet. Dabei zeigt sich, daß Rezipienten ein festgefügtes Bild bezüglich des Verhaltens eines Lügners haben, welches sich mit den tatsächlichen Verhaltensweisen nur in Teilbereichen deckt. Entsprechend ist die Fähigkeit, unwahre Aussagen zu erkennen, insbesondere bei untrainierten Beobachtern, die den Kommunikator nicht kennen, nur schwach ausgeprägt. Inhaltliche Merkmale der Aussage (z.B. Logik, Plausibilität), die für Rezipienten eine große Rolle spielen, werden von der verhaltensorientierten Glaubwürdigkeitsforschung nur selten berücksichtigt.

Die Forschung liefert keinerlei Hinweise dafür, ob die ermittelten Hinweisreize auch als Indizien für Glaubwürdigkeit betrachtet werden, wenn sie den Rezipienten lediglich indirekt, etwa im Rahmen von Schilderungen in Printmedien, zugänglich sind. Da aber die Befragungen von Rezipienten so übereinstimmende Vorstellungen erbrachten, daß man "auf ein offenbar weitverbreitetes, markantes Stereotyp des 'typischen' Lügners schließen" kann, ${ }^{40}$ erscheint plausibel, daß solche Merkmale, auch wenn sie in Charakterisierungen auftreten, dieses Stereotyp aktivieren. Unabhängig davon müssen Verhaltensbeschreibungen, die als Indizien für wahre oder unwahre Äußerungen gelten können, berücksichtigt werden, wenn es darum geht, die Frage zu beantworten, ob und inwieweit Personen, als glaubwürdig oder unglaubwürdig dargestellt werden. Daher werden solche Verhaltensbeschreibungen, soweit sie befriedigend operationalisiert werden können, Bestandteil einer Inhaltsanalyse sein müssen. Zu klären ist dann auch, welche Rolle die Schilderung des Verhaltens des Kommunikators im Rahmen der Berichterstattung in Printmedien spielt und durch welche weiteren Faktoren solche Schilderungen ergänzt werden.

$40 \quad$ Köhnken (1990), S. 52. 


\section{Wer einmal lügt ... \\ Die inhaltsorientierte Glaubwürdigkeitsbeurteilung}

Die forensische Aussagepsychologie hat ihre Wurzeln im ausgehenden 19. Jahrhundert und gilt somit als "eines der ältesten Gebiete der angewandten Experimentalpsychologie überhaupt". ${ }^{41}$ Ziel der Forschung ist, Kriterien zu finden, die es ermöglichen, wahre und verfälschte Zeugenaussagen zu unterscheiden.

Ursprünglich wurde dabei postuliert, daß es ein relativ stabiles Persönlichkeitsmerkmal gäbe, daß sich mit 'Wahrheitsliebe' oder 'Wahrhaftigkeit' umschreiben läßt. Rückschlüsse auf die Glaubwürdigkeit einer Aussage sollten aus der Persönlichkeit des Zeugen, seinem Lebenswandel etc. gezogen werden. Da aber auch 'ehrliche' Personen unzutreffende Beobachtungen machen können oder ihre Erinnerungsfähigkeit durch verschiedene Umstände beeinträchtigt werden kann $^{42}$, stieß dieses Konzept der 'allgemeinen Glaubwürdigkeit' in den 60er Jahren verstärkt auf Widerspruch und wurde durch das Konzept einer 'speziellen Glaubwürdigkeit' ergänzt und schließlich abgelöst. ${ }^{43}$ Darunter ist

41 Köhnken (1990), S. 84. Für einen Überblick über die historische Entwicklung vgl. ebd. sowie ausführlicher bei: P. Klepzig: Zur Entwicklung der Erforschung und Beurteilung der Glaubwürdigkeit, in: Kriminalistik und forensische Wissenschaften 73-74/1989, S. 198-203 und Hans-Udo Bender: Merkmalskombinationen in Aussagen. Theorie und Empirie zum Beweiswert beim Zusammentreffen von Glaubwürdigkeitskriterien, Tübingen 1987, S. 44ff.

Vgl. Arne Trankell: Der Realitätsgehalt von Zeugenaussagen. Methodik der Aussagepsychologie, Göttingen 1971, S. 98.

43

Vgl. Köhnken (1990), S. 82f. und Eckhard Littmann / Hans Szewczyk: Zu einigen Kriterien und Ergebnissen forensisch-psychologischer Glaubwürdigkeitsbegutachtung von 
"eine Art 'tatbezogene Glaubwürdigkeit"' ${ }^{44}$ zu verstehen, die situative Faktoren in Rechnung stellt. "Damit ist nun nicht mehr in erster Linie die Persönlichkeit des Zeugen, sondern die Aussage selbst und das sie begleitende Verhalten die für die Beurteilung relevante Informationsquelle". ${ }^{45}$ Entsprechend wird nach Merkmalen gesucht, die exklusiv in wahren oder falschen Aussagen auftreten. Betrachtet wird dabei vorwiegend der Inhalt der Aussage; zusätzlich werden konkrete Verhaltensdispositionen und die Aussagefähigkeit der Zeugen geprüft. $^{46}$

\subsection{Glaubwürdigkeitsmerkmale des Aussageinhalts}

Ein zentrales Kriterium für die Glaubwürdigkeit einer Aussage ist ihr quantitativer Detailreichtum:

"Wenn genaue Ortsangaben gemacht werden, Personen in verschiedener Hinsicht beschrieben werden, die Abfolge ihrer Handlungen Schritt für Schritt wiedergegeben wird, Gespräche reproduziert und nicht nur das Kerngeschehen, sondern auch nebensächliche Umstände

sexuell mißbrauchten Kindern und Jugendlichen, in: Forensia 4/1983, S. 55-72, S. 58. Zur Kritik an der 'allgemeinen Glaubwürdigkeit' siehe darüberhinaus insbesondere Udo Undeutsch: Beurteilung der Glaubhaftigkeit von Aussagen, in: ders. (Hrsg.): Handbuch der Psychologie, Band 11: Forensische Psychologie, Göttingen 1967, S. 26-181. Eine ausführliche Darstellung der historischen Entwicklung des Begriffs 'Glaubwürdigkeit' im Strafprozeß liefert Thomas H. Bungardt: Operationalisierung eines Dispositionsbegriffs am Beispiel der Glaubwürdigkeit, Frankfurt a.M. 1981 (Kapitel 3, S. 38-121).

Klepzig (1989), S. 201.

45

Köhnken (1990), S. 83.

46

Vgl. Hans Szewczyk / Eckhard Littmann: Untersuchungen zur Glaubwürdigkeitsbeurteilung kindlicher Zeugen, in: Arne Trankell (Hrsg.): Reconstructing the Past. The Role of Psychology in Criminal Trials, Stockholm 1982, S. 75-103, S. 78ff. und Trankell (1971), S. 99f. Die Delikte, zu denen Zeugenaussagen untersucht werden, sind zu 92 Prozent Sexualdelikte an Kindern, Jugendlichen und Heranwachsenden. (Vgl. Friedrich Arntzen: Die Situation der forensischen Aussagepsychologie in der Bundesrepublik Deutschland, in: Trankell (1982), S. 107-120, S. 108. 
berichtet werden, dann kann man von einem hohen Detaillierungsgrad der Aussage in quantitativer Beziehung sprechen. Eine gegenteilige Ausprägung hat man in der 'pauschalen', undifferenzierten, allgemeingehaltenen Zeugenaussage." ${ }^{47}$

Daneben ist eine Detailierung in qualitativer Hinsicht von Bedeutung. Dazu zählen die Schilderung eigenpsychischer Vorgänge (Angst, Argwohn etc. sowie Affektverläufe), phänomengebundene Schilderungen, bei denen die Beobachtungen nicht in größere Zusammenhänge eingeordnet werden können, und die Schilderung ausgefallener, origineller Einzelheiten ebenso wie die Wiedergabe von Gesprächen aus unterschiedlichen Rollen und Interaktionsschilderungen. ${ }^{48}$

Bender verweist zusätzlich auf Selbstbelastungen, die ohne Notwendigkeit in "situativer Freiwilligkeit" erfolgen und gibt zwei "Phantasiekriterien" an, die Hinweise auf Unglaubwürdigkeit liefern: die übertriebene Wahrheitsbeteuerung und Freud'sche Versprecher, die er charakterisiert als "Aussageeigenarten, bei denen die Wortwahl entweder in offenem Widerspruch zum Inhalt der Aussage oder mindestens ungereimt ist." ${ }^{49}$

Unmittelbar aus dem Inhalt läßt sich auch die Homogenität der Aussage erschließen.

"Wir verstehen hier unter der Homogenität einer Aussage, daß sich in ihr inhaltliche Details zu einem Ganzen ohne Unstimmigkeiten zusammenschließen, daß deren Einzelheiten in einen

$47 \quad$ Friedrich Arntzen: Psychologie der Zeugenaussage. System der Glaubwürdigkeitsmerkmale, München ${ }^{3} 1993$, S. 27. Ähnlich äußert sich Bender (1987: 92). Auch Trankell (1971: 123) verweist darauf, daß die Wiedergabe wirklicher, nicht erfundener Beobachtungen mit größerem Detailreichtum verknüpft ist, wobei auch die Art der Details aufschlußreich ist: so erhärtet die Schilderung von Details, die für das Geschehen selbst bedeutungslos sind, die Glaubwürdigkeit.

48 Vgl. Arntzen (1993), S. 29ff. Daneben werden noch speziellere oder seltener auftretende Formen benannt, die hier nicht näher ausgeführt werden sollen. Ausgefallene Einzelheiten sieht auch Trankell (1971: 126) als bedeutsam an: "Wenn eine Aussage einzigartige Details enthält, vermehren sich die Gründe, sie als Bekundung eines realen Geschehens zu betrachten." Laut Undeutsch (1967: 138) gewährleisten sie gar "höchste Garantie für die Qualität des Berichtes".

Vgl. Bender (1987), S. 93. 
integrierten organischen Zusammenhang gebracht werden können und daß der Inhalt der Aussage damit auch folgerichtig ist." ${ }^{50}$

Neben dieser 'inneren' Homogenität ist auch eine Homogenität im weiteren Sinne zu berücksichtigen, die auf eine Übereinstimmung mit aussageexternen Erkenntnissen zielt. Solche 'äußere' Homogenität liegt vor, wenn andere Zeugen inhaltlich dekungsgleiche Aussagen liefern oder objektive Sachbefunde, wie etwa gesicherte medizinische Erkenntnisse, kriminaltechnische Feststellungen oder Schriftstücke vorliegen. Insbesondere "wenn der Zeuge die äußeren Gegebenheiten - und damit Kontrollmöglichkeiten - nicht voraussehen, seine Aussage also nicht nach ihnen ausrichten konnte", ist damit ein verläßliches Glaubwürdigkeitsmerkmal gegeben. ${ }^{51}$ Littmann und Szewczyk, die eine Analyse von 173 Glaubwürdigkeitsgutachten vornahmen, geben allerdings zu bedenken, daß "die 'Widerspruchslosigkeit zu feststehenden Tatsachen' nur vernachlässigenswert selten gefunden [wurde], während sich die Kriterien der Realistik, Stimmigkeit und Originalität, der Detailliertheit usf. als Kriterien bewährt haben." 52

\subsection{Glaubwürdigkeitsmerkmale im zeitlichen Verlauf}

Zusätzlich zu den Kriterien, die sich aus einer einzelnen Aussage ableiten lassen, ergeben sich Hinweise auf die Glaubwürdigkeit eines Zeugen durch den Vergleich von (zwei oder mehr) Aussagen, die zu unterschiedlichen Vernehmungszeitpunkten erfolgten. Bei solchen Sequenzkriterien muß berücksichtigt werden, daß Abweichungen von früheren Aussagen nicht von vorn-

50 Arntzen (1993), S. 50. [Im Original kursiv.] Zum Homogenitätskriterium siehe auch Trankell (1971: 126), Bender (1987: 93f.) sowie die Ausführungen zur inneren Stimmigkeit und Folgerichtigkeit bei Undeutsch (1967).

$51 \quad$ Vgl. Arntzen (1993), S. 51.

$52 \quad$ Littmann / Szewczik (1983), S. 66. 
herein als Indiz für mangelnde Glaubwürdigkeit gewertet werden können:

"Wenn die Veränderungen in einer Abfolge von Aussagen sich in Übereinstimmung befinden mit den Veränderungen, die wir auf Grund unserer Kenntnis des Erinnerungsprozesses zu erwarten Veranlassung haben, vermehren sich die Gründe, die Darstellungen des Zeugen als einen Versuch, ein reales Vorkommnis zu beschreiben, zu betrachten." ${ }^{53}$

Relevant in diesem Zusammenhang sind zum einen Aussageergänzungen, zum anderen die Konstanz der Aussage. Ergänzungen, also spontane Erweiterungen der Aussage, sind, sofern sie keine Widersprüche zu früheren Aussagen darstellen, für sich genommen schon ein Ausweis der Glaubwürdigkeit, da bei Falschaussagen in dem Bemühen sofort zu überzeugen in der Regel keine Aussageteile zurückgehalten werden. ${ }^{54}$ Um eine Aussage als glaubwürdig oder "erlebnisbegründet" einzustufen, müssen die Angaben ferner hinsichtlich einiger zentraler Punkte konstant bleiben. Dazu gehören Schilderungen des Kerngeschehens, die Benennung der unmittelbar beteiligten Handlungspartner und Ortsangaben, die Benennung von Gegenständen, die unmittelbaren Bezug zu den in Frage stehenden Handlungen haben sowie Angaben über die Lichtverhältnisse. $^{55}$

\subsection{Glaubwürdigkeitsmerkmale der Aussageweise}

Neben den angeführten inhaltsorientierten Merkmalen, auf denen das Hauptaugenmerk liegt, werden auch im Bereich der forensischen Aussagepsychologie verhaltensorientierte Merkmale berücksichtigt. Rechnung getragen wird der

$53 \quad$ Trankell (1971), S. 130.

54 Vgl. Arntzen (1993), S. 41f. Eine übereinstimmende Einschätzung gibt Undeutsch (1967), S. 153f.

55 Vgl. Arntzen (1993), S. 55. Die Kriterien Ergänzung und Konstanz werden auch von Bender (1987: 94f.) verwendet, allerdings weniger ausführlich erläutert. 
Gefühlsbeteiligung des Zeugen, wobei sich weniger die Intensität des Gefühlausdrucks als die Ablauffolge qualitativ verschiedener Gefühle, die dem Geschehensablauf mit den dabei aufgetretenen wechselnden Gefühlen entspricht, als aufschlußreich erwiesen hat. ${ }^{56}$ Glaubwürdigkeit äußert sich ferner in einer ungesteuerten Aussageweise, die sich in prompten, impulsiven Antworten äußert und sich im Sprechverhalten niederschlägt:

"Ausdrucksmäßig wahrt der ungesteuert Aussagende, wenn er frei von Hemmungen ist, einen ungezwungenen Blickkontakt; Stimmführung und Mimik sind verschiedengradig - je nach Naturell des Aussagenden - gelöst, gelassen oder lebhaft-impulsiv, empathisch, 'mitschwingend'. Es bietet sich kein starres, verkrampftes, stereotypes Ausdrucksgeschehen." ${ }^{57}$

Zu berücksichtigen ist schließlich, daß insbesondere bei Aussagen von Kindern eine unzusammenhängende, unsystematische Darstellungsweise noch nicht gegen den Wahrheitsgehalt der Aussage spricht. Eine solche Inkontinenz ist in erster Linie auf mangelnde intellektuelle Fähigkeiten zurückzuführen. ${ }^{58}$

"Charakteristisch für die inkontinente Aussageweise, die bei einigermaßen differenzierten Aussagen zum Glaubwürdigkeitsmerkmal wird, ist, daß Aussagen unzusammenhängend, sprunghaft vorgebracht werden, daß 'Fetzen', Bruchstücke eines Sachverhaltes über das Vernehmungsgespräch verstreut sind oder in der Schilderung völlig ungeordnet erscheinen, daß zeitliche Rückgriffe erfolgen, überhaupt zeitlich Aufeinanderfolgendes nicht in chronologischer Reihenfolge berichtet wird, daß Teile des Geschehens häufig zunächst nur gestreift werden und dadurch in der Aussage manches zunächst unverständlich bleibt. Für den Außenstehenden ergibt sich schließlich aber (oft aufgrund nachgreifender Fragen) doch ein geschlossenes Bild, das frei von Unstimmigkeiten ist - für ihn fügen sich die Einzelteile sozusagen zu einem homogenen Mosaikbild zusammen." 59

$56 \quad$ Arntzen (1993), S. 71.

$57 \quad$ Atntzen (1993), S. 74.

$58 \quad$ Arntzen (1993), S. 78.

59 Arntzen (1993), S. 79. [Im Original kursiv.] Vgl. auch: Anita K. Barry: Narrative Style and Witness Testimony, in: Journal of Narrative and Life History 1/1991, S. 281-293. 


\subsection{Persönlichkeitsmerkmale, Motive, individueller Entwicklungsstand}

Schließlich ergeben sich Annäherungen an die quellen- und kontextorientierte Glaubwürdigkeitsforschung dahingehend, daß individuelle Eigenschaften von Personen und insbesondere ihre Motive betrachtet werden. Arntzen macht die Objektivität einer Aussage daran fest, daß sie "nicht an persönliche Interessen gebunden ist, sie vielmehr aus uneigennützigen Motiven des Zeugen erwachsen ist, die nur auf eine zutreffende Schilderung der Zeugenbeobachtung ausgerichtet waren." ${ }^{60}$ Während die ältere Aussagepsychologie persönlichen Eigenschaften und Motiven des Zeugen eine Hauptrolle bei der Ermittlung der Glaubwürdigkeit zuwies, steht heute vorwiegend die Aussage selbst im Blickpunkt. Undeutsch nimmt folgende Gewichtung vor:

"Entwicklungsstand und Persönlichkeitsartung erlauben nur sehr indirekte Schlüsse auf den Wahrheitsgehalt einer konkreten Aussage. Abklärung der Motivlage und die Verfolgung der Geschichte der Aussage führen näher heran. Alle diese Daten stellen aber erst die Vorstufe für die Beurteilung der Glaubhaftigkeit einer konkreten Aussage dar. Unmittelbar zur Beurteilung des Wahrheitsgehaltes einer Aussage leiten über die Beobachtung und die Auswertung des Aussageverhaltens. Das entscheidende Material stellt die Aussage selbst dar in der gegenwärtigen Fassung, die auf der Folie ihrer früheren Fassungen gesehen werden muß." 61

In Sonderfällen, nämlich dann, wenn frühere Aussagen der gleichen Person vorliegen, die erwiesenermaßen richtig oder falsch waren, lassen sich anhand sogenannter Kontrollkriterien Strukturvergleiche vornehmen. ${ }^{62}$

Für die hier vorgestellten Kriterien gilt, daß jedes für sich keinen vollen Beweiswert hat. Vielmehr gilt, "daß im Durchschnitt mindestens drei ein-

\footnotetext{
$60 \quad$ Arntzen (1993), S. 85. [Hervorhebung im Original.]

61 Undeutsch (1967), S. 125. [Hervorhebung im Original.]

62 Vgl. Bender (1987), S. 95. Trankell (1971: 137f.) verwendet die Bezeichnung Isomorphiekriterium.
} 
deutige, qualifizierte Glaubwürdigkeitsmerkmale als Merkmalskomplex gegeben sein müssen", die sich, wenn sie aus verschiedenen Kategorien stammen, "in besonderer Weise gegenseitig" stützen. ${ }^{63}$ Kritisch anzumerken bleibt, daß es "bisher an einer ausreichend präzisen operationalen Definition der Kriterien" mangelt und daß folglich keine "objektive Analyse von Ausdrucksprozessen" erfolgt, sondern lediglich "eine mehr oder minder systematisierte Eindrucksbildung auf seiten des Sachverständigen". ${ }^{64}$

\subsection{Zwischenbilanz}

Anders als bei der verhaltensorientierten Glaubwürdigkeitsforschung steht bei der forensischen Glaubwürdigkeitsbeurteilung nicht der Sprecher selbst im Zentrum der Betrachtung, sondern nahezu ausschließlich das, was er sagt. Eine glaubwürdige Aussage zeichnet sich aus durch quantitativen und qualitativen Detailreichtum und durch innere Homogenität, also Schlüssigkeit und Widerspruchsfreiheit. Die Glaubwürdigkeit wird zusätzlich gestützt durch äußere Homogenität, also die Bestätigung der Aussage durch Aussagen anderer Personen oder durch objektiv ermittelbare Sachverhalte sowie durch eine Reihe von Sequenzkriterien, die insbesondere die Konstanz zentraler Inhalte betreffen, wenn Aussagen eines Sprechers zu verschiedenen Zeitpunkten erfolgen. Erst in zweiter Linie werden Merkmale der Aussageweise sowie Persönlichkeitsmerkmale und Motive zur Beurteilung der Glaubwürdigkeit herangezogen.

Die Kritik an der inhaltsorientierten Glaubwürdigkeitsforschung konzentriert sich vorwiegend auf den Umstand, daß es nicht gelungen ist, die verwendeten Kriterien genügend präzise zu operationalisieren. Für die Verwendung der Kriterien in inhaltsanalytischen Verfahren ist diese Kritik nicht

$63 \quad$ Arntzen (1993), S. 22.

$64 \quad$ Köhnken (1990), S. 111. 
von Belang. Hier geht es schließlich nicht darum, anhand der Aussage eines Sprechers eine Glaubwürdigkeitsbeurteilung vorzunehmen. Vielmehr soll festgehalten werden, wie die Aussage eines Sprechers im Hinblick auf ihre Glaubwürdigkeit von seiten Dritter beurteilt wird. Dafür liefern die Merkmale des Inhalts und des zeitlichen Verlaufs hinreichende Anhaltspunkte. Hingegen sind Merkmale zur Aussageweise sowie zu Persönlichkeitseigenschaften und Motiven in den Bereichen der verhaltens-, quellen- und kontextorientierten Glaubwürdigkeitsbeurteilung sowohl konkreter als auch weit ausführlicher dargelegt. Für die Analyse relevante und brauchbare Überlegungen zur Operationalisierung sollten daher aus diesen Gebieten übernommen werden. 


\section{Wo das Wissen aufhört, fängt der Glaube an: Die Glaubwürdigkeit der Quelle}

Den Untersuchungen, die unter dem Schlagwort 'quellen- und kontextorientierte Glaubwürdigkeitsbeurteilung' subsumiert werden können, ist gemein, daß sie danach trachten, diejenigen Eigenschaften und Fähigkeiten zu bestimmen, die einen glaubwürdigen Kommunikator kennzeichnen. Neben diesem zentralen Interesse werden vereinzelt auch Rückschlüsse von der Kommunikationssituation auf die Glaubwürdigkeit der beteiligten Kommunikatoren gezogen.

Laut Bentele sind in diesem Zusammenhang zwei Forschungsschwerpunkte zu unterscheiden: die eher (kommunikations-)psychologische Tradition, die sich, beginnend in den 40er Jahren, der 'source credibility' widmet, und die kommunikationssoziologische Tradition, deren Aufmerksamkeit seit Ende der 50er Jahre der 'media credibility' gilt. ${ }^{1}$ Dieses Kapitel widmet sich ausschließlich der Betrachtung der 'Quellen' im engeren Sinne, betrachtet also Individuen, Organisationen oder Institutionen gesellschaftlicher Provenienz, die als Primärquellen von Aussagen in Erscheinung treten. Die Sonderrolle der Medien als (Vermittlungs-)Quellen wird in einem eigenen Abschnitt gesondert behandelt.

Vgl. Günter Bentele: Der Faktor Glaubwürdigkeit. Forschungsergebnisse und Fragen für die Sozialisationsperspektive, in: Publizistik 33/1988, S. 406-426, S. $408 f$. 


\subsection{Die Anfänge: Das 'Prestige' der Quelle}

Ausgangspunkt früher Forschungsüberlegungen war die Beobachtung, daß Personen Sachverhalte unterschiedlichster Art in Abhängigkeit davon bewerten, wie diese Sachverhalte von anderen Personen oder Personengruppen beurteilt werden. ${ }^{2}$ In einem anderen Zusammenhang wurde die Relevanz des Faktors 'Prominenz' aufgezeigt. ${ }^{3}$ Seit den 30er Jahren setzte man sich daher nicht zuletzt im Rahmen der Erforschung der Wirkung von Propaganda - damit auseinander, daß manche Quellen mit ihren Aussagen eher in der Lage waren, Meinungsänderungen herbeizuführen als andere. Dieses Phänomen wurde unter dem Schlagwort 'Prestige' diskutiert. In relativ einfachen experimentellen Versuchsanordnungen wurde nachgewiesen, daß sich die Akzeptanz einer Mitteilung verändert, je nachdem, ob diese Mitteilung von einer Person mit hohem oder niedrigem 'Prestige' stammt. ${ }^{4}$

So ließ Lorge seine Versuchspersonen zunächst für eine Reihe von State-

2 Vgl. S. E. Asch: The Doctrine of Suggestion, Prestige, and Imitation in Social Psychology, in: Psychological Review 55/1948, S. 250-276, S. 252.

3 Vgl. Mitchel Saadi / Paul R. Farnsworth: The Degrees of Acceptance of Dogmatic Statements and Preferences for Their Supposed Markers, in: Journal of Abnormal and Social Psychology 29/1934-35, S. 143-150, S. 143. Die Autoren beziehen sich hier auf eine frühere Studie, in der demonstriert wurde, daß die Betrachter eines Bildes dieses "schöner" finden, wenn es vermeintlich von einem bekannten Maler stammt.

4 Siehe etwa Claude Arnett / Helen Davidson / Hallett Lewis: Prestige as a Factor in Attitude Changes, in: Sociology and Social Research 16/1931, S. 49-55; Henry J. Wegrocki: The Effect of Prestige Suggestibility on Emotional Attitudes, in: Journal of Social Psychology 5/1934, S. 384-394; William Michael / Bernard Rosenthal / Michael DeCamp: An Experimental Investigation of Prestige-Suggestion for Two Types of Literary Material, in: Journal of Psychology 28/1949, S. 303-323; Raymond Bernberg: Prestige Suggestion in Art as Communication, in: Journal of Social Psychology 38/1953, S. 23-30; David Cole: 'Rational Argument' and 'Prestige-Suggestion' as Factors Influencing Judgment, in: Sociometry 17/1954, S. 350-354. 
ments angeben, in welchem Ausmaß sie diesen zustimmten oder sie ablehnten. Einige Wochen später wurden ihnen dieselben Statements noch einmal präsentiert; diesmal jedoch wurden für jede Stellungnahme Personen mit unterschiedlichem 'Prestige' als Urheber genannt. Gemessen wurde dann, inwieweit sich die Einschätzung der Statements durch diese Zusatzinformation verändert. ${ }^{5}$

Dabei wurde nicht näher definiert, was unter 'Prestige' zu verstehen sei. ${ }^{6}$ Man behalf sich damit, die Zitate einmal allgemein anerkannten Personen - wie etwa den US-Präsidenten Roosevelt, Lincoln oder Jefferson - zuzuschreiben, und im anderen Fall als Urheber der gleichen Zitate Lenin oder den Vorsitzenden der Kommunistischen Partei in Amerika anzugeben. Wie erwartet erhielten die Statements mehr Zustimmung, wenn sie scheinbar von den anerkannten Personen stammten. Ziel dieser und ähnlicher Untersuchungen war allein nachzuweisen, daß die Nennung von Quellen zu einer Einstellungsänderung führen kann, und mittels eines quantitativen Verfahrens das Vorhandensein eines Faktors 'Prestige' aufzuzeigen. ${ }^{7}$ Es wurde noch nicht hinterfragt, welche Faktoren dazu führen, daß einige Personen über mehr 'Prestige' verfügen als andere. ${ }^{8}$

Neben dem Einfluß einzelner Personen als Quellen wurde auch der Einfluß verschiedener Gruppen geprüft. Der frühe Befund von Moore, daß die Kennzeichnung einer Position als 'Mehrheitsmeinung' größeren Einfluß hat, als wenn

5 Vgl. Irving Lorge: Prestige, Suggestion, and Attitudes, in: Journal of Social Psychology 7/1936, S. 386-402.

6 Auch die operationale Definition von Lurie liefert keine inhaltlichen Anhaltspunkte. Er bestimmt: "The prestige of a certain name or symbol with respect to some experimental group is the change in scale value in certain items which is brought about by attaching the name or symbol to the items." (Walter A. Lurie: The Measurement of Prestige and PrestigeSuggestibility, in: Journal of Social Psychology 9/1938, S. 219-225, S. 221.)[Hervorhebung im Original.] Prestige wird hier schlicht gleichgesetzt mit dem Ausmaß der Meinungsänderung. 'Sympathie' bei Saadi / Farnsworth (1934). 
man dieselbe Position als 'Expertenmeinung' kennzeichnet ${ }^{9}$, erfuhr allerdings bei Kulp eine Widerlegung: Das größte relative 'Prestige' hatte hier die eigene Bezugsgruppe, gefolgt von Experten und einer Laienmehrheit. ${ }^{10}$

\subsection{Experimente im Rahmen der Persuasionsforschung: Die Hauptdimensionen 'Kompetenz' und 'Vertrauenswürdigkeit'}

Die ersten Untersuchungen, die die Glaubwürdigkeit von Quellen regelmäßig berücksichtigen, gehen zurück auf die Yale-Gruppe um Carl Hovland. Die Bedeutung der Quellen, die hier als Urheber von Aussagen aufgefaßt werden, wird ausdrücklich hervorgehoben:

"Backyard gossips liberally sprinkle the names of respectable sources throughout their rumors. The debater, the author of scientific articles, and the news columnist all bolster their contentions with quotations from figures with prestige. Approval of a statement by highly respected persons or organizations may have much the same positive effect as if they originate it. (...) The examples above suggest the importance of persons, groups or media which can be subsumed under the general category of 'sources'. Differences in effectiveness may sometimes depend upon whether the source is perceived as a speaker who originates the message, an endorser who is cited in the message, or the channel through which the message is transmitted." ${ }^{11}$

Obgleich hier eine klare Unterscheidung getroffen wird zwischen Primärquellen - also Urhebern von Aussagen oder Aussageträgern, die selbst sprechen oder von Dritten zitiert werden - und Medien, die die Aussagen weiterleiten, wird diesem Unterschied im Verlauf der durchgeführten Untersuchungen keine Aufmerksamkeit geschenkt.

9 Vgl. Henry T. Moore: The Comparative Influence of Majority and Expert Opinion, in: American Journal of Psychology 32/1921, S. 16-20.

10 Vgl. Daniel H. Kulp, II: Prestige, as Measured by Single-Experience Changes and Their Permanency, in: Journal of Educational Research 27/1934, S. 663-672, S. 668.

11 Hovland / Janis / Kelley (1953), S. 19. 
Ferner ging es den Forschern nicht in erster Linie um eine systematische Erforschung der Glaubwürdigkeit; vielmehr konzentrierte sich die Fragestellung im Rahmen der Persuasionsforschung darauf, wie Unterschiede in der Glaubwürdigkeit des Kommunikators die Art und Weise beeinflussen, in der Inhalt und Präsentation wahrgenommen und eingeschätzt werden und insbesondere, welche Auswirkungen sich im Hinblick auf die Veränderung von Einstellungen und Überzeugungen ergeben. ${ }^{12}$ 'Glaubwürdigkeit' wird dabei in Anlehnung an Aristoteles als eine Funktion der beiden Dimensionen Kompetenz ('expertness') und Vertrauenswürdigkeit ('trustworthiness') betrachtet.

Diese Vorstellung wird als Faktormodell der Quellenglaubwürdigkeit bezeichnet. Ein Faktor besteht dabei aus einem Bündel wahrgenommener Eigenschaften, die auf die gleiche Sache verweisen: So gehören etwa wahrgenommene Intelligenz, Autorität und die Fähigkeit zu informieren zum Faktor Kompetenz. ${ }^{13}$

Zum besseren Verständnis der Vorgehensweise soll ein typischer Untersuchungsablauf hier kurz skizziert werden: Zwei Gruppen von Versuchspersonen werden mit identischen Texten konfrontiert, wobei die Angaben über die Urheber dieser Texte dahingehend variiert werden, daß je eine glaubwürdige und eine unglaubwürdige Quelle präsentiert wird. Die Meinung der Versuchspersonen zum behandelten Thema wird sowohl vor als auch nach der Textvorlage mittels eines Fragebogens erhoben. Veränderte Meinungen oder Einstellungen zum Gesprächsgegenstand werden dann auf die unterschiedliche Glaubwürdigkeit der Quellen zurückgeführt. ${ }^{14}$

12 Vgl. Hovland / Janis / Kelley (1953), S. 19. Siehe auch: Carl I. Hovland / Walter Weiss: The Influence of Source Credibility on Communication Effectiveness, in: Public Opinion Quarterly 15/1951, S. 635-650.

13 Vgl. Infante / Rancer / Womack (1993), S. 189.

14 Alternativ wird die Einstellung manchmal nur nach der Vorlage des Textes erhoben; die Befunde werden dann mit denen einer Kontrollgruppe aus der gleichen Population verglichen. Unterschiede werden ebenfalls auf die Manipulation der Glaubwürdigkeit zurückgeführt. Vgl. etwa: Elliott McGinnies: Studies in Persuasion IV. Source Credibility and Involvement as 
In der bekanntesten Studie von Hovland/Weiss wurde zu vier Themenkomplexen jeweils eine zustimmende und eine ablehnende Version produziert, die jeweils einmal einer glaubwürdigen und einer eher unglaubwürdigen Quelle zugeschrieben wurde. Folgende Themen und Quellen wurden verwendet: ${ }^{15}$

\begin{tabular}{|c|c|c|}
\hline \multicolumn{2}{|c|}{$\begin{array}{l}\text { Quelle mit hoher } \\
\text { Glaubwürdigkeit }\end{array}$} & $\begin{array}{l}\text { Quelle mit niedriger } \\
\text { Glaubwürdigkeit }\end{array}$ \\
\hline \multicolumn{3}{|l|}{ Thema } \\
\hline \multicolumn{2}{|c|}{$\begin{array}{l}\text { A.Sollen AntihistamineNew England Journal } \\
\text { weiterhin rezeptfrei of Biology and Medicine } \\
\text { verkauft werden? }\end{array}$} & Publikumszeitschrift \\
\hline $\begin{array}{l}\text { B.Ist es möglich, ein atom- } \\
\text { betriebenes } U \text {-Boot zu } \\
\text { bauen? }\end{array}$ & Robert J. Oppenheimer & Pravda \\
\hline $\begin{array}{l}\text { C.Ist die Stahlindustrie } \\
\text { schuld an der derzeitigen } \\
\text { Stahlknappheit? }\end{array}$ & $\begin{array}{l}\text { Bulletin of National } \\
\text { Resources Planning } \\
\text { Board }\end{array}$ & $\begin{array}{l}\text { ein 'rechter', anti-gewerk- } \\
\text { schaftlicher Zeitungs- }\end{array}$ \\
\hline $\begin{array}{l}\text { D. Wird sich die Anzahl der } \\
\text { Kinos bis } 1955 \text { wegen des } \\
\text { Fernsehens verringern? }\end{array}$ & Fortune & \\
\hline
\end{tabular}

Als 'Quellen' fungieren in diesem Experiment sowohl einzelne Personen als auch Medien unterschiedlicher Art. Der Begriff der 'Quelle' wird also undifferenziert angewendet einerseits auf die Urheber von Aussagen, die man als 'Primärquellen' bezeichnen könnte und andererseits auf Medien, die als 'Vermittlungsquellen' die Aussagen anderer weiterleiten. Für beide Arten von

Factors in Persuasion with Students in Taiwan, in: Journal of Social Psychology 74/1968, S. 171-180.

15 Vgl. Hovland/Weiss (1951), S. 637. Eine ähnliche Versuchsanordnung benutzt Thomas D. Cook: Competence, Counterarguing, and Attitude Change, in: Journal of Personality 37/1969, S. 342-358. Die Unterschiede zwischen den Quellen - auch hier werden (Fach-)Zeitschriften und Institutionen verwendet - werden von ihm jedoch ausschließlich als Unterschiede hinsichtlich der Kompetenz bezüglich des Themas interpretiert. Die Frage nach der Vertrauenswürdigkeit wird nicht gestellt. 
Quellen wird als Synonym auch der Begriff 'Kommunikator' benutzt.

Die Befunde zeigten, daß die Quellen mit hoher Glaubwürdigkeit signifikant häufiger eine Meinungsänderung herbeiführten, und zwar unabhängig davon, in welcher Richtung sie argumentierten. Zudem wurden die Textbeiträge, bei denen die Quellen als weniger glaubwürdig eingestuft wurden, als weniger fair und ihre Schlußfolgerungen als weniger gerechtfertigt betrachtet. Lediglich beim letzten Thema (Zukunft des Kinos) führte die Quelle mit niedriger Glaubwürdigkeit einen etwas größeren Prozentsatz an Meinungsänderung herbei. Dagegen ergaben sich in keinem Fall Unterschiede hinsichtlich der Behaltensleistung. ${ }^{16}$

Hovland/Weiss gaben ihren Probanden abgesehen von der Namensnennung keine weiteren Hinweise auf Kompetenz oder Vertrauenswürdigkeit der Quellen. Offenbar nahm man als gegeben an, daß die Kompetenz der Quellen mit hoher Glaubwürdigkeit (ein Wissenschaftler, die Veröffentlichung einer staatlichen Institution und zwei Fachzeitschriften) erkannt wurde. Bei den Quellen mit niedriger Glaubwürdigkeit finden sich zumindest zwei Stimmen, die deutlich von Parteilichkeit geprägt sind (Prawda und ein "rechter" Zeitungskolumnist) und deren Vertrauenswürdigkeit darunter leidet, daß die "Reinheit" ihrer Motive angezweifelt werden kann. Diese Schlußfolgerungen wurden den Versuchspersonen jedoch selbst überlassen.

Andere Studien betonen die Dimensionen 'Kompetenz' und 'Vertrauenswürdigkeit' dadurch, daß sie über die Namensnennung hinaus Zusatzinformationen über die Quellen liefern. Zum Beispiel geben Johnson/Scileppi folgende Beschreibungen vor: Die erste Quelle ist eine medizinische Autorität, ein Experte auf dem Gebiet des Röntgens und der Tuberkulose; die zweite Quelle ist ein Quacksalber, der schon wegen Betrugs im Gefängnis saß, der zum Thema nichts Substantielles beizutragen weiß und der den vorliegenden Text für ein sensationslastiges Magazin geschrieben hat. Der (in beiden Versuchssituationen identische) Text beschäftigt sich mit den Gefahren von Röntgen-

16 Vgl. Hovland / Weiss (1951), S. 640ff. 
strahlen. ${ }^{17}$ Während bei der ersten Quelle ausschließlich die Kompetenz betont wird, werden bei der zweiten deutliche Abstriche hinsichtlich beider Dimensionen 'Kompetenz' und 'Vertrauenswürdigkeit' gemacht. ${ }^{18}$ Auch hier ergibt sich, daß die präsentierten Argumente dann akzeptiert werden und zu Meinungsänderungen führen, wenn sie von der glaubwürdigen Quelle stammen.

Dieses Grundkonzept bei der Forschungsanlage - eine standardisierte Mitteilung, die von verschiedenen Personen mit je unterschiedlicher Glaubwürdigkeit vorgetragen wird - ist einer Vielzahl von Studien gemein. ${ }^{19}$ Die Merkmale für Glaubwürdigkeit werden dabei von den Forschern festgelegt. In der Mehrzahl der Studien wird implizit unterstellt, daß die Glaubwürdigkeit des Sprechers abhängig ist von dem Thema, über das er spricht. ${ }^{20}$ Das bedeutet,

17 Vgl. Homer H. Johnson / John A. Scileppi: Effects of Ego-Involvement Conditions on Attitude Change to High and Low Credibility Communicators, in: Journal of Personality and Social Psychology 13/1969, S. 31-36, S. 32.

18 Zusätzlich wird im Fall der zweiten Quelle auch noch die Glaubwürdigkeit einer Vermittlungsquelle herabgesetzt, indem darauf verwiesen wird, daß es sich um ein Magazin handelt, das zu Sensationsdarstellungen neigt.

19 Siehe z.B. Elliot Aronson / Burton W. Golden: The Effect of Relevant and Irrelevant Aspects of Communicator Credibility on Opinion Change, in: Journal of Personality 30/1962, S. 135-146; Bradley S. Greenberg / Gerald R. Miller: The Effects of Low-Credible Sources on Message Acceptance, in: Speech Monographs 33/1966, S. 127-136; Elaine Walster / Elliot Aronson / Darcy Abrahams: On Increasing the Persuasiveness of a Low Prestige Communicator, in: Journal of Experimental Social Psychology 2/1966, S. 325-342; C. Neil Macrae / John W. Shepherd / Alan B. Milne: The Effects of Source Credibility on the Dilution of Stereotype-Based Judgments, in: Personality and Social Psychology Bulletin 18/1992, S. 765-775. In der Regel wird bei der Operationalisierung der verschiedenen Glaubwürdigkeitsstufen eine qualitative Veränderung der Eigenschaften vorgenommen. Es gibt aber auch Versuche mit quantitativen Variationen: So operationalisieren BEACH et al. hohe und niedrige Glaubwürdigkeit durch die Variation des Prozentsatzes, der besagt, in welchem Ausmaß eine Quelle früher schon zutreffende Aussagen gemacht hat. (Vgl. Lee Roy Beach et al.: Information Relevance, Content and Source Credibility in the Revision of Opinions, in: Organizational Behavior and Human Performance 21/1978, S. 1-16. S. 3.)

20 Vgl. Kenneth Andersen / Theodore Clevenger Jr.: A Summary of Experimental Research in Ethos, in: Speech Monographs 30/1963, S. 59-78, S. 60. 
daß jemand, der als Experte bezüglich eines Sachgebiets gilt, hohe Glaubwürdigkeit hat, wenn er ein Thema behandelt, das in sein Sachgebiet fällt. Dagegen wird Glaubwürdigkeit nicht automatisch schon zugeschrieben, wenn ein Sprecher über ausgeprägte Kompetenzmerkmale verfügt, aber zu einem Thema Stellung nimmt, dessen Behandlung durch diese Kompetenzmerkmale nicht abgedeckt ist. Dieser Zusammenhang von Kompetenz und Thema wird auch als 'Kompetenzrelevanz' ('relevance of expertness') bezeichnet. ${ }^{21}$

Ein weiteres gemeinsames Merkmal dieser Untersuchungen ist, daß Glaubwürdigkeit als feststehendes Merkmal der Quelle betrachtet wird, das sich im Verlauf der Kommunikation nicht ändert. Demgegenüber existiert in späteren Studien - in erster Linie in solchen, die mit dem Semantischen Differential arbeiten - die Vorstellung, daß sich im Verlauf der Kommunikation Veränderungen des Sprecherimages und also auch seiner Glaubwürdigkeit einstellen können, die verursacht sind von den Aussagen des Sprechers oder von situativen Faktoren. ${ }^{22}$

Laut Bentele ist die lerntheoretisch fundierte sozialpsychologische Konzeption der Hovland-Studien aus heutiger Sicht zu einfach. Erfahrungen des Rezipienten, seine individuelle Informationslage oder soziodemographische Daten werden nicht berücksichtigt; Glaubwürdigkeit wird "vor allem als objektiver Faktor des Kommunikators betrachtet, nicht als subjektiver (zugeschriebener) Faktor innerhalb des Rezeptionsprozesses." ${ }^{23}$ Letzterer Kritikpunkt kann allerdings nicht nachvollzogen werden; in den meisten Fällen wird durchaus klar, daß wahrgenommene und nicht tatsächliche Glaubwürdigkeit

\footnotetext{
21 Vgl. Vernon A. Stone / James L. Hoyt: The Emergence of Source-Message Orientation as a Communication Variable, in: Communication Research 1/1974, S. 89-109 [im folgenden zitiert als 1974b]. Siehe dazu auch: David K. Berlo / James B. Lemert / Robert J. Mertz: Dimensions for Evaluating the Acceptability of Message Sources, in: Public Opinion Quarterly 33/1969, S. 563-576, S. 566. Die Forscher meinen den gleichen Zusammenhang, wenn sie von "sources in a relevant context" sprechen.

22 Vgl. Andersen / Clevenger (1963), S. 60

23 Bentele (1988), S. 410.
} 
gemessen werden soll. Zu dieser Interpretation gelangen auch Infante/ Rancer/Womack:

"According to the factor model, source credibility is represented by how favourably the receiver judges the source on each of the factors of credibility. Thus, credibility exists in the mind of the receiver; it is not an actual characteristic of the source like eye color or hair color. If a source has an I.Q. of 160, is a published author on the subject of her speech, and presents an enormous amount of information on that topic, that does not mean that the audience will necessarily view her as an expert. One person may consider the source an expert on the topic, while another person may not. Credibility is strictly in the eye of the beholder." 24

Berlo/Lemert/Mertz verweisen ebenfalls auf den Zuschreibungscharakter, konstatieren allerdings, daß der Begriff 'source credibility' mißverständlich sei. Sie entscheiden sich für eine Umbenennung, um eben diesen Zuschreibungscharakter zu verdeutlichen:

"Because of the restrictive and ambiguous meanings attached to the label 'source credibility' ... and the tendency for such labels to suggest that the variable is the property of the source rather than a receiver's response to a source, we have chosen to refer to the construct, rather unimaginatively, as 'dimensions for evaluating message sources'." 25

Einschränkend merkt Köhnken zu den Untersuchungen zur Quellenglaubwürdigkeit an, daß in diesen Fällen die Glaubwürdigkeit des 'Kommunikators' nicht direkt untersucht wird, sondern daß lediglich bestimmte Informationen über die Quelle gegeben werden, von denen die Forscher annehmen, daß sie die gewünschte Beurteilung bewirken. Auch die zugeschriebene Glaubwürdigkeit wird in der Regel nicht gemessen; vielmehr wird anhand der festgestellten Meinungs- oder Einstellungsänderung auf die Wirksamkeit der Attribution ge-

24 Infante / Rancer / Womack (1993), S. 189. Ähnlich äußern sich auch Richard D. Rieke / Malcolm O. Sillars: Argumentation and the Decision Making Process, New York u.a. 1975, S. 141.

25 Berlo / Lemert / Mertz (1969), S. 565. 
schlossen. $^{26}$

Trotz der Vielzahl der Studien fehlen klare und systematische Kriterien zur Operationalisierung der beiden Dimensionen 'Kompetenz' und 'Vertrauenswürdigkeit'. Gesicherte Schlußfolgerungen lassen sich lediglich in Teilbereichen ziehen. So ergibt sich für die 'Kompetenz' übereinstimmend, daß formelle Titel (z.B. Professor) oder die Bezeichnung einer Quelle als 'Experte für ... ' als Kriterien herangezogen werden können. Ob damit dieser Bereich schon vollständig erfaßt ist, bleibt offen. Ungeklärt ist nämlich, was derlei Titel oder Bezeichnungen dem Rezipienten signalisieren. (Fach-)Wissen, Erfahrung, laut Hovland et al. auch allgemein Intelligenz und "gut informiert sein" ${ }^{27}$ sowie Insider-Wissen, Qualifikation oder Begabung, Geschick und Können sind hier denkbare Schlagwörter, die den Bereich 'Kompetenz' konkretisieren. Die Wahrnehmung von 'Kompetenz' kann zusätzlich beeinflußt sein durch das Alter der Quelle (je nach Kultur kann Alter mit Erfahrung gleichgesetzt werden) oder durch das Innehaben von Status oder Führungspositionen. ${ }^{28}$

Noch weitaus ungesicherter erscheint auf der Basis dieser Untersuchungen, welche Faktoren für das Zustandekommen von 'Vertrauenswürdigkeit' verantwortlich sind. Zum Teil werden die Quellen nicht mit Merkmalen versehen, die explizit auf diese Dimension verweisen. Offenbar wird unterstellt, daß Kompetenzmerkmale zugleich geeignet sind, Vertrauen zu erzeugen. In anderen Fällen wird die 'Vertrauenswürdigkeit' durch Hinweise auf Ehrlichkeit oder Aufrichtigkeit hergestellt. Zuverlässigkeit ${ }^{29}$, Beständigkeit, Seriosität,

26

Vgl. Köhnken (1990), S. 123. Lediglich Hovland/Weiss (1951) fragten ihre Probanden ausdrücklich nach der Vertrauenswürdigkeit der Quellen, so daß gesichert war, daß sich die Einschätzung der Forscher mit der der Versuchspersonen deckte; die Dimension Kompetenz wurde allerdings nicht geprüft.

27 Vgl. Hovland / Janis / Kelley (1953), S. 21.

28

Vgl. ebd. S. 22. Siehe auch: Herbert Goldberg / Marvin A. Iverson: Inconsitency in Attitude of High Status Persons and Loss of Influence: An Experimental Study, in: Psychological Reports 16/1965, S. 673-683.

29

Vgl. etwa die Definition von Rotter, der Vertrauen als die Erwartung bezeichnet, "that the word, promise, verbal or written statement of another individual or group can be relied on". 
Spontaneität sowie die Übereinstimmung von Reden und Taten sind weitere denkbare Konkretisierungen.

Eine Komponente der 'Vertrauenswürdigkeit', deren Einfluß geprüft wurde, ist die von Aristoteles postulierte "Reinheit der Motive". Es gilt als gesichert, daß Quellen, von denen bekannt ist, daß sie bestimmte Interessen oder Absichten verfolgen, weniger glaubwürdig erscheinen. So zeigte sich, daß identische Kommunikationsinhalte unterschiedlich wahrgenommen werden, wenn einer der Quellen Parteilichkeit unterstellt wird. Die Aussagen der unparteilichen Quelle wurden als neutraler eingestuft, die Quelle selbst als fairer und ehrlicher. ${ }^{30}$ Wenn parteiliche Quellen allerdings gegen ihre vermuteten Eigeninteressen argumentieren oder Auffassungen vertreten, die man von ihnen nicht erwartet, wirken sie glaubwürdiger und führen eine stärkere Meinungsänderung herbei. ${ }^{31}$

(Julian B. Rotter: Generalized Expectancies for Interpersonal Trust, in: American Psychologist 26/1971, S. 443-452, S. 444.)

30 Vgl. dazu Carl I. Hovland / Wallace Mandell: An Experimental Comparison of Conclusion-Drawing by the Communicator and by the Audience, in: Journal of Abnormal and Social Psychology 47/1952, S. 581-588, S. 584 oder Herbert C. Kelman / Carl I. Hovland: Reinstatement of the Communicator in Delayed Measurement of Opinion Change, in: Journal of Abnormal and Social Psychology 48/1953, S. 327-335. Zur Bedeutung der Motive der Quelle siehe auch: Jack McKillip: Credibility and Impression Formation, in: Personality and Social Psychology Bulletin 1/1975, S. 521-524.

31 Vgl. Walser / Aronson / Abrahams (1966) sowie Gary F. Koeske / William D. Crano: The Effect of Congrous and Incongrous Source-Statement Combinations upon the Judged Credibility of a Communication, in: Journal of Experimental Social Psychology 4/1968, S. 384399; Paul L. Kohn / Suzi Snook: Expectancy-Violation, Similarity, and Unexpected Similarity as Sources of Credibility and Persuasiveness, in: Journal of Psychology 94/1976, S. 185-193. und R. W. McPeek / J. D. Edwards: Expectancy Disconfirmation and Attitude Change, in: Journal of Social Psychology 96/1975, S. 193-208. Die Erwartung eines Rezipienten, daß ein Kommunikator gemäß seinen eigenen Interessen argumentiert, wird von EAGLY et al. als "knowledge bias" bezeichnet. Sie belegen zusätzlich einen "reporting bias", der meint, daß für gewöhnlich davon auszugehen ist, daß Sprecher danach trachten, ihrem Publikum 'nach dem Munde zu reden'. Für beide Erwartungen gilt, daß ihre Nichterfüllung dazu führt, daß Rezipienten sich eher überzeugen lassen und den Sprecher als aufrichtiger einstufen. (Vgl. Alice H. Eagly / Wendy Wood / Shelly Chaiken: Causal Inferences About Communicators and Their Effect on Opinion Change, in: Journal of Personality and Social Psychology 36/1978, S. 424-435.) Ein Verstoß gegen die Erwartungen kann bei bestimmten Personen aber auch dazu führen, daß das Vertrauen 
Um Vertrauenswürdigkeit zu erzeugen, gibt es - abgesehen von der überwiegend verwendeten Zuschreibung von bestimmten, meist stabilen Eigenschaften der Kommunikationsquelle - noch die Möglichkeit, "daß der Kontext, in dem die Äußerung angeblich erfolgte, eine objektive oder verzerrte Darstellung nahelegt." 32 Der Kommunikationskontext kann dabei auf zwei Arten manipuliert werden, die sich mit den Schlagworten 'Forewarning' und 'Overhearing' umreißen lassen. 'Forewarning' steht für eine 'Warnung' des Rezipienten: die Beeinflussungsabsicht der Quelle wird explizit betont. ${ }^{33}$ Dabei wird davon ausgegangen, daß das Wissen um gezielte Beeinflussungsversuche die Vertrauenswürdigkeit der Quelle mindert und die Rezipienten entsprechend weniger ihre Meinungen und Einstellungen ändern.

Es gibt jedoch auch Bedingungen, die den Forewarning-Effekt außer Kraft setzen: Mills und Aronson zeigten, daß männliche Zuhörer sich von einer weiblichen Quelle deutlich stärker beeinflussen ließen, wenn die Quelle attraktiv war und ihre Beeinflussungsabsicht kundtat. ${ }^{34}$ Mills wies ferner nach, daß besonders starke Meinungsänderungen erreicht werden konnten, wenn zusätzlich zur Beeinflussungsabsicht mitgeteilt wurde, daß der Kommunikator

in die Quelle sinkt: "While open-minded individuals were relatively unaffected by source expectancies, close-minded persons rejected both him and his messages when their expectations were disconfirmed." (James Rotton / Brian F. Blake / Richard Heslin: Dogmatism, Trust, and Message Acceptance, in: Journal of Psychology 96/1977, S. 81-88, S. 87.)

Köhnken (1990), S. 121. [Hervorhebung im Original.]

Vgl. William J. McGuire / Demetrios Papageorgis: Effectiveness of Forewarning in Developing Resistance to Persuasion, in: Public Opinion Quarterly 26/1962, S. 24-34; Charles A. Kiesler / Sara B. Kiesler: Role of Forewarning in Persuasive Communications, in: Journal of Abnormal and Social Psychology 68/1964, S. 547-549; R. G. Hass / K. Grady: Temporal Delay, Type of Forewarning and Resistance to Influence, in: Journal of Experimental Social Psychology 11/1975, S. 459-469; Robert Dean / John Austin / William Watts: Forewarning Effects in Persuasion: Field and Classroom Experiments, in: Journal of Personality and Social Psychology $18 / 1971$, S. $210-221$. Attractiveness and Desire to Influence, in: Journal of Personality and Social Psychology 1/1965, S. $173-177$. 
sein Publikum mag. ${ }^{35}$ Möglicherweise gelangten die Probanden durch diese Sympathiebekundung (die nicht durch den Kommunikator selbst erfolgte) zu der Überzeugung, daß dieser sich besonders für ihre Interessen einsetzt. Bedeutsam ist auch das Thema der Aussage: Bei Themen, die für den Rezipienten wichtig sind, wiegt die Beeinflussungsabsicht schwerer als bei eher unwichtigen Themen. ${ }^{36}$

"Zusammenfassend können wir festhalten, daß mit dem Paradigma der expliziten Warnung vor Beeinflussungsversuchen keine eindeutigen Glaubwürdigkeitseffekte nachgewiesen werden konnten. Zwar kann die Unterstellung einer Beeinflussungsabsicht Auswirkungen auf die dem Kommunikator und seinen Äußerungen zugeschriebene Aufrichtigkeit haben, doch scheint dieser Effekt durch eine Reihe zusätzlicher Faktoren wie insbesondere die persönliche Bedeutung der angesprochenen Thematik moderiert zu sein. Andererseits können unter bestimmten Randbedingungen sogar gegenteilige Effekte eintreten, d.h. ein Kommunikator kann größere Persuasionswirkungen erzielen, wenn er seine Beeinflussungsabsicht offen zu erkennen gibt." ${ }^{37}$

Während beim 'Forewarning' also ausdrücklich eine beeinflussende Absicht bekundet wird, ist das Kennzeichen einer 'Overhearing'-Versuchsanordnung, daß jede derartige Intention ausdrücklich ausgeschlossen erscheint. Die Versuchspersonen werden dabei in eine Situation gebracht, in der sie eine Unterhaltung zufällig belauschen, wobei die Kommunikatoren scheinbar keine Ahnung von ihrer Zuhörerschaft haben. Beeinflussungseffekte sind auch hier stärker, wenn das Thema für die Rezipienten persönliche Relevanz hat. ${ }^{38}$

35 Vgl. Judson Mills: Opinion Change as a Function of the Communicator's Desire to Influence and Liking for the Audience, in: Journal of Experimental Social Psychology 2/1966, S. 152-159.

36 Vgl. Richard E. Petty / John T. Cacioppo: Effects of Forewarning on Persuasive Intent and Involvement on Cognitive Responses and Persuasion, in: Personality and Social Psychology Bulletin 5/1979, S. 173-176. [1979a]

37 Köhnken (1990), S. 129f. [Hervorhebung im Original.]

38 Vgl. Elaine Walster / Leon Festinger: The Effectiveness of "Overheard" Persuasive Communications, in: Journal of Abnormal and Social Psychology 65/1962, S. 395-402; T. Brock / L. A. Becker: Ineffectiveness of "Overheard" Counterpropaganda, in: Journal of Personality 
Da sich das Interesse der Forscher auf den Nachweis von Einstellungsänderungen beschränkte, begnügten sie sich meist damit, den Quellen hohe bzw. niedrige Glaubwürdigkeit zuzuschreiben, ohne die Dimensionen 'Kompetenz' und 'Vertrauenswürdigkeit' systematisch zu variieren. Entsprechend lassen sich zunächst keine verläßlichen Aussagen dahingehend ableiten, welche der beiden Komponenten dem Rezipienten bedeutsamer erscheint.

Am Experiment von Hovland/Weiss wurde deutlich, daß mit der Beschränkung auf die Kompetenz der Quelle bereits Meinungsänderungen erreicht werden können - beim ersten Quellenpaar (New England Journal of Biology and Medicine vs. Publikumszeitschrift) gab es keine Hinweise, die die Dimension 'Vertrauenswürdigkeit' berührten. Es lassen sich aber auch Beispiele konstruieren, die zeigen, daß eine Komponente unter Umständen nicht ausreicht, um Glaubwürdigkeit zu erzeugen. So war die Glaubwürdigkeit des schleswig-holsteinischen Ministerpräsidenten Uwe Barschel erst dann nachhaltig erschüttert, als sich herausstellte, daß sein Ehrenwort falsch war. Verletzt war hier ausschließlich die Dimension 'Vertrauenswürdigkeit'; an seiner fachlichen und sachlichen Kompetenz hatte sich nichts geändert. Umgekehrt wird sich wohl jeder davor hüten, den Börsentips eines noch so vertrauenswürdigen Freundes Folge zu leisten, wenn bekannt ist, daß dieser Freund von Geldanlagen nichts versteht.

Ein Versuch, diese beiden Komponenten gegeneinander abzuwägen, wurde von McGinnies und Ward unternommen. Sie legten je einer Gruppe von Probanden eine Quelle mit hoher Kompetenz und hoher Vertrauenswürdigkeit, mit hoher Kompetenz und geringer Vertrauenswürdigkeit, mit geringer Kompetenz und hoher Vertrauenswürdigkeit sowie mit geringer Kompetenz und geringer Vertrauenswürdigkeit vor. Dabei wurden die folgenden Operationalisierungen zugrundegelegt: ${ }^{39}$

and Social Psychology 2/1965, S. 654-660.

39 Vgl. Elliott Mc Ginnies / Charles D. Ward: Better Liked Than Right: Trustworthiness and Expertise as Factors in Credibility, in: Personality and Social Psychology Bulletin 6/1980, S. 467-472, S. $468 f$. 
hoch Westdeutsche Autorität, hat sein

Land auf einer internationalen

Konferenz vertreten;

Artikel zuerst in 'International

Law Review' veröffentlicht.

niedrig Journalist, hat sich vorher

hauptsächlich mit Kunst und

Theater in Westdeutschland

beschäftigt;

Artikel zuerst in Tageszeitung

veröffentlicht.
Wird von seinen Zeitgenossen als ehrlich, aufrichtig und vertrauenswürdig angesehen. Interesse an Gambia bei Urlaub auf eigene Kosten.

Verfasser sympathisiert mit Nazi-Partei; hat unter Journalisten den Ruf verschlagen und berechnend zu sein und seinen persönlichen

stellen. Interesse an Gambia wegen PR-Tätigkeit

Insgesamt am meisten Meinungsänderungen wurden erwartungsgemäß erzielt, wenn die Quelle sowohl Kompetenz als auch Vertrauenswürdigkeit aufwies. Die vertrauenswürdige Quelle konnte jedoch mehr überzeugen, unabhängig davon, ob sie kompetent war oder nicht. Vertrauenswürdigkeit erscheint in dieser Untersuchung also als die wichtigere Dimension. Ob dieser Befund allgemein Gültigkeit hat, muß aber offenbleiben. So ist etwa denkbar, daß allein die Erwähnung der Sympathie für Nationalsozialisten zu so starker Ablehnung führte, daß eine etwaige Kompetenz dadurch überlagert wurde. Die Dominanz der Dimension 'Vertrauenswürdigkeit' wurde allerdings auch in anderen Versuchsanordnungen bestätigt. ${ }^{40}$ In Abwägung der beiden Dimensionen verweist Choo darauf, daß 'Vertrauenswürdigkeit' dann ausschlaggebend für eine Meinungsänderung ist, wenn zwei Kommunikatoren über gleichermaßen

$40 \quad$ Vgl. Louisa Lui / Lionel Standing: Communicator Credibility: Trustworthiness Defeats Expertness, in: Social Behavior and Personality 17/1989, S. 219-221. Hier hörte eine Gruppe von Nonnen einen Vortrag zum Thema AIDS, als dessen Quelle einmal ein Arzt und AIDSSpezialist und einmal ein Priester fungierte. Die Operationalisierung von Vertrauenswürdigkeit wurde hier also ausschließlich über die Gruppenbeziehung vorgenommen. Der Priester wurde als weit glaubwürdiger wahrgenommen. Siehe ferner J[ohn] W. Wanzenried / F. C. Powell: Source Credibility and Dimensional Stability: A Test of the Leathers Personal Credibility Scale Using Perceptions of Three Presidential Candidates, in: Perceptual and Motor Skills 77/1993, S. 403406. 
hohe Kompetenz verfügen; diese Schlußfolgerung ist zwar plausibel, jedoch durch seine eigene Versuchsanordnung nicht gedeckt. ${ }^{41}$

Als Sonderfall einer glaubwürdigen Quelle stellen Levine und Valle Konvertiten heraus, die früheren Überzeugungen oder Handlungsweisen abgeschworen haben. Ihrer Auffassung nach gilt ein "Bekehrter" in besonderem Maße als Experte, weil er persönliche Erfahrung mit der Vertretung "beider Seiten" hat, und als besonders vertrauenswürdig zumindest dann, wenn die "Bekehrung" freiwillig und ohne Zwang erfolgt und kein materieller Gewinn damit verbunden ist. ${ }^{42}$ Der Nachweis besonderer Glaubwürdigkeit wird am Beispiel eines ehemaligen Alkoholikers geführt.

Es ist allerdings fraglich, ob dieser Befund auf alle Konvertiten gleichermaßen übertragen werden kann. ${ }^{43}$ Für einen Fall wie den dort geschilderten, wo jemand Verhaltensweisen ändert, scheint ein Zuwachs an Glaubwürdigkeit plausibel; insbesondere, wenn das frühere Verhalten sozialer Mißbilligung unterworfen ist, wie es beim Alkoholismus zweifellos der Fall ist. Eine andere Konstellation dürfte vorliegen, wenn jemand seine Überzeugungen oder

41 Vgl. Tong-He Choo: Communicator Credibility and Communication Discrepancy as Determinants of Opinion Change, in: Journal of Social Psychology 64/1964, S. 65-76, S. 72. Die verwendeten Quellen sind: "Dr. W. C. Hueper, Head of the Environmental Cancer Section of the National Cancer Institute, Public Health Service" und "Mr. J. P. Richards, Director of Tobacco Industry Public Relation Commitee". Thema der Mitteilung ist der ursächliche Zusammenhang von Rauchen und Lungenkrebs. Obwohl beide Quellen vorab von den Versuchspersonen in eine Kategorie "source likely to know most of the facts" (S. 67) eingeordnet wurden, liegt doch allein aufgrund des akademischen Grads ein eindeutiger Unterschied hinsichtlich der Kompetenzmerkmale vor. Eine Bestätigung der Vermutung findet sich für einen Teilaspekt der Vertrauenswürdigkeit ( Unparteilichkeit) bei Michael H. Birnbaum / Steven E. Stegner: Source Credibility in Social Judgment: Bias, Expertise, and the Judge's Point of View, in: Journal of Personality and Social Psychology 37/1979, S. 48-74. Behaviour and Personality 3/1975, S. 81-90, S. 82.

43 Von anderer Seite wird zu bedenken gegeben, daß die Konstanz von Überzeugungen seitens der Quelle zur Stärkung ihrer Glaubwürdigkeit beiträgt. Vgl. Rotton / Blake / Heslin (1977), S. 82 und Ramon J. Rhine / Robert M. Kaplan: The Effect of Incredulity upon Evaluation of the Source of a Communication, in: Journal of Social Psychology 88/1972, S. 255266. 
Werthaltungen ändert. Ein solcher 'Überzeugungs-Konvertit' wird als besonders glaubwürdig wohl zunächst von denen angesehen, in deren Lager er überwechselt. Dagegen wird die Gruppe oder Partei, die er verläßt, ihn wohl fortan als unglaubwürdig wahrnehmen, weil er durch seinen "Verrat" die Vertrauensdimension grob verletzt hat. Auch unbeteiligte Außenstehende halten jemanden, der seine Gruppe verläßt, weil er eine Mitgliedschaft nicht mehr mit seinen Überzeugungen vereinbaren kann, für glaubwürdiger; er wird als fairer und vertrauenswürdiger eingestuft als ein Gruppenmitglied, allerdings nicht als kompetenter. ${ }^{44}$

\subsection{Methodische Weiterentwicklung: \\ Einsatz des Semantischen Differentials}

Als eine Fortführung der Arbeit von Hovland und seinen Mitarbeitern sehen Berlo/Lemert/Mertz ihre eigenen Forschungen an. Ihnen geht es darum zu prüfen, ob - abgesehen von 'Kompetenz' und 'Vertrauenswürdigkeit' - weitere Dimensionen an der Einschätzung von Glaubwürdigkeit beteiligt sind. Gleichzeitig beschreiten sie methodisch neue Wege, indem sie das Semantische Differential zur Ermittlung dieser Dimensionen heranziehen. Dieses Instrument erscheint ihnen geeignet "because of the similarity of the source evaluation problem to that of the general measurement of connotative meaning." 45

Die Konstruktion der Skalen erfolgte aufgrund vorhergehender Befragungen - Probanden wurden gefragt, welche Quellen sie akzeptieren und warum sowie unter Berücksichtigung von Elementen, die in der Fachliteratur Verwendung fanden. Diese Vorarbeit erbrachte 128 Gegensatzpaare, die zur Beschreibung hoher und geringer Akzeptanz von Quellen geeignet erschienen.

\footnotetext{
44 Vgl. Donald G. Dutton: The Maverick Effect: Increased Communicator Credibility as a Result of Abandoning a Career, in: Canadian Journal of Behavioral Science 5/1973, S. 145-151. 
Die Anpassung an Computerkapazitäten erforderte eine Reduktion auf 83 Paare, wobei darauf geachtet wurde, nur solche Paare zu eliminieren, deren Streichung wegen hoher Bedeutungsähnlichkeit mit im Profil verbleibenden keinen Informationsverlust darstellte. Als Quellen, die der Einschätzung unterworfen waren, wurden bekannte Persönlichkeiten (z.B. Dwight Eisenhower, Winston Churchill, Perry Como), Organisationen (American Medical Association, John Birch Society) und Medien (New York Times, American Broadcasting Company) verwendet. Zusätzlich wurde die Kompetenzrelevanz variiert (z.B.: Chruschtschow spricht einmal über sowjetische Außenpolitik, einmal über moderne Kunst). ${ }^{46}$

Die anschließende Faktorenanalyse erbringt drei Faktoren, die knapp 60 Prozent der gesamten Varianz erklären: 'Safety', 'Qualification' und 'Dynamism'. ${ }^{47}$ 'Safety' und 'Qualification' werden als klarere und umfassendere Version von Hovland's 'Competence' und 'Trustworthiness' aufgefaßt. So beinhaltet 'Safety' neben Eigenschaften, die auf die Absicht der Quelle verweisen (z.B. uneigennützig, fair, gerecht), auch allgemeinere Charaktereigenschaften (wie ruhig, geduldig, freundlich). 'Qualification' umfaßt neben den Kompetenzzuschreibungen auch Aspekte des Prestiges (z.B. wichtig, mächtig, erfolgreich). ${ }^{48}$

Als neue Dimension ergibt sich 'Dynamism', eine Mischung der allgemeinen Faktoren 'Activity' und 'Potency' bei Osgood et al. ${ }^{49}$ Ausdruck dieser

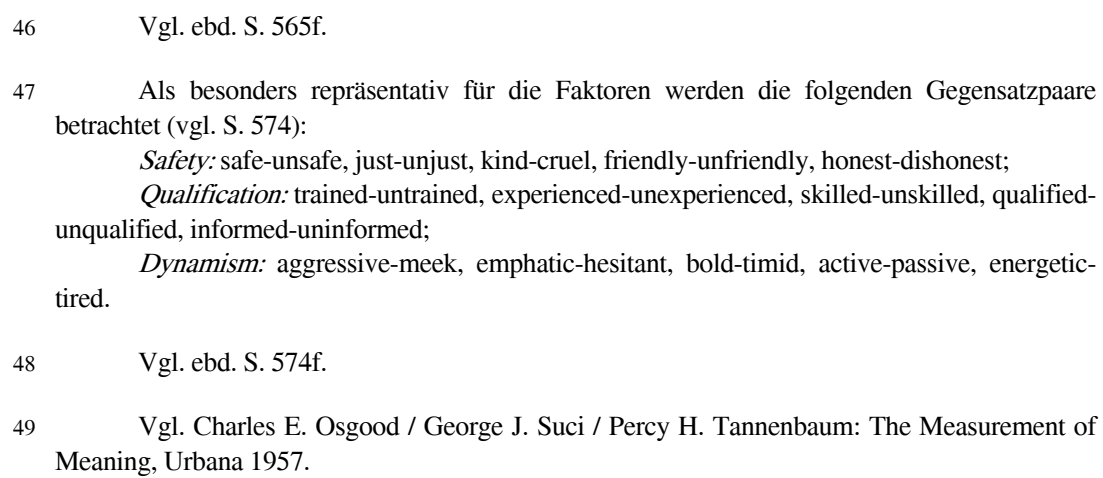


Dimension sind Eigenschaften wie aktiv, aggressiv, extrovertiert, stark und schnell. Der Faktor wird interpretiert als wertende Dimension, die der Quelle zusätzliche "Energie" verleiht:

"Though the results indicate that Dynamism is statistically independent of the Safety and Qualification factors, the relative instability of Dynamism suggests that it may not be psychologically independent of the other two factors. The Dynamism dimension can be conceived of as an intensifier. In other words, given an evaluation of a source as safe or unsafe, qualified or unqualified, the polarity or intensity of these evaluations of the source is intensified through perceptions of high dynamism." 50

Laut eigener Einschätzung der Autoren erhärten die faktoranalytischen Studien die Multidimensionalität der Variable 'Glaubwürdigkeit' und bieten eine operationale Basis, um das 'Image' der Quelle zu definieren. ${ }^{51}$ Eine solche Hinwendung zum 'Image' führt allerdings weg vom Konzept der Glaubwürdigkeit, da das Image einer Person mit Sicherheit noch weitere Komponenten umfaßt. $^{52}$ Eine Gleichsetzung der beiden Begriffe ist eher dazu angetan, der 'Glaubwürdigkeit' Dimensionen zuzurechnen, die keine Bestandteile des Konstrukts sind. Bereits die hier neu gefundene Dimension 'Dynamik' ist mit Vorbehalt zu betrachten, da bei der Sammlung des Materials, das der Skalenkonstruktion zugrundelag, die Probanden nach der Akzeptanz der Quellen gefragt wurden. ${ }^{53}$ Da Akzeptanz auch andere Ursachen als Glaubwürdigkeit haben kann, enthält die Skala wohl eine Reihe von Eigenschaften, die im Verständnis der Rezipienten mit Glaubwürdigkeit nichts zu tun haben, die später aber als Bestandteile der Glaubwürdigkeit interpretiert werden.

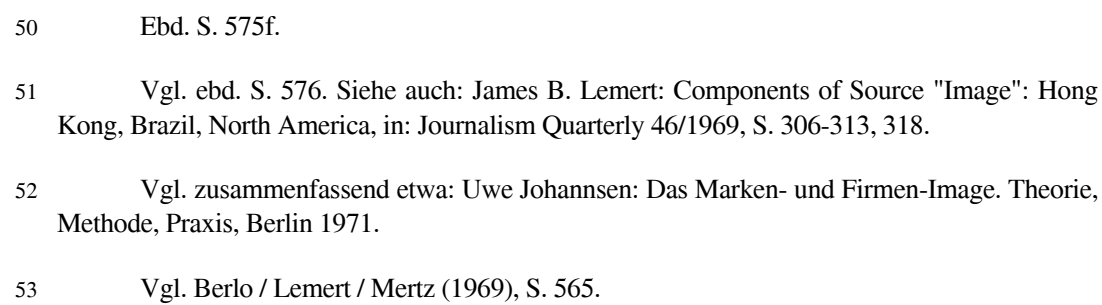


Schweitzer widerspricht der Interpretation von Berlo et al. Seiner Auffassung nach hat der Faktor 'Dynamik' eher additive als intensivierende Funktion und ist somit als eigenständige Komponente der Glaubwürdigkeit zu sehen: "It appears as though being trustworthy or having a good style of presentation alone is not enough to maximize attitude change; rather both assets are needed." ${ }^{54}$ Die Dimension 'Dynamik' umfaßt bei ihm allerdings Elemente, die sich eher auf die Vortragsweise beziehen: anregend vs. schwunglos und stimulierend vs. langweilig. ${ }^{55}$

Unter Bezugnahme auf eine frühere Arbeit von Berlo/Lemert verwenden auch Hewgill/Miller die Dimensionen 'Competence', 'Trustworthiness' und 'Dynamism', wobei ihnen nur je vier Gegensatzpaare als Grundlage dienen. ${ }^{56}$ Hingegen schließt McCroskey 'Dynamism' als signifikanten Faktor aus. Er erhält aus der Faktorenanalyse die Dimensionen 'Authoritativeness' und 'Character', die er mit 'Competence' und 'Trustworthiness' gleichsetzt. Für den dritten theoretischen Faktor 'Good Will' (Aristoteles) findet er keine Belege;

54 Don A. Schweitzer: The Effect of Presentation on Source Evaluation, in: Quarterly Journal of Speech 56/1970, S. 33-39, S. 38. Inwieweit seine eigene Untersuchung diese Interpretation stützt, läßt sich allerdings nicht schlüssig nachvollziehen, da er keine Auskunft darüber gibt, auf welche Weise er die 'Dynamik' des Vortrags bei seinen Quellen manipuliert hat.

55

Vgl. ebd. S. 33f. sowie erstmals und ausführlich bei: Don Schweitzer / Gerald P. Ginsburg: Factors of Communicator Credibility, in: Carl W. Backman / Paul F. Secord (Hg.): Problems in Social Psychology. Selected Readings, New York u.a. 1966, S. 94-102.

56 Vgl. Murray A. Hewgill / Gerald R. Miller: Source Credibility and Response to FearArousing Communications, in: Speech Monographs 32/1965, S. 95-101, S. 97. Sie berufen sich dabei auf: David K. Berlo / James B. Lemert: An Empirical Test of a General Construct of Credibility, paper presented at the SAA Convention, New York Dec. 29, 1961. Die folgenden Gegensatzpaare werden verwendet: incompetent;

Competence: experienced-inexperienced, expert-ignorant, trained-untrained, competent-

Trustworthiness: just-unjust, kind-cruel, admirable-contemptible, honest-dishonest;

Dynamism: aggressive-meek, bold-timid, energetic-tired, extroverted-introverted.

Das gleiche Instrument wird eingesetzt bei: Gerald R. Miller / Murray A. Hewgill: The

Effect of Variations in Nonfluency on Audience Ratings of Source Credibility, in: Quarterly Journal of Speech 50/1964, S. 36-44. 
vielmehr deutet sich an, daß dieser nicht von den beiden anderen Dimensionen $\mathrm{zu}$ trennen ist. ${ }^{57}$ Whitehead ermittelt neben 'Kompetenz', 'Vertrauen' und 'Dynamik' noch den Faktor 'Objektivität' sowie zwölf weitere, die allerdings zusammen nur drei Prozent der Varianz erklären. ${ }^{58}$

Fraglich ist, ob 'Objektivität' tatsächlich als unabhängiger Faktor betrachtet werden kann. Die Nähe von Nennungen wie 'unparteilich' und 'objektiv' zu der Komponente der Vertrauenswürdigkeit, die die 'Reinheit der Motive' umfaßt, ist offensichtlich. Solche Unstimmigkeiten sind in erster Linie auf die Anwendung der Faktorenanalyse zurückzuführen. Das Verfahren ist mit Einschränkungen verbunden, die bei der Betrachtung der Ergebnisse nicht außer acht gelassen werden dürfen. Vor allem ist zu berücksichtigen, daß die Faktoren, die sich bilden, abhängig sind von Art und Anzahl der zugrundeliegenden Semantischen Differentiale: "The genesis of the dimensions which emerge from any factor analytic study is not an unimportant consideration in their acceptance, for no factor analysis can extract factors which are not represented in the original scale items." 59

\subsection{Weitere Dimensionen: Ähnlichkeit, Sympathie und Attraktivität}

57 Vgl. James C. McCroskey: Scales for the Measurement of Ethos, in: Speech Monographs 33/1966, S. 65-72, S. 66.

58 Vgl. Jack L. Whitehead Jr.: Factors of Source Credibility, in: Quarterly Journal of Speech 54/1968, S. 59-63.

59 R. Barry Fulton: The Measurement of Speaker Credibility, in: Journal of Communication 20/1970, S. 270-279, S. 271. Einen ähnlichen Vorwurf richten WAKSHLAG/EDISON an MCCROSKEY: Sie sind der Auffassung, daß sich etwa zusätzlich die Faktoren 'Ähnlichkeit' und 'Sympathie' ergeben hätten, wenn einschlägige Adjektivpaare zur Auswahl gestanden hätten. (Vgl. Jacob J. Wakshlag / Nadyne G. Edison: Attraction, Credibility, Perceived Similarity, and the Image of Public Figures, in: Communication Quarterly 27/1979, S. 27-34, S. 28.) Siehe mit ähnlicher Kritik auch Margaret McLaughlin: Recovering the Structure of Credibility Judgments: An Alternative to Factor Analysis, in: Speech Monographs 42/1975, S. 221-228 und Michael W. Singletary: Components of Credibility of a Favourable News Source, in: Journalism Quarterly 53/1976, S. 316-319, S. 317. 
Bei dem Fall des "Konvertiten" deutete sich bereits an, daß für die Zuschreibung von Glaubwürdigkeit noch weitere Komponenten von Bedeutung sind, die in engem Zusammenhang mit dem Publikum stehen. Eine wichtige Rolle scheint dabei die Ähnlichkeit zwischen Sprecher und Publikum zu spielen. ${ }^{60}$ Sowohl Ähnlichkeiten bezüglich der Gruppenzugehörigkeit als auch Ähnlichkeiten bezüglich Einstellungen und Werthaltungen können dazu beitragen, daß beim Publikum Meinungs- und Einstellungsänderungen stattfinden. ${ }^{61}$ So konnte Adams im Hinblick auf die Gruppenorientierung die Hypothese bestätigen, daß die Meinungsänderungen der Leser in Richtung des Vertreters der eigenen Nation tendieren, wenn zwei Sprecher verschiedener Nationalitäten konfligierende Statements zu einem kontroversen Thema abgeben. ${ }^{62}$ In einer ähnlichen Konstellation werden unter bestimmten Bedingungen auch Einflüsse auf die Glaubwürdigkeit nachgewiesen: Miller/Hoppe zeigten, daß bei anglokanadischen Zuhörern ein anglo-kanadischer Sprecher glaubwürdiger ist als ein franko-kanadischer, jedoch nur, wenn für das Thema, über das gesprochen wird, solche Gruppenbeziehungen relevant sind. ${ }^{63}$ Werbetreibende machen sich diese Einsicht zunutze, indem sie auf sogenannte 'Testmonial'-Formen zurückgreifen, in denen Personen auftreten, die starke Ähnlichkeit mit der

60 Vgl. dazu den Forschungsüberblick von Herbert W. Simons / Nancy N. Berkowitz / R. John Moyer: Similarity, Credibility, and Attitude Change: A Review and a Theory, in: Psycholocical Bulletin 73/1970, S. 1-16 sowie Everett M. Rogers / Dilip K. Bhowmik: Homophily-Heterophily: Relational Concepts for Communication Research, in: Public Opinion Quarterly 34/1970, S. 523-538.

$61 \quad$ Neben diesen beiden Arten von Ähnlichkeit benennt Lowry noch eine "situative" Ähnlichkeit: "a common relationship that the communicator and recipient are in with regard to some past or present situation or experience." (Dennis T. Lowry: Demographic Similarity, Attitudinal Similarity, and Attitude Change, in: Public Opinion Quarterly 37/1973, S. 192-208, S. 195.) Diese Art der Ähnlichkeit wird etwa in Werbespots häufig nahegelegt, ihr Einfluß wurde aber bisher nicht gezielt untersucht.

62 John B. Adams: Effects of Reference Group And Status on Opinion Change, in: Journalism Quarterly 37/1960, S. 408-412.

63 Vgl. Dale T. Miller / Ronald A. Hoppe: The Effect of Regional Similarity-Dissimilarity on Communicator Credibility, in: Language and Speech 16/1973, S. 211-217. 
gewünschten Zielgruppe aufweisen. ${ }^{64}$

Eine Reihe von Studien zeigt, daß ähnliche Werthaltungen von Kommunikator und Rezipient Einfluß auf Meinungsänderungen haben ${ }^{65}$ oder dazu führen, daß der Kommunikator für sympathischer ${ }^{66}$ oder attraktiver ${ }^{67}$ gehalten wird. Häufig wird davon ausgegangen, daß wahrgenommene Ähnlichkeit die Sympathie erhöht und diese verstärkte Sympathie dann ausschlaggebend für

64 Vgl. Roobina Ohanian: Construction and Validation of a Scale to Measure Celebrity Endorsers Perceived Expertise, Trustworthiness, and Attractiveness, in: Journal of Advertising 19/1990, S. 39-52, S. 39. Siehe auch: Ronald E. Milliman / Robert C. Erffmeyer: Improving Advertising Aimed at Seniors, in: Journal of Advertising Research 29/1989-90, S. 31-36.

Vgl. Timothy C. Brock: Communicator-Recipient Similarity and Decision Change, in: Journal of Personality and Social Psychology 1/1965, S. 650-654; Ellen Berscheid: Opinion Change and Communicator-Communicatee Similarity and Dissimilarity, in: Journal of Personality and Social Psychology 4/1966, S. 670-680. Berscheid fand, daß ein Kommunikator mit ähnlichen Werten insbesondere dann überzeugender war, wenn die Werte Relevanz zum behandelten Thema aufwiesen.

66

Vgl. Donn Byrne: Interpersonal Attraction and Attitude Similarity, in: Journal of Abnormal and Social Psychology 62/1961, S. 713-715; Donn Byrne / Don Nelson: Attraction as a Function of Attitude Similarity-Dissimilarity: The Effect of Topic Importance, in: Psychonomic Science 1/1964, S. 93-94; A. J. Smith: Similarity of Values and Its Relation to Acceptance and the Projection of Similarity, in: Journal of Psychology 43/1957, S. 251-260; Jerald M. Jellison / Judson Mills: Effect of Similarity and Fortune of the Other on Attraction, in: Journal of Personality and Social Psychology 5/1967, S. 459-463; Abraham Tesser: Evaluative and Structural Similarity of Attitudes as Determinants of Interpersonal Attraction, in: Journal of Personality and Social Psychology 18/1971, S. 92-96. Der kritische Literaturüberblick von Sunnafrank zeigt allerdings, daß der Zusammenhang von ähnlichen Haltungen und Sympathie nur unter bestimmten Bedingungen gegeben ist und etwa durch persönliche Gespräche Sympathie sich trotz gegensätzlicher Einstellungen einstellen kann. Bochner verneint gänzlich, daß Ähnlichkeit die Sympathie erhöht und plädiert statt dessen für die Hypothese, daß Symathie Einfluß auf wahrgenommene Ähnlichkeit nimmt. (Vgl. Michael Sunnafrank: Interpersonal Attraction and Attitude Similarity: A Communication-Based Assessment, in: James A. Anderson (Hrsg.): Communication Yearbook 14, Newbury Park u.a. 1991, S. 451-483 und Arthur P. Bochner: On the Paradigm That Would Not Die, in: James A. Anderson (Hrsg.): Communication Yearbook 14, Newbury Park u.a. 1991, S. 484-491, S. 487.)

67 Vgl. Manfred Hassebrauck: Der Einfluß von Attitüdenähnlichkeit auf die Beurteilung physischer Attraktivität, in: Zeitschrift für Differentielle und Diagnostische Psychologie 7/1986, S. 89-98; ders.: Perception of Physical Attractiveness Influenced by Similarity of Attitudes, in: Perceptual and Motor Skills 63/1986, S. 185-186. 
Einstellungsänderungen ist. Es gibt aber auch Studien, die zeigen, daß Ähnlichkeiten sich direkt auf Meinungsänderungen auswirken und die den Zusammenhang mit Sympathie ausdrücklich ausschließen. ${ }^{68}$

Während also davon ausgegangen werden kann, daß Ähnlichkeiten zwischen Kommunikator und Rezipient Meinungs- und Einstellungsänderungen direkt oder indirekt - hervorrufen, ist nicht abschließend geklärt, wie sich solche Ähnlichkeiten auf die Wahrnehmung der Glaubwürdigkeit auswirken:

\footnotetext{
"In general, there seems to be a weak but positive relationship between attitudinal similarity and the factors of respect and trust and a still less dependable relationship between membershipgroup similarities and these same factors. In contrast with the extensive body of research linking similarity to attraction, there is scarcely any experimental evidence linking the similarity variable to such other components of a source's image as his apparent honesty, integrity, objectivity, intelligence, competence, etc. Until additional studies are reported, the proposition just stated must be regarded as highly tentative." ${ }^{69}$
}

Wahrscheinlicher ist, daß wahrgenommene Ähnlichkeiten nur unter bestimmten Bedingungen eine Rolle spielen, etwa dann, wenn andere Kriterien fehlen, die für die Einschätzung der Glaubwürdigkeit von Bedeutung sind. Simons et al. machen dies an einem Beispiel deutlich: Dem Professor für Wirtschaft, der zu einem Laienpublikum über internationale Handelsbeziehungen spricht, und der dabei ausdrücklich Hinweise auf seine Kompetenz und Erfahrung gibt, wird dieses Publikum wohl eher Glauben schenken, als einem Laiensprecher, der bekennt, ebensowenig Erfahrung auf dem Gebiet zu haben wie sein Publikum und der auf diese Weise Ähnlichkeiten herausstreicht. ${ }^{70}$ Wenn also manifeste Hinweise auf Kompetenz vorliegen und das behandelte Thema die Gruppe

68 Vgl. Berscheid (1966) sowie James M. Dabbs, Jr.: Self-Esteem, Communicator Characteristics, and Attitude Change, in: Journal of Abnormal and Social Psychology 69/1964, S. 173181, der konstatiert: "Attitude change did not depend on the subjects' liking of the communicator" (S. 178). 
nicht direkt betrifft, wird nach dieser Auffassung die Ingroup-Orientierung nachrangig. Dagegen gewinnt die Ingroup-Orientierung an Wichtigkeit, wenn der Inhalt der Mitteilung für die Gruppe relevant ist; in diesem Fall ist auch die Dimension 'Vertrauenswürdigkeit' berührt. Formale Kompetenz kann dann von nachrangiger Bedeutung sein. ${ }^{71}$ Auf diesen Zusammenhang von Ähnlichkeit und Kompetenz wiesen bereits Hovland et al. hin:

"In certain matters persons similar to the recipient of influence may be considered more expert than persons different from him. An individual is likely to feel that persons with status, values, interests, and needs similar to his own see things as he does and judge them from the same point of view. Because of this, their assertions about matters of which the individual is ignorant but Letztere unterscheiden zwischen "qualification credibility" und "safety credibility". Falls dem Rezipienten in erster Linie daran gelegen ist, Informationen zu sammeln, wird er sich einer qualifizierten aber unähnlichen Quelle zuwenden; wenn es um die Bildung von Einstellungen geht, erfolgt die Orientierung an der ähnlichen Quelle, die "safety credibility" besitzt.

Besonders ausgeprägt ist die Ingroup-Orientierung bei Drogenabhängigen. Das meiste Vertrauen wird Personen entgegengebracht, die ihrerseits Erfahrung mit Drogen haben (andere Drogenkonsumenten oder ehemalige Abhängige). Entsprechend ergibt sich - im Unterschied zu anderen Untersuchungen zur Glaubwürdigkeit - 'Freundschaft' als wichtigster Faktor. Mitglieder aus Gesundheitsberufen werden als signifikant weniger glaubwürdig eingestuft. Zu den am wenigsten vertrauenswürdigen Quellen gehören Medien (mit Ausname von Alternativmedien). Daraus läßt sich die Empfehlung ableiten, bei der Drogenberatung oder bei Hilfsprogrammen neben Experten ehemalige Abhängige einzusetzen. (Vgl. E. Robert Sinnett / Kenneth Hagen / William M. Harvey: Credibility of Sources of Information about Drugs to Heroin Addicts, in: Psychological Reports 37/1975, S. 1239-1242.) Siehe auch E. Robert Sinnett et al.: Credibility of Sources and Information about Drugs, in: Psychological Reports 36/1975, S. 299-309. Die zentrale Rolle der Freundschaft wird auch betont bei Gerhard J. Hanneman: Communicating Drug-Abuse Information among College Students, in: Public Opinion Quarterly 37/1973, 171191. Die geringe Glaubwürdigkeit der Medien für das Thema Drogen wird bestätigt bei Richard Dembo et al:: Drug Information Source Credibility among Junior and Senior High School Youths, in: American Journal of Drug and Alcohol Abuse 4/1977, S. 43-54 und Richard Demo et al:: The Believability of the Media as Sources of Information on Drugs, in: International Journal of the Addictions 12/1977, S. 959-969. Siehe ferner Daniel M. Mayton / Elizabeth A. Nagel / Reese Parker: The Perceived Effects of Drug Messages on Use Patterns in Adolescents, in: Journal of Drug Education 20/1990, S. 305-318; Richard L. Nail / Eric Gunderson: The Youthful Drug Abuser and Drug Abuse Education - Closing the Credibility Gap, in: Journal of Drug Education 5/1975, S. 65-75 sowie den Literaturüberblick bei John E. Buckley: Credibility and Drug Education: A Critique and Reformulation, in: International Journal of the Addictions 24/1989, S. 489-497. 
where he feels the viewpoint makes a difference ... will tend to carry special credibility." ${ }^{72}$

Diese These findet ihre Bestätigung in den Ergebnissen von Jurma, der feststellt, daß bei übereinstimmenden Werthaltungen dem Sprecher höhere Kompetenz zugeschrieben wird; hinsichtlich der Vertrauenswürdigkeit ergeben sich hier zwischen "ähnlichen" und "unähnlichen" Sprechern keine signifikanten Unterschiede. ${ }^{73}$ Vergleichbare Befunde hinsichtlich der Kompetenz liefern auch Eisinger/Mills. Ihnen kam es vor allem darauf an zu prüfen, wie sich das Vertreten von extremen Meinungspositionen auf die Glaubwürdigkeit auswirkt. Das Ergebnis ihres Experiments: Der Kommunikator, der eine Extremposition vertritt, die von der Richtung her mit den Einstellungen der Rezipienten übereinstimmt, wird für kompetenter und aufrichtiger gehalten als ein Kommunikator, der nur ein gemäßigter Fürsprecher der eigenen Seite ist. Ein Kommunikator, der eine Extremposition auf der Gegenseite einnimmt, wird als aufrichtiger aber weniger kompetent beurteilt als einer, der für eine den Rezipienten entgegengesetzte Auffassung von einem moderaten Standpunkt aus eintritt. Vertreter der eigenen Seite werden für kompetenter und allgemein vertrauenswürdiger, nicht aber für aufrichtiger gehalten. ${ }^{74}$ Daraus ist abzulesen, daß Sprecher mit Werthaltungen, die denen des Publikums ähnlich sind, insgesamt für glaubwürdiger gehalten werden als Vertreter gegnerischer

$72 \quad$ Hovland / Janis / Kelley (1953), S. 22.

73 Vgl. William E. Jurma: Evaluations of Credibility of the Source of a Message, in: Psychological Reports 49/1981, S. 778. Bessere Werte hinsichtlich Kompetenz, Attraktivität und Sympathie für Personen mit ähnlichen Werthaltungen ergeben sich auch bei Judson Mills / Charles E. Kimble: Opinion Change as a Function of Perceived Similarity of the Communicator and Subjectivity of the Issue, in: Bulletin of the Psychonomic Society 2/1973, S. 35-36. Vertrauenswürdigkeit wurde in dieser Studie nicht abgefragt.

74 Vgl.Richard Eisinger / Judson Mills: Perception of the Sincerity and Competence of a Communicator as a Function of the Extremity of His Position, in: Journal of Experimental Social Psychology 4/1968, S. 224-232, S. 228ff. Zum Einfluß gleicher Werthaltungen auf die Glaubwürdigkeit vgl. ergänzend auch: Velina Topalova: Credibility of Information Source, in: Polish Psychological Bulletin 5/1974, S. 73-80. 
Positionen. Den Gegnern wird aber - insbesondere, wenn sie extreme Haltungen einnehmen - durchaus zugestanden, daß sie ehrlich sind.

Mills/Jellison gehen hingegen davon aus, daß 'Ähnlichkeit' die Wahrnehmung der Aufrichtigkeit des Kommunikators beeinflußt und prüfen dies anhand von Gruppenzugehörigkeit. ${ }^{75}$ Sie ermitteln zwar stärkere Meinungsänderungen für den Kommunikator aus der gleichen Gruppe, allerdings keine signifikanten Unterschiede hinsichtlich wahrgenommener Charakteristika, die in Zusammenhang mit Glaubwürdigkeit stehen (z.B.: biased, sincere, selfish, competent, likeable). Da die Versuchspersonen aber nur um eine allgemeine Einschätzung gebeten wurden, die sich nicht auf die gehaltene Rede beziehen sollte, messen die Forscher selbst dem Befund keine allzugroße Bedeutung bei. ${ }^{76}$ Denkbar ist schließlich auch, daß die Dimensionen 'Kompetenz' und 'Vertrauenswürdigkeit' in diesem Fall für die Probanden deshalb nicht wichtig waren, weil für das Thema der Rede die Gruppenbeziehung irrelevant war. (Der Redner plädierte für eine bessere Erziehung zur Allgemeinbildung.)

Als ein "idealer" Kommunikator sollte sich demgemäß derjenige erweisen, der grundlegende Übereinstimmungen mit seinem Publikum aufweist und der sich gleichzeitig von ihm abhebt, indem er zusätzlich über Merkmale verfügt, die seine Glaubwürdigkeit erhöhen. ${ }^{77}$ Diese beiden Merkmale sind im 'opinion leader' vereinigt ${ }^{78}$ : Er gehört zum einen der gleichen Gruppe an und ist, was die Aufrechterhaltung zentraler Werte und Normen anbelangt, das "kon-

75 Judson Mills / Jerald M. Jellison: Effect on Opinion Change of Similarity between the Communicator and the Audience He Addressed, in: Journal of Personality and Social Psychology 9/1968, S. 153-156, S. 153.

76Vgl. ebd. S. $155 f$.

77 Zur Bestätigung dieser These siehe etwa Arch G. Woodside / J. William Davenport: The Effect of Salesman Similarity and Expertise on Consumer Purchasing Behavior, in: Journal of Marketing Research 11/1974, S. 198-202. Kompetenz wird hier als die wichtigere Komponente ermittelt; am wirkungsvollsten im Hinblick auf eine Kaufentscheidung ist die Kombination von Kompetenz und Ehrlichkeit. 
formistischste" Mitglied der Gruppe, ${ }^{79}$ eine Art "Super-Repräsentant" ${ }^{80}$. Gleichzeitig zeigt er sich hinsichtlich mancher Themen aufgeschlossener, besser informiert und kompetenter. ${ }^{81}$

Nicht ausschließlich mit Ähnlichkeit oder Ingroup-Orientierung zu erklären ist das Ergebnis des folgenden Versuchs: Aronson/Golden wollten aufzeigen, daß auch Aspekte, die objektiv nichts mit Glaubwürdigkeit zu tun haben, die Einschätzung der Rezipienten beeinflussen können. Die Zuweisung relevanter Glaubwürdigkeitskriterien erfolgte dabei nach bekanntem Muster: Zum Thema 'Die Wichtigkeit und Bedeutung von Arithmetik' sprachen vor insgesamt vier Schulklassen einmal ein Mann, der vorgestellt wurde als Ingenieur, der seinen Abschluß an einer Elite-Universität gemacht hat und der an einem Regierungsprojekt zur Raumfahrt mitarbeitet. Ferner wurde betont, daß er hervorragende Arbeit leistet und daß die Regierung großes Vertrauen zu ihm hat. Die zweite Quelle war ausgewiesen als Tellerwäscher, der die High School nicht beendet hat. Zusätzlich wurde als Kriterium die Hautfarbe des Sprechers variiert; sowohl der Ingenieur als auch der Tellerwäscher waren je einmal ein Farbiger und ein Weißer.

Dabei ergab sich, daß die berufliche Position der Quelle die größte Auswirkung auf die Meinungsänderung hatte, die Hautfarbe war auf den ersten Blick (in der Summe der Daten) nicht so wichtig. Als man jedoch die Rezipienten differenzierter betrachtete, zeigte sich, daß diejenigen, die in anderen, vorausgehenden Tests negative Einstellungen gegenüber Farbigen offenbart hatten, weitaus seltener ihre Meinung änderten, wenn der Sprecher ein Farbiger war. Diese vorurteilsbelasteten Personen blieben auch von den Kom-

79 Vgl. Elihu Katz: The Two Step Flow of Communication: An Up-to-date Report on an Hypothesis, in: Public Opinion Quarterly 21/1957, S. 61-78, S. 73.

80 Vgl. Joseph T. Klapper: The Effects of Mass Communication, New York 1960, S. 34.

81 Vgl. Paul F. Lazarsfeld / Herbert Menzel: Massenmedien und personaler Einfluß, in: Wilbur Schramm (Hrsg.): Grundfragen der Kommunikationsforschung, München ${ }^{3}$ 1970, S. $117-$ 139 , S. $135 \mathrm{ff}$ 
petenzmerkmalen weitgehend unbeeindruckt. ${ }^{82}$

Folgt man Simons et al., so dürften solch "irrelevante" Ähnlichkeiten oder Unähnlichkeiten keinen "instrumentellen Wert" für die Zuhörer haben, weil sie nicht zur Wahrnehmung von Kompetenz, Objektivität, Fairness etc. beitragen. ${ }^{83}$ Zudem müßten die manifesten Kompetenzhinweise die Bedeutung der Ähnlichkeit überlagern. Da dies nicht immer der Fall ist, ist davon auszugehen, daß hier emotionale Kriterien eine Rolle spielen, die man in ihrer allgemeinsten Form als 'Sympathie' bezeichnen könnte. Uneinigkeit besteht darüber, ob 'Sympathie' als Bestandteil des Konstrukts 'Glaubwürdigkeit' zu werten ist, oder ob sie eine unabhängige Komponente bei der Wahrnehmung von Personen darstellt. ${ }^{84}$ Gesichert scheint dagegen, daß einzelne Komponenten sich gegenseitig beeinflussen. So konnte etwa nachgewiesen werden, daß Prominenten, die als sympathisch eingestuft werden, auch mehr Vertrauen entgegengebracht wird. ${ }^{85}$

Ein mögliches Kriterium, erhöhte Sympathiewerte zu erlangen, ist die physische Attraktivität des Sprechers. ${ }^{86}$ Es konnte gezeigt werden, daß Personen, die

82 Elliot Aronson / Burton W. Golden: The Effect of Relevant and Irrelevant Aspects of Communicator Credibility on Opinion Change, in: Journal of Personality 30/1962, S. 135-146.

83

Vgl. Simons / Berkowitz / Moyer (1970), S. 12f. Zu den irrelevanten Kriterien ist auch das Rauchen zu rechnen: Nichtraucher sprechen Personen, die mit Zigarette abgebildet werden, geringere Glaubwürdigkeit zu, während Raucher keine Unterschiede zwischen den beiden Personengruppen machen. (Vgl. C. L. Tokheim / J. W. Wanzenried / F. C. Powell: Cigarette Smoking: Effect of Perceptions of Source Credibility, in: Psychological Reports 66/1990, S. 1388-1390.)

84 Vgl. W. Barnett Pearce: Trust in Interpersonal Communication, in: Speech Monographs 41/1974, S. 236-244, S. 239.

85 Vgl. Hershey H. Friedman / I. Friedman: Whom Do Students Trust? in: Journal of Communication 26/1976, S. 48-49; Hershey H. Friedman / Michael J. Santeramo / Anthony Traina: Correlates of Trustworthiness for Celebrities, in: Journal of Academy of Marketing Science 6/1979, S. 291-299.

86 Die Forschung zur physischen Attraktivität beschränkt sich im wesentlichen auf die Attraktivität des Gesichts. Die Operationalisierung erfolgt durch die Vorlage von Photos. Die 
als attraktiv gelten, gleichzeitig bessere Charaktereigenschaften zugeschrieben werden: "A consistent pattern emerges, that of the unattractive person being associated with the negative or undesirable pole of the adjective scales and the highly attractive person being judged significantly more positively." ${ }^{87}$ Ferner gibt es Hinweise, daß attraktive Personen als eigenständig und weniger beeinflußbar betrachtet werden. ${ }^{88}$ Im Hinblick auf die Glaubwürdigkeit zieht Joseph daraus die Schlußfolgerung:

Festlegung, wer als attraktiv gilt, geht dabei in der Regel zurück auf die Summe der Einschätzungen verschiedener "Juroren" in Pretests ("truth by consensus"). Dabei wird lediglich der Gesamteindruck berücksichtigt. Es erfolgt keine Variation einzelner Merkmale. (Vgl. dazu W. Benoy Joseph: The Credibility of Physically Attractive Communicators: A Review, in: Journal of Advertising 11/1982, S. 15-24, S. 15f.) Zur Kritik an dieser Methode vgl. Manfred Hassebrauck: Die Beurteilung der physischen Attraktivität: Konsens unter Urteilern? In: Zeitschrift für Sozialpsychologie 14/1983, S. 152-161. Zur Bedeutsamkeit einzelner Merkmale siehe: Manfred Hassebrauck / Reiner Niketta (Hg.): Physische Attraktivität, Göttingen u.a. 1993, darin insbesondere: Martin Schuster: Gesichtsschönheit: Begriff, Geschichte und Merkmale, S. 11-28; Manfred Hassebrauck: Die Beurteilung der physischen Attraktivität, S. 29-59; Reiner Niketta: Das Stereotyp der physischen Attraktivität, S. 163-200. Zum Zusammenhang von Sympathie und Attraktivität siehe auch: Harold Sigall / Elliot Aronson: Liking for an Evaluator as a Function of Her Physical Attractiveness and Nature of the Evaluations, in: Journal of Experimental Social Psychology 5/1969, S. 93-100.

87 A. G. Miller: Role of Physical Attractiveness in Impression Formation, in: Psychonomic Science 19/1970, S. 241-243, S. 242. Vgl. dazu auch: Karen K. Dion / Ellen Berscheid / Elaine Walster: What Is Beautiful Is Good, in: Journal of Personality and Social Psychology 24/1972, S. 285-290; Ellen Berscheid / Elaine Walster: Physical Attractiveness, in: Leonard Berkowitz (Hrsg.): Advances in Experimental Social Psychology, Vol. 7, New York/London 1974, S. 157215, S. 168ff.; Kenneth L. Dion / Karen Dion: Belief in a Just World and Physical Attractiveness Stereotyping, in: Journal of Personality and Social Psychology 52/1987, S. 775-780; Barry Gillen: Physical Attractiveness: A Determinant of Two Types of Goodness, in: Personality and Social Psychology Bulletin 7/1981, S. 277-281; Manfred Hassebrauck: Der Einfluß von Attitüdenähnlichkeit und physischer Attraktivität auf die Beurteilung von Personen, in: Psychologische Beiträge 27/1986, S. 265-276; Gordon L. Patzer: The Physical Attractiveness Phenomena, New York 1985; Michael J. Baker / Gilbert A. Churchill, Jr.: The Impact of Physically Attractive Models on Advertising Research, in: Journal of Marketing Research 14/1977, S. 538-555.

88 Vgl. A. G. Miller: Social Perception of Internal-External Control, in: Perceptual and Motor Skills 30/1970, S. 103-110. 
"If physically attractive sources are perceived to have an internal locus of control, they may also be perceived as individuals who are not easily influenced or manipulated by others, and whose opinions spring from independent thinking and personal convictions, all of these being qualities which denote greater source credibility." 89

Nicht nur die Zuschreibung von Eigenschaften, sondern auch die Einschätzung von Fähigkeiten kann von Attraktivität beeinflußt werden: So konnten Landy/Sigall nachweisen, daß ein eher schlechter Essay besser beurteilt wurde, wenn eine attraktive Studentin als Quelle benannt wird, wohingegen bei einem qualitativ guten Essay die Variable 'Attraktivität' nicht zu einer unterschiedlichen Einschätzung führte. ${ }^{90}$ Ferner kann Attraktivität die Wahrnehmung der Sprechkompetenz beeinflussen: Attraktiven Sprechern wird ein flüssigerer Vortragsstil bescheinigt. ${ }^{91}$ Wenn, wie diese Befunde andeuten,

$89 \quad$ Joseph (1982), S. 16.

90 Vgl. David Landy / Harold Sigall: Beauty is Talent: Task Evaluation as a Function of the Performer's Physical Attractiveness, in: Journal of Personality and Social Psychology 29/1974, S. 299-304. Ein vergleichbarer Befund ergibt sich bei: Robert M. Kaplan: Is Beauty Talent? Sex Interaction in the Attractiveness Halo Effect, in: Sex Roles 4/1978, S. 195-204. Der Effekt stellte sich hier allerdings nur ein, wenn männliche Versuchspersonen weibliche Quellen beurteilten. Bei männlichen Quellen ergaben sich keine signifikanten Unterschiede und das Geschlecht der Beurteiler spielte keine Rolle. Daß für männliche Versuchspersonen Attraktivität eine größere Rolle spielt als für weibliche zeigen auch Wolfgang Stroebe et al:: Effects of Physical Attractiveness, Attitude Similarity, and Sex on Various Aspects of Interpersonal Attraction, in: Journal of Personality and Social Psychology 18/1971, S. 79-91 sowie Peter Borkenau: Reicher Mann und schöne Frau? Zwei Studien zu Geschlechtsunterschieden in der Partnerpräferenz, in: Zeitschrift für Sozialpsychologie 24/1993, S. 289-297. Zum Teil werden die Ergebnisse von Landy/Sigall auch bestätigt bei: Carole Kovalic Holahan / Cookie White Stephan: When Beauty Isn't Talent: The Influence of Physical Attractiveness, Attitudes Toward Women, and Competence on Impression Formation, in: Sex Roles 7/1981, S. 867-876. Die Forscherinnen zeigen zusätzlich auf, daß die Einschätzungen bezüglich Talent und Kompetenz nicht nur in Abhängigkeit von der Attraktivität der weiblichen Quelle stehen, sondern variieren, je nachdem, ob die Versuchspersonen eher 'traditionelle' oder 'liberale' Einstellungen gegenüber Frauen haben. Den Befunden von Landy/Sigall widersprechen neuere Befunde von Manfred Schmitt: Schönheit und Talent: Untersuchungen zum Verschwinden des Halo-Effekts, in: Zeitschrift für Experimentelle und Angewandte Psychologie 39/1992, S. 475-492.

91 Vgl. Shelly Chaiken: Communicator Physical Attractiveness and Persuasion, in: Journal of Personality and Social Psychology 37/1979, S. 1387-1397. 
attraktive Personen als kompetenter wahrgenommen werden, ist zumindest ein mittelbarer Einfluß der Attraktivität auf die Glaubwürdigkeit einer Quelle naheliegend, bisher allerdings nicht eindeutig nachgewiesen.

Neben der Zuschreibung von Eigenschaften und Fähigkeiten wurde auch geprüft, ob Meinungsänderungen erreicht werden. Haiman führte zur Überprüfung der Frage, ob Sympathie und Attraktivität Einfluß auf die Meinungsänderung haben, folgendes Experiment durch: Zum Thema "Gesetzliche Krankenversicherung" versucht der gleiche Sprecher auf unterschiedliche Publika jeweils einmal einen "guten" und einen "schlechten" Eindruck zu machen, wobei lediglich die Manipulationen zur Erzielung des "schlechten" Eindrucks näher beschrieben werden: Der Sprecher tritt unrasiert, ungekämmt und mit einer unvorteilhaften Brille auf. Dazu trägt er ein leicht verschmutztes Hemd, das am Ärmel leicht zerrissen ist, eine schlecht gebundene Krawatte, schmutzige, ungebügelte Hosen und ungeputzte Schuhe. Zudem verhält er sich unfreundlich, überheblich und sarkastisch.

Daß die Manipulation erfolgreich war, zeigt sich in signifikant unterschiedlichen Werten für Sympathie und Attraktivität. Dagegen zeigen sich keine signifikanten Unterschiede bei der Einschätzung von Kompetenz, Aufrichtigkeit und Dünkelhaftigkeit der Quelle. Und obwohl sich tendenzielle Unterschiede hinsichtlich der Meinungsänderung auf seiten der Zuhörer zeigen, sind auch diese nicht signifikant. ${ }^{92}$ Mills und Aronson berichten von einem schwachen Zusammenhang zwischen Attraktivität und Meinungsänderung. ${ }^{93}$ Hingegen ergibt sich bei Snyder und Rothbart eine deutlich stärkere Meinungsänderung für den attraktiven Kommunikator. Auch hier zeigen sich jedoch

92 Vgl. Franklyn S. Haiman: An Experimental Study of the Effects of Ethos in Public Speaking, in: Speech Monographs 16/1949, S. 190-202, S. 197f. Kein Einfluß der Attraktivität auf Meinungsänderungen ergab sich auch bei James E. Maddux / Ronald W. Rogers: Effects of Source Expertness, Physical Attractiveness, and Supporting Arguments on Persuasion: A Case of Brains over Beauty, in: Journal of Personality and Social Psychology 39/1980, S. 235-244.

93 Vgl. Judson Mills / Elliot Aronson: Opinion Change as a Function of the Communicator's Attractiveness and Desire to Influence, in: Journal of Personality and Social Psychology 1/1965, S. 173-177. Glaubwürdigkeitsmerkmale wurden hier nicht gemessen. 
zwischen attraktiven und unattraktiven Quellen keine unterschiedlichen Wahrnehmungen hinsichtlich Glaubwürdigkeit und Kompetenz. Die Meinungsänderung wird ausschließlich darauf zurückgeführt, daß die attraktive Quelle sympathischer ist. ${ }^{94}$ Diese Unabhängigkeit der Dimensionen 'Attraktivität' und 'Kompetenz' wird auch von anderer Seite bestätigt. ${ }^{95}$ Im Widerspruch dazu fand Patzer, daß mit steigender Attraktivität sowohl die Sympathie für die Quelle als auch ihre wahrgenommene Kompetenz und Vertrauenswürdigkeit zunahm. ${ }^{96}$ Bei diesem Experiment, dessen Stimulusmaterial eine Anzeige für ein Schmerzmittel war, wurde allerdings nicht in Betracht gezogen, daß sowohl Quelle als auch Versuchspersonen Studenten waren. Mithin können die Glaubwürdigkeitseffekte auch auf die Ähnlichkeit hinsichtlich der Gruppenzugehörigkeit zurückzuführen sein.

Für McCroskey/McCain ist die körperliche Attraktivität nur ein Faktor im Rahmen der interpersonellen Anziehungskraft. Daneben machen sie noch zwei weitere Faktoren aus: "Social Attraction" steht für den Wunsch, mit der zu beurteilenden Person befreundet zu sein und "Task Attraction" umschreibt die Eignung der zu beurteilenden Person für die Lösung einer gemeinsamen

94 Vgl. Mark Snyder / Myron Rothbart: Communicator Attractiveness and Opinion Change, in: Canadian Journal of Behavioral Science 3/1971, S. 377-387, S. 385.

95 Vgl. Joann Horai / Nicholas Naccari / Elliot Fatoullah: The Effects of Expertise and Physical Attractiveness upon Opinion Agreement and Liking, in: Sociometry 37/1974, S. 601606; Shelly Chaiken: Communicator Physical Attractiveness and Persuasion, in: Journal of Personality and Social Psychology 37/1979, S. 1387-1397.

96 Vgl. Gordon L. Patzer: Source Credibility as a Function of Communicator Physical Attractiveness, in: Journal of Business Research 11/1983, S. 229-241. Ein positiver Zusammenhang von Glaubwürdigkeit und Aussehen - speziell Bekleidung - wird auch hergestellt von Gwendolyn S. O'Neal / Mary Lapitsky: Effects of Clothing as Nonverbal Communication on Credibility of the Message Source, in: Clothing and Textiles Research Journal 9/1991, S. 28-34. Auch hier diente Anzeigenwerbung als Stimulusmaterial. Harris et al. finden, daß Frauen, die im formellen Kostüm auftreten (im Gegensatz zu einem lässigeren Rock oder Hosen) unter anderen als erfolgreicher, attraktiver und intelligenter eingestuft werden. (Vgl. Mary B. Harris et al.: Clothing: Communication, Compliance, and Choice, in: Journal of Applied Social Psychology 13/1983, S. 88-97.) 
Aufgabe. ${ }^{97}$ Soweit man Attraktivität in Zusammenhang mit Glaubwürdigkeit bringt, ist es aber nicht nötig, diesen beiden Faktoren zusätzliche Aufmerksamkeit zu widmen, da sie nahe bei den Dimensionen 'Akzeptanz' und 'Kompetenz' angesiedelt sind.

Das gute Aussehen spielt also eine Rolle bei der Einschätzung verschiedener Eigenschaften und Fähigkeiten einer Quelle und kann Einfluß auf die Meinungsänderung haben. Die Behauptung: "Physically attractive persons are more credible than less physically attractive ones" ${ }^{18}$ wird in dieser Ausschließlichkeit von den vorliegenden Studien jedoch keinesfalls gestützt. In den Fällen, in denen geprüft wurde, ob Attraktivität die Einschätzung der Kompetenz beeinflußt, konnten kaum Zusammenhänge nachgewiesen werden. Eine Ausnahme stellt, unter bestimmten Bedingungen, die Werbung dar. Wenn die Attraktivität eines Werbenden in Zusammenhang mit dem Produkt steht, wächst die Glaubwürdigkeit der Anzeige signifikant. ${ }^{99}$

97 Vgl. James C. McCroskey / Thomas A. McCain: The Measurement of Interpersonal Attraction, in: Speech Monographs 41/1974, S. 261-266. Vgl. dazu auch: Wolfgang Stroebe et al.: Effects of Physical Attractiveness, Attitude Similarity, and Sex on Various Aspects of Interpersonal Attraction, in: Journal of Personality and Social Psychology 18/1971, S. 79-91 sowie allgemein: Theodore M. Newcomb: The Prediction of Interpersonal Attraction, in: Carl W. Backman / Paul F. Secord (Hg.): Problems in Social Psychology. Selected Readings, New York u.a. 1966, S. 168-179.

98 Richard D. Rieke / Malcolm O. Sillars: Argumentation and the Decision Making Process, New York u.a. 1975, S. 155.

99 Dies gilt nicht zuletzt für prominente Personen. Vgl. dazu Michael A. Kamins: An Investigation into the "Match-Up" Hypothesis in Celebrity Advertising: When Beauty May be Only Skin Deep, in: Journal of Advertising 19/1990, S. 4-13 und Roobina Ohanian: The Impact of Celebrity Spokespersons' Perceived Image on Consumers' Intention to Purchase, in: Journal of Advertising Research 31/1991, S. 46-54. Die Verwendung prominenter Fürsprecher ist jedoch anderen Werbeformen nicht von vorneherein überlegen. Als gleichermaßen glaubwürdig erweist sich die sogenannte "word-of-mouth- communication", die Ingroup-Orientierung simuliert, indem sie eine Unterhaltung zwischen typischen Produktverwendern präsentiert. Ein Werbespot dieser Art wird als vertrauenswürdig wahrgenommen und spiegelt Kompetenz (durch Erfahrung) wider. Der prominente Sprecher hat dagegen stärkere Werte in den Dimensionen 'Dynamik' und 'Attraktivität'. (Vgl. Patrick M. Sobczak / Michael R. Bowers: An Experimental Design for Measuring the Effects of Source Credibility and Goal Behavior Estimate in Health Promotion Television Commercials, in: Psychology. A Journal of Human Behavior 30/1993, S. 30-43.) Zur 
Joseph konnte darüberhinaus zeigen, daß die Einschätzung der Vertrauenswürdigkeit ebenfalls unabhängig vom Aussehen der Quelle war. Hingegen ergab sich ein Zusammenhang mit der Komponente 'Dynamik': "This finding was interpreted as being an indicator of the greater arousal or attention value that attractive communicators possess when compared to their less attractive counter parts." ${ }^{100}$ Ferner gibt es Anzeichen dafür, daß das Merkmal der Attraktivität als 'Ersatzkriterium' herangezogen wird, wenn konkrete Hinweise (etwa auf die Kompetenz der Quelle) fehlen:

"When the source was expert, her physical attractiveness made little difference in terms of subject's preferences; however when she was inexpert, subjects agreed more with the high attractive source than with the medium or low attractive source. Apparently, when objective or task-related source characteristics (e.g., expertise) are weak, subjects resort to 'irrelevant' cues (such as physical attractiveness) to form opinions." ${ }^{101}$

Diese Einschätzung wird gestützt durch den Befund, daß Attraktivität zwar in Verbindung mit emotional getönten, nicht aber bei eher rationalen Botschaften Einfluß auf Einstellungen hat. ${ }^{102}$

\subsection{Sprechverhalten}

eingeschränkten Brauchbarkeit von Prominenten für die Werbung im Hinblick auf ihre Glaubwürdigkeit vgl. auch Michael A. Kamins et al.: Two-Sided Versus One-Sided Celebrity Endorsements: The Impact on Advertising Effectiveness and Credibility, in: Journal of Advertising 18/1989, S. 4-10.

100 Joseph (1982), S. 19. Joseph beruft sich hier auf seine (unveröffentlichte) Dissertation: W. Benoy Joseph: Effect of Communicator Physical Attractiveness and Expertness on Opinion Change and Information Processing, Columbus: Ohio State University 1977.

$101 \quad$ Ebd.

102 Vgl. Suzanne R. Pallak / Enid Murroni / Juliann Koch: Communicator Attractiveness and Expertise, Emotional versus Rational Appeals, and Persuasion: A Heuristic versus Systematic Processing Interpretation, in: Social Cognition 2/1983, S. 122-141. 
"While considerable emphasis has been placed on the 'who the source is' dimension, little consideration has been given to 'how the source says it"'. ${ }^{103}$ Damit ist eine weitere Möglichkeit, die Glaubwürdigkeit der Quelle zu manipulieren, umrissen, die darin liegt, gezielte Variationen des Sprechverhaltens vorzunehmen. Im Blickpunkt stehen hier vor allem die Elemente, die im Bereich der verhaltensorientierten Glaubwürdigkeitsforschung als extralinguistisch bezeichnet werden, also Merkmale wie Sprechgeschwindigkeit, Stimmhöhe, Verzögerungen durch Pausen, Sprechstörungen wie grammatikalische Fehler, überflüssige Wiederholungen von Wörtern oder Satzteilen, unvollständige Sätze, Stottern und Versprecher. Die nonverbalen Elemente des Sprechverhaltens erfahren weniger Aufmerksamkeit. ${ }^{104}$

Während die verhaltensorientierte Glaubwürdigkeitsforschung sich vornehmlich darauf konzentriert, Indizien für Täuschungen zu finden (vgl. Kap. II. A), sollen hier Untersuchungen betrachtet werden, die das extralinguistische Verhalten in Zusammenhang bringen mit Einstellungsänderungen einerseits und den Dimensionen der Glaubwürdigkeit andererseits. Obgleich bei diesen extralinguistischen Elementen nicht gesichert ist, inwieweit sie tatsächlich Hinweise darauf geben, wann ein Sprecher die Unwahrheit sagt, kann gezeigt werden, daß sie in der Wahrnehmung der Rezipienten zu Abstrichen bezüglich der Glaubwürdigkeit führen können:

"As a source of information on which to base reliable psychological diagnoses, vocal cues are of limited value. But inferences based on these cues are of interest in the study of communication regardless of their accuracy. To the extent that a speaker is perceived as having high or low

103 Gerald R. Miller / Murray A. Hewgill: The Effect of Variations in Nonfluency on Audience Ratings of Source Credibility, in: Quarterly Journal of Speech 50/1964, S. 36-44, S. 36.

104 Die begriffliche Trennung, die die verhaltensorientierte Glaubwürdigkeitsforschung vornimmt, wird hier beibehalten, obgleich einzelne Autoren alle Variablen des Sprechverhaltens als 'nonverbal' bezeichnen. Vgl. z.B.: Judee K. Burgoon / Thomas Birk / Michael Pfau: Nonverbal Behaviors, Persuasion, and Credibility, in: Human Communication Research 17/1990, S. 140-169. 
credibility and desirable or undesirable personality characteristics on the basis of his delivery style, vocalic communication may affect the continuation of an interaction or the effectiveness of a message." 105

Diese extralinguistischen Elemente werden häufig ebenfalls mit dem Etikett 'Dynamik' bedacht, sind jedoch nicht identisch mit dem von Berlo et al. ermittelten Faktor gleichen Namens, wenngleich der nachfolgende Überblick über einzelne Befunde zeigt, daß in der Einschätzung von Rezipienten durchaus ein Zusammenhang besteht. Die Vorgehensweise der Untersuchungen gleicht sich im wesentlichen: Die Probanden werden mit Tonbandaufnahmen konfrontiert, die hinsichtlich der Sprechervariablen manipuliert sind. Rückschlüsse auf die Glaubwürdigkeit werden mittels anschließend vorgelegter Semantischer Differentiale gezogen, wobei sich die meisten Forscher einer verkürzten Fassung der Skala von Berlo et al. bedienen, mithin also die Komponenten 'Kompetenz', 'Vertrauenswürdigkeit' und 'Dynamik' berücksichtigen. Soweit Meinungs- und Einstellungsänderungen erhoben werden, wird die im Rahmen der Einstellungsforschung übliche Vorher-NachherMessung bezüglich des jeweiligen Themas durchgeführt.

Die Prüfung des Einflusses sogenannter 'Nonfluencies' (Störungen im Sprechfluß, speziell Stottern, Versprecher, Wiederholungen und stimmhafte Pausen wie 'äh') ergab, daß die wahrgenommene Kompetenz des Sprechers abnahm, wenn die Anzahl der Störungen zunahm. Sprecher, die einen fehlerlosen Vortrag hielten, wurden zudem als dynamischer eingestuft; es ergab sich aber kein Einfluß auf die Einschätzung der Dimension 'Vertrauenswürdigkeit' sowie auf Einstellungsänderungen. ${ }^{106}$ Hingegen ermittelten McCros-

105 W. Barnett Pearce / Bernard J. Brommel: Vocalic Communication in Persuasion, in: Quarterly Journal of Speech 58/1972, S. 298-306, S. 299.

106 Vgl. Miller / Hewgill (1964) sowie Kenneth K. Sereno / Gary J. Hawkins: The Effects of Variations in Speaker's Nonfluency upon Audience Ratings Toward the Speech Topic and Speaker's Credibility, in: Speech Monographs 34/1967, S. 58-64. Siehe ergänzend auch C. H. Lay / B. F. Burron: Perception of the Personality of the Hesitant Speaker, in: Perceptual and Motor Skills 26/1968, S. 951-956. 
key/Mehrley Auswirkungen sowohl auf alle drei Glaubwürdigkeitsdimensionen als auch auf Einstellungsänderungen. ${ }^{107}$ Burgoon et al. legten leicht abweichende Glaubwürdigkeitsdimensionen zugrunde und fanden Einflüsse auf 'Kompetenz', 'Charakter' (vergleichbar mit Teilaspekten der 'Vertrauenswürdigkeit') und 'Umgänglichkeit' (die eine Ausprägung des Faktors 'Sympathie' darstellt). ${ }^{108}$

Addington betrachtete neben der Stimmodulation und Sprechgeschwindigkeit die Faktoren Stimmqualität und Artikulation und fand, daß die drei Komponenten 'Kompetenz', 'Vertrauenswürdigkeit' und 'Dynamik' vom Sprechstil beeinflußt wurden, wobei die Auswirkungen auf die Wahrnehmung der Kompetenz am größten waren. Vornehmlich fehlerhafte Artikulation, eine monotone Tonlage und unangenehme Stimmqualitäten (wie nasales Sprechen oder Heiserkeit) führten zu einer Abnahme der Glaubwürdigkeit. ${ }^{109}$

Die Einschätzung der Bedeutung einzelner Faktoren kann dadurch erschwert werden, daß mehrere Elemente gebündelt statt einzeln variiert werden. Beispielsweise vergleichen Pearce/Conklin einen "conversational style", der sich durch niedrige Tonhöhe, langsames und leises Sprechen sowie geringe Stimmodulation auszeichnet mit einem "dynamic style" (lebhafter, variantenreicher, höhere Tonhöhe, schneller). Die Vortragsweisen stellen eher unterschiedliche, individuell gefärbte Ausdrucksmöglichkeiten eines "guten" Sprechers dar; entsprechend gibt es keine Unterschiede hinsichtlich der

Vgl. James C. McCroskey / R. Samuel Mehrley: The Effects of Disorganization and Nonfluency on Attitude Change and Source Credibility, in: Speech Monographs 36/1969, S. 13-21. Zusätzlich wurde hier noch eine inhaltliche Variable geprüft: der Einfluß einer unlogischen Struktur bei der Aufeinanderfolge von Argumenten. Diese "desorganisierte" Version erbrachte lediglich Abstriche hinsichtlich der Kompetenz des Sprechers. Ein vergleichbares Ergebnis berichten auch Harry Sharp, Jr. / Thomas McClung: Effects of Organization on the Speaker's Ethos, in: Speech Monographs 33/1966, S. 182-183. 
Einschätzung der Kompetenz. ${ }^{110}$ Der "conversational style" wurde von den Zuhörern aber als weniger dynamisch, vertrauenswürdiger, ehrlicher, gütiger, wärmer, freundlicher und angenehmer empfunden. ${ }^{111}$ Neben den Dimensionen 'Kompetenz', 'Vertrauenswürdigkeit' und 'Dynamik' spielt hier also auch die Dimension 'Sympathie' (von den Forschern als 'Evaluation' bezeichnet) eine Rolle; allerdings erbrachten die beiden Sprechstile keine Unterschiede hinsichtlich der Änderung von Einstellungen. ${ }^{112}$

Sofern die einzelnen Variablen keiner technischen Manipulation unterliegen, besteht der Vorteil der kombinierten Betrachtung allerdings darin, daß die Vortragsweise natürlicher erscheint, zumal Zuhörer einzelne stimmliche Attribute nicht unabhängig voneinander wahrnehmen: ${ }^{113}$

"Given that many nonverbal cues may not be particularly meaningful or potent until combined into sets of related behaviors, past research of isolated cues may have underestimated the effect of nonverbal behavior in persuasive contexts." 114

$110 \quad$ Vgl. Pearce / Brommel (1972), S. 303.

111 Vgl. W. Barnett Pearce / Forrest Conklin: Nonverbal Vocalic Communication and Perceptions of a Speaker, in: Speech Monographs 38/1971, S. 235-241. Einer der beteiligten Forscher gibt allerdings an anderer Stelle zu bedenken, daß die Validität fraglich ist und die Ergebnisse möglicherweise ein Artefakt darstellen, da die verwendeten Stimmen elektronisch gefiltert wurden, um unerwünschte Elemente auszuschließen und daher unter Umständen unnatürlich klangen. (Vgl. Pearce / Brommel (1972), S. 299f.) Partiellen Widerspruch erfahren die Befunde durch Brown/Strong/Rencher: Hier wirkt sich zwar auch die niedrige Tonhöhe aber eine größere Variation bei der Intonation positiv auf die Glaubwürdigkeit aus. (Vgl. Bruce L. Brown / William J. Strong / Alvin C. Rencher: Perceptions of Personality from Speech: Effects of Manipulations of Acoustical Parameters, in: Journal of the Acoustical Society of America 54/1973, S. 29-35 sowie dies.: Fifty-four Voices from Two: The Effects of Simultaneous Manipulations of Rate, Mean Fundamental Frequency and Variance of Fundamental Frequency on Ratings of Personality from Speech, in: Journal of the Acoustical Society of America 55/1974, S. 313-318.) Credibility, in: Journalism Quarterly 55/1978, S. 276-281, 300, S. 280. 
Eine umfangreiche Studie von Burgoon et al., die alle nonverbalen und extralinguistischen Variablen einer Prüfung unterzieht, bestätigt nur einen Teil der vorliegenden Ergebnisse. Demnach wirken sich ein flüssiger Vortragsstil und "Unmittelbarkeit" (gekennzeichnet durch häufigen Blickkontakt und Lächeln) besonders positiv auf Glaubwürdigkeit und Einstellungsänderungen aus, während die stimmlichen Elemente (Tempo, Lautstärke, Stimmhöhe) ebensowenig eine Rolle spielen wie Selbst- und Objektmanipulationen. ${ }^{115}$

Widersprüchliche Befunde in diesem Forschungsbereich haben ihre Ursache in erster Linie in unterschiedlichen Operationalisierungen. So ist etwa nicht klar, was einzelne Forscher unter "schnellem" oder "langsamen" Tempo, "lauter" oder "leiser" Stimme verstehen, da keine objektiven Meßlatten angelegt werden, sondern eher subjektiv Abweichungen von "normalem" Sprechverhalten konstatiert werden. Zusätzliche Probleme hinsichtlich der Vergleichbarkeit ergeben sich, wenn für die Bestimmung der Glaubwürdigkeit unterschiedliche Dimensionen verwendet werden. ${ }^{116}$

Trotz dieser Vorbehalte ist davon auszugehen, daß einige Zusammenhänge als gesichert gelten können. So etwa die Auswirkungen von Sprechstörungen und Stokungen im Redeverlauf, die als mangelnde sprachliche Kompetenz zu deuten sind und auf die Einschätzungen der Fachkompetenz abfärben. Eine Studie berichtet, daß Rezipienten sich bei der Frage nach der Glaubwürdigkeit ausdrücklich auch an Indizien mangelnder Sprachbeherrschung orientieren. ${ }^{117}$ Ähnliches gilt für die Sprechgeschwindigkeit: "Wer schneller spricht, wird im allgemeinen für kompetenter und intelligenter gehalten als jemand, der die

\footnotetext{
115 Vgl. Burgoon / Birk / Pfau (1990), S. 157ff.

116 Vgl. dazu auch Burgoon / Birk / Pfau (1990), S. 145.

117 Vgl. Schweitzer / Ginsburg (1966), S. 96. Der Zusammenhang deutet sich auch an bei: Anthony Mulac / A. Robert Sherman: Relationships among Four Parameters of Speaker Evaluation: Speech Skill, Source Credibility, Subjective Speech Anxiety, and Behavioral Speech Anxiety, in: Speech Monographs 42/1975, S. 302-310.
} 
gleiche Information in geringerer Geschwindigkeit kommuniziert."118 Variablen, die Stimmeigenschaften und Vortragsweisen sowie nonverbale Elemente wie Blickkontakt und Lächeln umfassen, scheinen bevorzugt, wenn auch nicht ausschließlich, Einfluß auf die Dimensionen 'Vertrauenswürdigkeit' und 'Sympathie' zu haben. Daß diese Faktoren auch im Bewußtsein der Rezipienten eng mit Glaubwürdigkeit verknüpft sind, bestätigt eine Faktorenanalyse freier Antworten: Neben den bekannten Dimensionen ergibt sich auch ein Faktor 'Artikulation'. ${ }^{119}$

\subsection{Zusätzliche Einflußfaktoren}

\subsubsection{Das Geschlecht des Sprechers}

Ein weiterer potentieller Einflußfaktor, der zu den "irrelevant cues" im Hinblick auf die Glaubwürdigkeit gehört, ist das Geschlecht des Sprechers. Rieke und Sillars konstatieren in ihrem Überblick zur Glaubwürdigkeitsforschung: "Men are generally perceived to be more credible than women, although a physically and mentally attractive woman is more credible than a less attractive man." ${ }^{120}$

Betrachtet man die zugrundeliegenden Studien, auf die sie sich beziehen, so ist allerdings fraglich, ob die Behauptung in dieser allgemeinen Form aufrechterhalten werden kann. Die erste der Untersuchungen, die in diesem Zusammenhang zur Diskussion gestellt wird, beschäftigt sich nicht mit Glaubwürdigkeit im engeren Sinne. Vielmehr wird nachgewiesen, daß bei einer autokinetischen Versuchsanordnung, bei der es um die Einschätzung von Grö-

\footnotetext{
118 Zu diesem Schluß gelangt Köhnken (1990: 54) unter Berufung auf die Studien von N. Miller et al.: Speed of Speech and Persuasion, in: Journal of Personality and Social Psychology 34/1976, S. 615-625 und B. L. Smith et al.: Effects of Speech Rate on Personality Perception, in: Language and Speech 18/1975, S. 145-152.

$119 \quad$ Vgl. Singletary (1976), S. 318.

$120 \quad$ Rieke / Sillars (1975), S. 155.
} 
ßenverhältnissen geht, Frauen generell eher bereit sind, bei einer "Gegenstimme" von ihrer Einschätzung abzuweichen und daß männliche "Gegenstimmen" auf beide Geschlechter größere Beeinflussungskraft haben als weibliche. ${ }^{121}$ Daraus die Folgerung abzuleiten, daß männliche Quellen generell überzeugender sind als weibliche - und zwar unabhängig vom verwendeten Medium und vom angesprochen Thema ${ }^{122}$ - erscheint allerdings sehr gewagt. Wenngleich nicht ausgeschlossen werden kann, daß hier "culturally determined personality characteristics" ihren Niederschlag finden ${ }^{123}$, ist doch einschränkend anzumerken: 'Persuasion' fand hier im Grunde nicht statt, da die Entscheidungen nicht mit Argumenten untermauert wurden (soweit aus der Darstellung ersichtlich fand zwischen den Probanden keine Kommunikation statt). Zudem kann sich die Art der gestellten Aufgabe als bedeutsam erweisen. So ist vorstellbar, daß Männern implizit eine größere Kompetenz zugeschrieben wird, wenn es um das Einschätzen von Größen- und Längenmaßen geht abgeleitet von der Vorstellung, daß Männer über ausgeprägtere Fähigkeiten im Bereich Mathematik verfügen. Entsprechend wäre zu prüfen, ob sich an den Befunden etwas ändert, wenn die Aufgabenstellung eher "weibliche" Kompetenz verlangt. (Denkbar wäre hier etwa eine Schätzung des Alters von Kleinkindern anhand von Photos, wenn man im Bereich gängiger Stereotype bleiben will.)

In einer häufig als Beleg zitierten weiteren Untersuchung setzt sich derselbe Verfasser speziell mit der Frage der Glaubwürdigkeit auseinander. ${ }^{124}$ Ein Text über die Effektivität der Vereinten Nationen wird jeweils von einem männlichen und einem weiblichen Radiosprecher präsentiert oder in Form eines

\footnotetext{
121 Vgl. James O. Whittaker: Sex Differences and Susceptibility to Interpersonal Persuasion, in: Journal of Social Psychology 66/1965, S. 91-94.

$122 \quad$ Vgl. ebd. S. 93.

123 Ebd.

124 Vgl. James O. Whittaker / Robert D. Meade: Sex of the Communicator as a Variable in Source Credibility, in: Journal of Social Psychology 72/1967, S. 27-34.
} 
Leitartikels mit männlichem oder weiblichem Verfassernamen vorgelegt. Mittels verschiedener Skalen werden dann Glaubwürdigkeit und Meinungsänderung abgefragt. Die Untersuchung wird in fünf Ländern durchgeführt.

Ein Blick auf die Ergebnisse zeigt, daß die verallgemeinernde Schlußfolgerung von Rieke / Sillars ${ }^{125}$ durch die Studie nicht gedeckt ist; die Verfasser selbst interpretieren differenzierter: Zunächst können sie Unterschiede hinsichtlich gesprochener und geschriebener Kommunikation aufzeigen. Die Tonbandversion erwies sich generell (unabhängig vom Geschlecht) als geeigneter, um Meinungsänderungen herbeizuführen; in drei der fünf Länder wurden dabei die männlichen Quellen signifikant glaubwürdiger wahrgenommen als die weiblichen. Bei der schriftlichen Präsentation wurde lediglich in einem Land eine signifikant höhere Glaubwürdigkeit für den männlichen Verfasser beobachtet. Anders als in der ersten Studie wird hier ausdrücklich eine Einschränkung bezüglich des Themas vorgenommen: "When political or quasipolitical information is presented by a male, it apparently tends to be much more readily accepted." ${ }^{126}$ Zieht man zusätzlich in Betracht, daß es sich bei den untersuchten Ländern (Brasilien, Hongkong, Indien, Rhodesien und Jordanien) um solche handelt, in denen Frauen in der öffentlichen Kommunikation eine untergeordnete Bedeutung zukommt, so erscheint es fast schon verwunderlich, daß die Ergebnisse nicht eindeutiger ausfallen. Jedenfalls aber liefern sie keine ausreichende Grundlage, um allgemeine Aussagen bezüglich der Glaubwürdigkeit von Männern und Frauen zu treffen.

Eine eindeutige Bestätigung der These findet sich bei Miller/McReynolds: Sie legten ihren Probanden eine Pressemitteilung zum Thema "Entwicklung eines ABM-Missile-Systems" vor. Die Quelle wurde beschrieben als Doktor der Kernphysik, Direktor(in) eines Labors für Radiologie und wissenschaftliche(r) Berater(in) des National Security Council. Variiert wurde lediglich der Vorname (Dr. Robert Stapleton vs. Dr. Gretchen Stapleton). Ferner wurde im

125 Vgl. Rieke / Sillars (1975), S. 146f., S. 155.

$126 \quad$ Whittaker / Meade (1967), S. 31. 
Text jeweils zweimal das geschlechtsspezifische Personalpronomen verwendet. Die Kompetenzeinschätzung für die männliche Quelle lag signifikant höher, wobei der Effekt bei weiblichen Rezipienten sogar noch deutlicher ausfiel. Eine mögliche Erklärung sehen die Forscher darin, daß Physik als eher "männliche" Wissenschaft aufgefaßt wird. ${ }^{127}$ Eine weitere Untersuchung erbringt keine signifikanten Unterschiede zwischen männlichen und weiblichen Professoren hinsichtlich Kompetenz und Vertrauenswürdigkeit, stellt aber fest, daß die männlichen Quellen als dynamischer eingestuft wurden. ${ }^{128}$

Zur Erklärung der unterschiedlichen Glaubwürdigkeit der Geschlechter werden in der gesprochenen Kommunikation schließlich die unterschiedlichen Sprechstile von Männern und Frauen herangezogen. Demnach ist es "typisch weiblich", abschwächende Formulierungen zu wählen ("ich denke", "ich glaube, daß...") sowie zögernder und stockender zu sprechen. Werden solche sprachlichen Eigenheiten vermieden, steigt -unabhängig vom Geschlecht - die Glaubwürdigkeit. ${ }^{129}$ Andere sprachliche Besonderheiten, die eher Frauen zugeschrieben werden (korrekte Aussprache, keine Unterbrechungen, Tonhöhe

127 Vgl. Gerald R. Miller / Michael McReynolds: Male Chauvinism and Source Competence: A Research Note, in: Speech Monographs 40/1973, S. 154-155. Zum Zusammenhang von "geschlechtsspezifischen" Berufen und der Einschätzung von Kompetenz siehe: N. T. Feather: Positive and Negative Reactions to Male and Female Success and Failure in Relation to the Perceived Status and Sex-Typed Appropriateness of Occupations, in: Journal of Personality and Social Psychology 31/1975, S. 536-548 sowie Teresa Peck: When Women Evaluate Women, Nothing Succeeds Like Success: The Differential Effects of Status Upon Evaluations of Male and Female Professional Ability, in: Sex Roles 4/1978, S. 205-213. Zur Rolle der Personalpronomen siehe auch: Marty M. Salter / Deborah Weider-Hatfield / Donald L. Rubin: Generic Pronoun Use and Perceived Speaker Credibility, in: Communication Quarterly 31/1983, S. 180-184. Denkbar, wenngleich von den Forschern offenbar nicht in Betracht gezogen, ist, daß in der Studie von Miller/McReynolds der gewählte weibliche Vorname eine zusätzliche Einflußgröße war. Ausschlaggebend für die geringere Glaubwürdigkeit könnte hier neben dem Geschlecht sowohl die verniedlichende Form als auch die deutsche Herkunft sein.

Vgl. Erika Engstrom: Effects of Sex and Appearance of Ratings of Source Credibility. Unpublished Doctoral Dissertation, University of Florida 1991.

129 Vgl. Craig Johnson / Larry Vinson: "Damned if You Do, Damned if You Don't?" Status, Powerful Speech, and Evaluations of Female Witnesses, in: Women's Studies in Communication 10/1987, S. 37-44. 
und expressive Betonung) erbrachten bessere Glaubwürdigkeitszuschreibungen, wenn sie von Frauen, aber auch wenn sie von Männern verwendet wurden, während spezifisch "männliche" Sprechmuster (schlampige Aussprache, Unterbrechungen, niedrige Tonhöhe, keine ausgeprägte Betonung), unabhängig vom Geschlecht des Verwenders, zu niedrigeren Glaubwürdigkeitseinschätzungen führten. ${ }^{130}$

Der Nachweis, daß die Überzeugungskraft von Männern und Frauen davon abhängt, ob sie über "männerspezifische" oder "frauenspezifische" Themen urteilen, wird von Feldman-Summers et al. erbracht. Bei den vorgelegten Statements wurde dabei keine Fachkompetenz, sondern eine Kompetenz aufgrund persönlicher Erfahrung unterstellt. Bei "männerspezifischen" Themen ließen sich die Versuchspersonen eher von Männern beeinflussen, bei "frauenspezifischen" von Frauen. ${ }^{131}$

Schließlich zeigen Untersuchungen von Werbespots, daß die Geschlechter verschiedene Glaubwürdigkeitsdimensionen repräsentieren: Männliche Quellen werden als kompetenter, weibliche als vertrauenswürdiger eingestuft. ${ }^{132}$ Ein Grund für diese Einstufung liegt in den unterschiedlichen Rollen, die den Geschlechtern von den Werbetreibenden zugewiesen werden: Männer treten

130 Vgl. Cynthia L. Berryman-Fink / James R. Wilcox: A Multivariate Investigation of Perceptual Attributions Concerning Gender Appropriateness in Language, in: Sex Roles 9/1983, S. 663-681.

131 Vgl. Shirley Feldman-Summers et al.: Influence Attempts When Competing Views are Gender-Related: Sex as Credibility, in: Psychology of Women Quarterly 5/1980, S. 311-320. Geprüft wurden die Statements:

male-related: "Conscription and involontary military service tend to make a man bitter and angry." "Sexual intercourse will weaken a male athlete, and thus he should abstrain from sexual activity prior to participation in a sports event."

female-related: "Women tend to be monogamous by nature." "Most women fear for their safety when they walk alone at night, even in their own neighborhoods." (Vgl. S. 314)

132 Vgl. Sheila Brownlow / Leslie A. Zebrowitz: Facial Appearance, Gender, and Credibility in Television Commercials, in: Journal of Nonverbal Behavior 14/1990, S. 51-60; L. Z. McArthur / B. G. Resko: The Portrayal of Men and Women in American Television Commercials, in: Journal of Social Psychology 97/1975, S. 209-219. 
bevorzugt als Sachverständige auf, Frauen als Produktbenutzer. ${ }^{133}$

\subsubsection{Einflußfaktor Rezipient: Persönlichkeitsspezifische Merkmale}

Bislang wurde kaum untersucht, ob und inwieweit die jeweiligen Dimensionen bei verschiedenen Rezipienten einen unterschiedlichen Stellenwert haben. Hingegen wurde die Frage gestellt, ob und warum bestimmte Personen leichter durch glaubwürdige Quellen zu beeinflussen sind als andere.

Zur Erklärung für die unterschiedliche Bedeutung, die verschiedene Personen den Quellen zukommen lassen, wurde der 'Locus of Control' herangezogen. Unter Bezug auf Rotter wird dabei unterschieden zwischen Personen mit einem externen 'Locus of Control', die sich für fremdbestimmt und abhängig von Zufall, Schicksal oder Mächtigen halten und Personen mit einem internen 'Locus of Control', die sich als selbstbestimmt und eigenverantwortlich sehen. ${ }^{134}$ Gemäß dieser Unterscheidung wird die Vermutung formuliert, daß Personen, die sich einer externen Kontrolle unterworfen sehen, leichter durch glaubwürdige Quellen zu beeinflussen sind, während sich intern Kontrollierte sowohl von glaubwürdigen als auch von unglaubwürdigen Quellen in geringerem Ausmaß beeinflussen lassen und sich stärker der Mitteilung zuwenden. ${ }^{135}$

133 Vgl. A. Manstead / C. McCulloch: Sex-Role Stereotyping in British Television Advertisements, in: British Journal of Social Psychology 20/1981, S. 171-180; Adrian Furnham / Sandra Schofield: Sex-Role Stereotyping in British Radio Advertisements, in: British Journal of Social Psychology 25/1986, S. 165-171; Adrian Furnham / Virginia Voli: Gender Stereotypes in Italian Television Advertisements, in: Journal of Broadcasting \& Electronic Media 33/1989, S. 175-185; Adrian Furnham / Nadine Bitar: The Stereotyped Portrayal of Men and Women in British Television Advertisements, in: Sex Roles 29/1993, S. 297-310.

134 Vgl. Julian B. Rotter: General Expectancies for Internal versus External Control of Reinforcement, in: Psychological Monographs 80/1966, S. 1-28.

135 Vgl. Brian Sternthal/ Lynn W. Phillips / Ruby Dholakia: The Persuasive Effect of Source Credibility: A Situational Analysis, in: Public Opinion Quarterly 42/1978, S. 285-314, S. 295; Jean Charles Chebat / Pierre Filiatraut / Jean Perrien: Limits of Credibility: The Case of Political Persuasion, in: Journal of Social Psychology 130/1990, S. 157-167, S. 160. 
Die Thesen wurden in verschiedenen Untersuchungen bestätigt. ${ }^{136}$

Ein weiterer Ansatz arbeitet heraus, daß es Personen gibt, die eher quellenorientiert sind und solche, die eher mitteilungsorientiert sind. ${ }^{137}$ Erstere lassen sich bevorzugt von Variablen wie 'Sympathie' und 'Attraktivität' in ihren Einstellungsänderungen beeinflussen, letztere eher von der Art und Qualität der

136 Vgl. Elaine Ritchie / E. J. Phares: Attitude Change as a Function of Internal-External Control and Communicator Status, in: Journal of Personality 37/1969, S. 429-443; Elliott McGinnies / Charles D. Ward: Persuasibility as a Function of Source Credibility and Locus of Control: Five Cross Cultural Experiments, in: Journal of Personality 42/1974, S. 360-371.

137 Die Art der Orientierung hängt zu einem gewissen Teil ab von den Kommunikationsmustern, mit denen ein Rezipient innerhalb der Familie konfrontiert wurde. (Vgl. Vernon A Stone / Steven H. Chaffee: Familiy Communication Patterns and Source-Message Orientation, in: Journalism Quarterly 47/1970, S. 239-246.) Andere Autoren sehen einen Zusammenhang mit Adornos "Autoritärer Persönlichkeit". (Vgl. Theodor W. Adorno et al.: The Authoritarian Personality, New York 1950.) Demnach sind autoritäre Persönlichkeiten eher quellenorientiert und deshalb eher von Glaubwürdigkeitsmerkmalen beeinflußt, weniger autoritäre Personen eher mitteilungsorientiert. Der Zusammenhang wurde teils bestätigt (vgl. Homer H. Johnson / I. D. Steiner: Some Effects of Discrepancy Level on Relationships between Authoritarianism and Conformity, in: Journal of Social Psychology 73/1967, S. 199-204; J. Harvey / D. Hays: Effect on Dogmatism and Authority of the Source of Communication upon Persuasion, in: Psychological Reports 30/1972, S. 119-122), teils widerlegt (vgl. Homer H. Johnson / James M. Torcivia / Mary Ann Poprick: Effects of Source Credibility on the Relationship between Authoritarianism and Attitude Change, in: Journal of Personality and Social Psychology 9/1968, S. 179-183; Homer H. Johnson / Richard R. Izzett: Relationship between Authoritarianism and Attitude Change as a Function of Source Credibility and Type of Communication, in: Journal of Personality and Social Psychology 13/1969, S. 317-321).

Zur Erklärung für die unterschiedliche Orientierung wird ferner Rokeachs Persönlichkeitsvariable der "Aufgeschlossenheit" herangezogen. (Vgl. Milton Rokeach: The Open and Closed Mind, New York 1960.) Rokeachs Überlegungen stellen ein alternatives Konzept zur 'Autoritären Persönlichkeit dar, da der Schwerpunkt der Betrachtung nicht auf motivationstheoretischen Konstrukten, sondern auf individuellen kognitiven Strukturen liegt. (Vgl. Bernd Schäfer / Bernd Six: Sozialpsychologie des Vorurteils, Stuttgart u.a. 1978, S. 31.) Demnach sind aufgeschlossene Individuen besser in der Lage, zwischen Inhalt und Quelle einer Mitteilung zu unterscheiden als engstirnige Individuen. (Vgl. Frederic A. Powell: Open- and Closed-Mindedness and the Ability to Differentiate Source and Message, in: Journal of Abnormal and Social Psychology 65/1962, S. 61-64, S. 63.) Siehe dazu auch: James Rotton / Brian F. Blake / Richard Heslin: Dogmatism, Trust, and Message Acceptance, in: Journal of Psychology 96/1977, S. 81-88; Eleanor L. Norris: Attitude Change as a Function of Open or Closed-Mindedness, in: Journalism Quarterly 42/1965, S. 571-575. 
Argumentation. ${ }^{138} \mathrm{Zu}$ welcher dieser Gruppen jemand gehört, ermitteln Stone/Hoyt, indem sie den Versuchspersonen eine allgemeine Themenskala vorgeben (z.B. Politik, Bürgerrechte, medizinische Forschung, Gesundheitsfragen, Religion, Raumfahrt). Dann lassen sie die Versuchspersonen auf einer siebenstufigen Skala für jedes Thema bestimmen, ob es für sie jeweils wichtiger ist, wer dazu etwas sagt oder was dazu gesagt wird. Diejenigen, die sich häufiger für das 'Wer' entscheiden, gelten als quellenorientiert, diejenigen, denen das 'Was' wichtiger ist, gelten als mitteilungsorientiert; ferner gibt es noch eine Gruppe, die sich an beiden Elementen gleichermaßen orientiert und nicht eindeutig zugeordnet werden kann. ${ }^{139}$

Zusätzlich mußten die Probanden auf einer Liste die Eigenschaften markieren, die sie bei anderen Menschen am meisten schätzen oder ablehnen. Im anschließenden Experiment wurde dann einerseits die Sympathie für die Quelle manipuliert (indem man angab, daß die Quelle ähnliche oder unähnliche Eigenschaftspräferenzen hat wie die Versuchsperson) und andererseits der Informationsgehalt der Mitteilung variiert. Die so manipulierte Wahrnehmung der Ähnlichkeit zwischen Quelle und Proband führte bei quellenorientierten Personen deutlich häufiger zu einer Meinungsänderung als bei

138 Vgl. M. Thomas Milbourn / Vernon A. Stone: Source-Message Orientation and Components of Source Credibility, in: Journalism Quarterly 49/1972, S. 663-668, S. 663. Siehe auch: Vernon A. Stone / James L. Hoyt: Source-Message Orientation and Expectation of Discussion with the Source, in: Journalism Quarterly 48/1971, S. 741-744 und Vernon A. Stone: Individual Differences and Inoculation Against Persuasion, in: Journalism Quarterly 46/1969, S. 267-273. In eine ähnliche Richtung zielen Schulman/Worrall: Sie unterscheiden Personen, die spontane Assoziationen von Quellen und Inhalt vornehmen von solchen, die sich nur an den Inhalt erinnern; allerdings gehen sie nicht der Frage nach, woran das liegen könnte. (Vgl.Gary I. Schulman / Chrysoula Worrall: Salience patterns, Source Credibility, and the Sleeper Effect, in: Public Opinion Quarterly 34/1970, S. 371-382.

Vgl. Stone / Hoyt (1974b), S. 92f. In eine ähnliche Richtungen zielen die Überlegungen von Elliot R. Siegel / Gerald R. Miller / C. Edward Wotring: Source Credibility and Credibility Proneness. A New Relationship, in: Speech Monographs 36/1969, S. 118-125. Die Verfasser konstatieren, daß es Rezipienten gibt, die zwischen verschiedenen Quellen kaum differenzieren und sie als ähnlich wahrnehmen, und andere, die für die Unterschiede zwischen mehr und weniger akzeptablen Quellen sensibler sind. Letztere zeigen eine stärkere Neigung, sich an Glaubwürdigkeitskriterien zu orientieren und sich durch sie beeinflussen zu lassen. 
mitteilungsorientierten. Die Annahme, daß ein größerer Informationsgehalt bei mitteilungsorientierten Personen in ausgeprägterem Maße zu Meinungsänderungen führt, ließ sich jedoch nicht eindeutig bestätigen. ${ }^{140}$

In einem weiteren Experiment wurde zusätzlich zur Sympathie (die hier mittels verschiedener Eigenschaftszuschreibungen manipuliert wurde) die Kompetenzrelevanz ("relevance of expertness") variiert, also die sachliche Entsprechung von Kompetenz der Quelle und Thema der Aussage. ${ }^{141}$ Es wird davon ausgegangen, daß Kompetenz eher mitteilungsorientierte Rezipienten beeinflußt, da Kompetenz eine Beziehung zwischen der Quelle und der Mitteilung definiert ('Weiß der Kommunikator, wovon er spricht?'). ${ }^{142}$ Während die Bedeutung des Faktors 'Sympathie' für die quellenorientierten Rezipienten erneut nachgewiesen werden konnte, ergab die Kompetenzrelevanz für keine der beiden Personengruppen signifikante Ergebnisse. ${ }^{143}$ Vor allem aus dem Antwortverhalten der Personen, die sich nicht eindeutig einer der beiden Gruppen zuordnen lassen, leiten Stone/Hoyt ab, daß Quellen- und Mitteilungsorientierung keine sich ausschließenden Gegensätze sind, sondern daß beide eine Rolle spielen. ${ }^{144}$ Zusammenfassend stellen die Forscher fest:

$140 \quad$ Vgl. Stone / Hoyt (1974b), S. $93 \mathrm{ff}$.

141 Vgl. Stone / Hoyt (1974b), S. 101f. und Vernon A. Stone / James L. Hoyt: Effect of Likability and Relevance of Expertness, in: Journalism Quarterly 51/1974, S. 314-317 [1974a]. Zum Thema 'Zulassen von Fernsehkameras bei Gerichtsverhandlungen' hatte hohe Kompetenzrelevanz ein Juraprofessor, der sich mit Medienrechtsfragen beschäftigte, und geringe Kompetenzrelevanz ein Englischprofessor, dessen Forschungsschwerpunkt das Elisabethanische Drama war.

142 Vgl. Milbourn / Stone (1972), S. 664. Entsprechend wird hier vermutet (und bestätigt) daß Vertrauenswürdigkeit eher quellenorientierte Rezipienten beeinflußt, da sie eine Beziehung zwischen Quelle und Rezipient definiert ('Sagt mir der Kommunikator die Wahrheit, so wie er sie sieht?').

143 Vgl. Stone / Hoyt (1974b), S. 107f. Die Forscher führen das darauf zurück, daß die Manipulation der Kompetenzrelevanz für die Versuchspersonen (High-School-Schüler) nicht prägnant genug war und sie den Englischprofessor ebenfalls als hoch kompetent einstuften. 
"It may be that nearly everyone is more or less oriented to the message, which can be seen as the communication itself. (...) Source orientation ... appears to be more crucial than message orientation as a variable in persuasibility. This should not imply that differences in message orientation, though less pronounced, are less important." 145

Eine frühere Untersuchung, die von einem ähnlichen Grundgedanken ausgeht und ebenfalls zwischen der Orientierung an der Quelle und am Inhalt unterscheidet, legt nahe, daß die Art der Orientierung nicht so eindeutig in der Persönlichkeit des Rezipienten verwurzelt ist, wie Stone und Hoyt das postulieren. Vielmehr wird die Frage der Orientierung in Zusammenhang gebracht mit der Kommunikationssituation und der Intensität der Einstellung gegenüber der Quelle.

Aus diesen Faktoren resultiert eine bestimmte Haltung des Rezipienten, die zu Identifikation mit oder Abgrenzung von der Quelle führt; der Rezipient muß also seinen eigenen Standpunkt definieren.

"Given then, a situation in which concern with self-definition is brought into play, the person is not particularly oriented to what the other says, to the details of his argument. Rather, he is oriented to what the other is, to the other's position in relation to the self; is he on my side or is he against me?" 146

Entsprechend lauten die Festlegungen:

"We can speak of the tendency to view one's relationship to the communicator in terms of its relevance to self-definition as source orientation. Source orientation is defined as an approach to the communication situation in which the relation of the source to the person's self-definition has the greatest motivational significance. It can be contrasted to content orientation, an approach to the communication situation in which the relation of communication content to the person's

$145 \quad$ Milbourn / Stone (1972), S. 668.

146 Herbert C. Kelman / Alice H. Eagly: Attitude toward the Communicator, Perception of Communication Context, and Attitude Change, in: Journal of Personality and Social Psychology 1/1965, S. 63-78, S.73. [Hervorhebungen im Original.] 
value system has the greatest motivational significance." ${ }^{147}$

Eine so entstandene starke Quellenorientierung führt dazu, daß der Inhalt der Aussage in enger Verbindung mit der Quelle gesehen wird und von den Quellencharakteristika überlagert werden kann: Wird die gleiche Mitteilung einmal einer "positiven" Quelle, die die Rezipienten als vertrauenswürdig und ähnlich wahrnehmen, zugeordnet und einmal einer "negativen", die die Gegenseite verkörpert, wird unter der Voraussetzung, daß die Akzeptanz oder Ablehnung stark ausgeprägt ist, der tatsächliche Inhalt in die "passende" Richtung uminterpretiert. Das bedeutet, Auffassungen, die der eigenen eigentlich nahestehen, werden, wenn sie von einer negativen Quelle kommen, als weiter entfernt von der eigenen Position wahrgenommen, und umgekehrt werden Auffassungen, die der eigenen widersprechen, eher akzeptiert, wenn die Quelle vorher als positiv identifiziert wurde.

Geht es dagegen in erster Linie darum, die Implikationen zu bestimmen, die das Gehörte für eigene Ziele, Wünsche oder Wertvorstellungen hat, dominiert die Orientierung am Inhalt. Der Rezipient trennt dann zwischen Quelle und Inhalt und achtet nicht darauf, ob Quelle und Inhalt kongruent sind. Eine ausschließliche Konzentration auf den Inhalt kann sich allerdings gelegentlich als dysfunktional erweisen: Wenn der Inhalt völlig losgelöst von der Quelle betrachtet wird, werden Hinweise auf den Kontext (etwa Intentionen der Quelle) vernachlässigt, so daß Fehlinterpretationen nicht ausgeschlossen sind. ${ }^{148}$ Nach dieser Vorstellung werden Quellencharakteristika wie 'Kompe-

147 Kelman / Eagly (1965), S. 74f. [Hervorhebungen im Original.]

148 Vgl. Kelman / Eagly (1965), S. 75f. Die Orientierung an Quellen oder Inhalten geht einher mit unterschiedlichen Prozessen, die Einstellungsänderungen verursachen und die KELMAN schon früher postulierte: Einstellungsänderungen kommen nach dieser Vorstellung zustande durch Identifikation (Einstellungen oder Verhalten werden übernommen, weil die Beziehung zum anderen in Zusammenhang mit einer befriedigenden Selbst-Definition steht) oder durch Internalisierung (Einstellungen oder Verhalten werden übernommen, weil sie in Übereinstimmung mit dem eigenen Wertesystem stehen). Identifikation ist kennzeichnend für quellenorientierte Personen, Internalisierung für inhaltsorientierte. (Vgl. Herbert C. Kelman: Process of Opinion Change, in: Public Opinion Quarterly 25/1961, S. 57-78 und ders.: 
tenz' und 'Vertrauenswürdigkeit', die primär Indizien für Glaubwürdigkeit sind, bevorzugt von inhaltsorientierten Personen herangezogen; quellenorientierte Personen stützen sich auf Merkmale wie Sympathie und Attraktivität. ${ }^{149}$

\subsubsection{Einflußfaktor Rezipient: Die Beziehung zum Thema}

Bei ihrer Unterscheidung in quellen- und mitteilungsorientierte Rezipienten berücksichtigen Stone/Hoyt nicht, daß die Mitteilung selbst für die Art der Orientierung ausschlaggebend sein könnte. Auch in anderen Untersuchungen zur Glaubwürdigkeit der Quelle wird häufig nicht in Betracht gezogen, daß die Einschätzung der Rezipienten auch davon abhängig ist, welche Bedeutung das Thema, über das gesprochen wird, für sie hat, obgleich bereits Hovland und Weiss darauf hinweisen, daß die Glaubwürdigkeitseinschätzungen der Rezipienten "... were also affected by their personal opinions on the topic before the communication was ever presented." 150

Im Zusammenhang mit der Erforschung von Einstellungsänderungen wird dieser Beziehung von Rezipient und Thema unter dem Schlagwort 'egoinvolvement' Rechnung getragen: Je wichtiger ein Thema für einen Rezipienten ist, desto schwieriger ist es, ihn zu einer Meinungsänderung zu bewegen. Seine eigene Meinung zum Thema fungiert als eine Art "Anker", von dem aus er einen Sprecher und dessen Mitteilung beurteilt. ${ }^{151}$

Compliance, Identification, and Internalization: Three Processes of Opinion Change, in: Journal of Conflict Resolution 2/1958, S. 51-60.)

149 Vgl. John Shaw: Some "Real-Life" Accounts of Influential Relationships, in: Human Relations 30/1977, S. 363-372, S. 364 und Daniel G. Linz / Steven Penrod: Increasing Attorney Persuasiveness in the Courtroom, in: Law and Psychology Review 8/1984, S. 1-47, S. 30. Diese Zuordnung deckt sich größtenteils mit der von Milbourn/Stone (1972: 664); lediglich bezüglich der Einstufung der Vertrauenswürdigkeit besteht Uneinigkeit.

$150 \quad$ Hovland / Weiss (1951), S. 641.

151 Vgl. Carolyn W. Sherif / Muzafer Sherif: Attitude, Ego-Involvement and Change, New York 1967, S. 132f. Gebräuchlich und als Synonyme zu betrachten sind auch die Bezeich- 
Gemessen wird die persönliche Beteiligung durch die Frage nach dem Interesse am Thema sowie nach Wissen bzw. Vorinformation ${ }^{152}$ oder daran, wie die Probanden bestimmte vorgegebene Statements zum Thema einordnen. $^{153}$

Eine weitere Möglichkeit ist die Manipulation im Rahmen der experimentellen Versuchssituation: Involvement wird erzeugt, indem man den Versuchspersonen, durch Zusatzinformationen suggeriert, daß sie persönlich betroffen sind. Zum Beispiel teilen Petty und Cacioppo den am Experiment beteiligten Studenten mit, daß die Änderung der Vorschriften, von denen im Text die Rede ist, an ihrer eigenen Universität eingeführt werden sollen. ${ }^{154}$

Aus dem Befund, daß hohes 'issue involvement' zu erhöhter Akzeptanz der Mitteilung führt, die die eigenen Einstellungen stützt und zu verminderter Zustimmung zu den Positionen, die den eigenen Einstellungen entgegenstehen $^{155}$, leiten Petty/Cacioppo im Blick auf die Glaubwürdigkeit ab:

nungen issue involvement und personal involvement. Davon abzugrenzen ist das Phänomen des response involvement oder task involvement: Hier ist das Thema für den Rezipienten zwar nicht besonders wichtig, aber das Einnehmen einer bestimmten Haltung zum Thema führt zu sofortiger Belohnung. (Vgl. Richard E. Petty / John T. Cacioppo: Issue Involvement Can Increase or Decrease Persuasion by Enhancing Message-Relevant Cognitive Responses, in: Journal of Personality and Social Psychology 37/1979, S. 1915-1926. [Nachfolgend zitiert als 1979b.]

So etwa bei Elliott McGinnies: Studies in Persuasion: IV. Source Credibility and Involvement as Factors in Persuasion with Students in Taiwan, in: Journal of Social Psychology 74/1968, S. 171-180 sowie ders.: Initial Attitude, Source Credibility, and Involvement as Factors in Persuasion, in: Journal of Experimental Social Psychology 9/1973, S. 285-296.

153 Vgl. Kenneth K. Sereno: Ego-Involvement, High Source Credibility, and Response to a Belief-Discrepant Communication, in: Speech Monographs 35/1968, S. 476-481.

Vgl. Petty/Cacioppo (1979b), S. 1917. In einer anderen Versuchsanordnung wird behauptet, daß das beworbene Produkt demnächst in ortsansässigen Supermärkten erhältlich sein soll. (Vgl. Pamela M. Homer / Lynn R. Kahle: Source Expertise, Time of Source Identification, and Involvement in Persuasion: An Elaborative Processing Perspective, in: Journal of Advertising 19/1990, S. 30-39.) 
"... that nonmessage clues such as the expertise or attractiveness of a source should have maximal impact when persuasion is not tied to an extensive processing of the message content, as when a message is on a topic of low involvement. On the other hand, characteristics of the message content should have maximal impact under high involvement conditions." 156

Diese These erfährt Bestätigung durch die Befunde von McGarry und Hendrick: Starke persönliche Beteiligung führt dazu, daß weder Kompetenz noch Vertrauenswürdigkeit der Quelle einen Einfluß ausüben und die Versuchspersonen sich statt dessen in erster Linie auf den Inhalt der Mitteilung konzentrieren. ${ }^{157}$ Umgekehrt stützen die Ergebnisse von Lashbrook et al. die Annahme, daß Personen, die wenig Vorwissen sowie geringes Interesse am Thema haben, Informationen über die Glaubwürdigkeit der Quellen zur Bestimmung des Informationsgehalts heranziehen. ${ }^{158}$

Untersuchungen zur Glaubwürdigkeit von Quellen, die die persönliche Betroffenheit der Rezipienten berücksichtigen, gelangen weitgehend übereinstimmend zu dem Ergebnis, daß sich die meisten Einstellungsänderungen bei geringem 'Involvement' und hoher Glaubwürdigkeit der Quelle einstellen; bei hohem 'Involvement' stellen sich kaum Einstellungsänderungen ein, Unterschiede hinsichtlich der Glaubwürdigkeit von Quellen spielen dabei kaum eine Rolle. ${ }^{159}$ Lediglich Sereno berichtet, daß sowohl stark als auch gering

$156 \quad$ Petty / Cacioppo (1979b), S. 1924.

157 Vgl. James McGarry / Clyde Hendrick: Communicator Credibility and Persuasion, in: Memory \& Cognition 2/1974, S. 82-86.

158 Vgl. William B. Lashbrook / William B. Snavely / Daniel L. Sullivan: The Effects of Source Credibility and Message Information Quantity on the Attitude Change of Apathetics, in: Communication Monographs 44/1977, S. 252-262.

Vgl. McGinnies (1968 und 1973) sowie Homer H. Johnson / John A. Scileppi: Effects of Ego-Involvement Conditions on Attitude Change to High and Low Credibility Communicators, in: Journal of Personality and Social Psychology 13/1969, S. 31-36; Ramon J. Rhine / Laurence J. Severance: Ego-Involvement, Discrepancy, Source Credibility, and Attitude Change, in: Journal of Personality and Social Psychology 16/1970, S. 175-190; Gerald J. Gorn: The Effects of Personal Involvement, Communication Discrepancy, and Source Prestige on Reactions to Communications on Separatism, in: Canadian Journal of Behavioral Science 7/1975, S. 369386. Richard E. Petty / John T. Cacioppo / Rachel Goldman: Personal Involvement as a 
Involvierte ihre Einstellungen unter dem Einfluß einer Quelle mit hoher Glaubwürdigkeit ändern, wenngleich letztere dies in weit stärkerem Ausmaß tun.

"One possible reason for the small but statistically significant shifts on the part of highly involved subjects is that high credibility of the source interacted with level of involvement to lessen the anchoring strength of highly involved subjects' own position on the topic. The influence of ego-involvement upon response can seemingly be modified through interaction with the variable of high source credibility." 160

Dem Einfluß einer Quelle mit hoher Glaubwürdigkeit können sich Personen, die vom Thema betroffen sind, offenbar nicht entziehen, obwohl sie -im Gegensatz zu Uninteressierten - dazu neigen, die Quelle als weniger glaubwürdig anzusehen, wenn sie entgegengesetzte Standpunkte vertritt. ${ }^{161}$ Wird die Glaubwürdigkeit der Quelle nicht angezweifelt, führt eine hohe Glaubwürdigkeit dazu, daß eine intensivere Auseinandersetzung mit den Inhalten der Mitteilung erfolgt, da die Argumente nicht ohne weiteres negiert werden können. ${ }^{162}$

Als Erklärungsversuch für den unterschiedlichen Stellenwert der Quellen in Abhängigkeit vom persönlichen 'Involvement' der Rezipienten kann das 'Elaboration Likelihood Model' von Petty und Cacioppo herangezogen werden, das unterschiedliche Möglichkeiten der Informationsverarbeitung aufzeigt. Danach können Rezipienten auf zweierlei Arten mit persuasiver

Determinant of Argument-Based Persuasion, in: Journal of Personality and Social Psychology 41/1981, S. 847-855. ment, and Action, in: Journal of Personality and Social Psychology 27/1973, S. 311-328.

162 Vgl. Martin Heesacker / Richard E. Petty / John T. Cacioppo: Field Dependence and Attitude Change: Source Credibility Can Alter Persuasion by Affecting Message-Relevant Thinking, in: Journal of Personality 51/1983, S. 653-666, S. 663. 
Kommunikation umgehen: Entweder versuchen sie mit beträchtlichem Aufwand die Validität des Kommunikationsinhalts zu bestimmen oder sie verarbeiten eine Mitteilung sehr schnell, wobei sie sich auf nichtinhaltliche Kriterien wie die Glaubwürdigkeit der Quelle stützen. Im ersten Fall ziehen sie zentrale Hinweise ('central cues') zur Informationsverarbeitung heran, wobei sie dies nur dann tun, wenn die persönliche Motivation hoch ist und ausreichende Kenntnisse des in Frage stehenden Sachverhalts vorhanden sind. Im zweiten Fall - bei geringem 'Involvement' - begnügen sie sich mit 'peripheral cues' wie sie Quellencharakteristika darstellen. ${ }^{163}$ Diese Verarbeitungsstrategien zeichnen sich auch ab bei Personen, deren Einstellungen zu einem Gegenstand auf eigener oder indirekt vermittelter Erfahrung beruhen: Erstere wählen, wenn sie mit einer abweichenden Auffassung konfrontiert werden, eine zentrale Verarbeitungsroute, halten die betreffende Quelle für weniger glaubwürdig, produzieren mehr Gegenargumente und lassen sich weniger beeinflussen; letztere orientieren sich an den Glaubwürdigkeitskriterien und schließen sich eher der Auffassung eines glaubwürdigen Kommunikators an. ${ }^{164}$ Bislang ist noch ungeklärt, ob im Rahmen dieser Modellvorstellung bestimmte Eigenarten der Quelle - wie etwa die Kompetenz - unter bestimmten Bedingungen auch als zentrale Hinweise dienen können. ${ }^{165}$ Daneben ist denkbar, daß eine Quelle mit

163 Vgl. Richard E. Petty / John T. Cacioppo: Source Factors and the Elaboration Likelihood Model of Persuasion, in: Thomas Kinear (Hrsg.): Advances in Consumer Research, Vol. 11, Provo, UT. 1985, S. 668-672; dies.: The Elaboration Likelihood Model of Persuasion, in: Leonard Berkowitz (Hrsg.): Advances in Experimental Social Psychology, Vol. 19, New York/London 1986, S. 123-205 [1986b]; dies.: The Effects of Involvement on Responses to Argument Quantity and Quality: Central and Periphal Routes to Persuasion, in: Journal of Personality and Social Psychology 46/1984, S. 69-81; dies.: Involvement and Persuasion: Tradition versus Integration, in: Psychological Bulletin 107/1990, S. 367-374; Richard R. Petty / John T. Cacioppo / David Schumann: Central and Peripheral Routes to Advertising Effectiveness: The Moderating Role of Involvement, in: Journal of Consumer Research 10/1983, S. 135-146.

164 Vgl. Chenghuan Wu / David R. Shaffer: Susceptibility to Persuasive Appeals as a Function of Source Credibility and Prior Experience With the Attitude Object, in: Journal of Personality and Social Psychology 52/1987, S. 677-688. 
hoher Glaubwürdigkeit die Rezipienten eher dazu anregt, sich mit dem Textinhalt auseinanderzusetzen als eine weniger glaubwürdige Quelle. ${ }^{166}$

Eine ähnliche Vorstellung liegt dem Heuristisch-Systematischen Modell von Chaiken zugrunde. Sie unterscheidet zwischen systematischen und heuristischen Strategien der Informationsverarbeitung, die für den Umgang mit persuasiver Kommunikation heranzuziehen sind. Bei systematischer Informationsverarbeitung werden alle eingehenden Informationen auf ihre Relevanz für die Beurteilung eines Sachverhalts geprüft, während sich bei heuristischer Informationsverarbeitung die Rezipienten ausschließlich auf den Informationsausschnitt konzentrieren, der es ihnen ermöglicht, einfache Schlußfolgerungsregeln oder Schemata anzuwenden, um zu Urteilen zu gelangen. Solche Urteilsheuristiken werden vor allem dann angewendet, wenn Mitteilungen sehr komplex sind oder wenn ihre Inhalte von geringer persönlicher Bedeutung sind. Die Entscheidungsregeln basieren auf früheren Erfahrungen und Beobachtungen. Entsprechend orientieren sich Rezipienten bei heuristischer Informationsverarbeitung an Merkmalen des Kommunikators, aber auch an Merkmalen der Mitteilung (z.B. Plausibilität). Systematische Informationsverarbeitung dagegen erfordert die aktive und intensive Auseinandersetzung mit dem Inhalt der Mitteilung. Der erhebliche Aufwand, den systematische Informationsverarbeitung verlangt, wird nach dieser Vorstellung ebenfalls nur dann

tion, and Involvement in Persuasion: An Elaborative Processing Perspective, in: Journal of Advertising 19/1990, S. 30-39. Siehe dazu auch die Kritik von James B. Stiff: Cognitive Processing of Persuasive Message Cues: A Meta-Analytic Review of the Effects of Supporting Information on Attitudes, in: Communication Monographs 53/1986, S. 75-89. Hier wird dargelegt, daß im Fall von hohem 'Involvement' Rezipienten durchaus beide Wege der Informationsverarbeitung wählen können und sich entsprechend bis zu einem gewissen Grad auch auf Glaubwürdigkeitscharakteristika der Quelle stützen.

166 Vgl. Jeffery J. Mondak: Perceived Legitimacy of Supreme Court Decisions: Three Functions of Source Credibility, in: Political Behavior 12/1990, S. 363-384, S. 366 und Cal D. Stoltenberg / Carl S. Davis: Career and Study Skills Information: Who says What Can Alter Message Processing, in: Journal of Social and Clinical Psychology 6/1988, S. 38-52, S. 48. Siehe auch Karl E. Weick / David P. Gilfillan / Thomas A. Keith: The Effect of Composer Credibility on Orchestra Performance, in: Sociometry 36/1973, S. 435-462. 
unternommen, wenn das Thema für den Rezipienten wichtig ist oder aber, wenn Anhaltspunkte für Mißtrauen vorhanden sind. ${ }^{167}$ Es gibt Hinweise, daß Merkmale des Kommunikators (etwa seine Attraktivität) oder Merkmale des Inhalts (etwa eine emotionale Tönung der Aussage) eine heuristische Informationsverarbeitung auslösen, während das Fehlen prägnanter Merkmale eine tendenziell systematische Verarbeitung nach sich zieht. ${ }^{168}$

\subsubsection{Einflußfaktor Mitteilung: Spezielle Merkmale von Stil und Inhalt}

Im Rahmen der quellenorientierten Glaubwürdigkeitsforschung wird den Inhalten der Mitteilung in der Regel keine Aufmerksamkeit geschenkt. Dies ist umso erstaunlicher, als aus der Tatsache, daß meist auch Quellen mit geringerer Glaubwürdigkeit für Meinungsänderungen (wenn auch in bescheidenem Umfang) sorgen, die naheliegende Vermutung abgeleitet werden kann, daß Inhalte durchaus eine Rolle spielen. ${ }^{169}$

167 Vgl. Shelly Chaiken: Heuristic Versus Systematic Information Processing and the Use of Source Versus Message Cues in Persuasion, in: Journal of Personality and Social Psychology 39/1980, S. $752-766$ und dies.: The Heuristic Model of Persuasion, in: Mark P. Zanna / James M. Olson / C. Peter Herman (Hg.): Social Influence: The Ontario Symposium, Vol. 5, Hillsdale, NJ. 1987, S. 3-39; sowie Shelly Chaiken / Alice H. Eagly: Communication Modalty as a Determinant of Message Persuasiveness and Message Comprehensibility, in: Journal of Personality and Social Psychology 34/1976, S. 605-614 und Shelly Chaiken / Alice H. Eagly: Communication Modalty as a Determinant of Persuasion: The Role of Communicator Salience, in: Journal of Personality and Social Psychology 45/1983, S. 241-256. Zur Bedeutung von Heuristiken vgl. auch den Überblick von Hans-Bernd Brosius: Alltagsrationalität in der Nachrichtenrezeption. Ein Modell zur Wahrnehmung und Verarbeitung von Nachrichteninhalten, Opladen 1995, S. 106ff.

Vgl. Suzanne R. Pallak: Salience of Communicator's Physical Attractiveness and Persuasion: A Heuristic versus Systematic Processing Interpretation, in: Social Cognition 2/1983, S. 158-170 und Suzanne R. Pallak / Enid Murroni / Juliann Koch: Communicator Attractiveness and Expertise, Emotional versus Rational Appeals, and Persuasion: A Heuristic versus Systematic Processing Interpretation, in: Social Cognition 2/1983, S. 122-141. Source Credibility, Attitudes, and the Recall of Past Behaviours, in: European Journal of Social Psychology 14/1984, S. 203-210. Die Autoren verweisen darauf, daß der von ihnen als Quelle mit niedriger Glaubwürdigkeit gedachte Sprecher (ein Schüler) in Pretests nicht als solcher 
Dennoch werden lediglich vereinzelt Sonderaspekte des Inhalts mit der Glaubwürdigkeit des Kommunikators in Zusammenhang gebracht. Variiert werden vor allem formale und stilistische Textelemente. ${ }^{170}$ Eine spezielle Verbindung zwischen Quelle und Inhalt der Mitteilung wird durch die Wortwahl hergestellt. Mika verweist darauf, daß sowohl ausgewiesene Experten als auch Laien häufiger als verläßlich und kompetent eingestuft werden, wenn sie Fachtermini anstelle von umgangssprachlichen Erklärungen benutzen. Er zieht daraus den Schluß, daß Rezipienten von Experten geradezu erwarten, daß die Fachkompetenz in der sprachlichen Manifestation durch die Verwendung einer Fachsprache ihren Ausdruck findet. ${ }^{171}$ Fluchen und der Gebrauch obszöner Wörter dagegen mindern die zugesprochene Glaubwürdigkeit:

"[The] findings suggest that a communicator contemplating the use of profanity as a vehicle designed to increase the persuasiveness of his message may be sadly disappointed in the obtained results. In fact, not only can he expect to be no more persuasive by including obscenities in his presentation, but he can also expect some diminuition of his perceived 'safety'. Further, it will be recalled that when the credibility ratings for the combined profanity-type treatment means were compared with the comparable control group means, it was found that in all instances that the source using profanity (of any type) was perceived less credible than was

wahrgenommen wurde, "probably because he seemed very well-informed in the interview" (S 205).

170 Der Vorwurf, solche Beglaubigungs-Elemente würden von der Wissenschaft vernachlässigt, den Wagnererhebt, ist also in dieser Absolutheit nicht zutreffend. (Vgl. Hans Wagner: Das Fach-Stichwort: Das Grubenhund-Gesetz: Die Rationalität der sozialen Orientierung, in: Artur Schütz: Der Grubenhund. Experimente mit der Wahrheit, hrsg. von Walter Hömberg, München 1996, S.119-192, S.166f.) Unbestreitbar ist aber, daß Artur Schütz mit seinen GrubenhundKonstruktionen eindrucksvolle Feldversuche liefert, in denen er intuitiv alle relevanten Glaubwürdigkeits-Komponenten vorwegnimmt (sein Buch erschien bereits 1931) und aufzeigt, daß selbst unsinnigste Inhalte die Selektionsbarrieren von Zeitungsredakteuren passieren, wenn sie durch geeignete Glaubwürdigkeits-Elemente legitimiert sind.

171 Vgl. Stanislaw Mika: Some Determinants of Source Credibility, in: Polish Psychological Bulletin 12/1981, S. 79-86, S. 81f. Siehe dazu auch Earl R. Carlson: Word Familiarity as a Factor in Forming Impressions, in: Psychological Reports 7/1960, S. 18. Dort zeigt CARLSON, daß ein Sprecher, der unbekannte Wörter verwendet, mehr respektiert wird, als einer, der sich der Alltagssprache bedient. 
the source not using profanity, and in the case of the Competence dimension ratings, was seen as significantly less credible." ${ }^{172}$

Neben sprachlichen Hinweisen auf Kompetenz können Indizien für Ähnlichkeit zwischen Sprecher und Empfänger, wie sie etwa durch die Verwendung von Dialekt gegeben sind, Einfluß auf die Glaubwürdigkeit eines Sprechers nehmen. ${ }^{173}$ Auch andere Ähnlichkeiten im Sprachgebrauch, wie die Neigung zu "intensivem" (wertgeladenem) Vokabular, beeinflussen die Wahrnehmung der Glaubwürdigkeit. ${ }^{174}$ Ohne den Aspekt der Ähnlichkeit zu prüfen, wird darüberhinaus konstatiert, daß die Intensität der Sprache die Überzeugungskraft glaubwürdiger Quellen erhöht und die weniger glaubwürdiger Quellen mindert. ${ }^{175} \mathrm{Zu}$ einer Erhöhung der Glaubwürdigkeit bei Experten trägt die Verwendung von Satzkonstruktionen im Passiv bei, weil dadurch Neutralität

172 Robert N. Bostrom / John R. Baseheart / Charles M. Rossiter, Jr.: The Effects of Three Types of Profane Language in Persuasive Messages, in: Journal of Communication 23/1973, S. 461-475, S. 472. Der Befund fällt allerdings lediglich für männliche Sprecher so eindeutig aus. Frauen wirken überzeugender und werden auch etwas glaubwürdiger wahrgenommen, wenn sie anstößige Wörter benutzen. (Vgl. ebd. S. 473f.)

173 Vgl. Miller / Hoppe (1973), S. $211 \mathrm{f}$.

174 Vgl. R. Kelly Aune / Toshiyuki Kikuchi: Effects of Language Intensity Similarity on Perceptions of Credibility, Relational Attributions, and Persuasion, in: Journal of Language and Social Psychology 12/1993, S. 224-238.

175 Vgl. Mark A. Hamilton/ John E. Hunter / Michael Burgoon: An Empirical Test of an Axiomatic Model of the Relationship Between Language Intensity and Persuasion, in: Journal of Language and Social Psychology 9/1990, S. 235-255. Intensität im Sprachgebrauch führt zu einer Verbesserung der Zuschreibung von 'Kompetenz', 'Vertrauenswürdigkeit' und 'Dynamik'. Gleichzeitig wird die Mitteilung als logischer und klarer eingestuft. (Vgl. William J. McEwen / Bradley S. Greenberg: The Effects of Message Intensity on Receiver Evaluations of Source, Message and Topic, in: Journal of Communication 20/1970, S. 340-350.) Berichtet werden aber auch gegenteilige Effekte: die Minderung von Glaubwürdigkeit durch eine emotional getönte Sprache. (Vgl. O. Wiegmann: two Politicians in a Realistic Experiment: Attraction, Discrepancy, Intensity of Delivery, and Attitude Change, in: Journal of Applied Psychology 15/1985, S. 687-699.) Solche Differenzen dürften allerdings in einer je unterschiedlichen Operationalisierung von 'Intensität' begründet sein. Abgesehen von McEwen/Greenberg, die einige Beispiele liefern, legen die Forscher nicht dar, auf welche Weise sie 'Intensität' erzielen. 
und Distanz suggeriert und die Sprecher damit als vertrauenswürdiger wahrgenommen werden. ${ }^{176}$

Die Verwendung von Metaphern oder Vergleichen kann die Glaubwürdigkeit des Sprechers ebenfalls positiv beeinflussen; die Uneinheitlichkeit der Befunde in diesem Bereich ${ }^{177}$ läßt allerdings den Schluß zu, daß dies nur unter bestimmten Bedingungen geschieht. Vermehrte Glaubwürdigkeit wird dem Kommunikator etwa zugeschrieben, wenn die verwendeten Bilder besonders eindringlich sind oder wiederholt und variiert werden. ${ }^{178}$

Auf dem Feld der vergleichenden Werbung hat sich die Strategie bewährt, dem eigenen Produkt nicht in allen Bereichen bessere Eigenschaften zuzuschreiben. Die Glaubwürdigkeit eines Werbetreibenden wird erhöht, wenn dem Konkurrenzprodukt wenigstens in einer Vergleichskategorie Überlegenheit bescheinigt wird. $^{179}$.

Eine inhaltsanalytische Bestandsaufnahme von Redetexten erbrachte, daß Glaubwürdigkeit in Zusammenhang mit verschiedenen stilistischen Elementen

176 Vgl. Steven D. Hurwitz / Murray S. Miron / Blair T. Johnson: Source Credibility and the Language of Expert Testimony, in: Journal of Applied Social Psychology 22/1992, 1909-1939, S. 1928.

177 Ein Einfluß der Verwendung von Metaphern auf die Glaubwürdigkeit wurde erstmals nachgewiesen durch John Waite Bowers / Michael M. Osborn: Attitudinal Effects of Selected Types of Concluding Metaphors in Persuasive Speeches, in: Speech Monographs 33/1966, S. 147-155. Demgegenüber fanden McCroskey/Combs zwar Auswirkungen auf Einstellungsänderungen, nicht aber auf die wahrgenommene Glaubwürdigkeit. (Vgl. James C. McCroskey / Walter H. Combs: The Effects of the Use of Analogy on Attitude Change and Credibility, in: Journal of Communication 19/1969, S. 333-339.)

Vgl. N. L. Reinsch, Jr.: Figurative Language and Source Credibility: A Preliminary Investigation and Reconceptualization, in: Human Communication Research 1/1974, S. 75-80, S. 75. Siehe auch ders.: An Investigation of the Effects of the Metaphor and Simile in Persuasive Discourse, in: Speech Monographs 38/1971, S. 142-145.

179 Vgl. Robert B. Settle / Linda L. Golden: Attribution Theory and Advertiser Credibility, in: Journal of Marketing Research 9/1974, S. 181-185. Zur Problematik der Glaubwürdigkeit bei vergleichender Werbung siehe auch Thomas E. Barry: Comparative Advertising: What Have We Learned in Two Decades? In: Journal of Advertising Research 33/1993, S. 19-29, S. $25 f$. 
steht: Texte, die als besser verstehbar klassifiziert wurden (gemessen wurde die durchschnittliche Anzahl der Wörter pro Satz), führten zu einer Zuschreibung hoher Glaubwürdigkeit, ebenso wie eine Personalisierung, die mittels häufiger Verwendung der Personalpronomen 'ich', 'wir' und 'Sie' ihren Ausdruck findet. Gleiches galt für ein variationsreiches Vokabular und eine geringe Neigung zur Abstraktion. ${ }^{180}$ Nachgewiesen ist ferner, daß ein schlecht strukturierter Vortrag die Glaubwürdigkeit des Sprechers mindert. ${ }^{181}$

Eine weitere inhaltliche Besonderheit stellen Verweise dar. Ostermeier konnte zeigen, daß Hinweise auf eigene Erfahrungen des Sprechers in Bezug auf das Thema (sogenannte 'self-references') die Wahrnehmung der Kompetenz und Vertrauenswürdigkeit erhöhen und zu verstärkter Einstellungsänderung führen, während die Dimension 'Dynamik' davon unberührt bleibt. Die Berufung auf Gewährsleute mit Erfahrung oder Kompetenz ('prestigereferences') führt demgegenüber lediglich zu einer erhöhten Vertrauenswürdigkeit. $^{182}$

Die Nennung von Gewährsleuten ist eine Möglichkeit, Belege oder Beweismaterial zur Stützung der eigenen Position anzuführen. Gebräuchlich sind daneben Augenzeugenberichte, Beispiele und statistische Daten. ${ }^{183}$ Als "Be-

180 Vgl. Tamara Carbone: Stylistic Variables as Related to Source Credibility: A Content Analysis Approach, in: Speech Monographs 42/1975, 99-106. Die Einschätzung erfolgte ausschließlich aufgrund der Textmerkmale; als Quellen wurden anonyme Studenten angegeben. Siehe dazu auch: James J. Bradac / Catherine W. Konsky / Robert A. Davies: Two Studies of the Effects of Linguistic Diversity upon Judgments of Communicator Attributes and Message Effectiveness, in: Communication Monographs 43/1976, S. 70-79. Hier wird der positive Einfluß eines abwechslungsreichen Vokabulars auf die Wahrnehmung der Dimensionen 'Kompetenz' und 'Dynamik' bestätigt. Gleichzeitig wurde ermittelt, daß Variationen auf syntaktischer Ebene, also im Gebrauch von Satzbau und Grammatik, keinen Einfluß auf die Zuschreibung von Glaubwürdigkeit hat.

181 Vgl. Eldon E. Baker: The Immediate Effects of Perceived Speaker Disorganization on Speaker Credibility and Audience Attitude Change in Persuasive Speaking, in: Western Speech 29/1965, S. 148-161.

182 Vgl. Terry H. Ostermeier: Effects of Type and Frequency of Reference upon Perceived Source Credibility and Attitude Change, in: Speech Monographs 34/1967, S. 137-144, S. 141ff.

183 Vgl. John C. Reinard: The Empirical Study of the Persuasive Effects of Evidence. The 
weise" werden aufgefaßt "factual statements originating from a source other than the speaker, objects not created by the speaker, and opinions of persons other than the speaker that are offered in support of the speaker's claims". ${ }^{184}$ Solche "Beweise" können (insbesondere bei Quellen mit ursprünglich niedriger Glaubwürdigkeit) zu einer Stärkung der Glaubwürdigkeit führen, während sich bei Quellen mit hoher Glaubwürdigkeit ein "Ceiling-Effekt" einstellt, das heißt, die zusätzliche Verwendung von Beweisen führt (zumindest kurzfristig) nicht $\mathrm{zu}$ einer stärkeren Einstellungsänderung. ${ }^{185}$ Auch eine Quelle mit hoher Glaubwürdigkeit kann aber nicht ohne weiteres auf Beweismaterial verzichten: Zumindest wenn Rezipienten zwei Seiten einer Kontroverse hören, sinkt die Glaubwürdigkeit einer ursprünglich hoch eingeschätzten Quelle, wenn sie - im Gegensatz zu ihrem Kontrahenten - keine Beweise präsentiert. ${ }^{186}$ Welcher Art das Beweismaterial ist, scheint - zumindest im Hinblick auf die Glaubwürdigkeit der Quelle - von untergeordneter Bedeutung: So ist es nicht von Belang, ob einzelne Fallbeispiele oder summarische Informationen in Form von Statistiken verwendet werden. $^{187}$ Hingegen wird in weitgehender Übereinstimmung konstatiert, daß Fallbeispiele überzeugender als Statistiken sind. ${ }^{188}$

Status After Fifty Years of Research, in: Human Communication Research 15/1988, S. 3-59, S. 13.

184 James C. McCroskey: A Summary of Experimental Research on the Effects of Evidence in Persuasive Communication, in: Quarterly Journal of Speech 55/1969, S. 169-176, S. 171.

185 Vgl. McCroskey (1969). Langfristig steigt allerdings die Überzeugungskraft von Quellen mit hoher und geringer Glaubwürdigkeit (vgl. Reinard (1988), S. 13.).

186 Vgl. James C. McCroskey: The Effects of Evidence as an Inhibitor of Counterpersuasion, in: Speech Monographs 37/1970, S. 188-194, S. 194.

187 Vgl. E. James Baesler/ Judee K. Burgoon: The Temporal Effects of Story and Statistical Evidence on Belief Change, in: Communication Research 21/1994, S. 582-602, S. 591

188 Vgl. zusammenfassend und mit ausführlichen Literaturhinweisen Reinard (1988), S. 13ff. und Brosius (1995), S. 238ff. Abweichende Befunde präsentieren lediglich Baesler / Burgoon (1994). 
Da Beweismaterial also häufig - vornehmlich wenn der Sprecher selbst kein Experte ist - in Kombination mit zitierten Quellen eingeführt wird, prüfte Cathcart, welchen Einfluß verschiedene Variationen der Präsentation von Beweisen und Quellen auf die Einstellungsänderung von Zuhörern haben. Zum Thema "Abschaffung der Todesstrafe" wurden vier Reden vorgelegt. Rede (A) bestand aus allgemeinen Aussagen zum Thema; Rede (B) untermauerte die Aussagen mit Beweismaterial, für das keine Quelle angegeben wurde; Rede (C) enthielt dasselbe Beweismaterial, mit Angaben von Personen oder Dokumenten sowie Ort und Datum der Bekanntgabe und in Rede (D) wurden zusätzlich Qualifikationsmerkmale der Quellen geliefert. Die Reden (B) und (D) führten zu den meisten Meinungsänderungen, während bloßes "name dropping" (Rede C) weniger erfolgreich war. ${ }^{189}$ Das angeführte Beweismaterial überzeugte also zum einen stark, wenn keine Namensnennung erfolgte - in diesem Fall wurde es wahrscheinlich dem Sprecher selbst zugeschrieben. Zum anderen ließen sich die Rezipienten stärker beeinflussen, sobald über die bloße Namensnennung der zitierten Quellen hinaus Hinweise auf deren Kompetenz gegeben wurde. ${ }^{190}$ Dies deutet auf eine Wechselwirkung von Kommunikationsinhalt und Quellen hin, die auch noch durch einen weiteren Befund nahegelegt wird: Für alle vier Redebedingungen wurde der Sprecher (der nicht vorgestellt wurde und nur via Tonband präsent war) als kompetent und qualifiziert eingestuft. Für die Bildung

189 Vgl. Robert S. Cathcart: An Experimental Study of the Relative Effectiveness of Four Methods of Presenting Evidence, in: Speech Monographs 22/1955, S. 227-233. Die Ergebnisse werden auch bestätigt bei: Robert N. Bostrom / Raymond K. Tucker: Evidence, Personality, and Attitude Change, in: Speech Monographs 36/1969, S. 22-27. Eine weitere Studie erbringt ebenfalls nur geringfügige, nicht signifikante Meinungsänderungen bei Nennung von Gewährsleuten: Vgl. Howard Gilkinson / Stanley F. Paulson / Donald E. Sikkink: Effects of Order and Authority in an Argumentative Speech, in: Quarterly Journal of Speech 40/1954, S. 183-192.

190 Ähnliche Ergebnisse berichtet auch Whitehead: Das Ausmaß der Einstellungsänderungen bleibt annähernd gleich, unabhängig davon, ob zitierte Quellen für Beweismaterial genannt werden oder nicht. Die Nennung der zitierten Quellen führt hier aber dazu, daß die sprechende Quelle als vertrauenswürdiger und objektiver eingestuft wird. (Vgl. Jack L. Whitehead, Jr.: Effects of Authority-Based Assertion on Attitude and Credibility, in: Speech Monographs 38/1971, S. 311-315.) 
dieser Urteile stand - neben den stimmlichen Qualitäten - nur der Inhalt der Mitteilung zur Verfügung. Hinweise, daß die Verwendung von Beweismaterial die Glaubwürdigkeit eines Sprechers stärken kann, wird auch von anderer Seite beigebracht. $^{191}$

Geprüft wurde ferner (vor allem im Bereich der Werbung) die Verwendung von Humor als Stilmittel. Eine Sichtung der Studien erbringt gemischte Befunde. Teils wird eine Stärkung, teils eine Schwächung der Glaubwürdigkeit der Quelle ermittelt, vermutlich in Abhängigkeit von der Art der Quelle und der Art des Humors. ${ }^{192}$ So ergibt sich ein leicht positiver Zusammenhang von Humor und Glaubwürdigkeit für männliche, nicht aber für weibliche Lehrer. ${ }^{193}$ Speck zeigt, daß die Verwendung von Humor generell die wahrgenommene Kompetenz senkt, daß aber bestimmte Arten von Humor die Vertrauenswürdigkeit der Quelle stärken. ${ }^{194}$ Auch eine Stärkung der Dimension 'Dynamik' ist nachgewiesen. ${ }^{195}$ Während die zentralen Glaubwürdigkeitsdimensionen aber

Vgl. Reinard (1988), S. 42; P. H. Bradley: The Folk-Linguistics of Women's Speech: An Empirical Examination, in: Communication Monographs 48/1981, S. 73-90; J. A. Kline: Interaction of Evidence and Readers' Intelligence on the Effects of Short Messages, in: Quarterly Journal of Speech 55/1969, S. 407-413; Irving D. Warren: The Effect of Credibility in Sources of Testimony on Audience Attitudes toward Speaker and Message, in: Speech Monographs 36/1969, S. 456-458. Das Zitieren glaubwürdiger Quellen führt bei Warren dazu, daß der Sprecher als deutlich fairer beurteilt wird, als wenn er wenig glaubwürdige Quellen zitiert.

192 Vgl. Marc G. Weinberger / Charles S. Gulas: The Impact of Humor in Advertising: A Review, in: Journal of Advertising 21/1992, S. 35-59, S. 42.

193 Vgl. Jennings Bryant et al.: Relationship Between College Teachers' Use of Humor in the Classroom and Students' Evaluation of Their Teachers, in: Journal of Educational Psychology $72 / 1980$, S. 511-519.

194 Vgl. Paul Surgi Speck: On Humor and Humor in Advertising. Unpublished Doctoral Dissertation, Texas Tech University 1987, zitiert nach: Weinberger / Gulas (1992), S. 49. Eine Stärkung der Dimension Vertrauenswürdigkeit berichtet auch Charles R. Gruner: Effect of Humor on Speaker Ethos and Audience Information, in: Journal of Communication 17/1967, S. 228-233.

195 Vgl. Allan James Kennedy: An Experimental Study of the Effect of Humorous Message Content Upon Ethos and Persuasiveness, Unpublished Doctoral Dissertation, University of Michigan 1972, zitiert nach: Weinberger / Gulas (1992), S. 47. 
häufig durch den Einsatz von Humor keine Veränderung erfahren, zeigt ein Literaturüberblick, daß die Sympathie, die der Quelle entgegengebracht wird, steigt. $^{196}$

\subsubsection{Zeitpunkt der Information}

Abgesehen von unterschiedlichen Orientierungsmustern auf Rezipientenseite gibt es Belege dafür, daß der Zeitpunkt der Information über die Quelle von Bedeutung ist. Mills und Harvey konnten zeigen, daß weniger Meinungsänderungen erfolgen, wenn die Kompetenzmerkmale erst mitgeteilt wurden, nachdem die Mitteilung rezipiert wurde. ${ }^{197}$

Husek führte dazu das folgende Experiment durch: Präsentiert wurde ein zwanzigminütiger Vortrag über Geisteskrankheiten, der so konzipiert war, daß er positive Einstellungen hervorrufen sowie Mißverständnisse und populäre Vorurteile abbauen sollte. Die Person, die den Vortrag hielt, wurde einmal nicht näher vorgestellt; einmal wurde sie zu Beginn und einmal am Ende des Vortrags als ehemals geisteskranke Patientin identifiziert. Der Forscher ging davon aus, daß diese Kennzeichnung geeignet war, die Quelle negativ zu beurteilen. Ferner nahm er an, daß eine frühe Vorstellung dieser negativen Quelle dazu führt, daß der Inhalt des Vortrags, der ja einseitig positiv argu-

196 Vgl. Weinberger / Gulas (1992), S. 44. Siehe dazu etwa Clark Leavitt: A Multidimensional Set of Rating Scales For Television Commercials, in: Journal of Applied Psychology 34/1970, S. 427-429; Betsy D. Gelb / Charles M. Pickett: Attitude-Toward-The-Ad: Links to Humor and to Advertising Effectiveness, in: Journal of Advertising 12/1983, S. 34-42; Betsy D. Gelb / George M. Zinkhan: Humor and Advertising Effectiveness after Repeated Exposures to a Radio Commercial, in: Journal of Advertising 15/1986, S. 15-20, 34.

Vgl. Judson Mills / John Harvey: Opinion Change as a Function of When Information about the Communicator is Received and Whether He Is Attractive or Expert, in: Journal of Personality and Social Psychology 21/1972, S. 52-55. Der Befund wird bestätigt bei Horai / Naccari / Fatoullah (1974) sowie D. J. O'Keefe: The Persuasive Effects of Delaying Identification on High- and Low-Credibility Communicators: A Meta-Analytic Review, in: Central States Speech Journal 38/1987, S. 63-72. 
mentierte, weniger starke Auswirkungen auf die Einstellung gegenüber Geisteskranken hat. Diese Annahme wurde bestätigt: Die Gruppen, denen die Identität der Quelle erst gegen Ende des Vortrags offenbart wurde, gaben deutlich positivere Einschätzungen ab. Husek schloß daraus, daß die Versuchspersonen in diesen Fällen schon durch die Argumentation überzeugt waren und ihre Einstellungen dann nicht mehr korrigierten, sondern die positiven Einstellungen auf die Quelle übertrugen. Eine frühe Identifikation der 'negativen' Quelle dagegen führte seiner Auffassung nach dazu, daß die negativen Einstellungen der Quelle gegenüber die Wahrnehmung der Mitteilung beeinflußten. ${ }^{198}$

Obwohl sie zunächst Kritik am methodischen Vorgehen von Husek üben, können Greenberg/Miller dessen Ergebnis in mehreren Experimenten bestätigen: ${ }^{199}$

"Individuals who heard a message following its attribution to a low-credible source seem to have been on their guard, to have been immunized against subsequent persuasion. In instances involving low-credible communicators, immediate identification appears to have served as a forewarning which alerted audience members to the fact that the message information might be unreliable. On the other hand, delayed identification of the source afforded an opportunity for audience members to evaluate and to respond to the message without the knowledge that its persuasive appeals came from a source whose competence and trustworthiness were questionable. It seems probable that persuasion occured before these individuals were apprised of the source's low credibility." 200

Die bisher genannten Studien verwenden ausschließlich Quellen mit niedriger

198 Vgl. T. R. Husek: Persuasive Impacts of Early, Late, or No Mention of a Negative Source, in: Journal of Personality and Social Psychology 2/1965, S. 125-128.

199 Vgl. Bradley S. Greenberg / Gerald R. Miller: The Effects of Low-Credible Sources on Message Acceptance, in: Speech Monographs 33/1966, S. 127-136, S. 128. Die Kritik bezieht sich darauf, daß keine unabhängige Einschätzung der Glaubwürdigkeit der Quelle erfolgte, sondern daß die geringe Glaubwürdigkeit vom Forscher unterstellt wurde.

200 Greenberg / Miller (1966), S. 135. Voraussetzung dabei ist allerdings, daß die Mitteilung selbst gewisse Anforderungen hinsichtlich Inhalt und Verständlichkeit erfüllt. 
Glaubwürdigkeit. Darüberhinaus konnte gezeigt werden, daß Quellen mit hoher Glaubwürdigkeit mehr Meinungsänderungen herbeiführen, wenn sie vor der Mitteilung präsentiert werden. ${ }^{201}$ Damit kann als gesichert gelten, daß der Zeitpunkt der Information über die Quelle die Wahrnehmung ihrer Glaubwürdigkeit beeinflußt. of Credible and Noncredible Sources, in: Journal of Psychology 86/1974, S. 17-23. 


\subsection{Der "sleeper effect"}

Der Faktor 'Zeit' wird auch noch in einem anderen Zusammenhang relevant: Das als "sleeper effect" bekannte Phänomen betrifft Unterschiede zwischen kurz- und langfristigen Kommunikationswirkungen.

Die Bezeichnung wurde von Hovland, Lumsdaine und Sheffield geprägt und geht auf folgende Beobachtung zurück: Armeeangehörigen wurde ein Propagandafilm ('The Battle of Britain') gezeigt, als dessen Quelle die Armee genannt wurde. Einige der Experimentalgruppen wurden eine Woche nach der Vorführung zum Film befragt, andere erst neun Wochen danach. Bei acht von 15 abgefragten Meinungspositionen war die Zustimmung zu der im Film vermittelten Sicht bei denjenigen Versuchspersonen größer, die später befragt wurden. Dieser langfristige Zuwachs wurde als "sleeper effect" bezeichnet. ${ }^{202}$

Ursprünglich ist damit also eine Meinungsänderung in die von der Quelle intendierte Richtung gemeint, die sich im Zeitverlauf einstellt. Der Kommunikationsinhalt wirkt sozusagen weiter, während die Rezipienten ihn "überschlafen". ${ }^{203}$ Im folgenden wurden der Effekt dann im Zusammenhang mit der Glaubwürdigkeit der Quelle betrachtet. In dem beschriebenen Experiment von Hovland und Weiss ${ }^{204}$ ging die Meinungsänderung, die auf die glaubwürdigen Kommunikatoren zurückzuführen war, nach vier Wochen zurück, während bei den Versuchspersonen, die mit wenig glaubwürdigen Quellen konfrontiert wurden, eine Meinungsänderung in die dort postulierte Richtung zu beobachten war. ${ }^{205}$ "Thus there was a forgetting effect when the presentation was by a trustworthiness communicator and a sleeper effect when the

\footnotetext{
202 Vgl. Carl I. Hovland / Arthur A. Lumsdaine / Fred D. Sheffield: Experiments in Mass Communication, New York 1949, S. 182-200.

203 Vgl. Paulette M. Gillig / Anthony G. Greenwald: Is it Time to Lay the Sleeper Effect to Rest? In: Journal of Personality and Social Psychology 29/1974, S. 132-139, S. 132.

204 Siehe Abschnitt C. 2., S. $32 \mathrm{ff}$.

205 Vgl. Hovland / Weiss (1951), S. 647.
} 
communication was presented by a negative communicator."206

Zunächst versuchte man, diese Befunde damit zu erklären, daß im Zeitverlauf die Quelle schneller vergessen wurde als der Inhalt ihrer Aussage, die Befragten sich also schlicht nicht mehr daran erinnerten, daß die Quelle unglaubwürdig war. Dieser Interpretationsversuch wurde jedoch widerlegt, da sich zeigte, daß die Versuchspersonen bei Nachfragen durchaus in der Lage waren, die Quellen zu nennen. Ein alternativer Erklärungsversuch formulierte die Annahme, daß Quelle und Aussage im Zeitverlauf "dissoziiert" werden, daß also die Personen, wenn sie nach ihrer Meinung gefragt werden, Quelle und Aussage nicht mehr miteinander in Verbindung bringen, obwohl das Wissen um die Quelle noch vorhanden ist. ${ }^{207}$ Wenn man den Versuchspersonen die Quelle erneut in Erinnerung ruft, stellt sich der ursprüngliche Abstand zwischen den Meinungspositionen wieder ein, das heißt, es kommt dann weder zu einem "sleeper effect" noch zu einem "forgetting effect". ${ }^{208}$

In einer Reihe weiterer Studien wird ebenfalls beansprucht, den Nachweis eines "sleeper effects" erbracht zu haben. ${ }^{209}$ Deren Ergebnisse werden jedoch von einigen Kritikern bezweifelt. ${ }^{210}$ Capon und Hulbert bemängeln vor allem

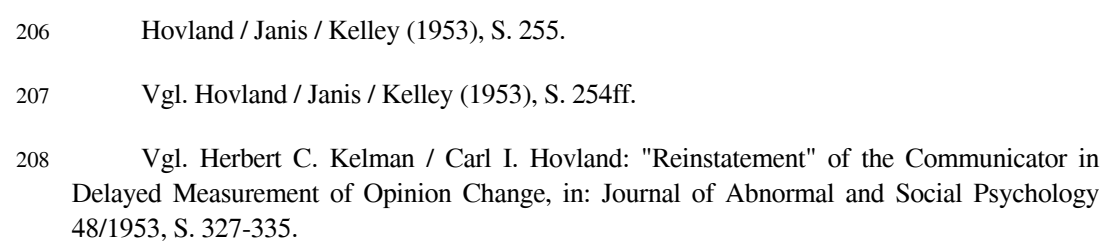
Delayed Measurement of Opinion Change, in: Journal of Abnormal and Social Psychology 48/1953, S. 327-335.

209 Vgl. Walter Weiss: A Sleeper Effect in Opinion Change, in: Journal of Abnormal and Social Psychology 48/1953, S. 173-180; Ezra Stotland / Daniel Katz / Martin Patchen: The Reduction of Prejudice through the Arousal of Self-Insight, in: Journal of Personality 27/1959, S. 507-531; Ezra Stotland / Martin Patchen: Identification and Changes in Prejudice and in Authoritarianism, in: Journal of Abnormal and Social Psychology 62/1961, S. 265-274; James O. Whittaker / Robert D. Meade: Retention of Opinion Change as a Function of Differential Source Credibility. A Cross-Cultural Study, in: International Journal of Psychology 3/1968, S. 103-108; Gary I. Schulman / Chrysoula Worrall: Salience patterns, Source Credibility, and the Sleeper Effect, in: Public Opinion Quarterly 34/1970, S. 371-382.

210 Vgl. Gillig / Greenwald (1974); Noel Capon / James Hulbert: The Sleeper Effect - An Awakening, in: Public Opinion Quarterly 37/1973, S. 333-358; Thomas D. Cook et al.: History 
das Fehlen von Kontrollgruppen, wodurch nicht ausgeschlossen werden kann, daß sich Meinungsänderungen durch äußere Einflüsse ergeben haben sowie Verzerrungen, die sich durch die Messung selbst ergeben können. ${ }^{211}$ Ihrer Meinung nach sind wegen dieser methodischen Mängel Einschränkungen ebenso angebracht wie wegen der unterschiedlichen Untersuchungs-Designs, die keine Vergleichbarkeit hinsichtlich der verwendeten Quellen, der Themen und der Kanäle der Übermittlung gewährleisten. ${ }^{212}$ Gillig und Greenwald, deren eigene Versuche, den Effekt zu replizieren scheiterten, verweisen darauf, daß die berichteten Meinungsänderungen - abgesehen von der Untersuchung von Hovland, Lumsdaine und Sheffield - in keiner der durchgeführten Studien statistisch signifikant waren. ${ }^{213}$ Kommen Capon und Hulbert nach Sichtung der einschlägigen Experimente noch zu dem Schluß, daß es keine Hinweise auf einen allgemeinen "sleeper effect" gibt, daß das Phänomen aber unter bestimmten Bedingungen und bei bestimmten Teilpopulationen auftreten kann, ${ }^{214}$ so konstatieren Gillig und Greenwald knapp: "In short, if the sleeper effect is alive, we do not know where he is living." ${ }^{215}$

Neuere Befunde widersprechen zumindest dieser letztgenannten Sichtweise: Allen und Stiff nehmen für sich in Anspruch, mit ihrer Metaanalyse die Existenz eines 'sleeper effect' nachzuweisen. ${ }^{216}$ Dabei rufen sie zunächst in Erinnerung,

of the Sleeper Effect: Some Logical Pitfalls in Accepting the Null Hypothesis, in: Psychological Bulletin 86/1979, S. 662-679; Charles L. Gruder et al.: Empirical Tests of the Absolute Sleeper Effect Predicted from the Discounting Cue Hypothesis, in: Journal of Personality and Social Psychology 36/1978, S. 1061-1074.

Vgl. Capon / Hulbert (1973), S. 341.

Vgl. Capon / Hulbert (1973), S. 354.

Vgl. Gillig / Greenwald (1974), S. 132.

214

Vgl. Capon / Hulbert (1973), S. 358.

Vgl. Mike Allen / James B. Stiff: Testing Three Models for the Sleeper Effect, in: Western Journal of Speech Communication 53/1989, S. 411-426. 
daß zur Erklärung des Effekts unterschiedliche Modelle herangezogen werden, deren Annahmen variieren. (Zur Veranschaulichung der Modellunterschiede vgl. Abbildung 1.)

Das 'Traditional Sleeper Model' nimmt an, daß Persuasion eine Funktion von Quellen-Glaubwürdigkeit und Inhalt der Mitteilung ist. Es geht davon aus, daß bei hoher Glaubwürdigkeit die Aufmerksamkeit des Rezipienten auf die Quelle gerichtet ist und der Inhalt der Mitteilung daher nicht verarbeitet wird, während bei niedriger Glaubwürdigkeit die Aufmerksamkeit in erster Linie der Mitteilung gilt. Kurz nach dem Erhalt der Mitteilung wirken sich die Quellencharakteristika noch aus, was dazu führt, daß die Einstellungsänderung größer ist, wenn die Mitteilung von Quellen mit hoher Glaubwürdigkeit präsentiert wird. Dauerhafte Einstellungsänderungen basieren nach dieser Vorstellung aber auf dem Inhalt der Mitteilung. Daher ergibt sich zu einem späteren Meßzeitpunkt eine größere Einstellungsänderung, wenn die Quelle geringe Glaubwürdigkeit aufweist, da dann die Mitteilung verarbeitet werden konnte, während die hohe Glaubwürdigkeit eine dauerhafte Einstellungsänderung verhindert, da sie vom Kommunikationsinhalt ablenkt. ${ }^{217}$

217 Vgl. ebd. S. 413f. Der These von der unterschiedlichen Informationsverarbeitung steht allerdings entgegen, daß Recall-Abfragen in allen Studien ergeben, daß Informationen jeweils gleich gut gelernt werden, egal ob sie von der glaubwürdigen oder unglaubwürdigen Quelle kommen. 
Das 'Forgetting Model' legt demgegenüber nahe, daß Persuasion auf Verstärkung beruht. Mitteilungen von Quellen führen danach zunächst generell zu Einstellungsänderungen in der gewünschten Richtung, wobei glaubwürdige Quellen stärkere Effekte erzielen. Im Zeitverlauf wird, wenn es zu keinen weiteren Verstärkungen kommt, die Einstellungsänderung wieder rückgängig gemacht; die Einstellungen der Rezipienten, die je glaubwürdigen und unglaubwürdigen Quellen ausgesetzt waren, nähern sich einander an, bis sie wieder den ursprünglichen Einstellungen entsprechen. ${ }^{218}$

Wie das traditionelle Modell geht auch das 'Disassociation Model' davon aus, daß Persuasion eine Funktion von Quelle und Inhalt der Mitteilung ist. Auch hier ist für eine dauerhafte Einstellungsänderung der Inhalt der Mitteilung ausschlaggebend. Glaubwürdigkeit wirkt nur kurzfristig, da Quelle und Inhalt nicht über längere Zeit hinweg miteinander in Verbindung gebracht werden. Anders als beim traditionellen Modell führt hier die Mitteilung einer Quelle mit niedriger Glaubwürdigkeit jedoch nicht dazu, daß nach Ablauf eines gewissen Zeitintervalls stärkere Einstellungsänderungen als bei Quellen mit hoher Glaubwürdigkeit zu verzeichnen sind, da in beiden Fällen die gleiche Mitteilung auf die gleiche Weise verarbeitet wird. Zudem verursacht die Quelle mit niedriger Glaubwürdigkeit zum ersten Meßzeitpunkt keinerlei Einstellungsänderungen, da sich die "negativen" Eigenschaften der Quelle und die "positiven" Eigenschaften der Mitteilung gegenseitig aufheben. ${ }^{219}$

Die durchgeführte Metaanalyse, die 20 Experimente beinhaltete, erbrachte eine deutliche Ablehnung der Hypothesen, die sich aus dem traditionellen Modell ergeben: nur in fünf Fällen führte die Präsentation einer Quelle mit niedriger Glaubwürdigkeit zu stärkeren Meinungsänderungen als die Quelle mit hoher Glaubwürdigkeit. Das 'Forgetting Model' konnte bei der Hälfte der Fälle bestätigt werden und scheint damit auch nur bedingt zur Erklärung geeignet. Nur fünf Experimente erfüllten die Voraussetzungen, die das 'Disassociation

$\begin{array}{ll}218 & \text { Vgl. ebd. S. 415f. } \\ 219 & \text { Vgl. ebd. S. 416f. }\end{array}$


Model' für eine Prüfung verlangt (keine anfängliche Einstellungsänderung bei Quellen mit niedriger Glaubwürdigkeit). Vier von diesen fünf Experimenten bestätigten die zugrundeliegenden Hypothesen, so daß dieses Modell als das brauchbarste angesehen wird.

Damit ist der 'sleeper effect' zumindest für einige Fälle nachgewiesen. Künftige Untersuchungen sollten nach Auffassung von Allen/Stiff berücksichtigen, daß die Einschätzung der Glaubwürdigkeit nicht allein auf der Basis der durchgeführten Manipulationen erfolgen sollte, sondern daß vielmehr die tatsächliche Wahrnehmung der Glaubwürdigkeit durch die Versuchspersonen in Betracht zu ziehen ist. Zu bedenken sei ferner, daß auch die Inhalte der Mitteilung vergessen werden können. ${ }^{220}$

Eine Erklärung für die Uneinheitlichkeit der Befunde könnte darin liegen, daß die vorliegenden Modelle zwar Quelle und Inhalt, nicht aber den Rezipienten einbeziehen. Es scheint plausibel anzunehmen, daß der 'sleeper effect' abhängig ist davon, ob ein Rezipient sich bezüglich eines Themas eher quellenoder mitteilungsorientiert verhält. Starke persönliche Betroffenheit ('Involvement') und die damit einhergehende verstärkte Konzentration auf den Inhalt könnte das Vergessen der Quelle begünstigen.

Fraglich ist, ob dem 'sleeper effect' jenseits experimenteller Versuchssituationen überhaupt Relevanz zukommt. Gehört es doch zu seinen Voraussetzungen, daß Rezipienten zu einem Thema ausschließlich eine einzige Information aus einer einzigen Quelle erhalten und dann über Wochen hinweg mit dem Thema nicht mehr befaßt sind - eine Bedingung, deren Erfüllung auch in den Experimenten nicht geprüft werden konnte und die in realen Kommunikationssituationen kaum je gegeben ist. Statt dessen sind Rezipienten häufig mit verschiedenen Informationen aus unterschiedlichen Quellen konfrontiert, so daß ihre Glaubwürdigkeit für die Einschätzung der Mitteilung und unter Umständen bereits für die Selektion Bedeutung erlangt. Ob bei einer

220 Vgl. ebd. S. 421ff. Ergänzend siehe auch Anthony R. Pratkanis et al.: In Search of Reliable Persuasion Effects: III. The Sleeper Effect is Dead. Long Live the Sleeper Effect, in: Journal of Personality and Social Psychology 54/1988, S. 203-218. 
bewußten Zuwendung die Quelle ebenso leicht vergessen wird, ist bislang nicht geprüft, kann aber bezweifelt werden.

\subsection{Kritik an der quellenorientierten Glaubwürdigkeitsforschung}

Trotz der Vielzahl der Studien bleiben im Zusammenhang mit der Glaubwürdigkeit von Quellen und ihrer Wahrnehmung einige Fragen offen. Abstriche hinsichtlich der Verallgemeinerung der Befunde sind insoweit angebracht, als die Versuchspersonen meist Schüler oder Studenten sind; dies gilt allerdings für die überwiegende Anzahl psychologischer und sozialpsychologischer Experimente. Neben den Einschränkungen, die sich hinsichtlich der soziodemographischen Merkmale ergeben, ist zu berücksichtigen, daß es sich in der Regel um "captive audiences" handelt, das heißt, diese Gruppen haben nicht von sich aus entschieden, eine Botschaft zu rezipieren, sondern werden durch die Versuchssituation dazu gezwungen. ${ }^{221}$ Daher gilt, daß "die ausschließliche Untersuchung von Studierenden, wie sie für die Wirkung der Glaubwürdigkeitsvariable konstatiert werden muß, eine ernsthafte Gefährdung der externen Validität darstellt." ${ }^{222}$

Bei der häufig undifferenzierten Betrachtung der Rezipienten fällt darüberhinaus ins Gewicht, daß das Publikum offenbar immer als weitgehend "unwissend" eingestuft wurde. Von einzelnen Ausnahmen abgesehen wurden keinerlei Differenzierungen vorgenommen, etwa im Hinblick darauf, ob es unterschiedliche Glaubwürdigkeitseinstufungen gibt, wenn die Zuhörer über Vorwissen zum Thema verfügen. Denkbar ist, daß für Rezipienten, die bezüglich eines Themas selbst kompetent sind (oder sich auch nur für kompetent

221 Vgl. Gerald R. Miller: Communication and Persuasion Research: Current Problems and Prospects, in: Quarterly Journal of Speech 54/1968, S. 268-276, S. 270.

222 Arno Drinkmann / Norbert Groeben: Metaanalysen für Textwirkungsforschung. Methodologische Varianten und inhaltliche Ergebnisse im Bereich der Persuasionswirkung von Texten, Weinheim 1989, S. 63. 
halten), die Glaubwürdigkeit der Quelle gemindert wird (insbesondere, wenn der Inhalt der Mitteilung vom eigenen Wissen abweicht). Eine weitere Möglichkeit wäre, daß in solchen Fällen die Quelle für die Orientierung geringere Bedeutung hat und in erster Linie die Mitteilung selbst beachtet wird. ${ }^{223}$

Die Inhalte der Mitteilung wurden bisher weitgehend vernachlässigt. Es fehlen Untersuchungen, die die Glaubwürdigkeit der Quelle und die Qualität der Mitteilung gleichermaßen systematisch manipulieren. ${ }^{224}$

Da das primäre Ziel der hier vorgestellten Experimente war, Meinungs- und Einstellungsänderungen nachzuweisen, wurden entsprechend auch nur solche Veränderungen gemessen. Andere mögliche Wirkungen werden nicht in Betracht gezogen; so gibt es etwa keine Hinweise darauf, inwieweit die Glaubwürdigkeit von Quellen dazu beiträgt, auf Rezipientenseite bestehende Einstellungen und Meinungen zu verstärken und zu verfestigen.

Schließlich sind, was die Operationalisierung der verschiedenen Dimensionen anbelangt, bei weitem nicht alle Möglichkeiten ausgeschöpft. Obgleich brauchbare Anhaltspunkte geliefert werden, wie 'Kompetenz', 'Vertrauenswürdigkeit', 'Dynamik', 'Sympathie' und 'Ähnlichkeit' definiert und gemessen werden können, wissen wir wenig darüber, ob und wie Rezipienten etwaige Abstufungen von Kompetenzgraden, Graden der Vertrauenswürdigkeit usw. wahrnehmen. Da es den Forschern darauf ankam, Meinungsänderungen herbeizuführen, wurden jeweils besonders prägnante, drastische Gegensätze konstruiert. Im täglichen Leben wird die Orientierung aber dadurch erschwert, daß wir widersprüchliche Informationen von Quellen erhalten, die ähnliche Kompetenz- oder Vertrauensmerkmale aufweisen: "Für die meisten alltäglichen

223 Lediglich eine Untersuchung nimmt sich dieses Zusammenhangs an und liefert eine Bestätigung dieser Vermutung: Vgl. John R. Basehart / Robert N. Bostrom: Credibility of Source and of Self in Attitude Change, in: Journalism Quarterly 49/1972, S. 742-745. Eine gewisse Bedeutung hat das Vorwissen auch im Rahmen der Forschung zum 'Involvement', allerdings wird es auch hier nicht durchgehend berücksichtigt.

224 Vgl. Greenberg / Miller (1966), S. 135. Obgleich die Forderung nahezu 30 Jahre alt ist, gibt es mit Ausnahme der unter 6d) dargestellten Erkenntnisse wenig Wissen über diesen $\mathrm{Zu}$ sammenhang. 
Interaktionen, in denen die relevanten Persönlichkeitsmerkmale sehr viel geringer ausgeprägt sind, erlauben die vorhandenen Befunde kaum fundierte Schlußfolgerungen." 225

Die Forschung zur Glaubwürdigkeit von Quellen wird dominiert von einem Faktormodell, also von der Vorstellung, daß bestimmte Faktoren oder Dimensionen, die sich jeweils aus einem Bündel von Eigenschaften zusammensetzen, Aufschluß über die Glaubwürdigkeit eines Sprechers geben. Obgleich diese Modellvorstellung auch den klassischen Experimenten zur Persuasionsforschung zugrundeliegt, konzentriert sich die Kritik an diesem Modell vorwiegend auf Studien, die mit Semantischen Differentialen und der Faktorenanalyse operieren. Bemängelt wird, daß die verwendeten Dimensionen - in Anlehnung an Berlo/Lemert/Mertz sind dies meist 'Kompetenz', 'Vertrauenswürdigkeit' und 'Dynamik' - willkürliche, künstliche Konstrukte sind, die in der Wahrnehmung der Rezipienten keine Entsprechung finden: "One cannot assume that any of these rating scales or dimensions are those which listeners actually carry around in their heads and uses as the base of their judgments." ${ }^{226}$ Durch die Verwendung gleicher Skalen oder gleicher Bezeichnungen für Faktoren, denen unterschiedliche Skalen zugrundeliegen, werde ferner eine Übereinstimmung in den Ergebnissen suggeriert, die möglicherweise nicht vorhanden ist; gleichzeitig werden unter Umständen weitere Dimensionen, die ebenfalls eine Rolle spielen könnten, gar nicht in Betracht gezogen. ${ }^{227}$ Zudem stellen die Faktoren teilweise ein Artefakt dar und sind

\footnotetext{
225 Köhnken (1990), S. 128.

226

Gary Cronkhite / Jo Liska: A Critique of Factor Analytic Approaches to the Study of Credibility, in: Communication Monographs 43/1976, S. 91-107, S. 92. Siehe auch dies.: The Judgment of Communicant Acceptability, in: M. E. Roloff / G. R. Miller (Hg.): Persuasion: New Directions in Theory and Research, Newbury Park, CA. 1980, S. 100-139. Auch Wakshlag/Edison (1979: 28) bemängeln, daß die Versuchspersonen die Kriterien, aufgrund derer sie Quellen beurteilen, nicht frei wählen können.

227 Vgl. Cronkhite / Liska (1976), S. 93. Siehe auch Christopher J. S. Tuppen: Dimensions of Communicator Credibility: An Oblique Solution, in: Speech Monographs 41/1974, S. 253 260, S. 254.
} 
abhängig von den verwendeten Adjektivpaaren:

"The principal objection to his [i.e. McCroskeys] procedure is that adjectives taken from research involving a concept such as trustworthiness would tend to cluster together as an artefact of the similarity of items regardless of the stimulus object." 228

Dadurch, daß sich scheinbar immer gleiche Dimensionen ergeben, wird nahegelegt, daß diese allgemeingültig sind. 'Glaubwürdigkeit' wird damit als statisches Konstrukt aufgefaßt. Implizit wird unterstellt, daß sich Glaubwürdigkeit automatisch einstellt, wenn bestimmte Dimensionen positiv besetzt sind, unabhängig davon, welchen Rahmenbedingungen die Kommunikation unterliegt. $^{229}$

So wird etwa vernachlässigt, daß Versuchspersonen aus unterschiedlichen Populationen je andere Beurteilungskriterien heranziehen können (Rater-Scale Interaction) und daß unterschiedliche Quellen nicht ohne weiteres mittels der gleichen Skalen beurteilt werden können (Source-Scale Interaction). ${ }^{230} \mathrm{Hinzu}$ kommen Hinweise, daß die Stabilität der Bewertungsdimensionen in Mitleidenschaft gezogen wird, wenn Quellen sich in verschiedenen Kommunikationssituationen äußern. ${ }^{231}$ Schließlich können im Zeitverlauf bei der

$228 \quad$ Wakshlag / Edison (1979), S. 28.

229 Zur Kritik an dieser Generalisierung siehe etwa E. Scott Baudhuin / Margaret Kis Davis: Scales for the Measurement of Ethos: Another Attempt, in: Speech Monographs 39/1972, S. 296-301; Raymond K. Tucker: Reliability of Semantic Differential Scales: The Role of Factor Analysis, in: Western Speech 35/1971, S. 185-190; Ronald L. Applbaum / Karl W. E. Anatol: Dimensions of Source Credibility: A Test for Reproducibility, in: Speech Monographs 40/1973, S. 231-237. Zur Kritik an Applbaum/Anatol siehe Thomas M. Steinfatt: A Criticism of 'Dimensions of Source Credibility: A Test for Reproducibility', in: Speech Monographs 41/1974, S. 291-294 sowie die Erwiderung: Ronald L. Applbaum / Karl W. E. Anatol: A Rejoinder, in: Speech Monographs 41/1974, S. 295-298.

Vgl. Cronkhite / Liska (1976), S. 97ff.

231 Vgl. Ronald L. Applbaum / Karl W. E. Anatol: The Factor Structure of Source Credibility as a Function of the Speaking Situation, in: Speech Monographs 39/1972, S. 216-222, S. 222 . 
gleichen Person unterschiedliche Bewertungskriterien zur Anwendung kommen. ${ }^{232}$

Während einerseits die Konzentration auf wenige Faktoren beklagt wird, der sich eine Reihe von Forschern verschrieben haben, wird andererseits kritisiert, daß durch zahlreiche Studien die Liste der Faktoren, die zur Glaubwürdigkeit beitragen sollen, stetig verlängert wird. Dies wirft die Frage auf, ob durch eine solche Erweiterung eine Klärung des Konstrukts erreicht wird, oder ob nicht vielmehr mit jedem neuen Faktor die Verwirrung wächst. ${ }^{233}$ Einhergehend mit der Diskussion immer neuer Faktoren ist schließlich eine Ausdehnung des Konzepts der Glaubwürdigkeit, die wenig hilfreich ist:

"First, while ethos and communicator credibility originally were relatively well defined concepts, their meaning has been eroded slowly over the past decade as they have come progressively to refer to more global and diffuse notions of communicator image and affective value." 234

Die Ausweitung und mangelnde Abgrenzung zum umfassenderen Imagebegriff kommt dabei unter Umständen bereits bei der Konstruktion der Skalen des Semantischen Differentials zum Tragen. ${ }^{235}$ Die wachsende Beliebigkeit betrifft nicht nur die Urteilsdimensionen; häufig werden auch die Bewertungsobjekte nicht mehr in ihrer Funktion als 'Quellen' oder 'Kommunikatoren' präsentiert, sondern es wird pauschal gefragt, wie sie 'als Person' zu beurteilen sind: "The implicit expansion of the credibility concept making it synonymous with

232 Vgl. F. C. Powell / J. W. Wanzenried: Perceptual Changes in Source Credibility: Repeated Tests Using Two Candidates During a Political Campaign, in: Perceptual and Motor Skills 73/1991, S. 1107-1114.

233 Vgl. Infante / Rancer / Womack (1993), S. 189f.

234 Jesse G. Delia: A Constructivist Analysis of the Concept of Credibility, in: Quarterly Journal of Speech 62/1976, S. 361-375, S. 364.

235 Dies trifft auf die Konstruktion der Skalen von Berlo/Lemert/Mertz zu, die zu den am häufigsten verwendeten gehört. Hier wurde, wie in Abschnitt C.3 (S. 45ff.) erläutert, die Akzeptanz von Quellen zugrundegelegt. 
general person perception seems now to be the rule rather than the exception." ${ }^{236}$

\subsection{Alternative Modellvorstellungen}

Unabhängig von der Anzahl der zugrundegelegten Faktoren besteht ein Einwand gegenüber dem Faktormodell darin, daß es keinen Aufschluß darüber gibt, ob Rezipienten tatsächlich alle Faktoren benutzen, um einer Quelle Glaubwürdigkeit zuzuschreiben. Wie oben dargelegt, deutet eine Reihe von Befunden darauf hin, daß, je nachdem welche Quelle in welcher Situation spricht, einzelne Charakteristika mehr oder weniger bedeutsam sind. $\mathrm{Zu}$ berücksichtigen ist schließlich auch, daß nicht alle Rezipienten die gleichen Beurteilungskriterien zugrundelegen. Aus diesen Überlegungen entstanden zwei Modelle, die ihr Augenmerk darauf richten, die Rezipienten und ihre Bedürfnisse sowie die Kommunikationssituation verstärkt in die Betrachtung einzubeziehen. $^{237}$

Vertreter des funktionalen Modells konzedieren zwar, daß die herkömmlichen Glaubwürdigkeits-Skalen zumindest potentielle Beurteilungskriterien sein können, aber welche Merkmale tatsächlich als Kriterien dienen und welche bloße Wahrnehmungen darstellen, die für die Beurteilung keine Rolle spielen, ist abhängig davon, welche Funktion die Quelle für den Rezipienten in der aktuellen Kommunikationssituation einnimmt. ${ }^{238}$ Die Kriterien, die verwendet werden, um die Quelle zu beurteilen, werden aus den aktuellen Bedürfnissen

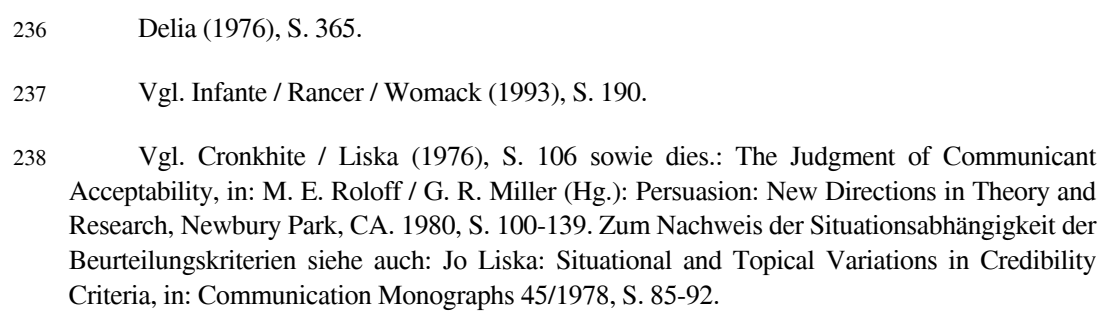
Acceptability, in: M. E. Roloff / G. R. Miller (Hg.): Persuasion: New Directions in Theory and Research, Newbury Park, CA. 1980, S. 100-139. Zum Nachweis der Situationsabhängigkeit der Beurteilungskriterien siehe auch: Jo Liska: Situational and Topical Variations in Credibility Criteria, in: Communication Monographs 45/1978, S. 85-92. 
und Zielen des Rezipienten in der jeweiligen Kommunikationssituation abgeleitet. Je mehr die Bedürfnisse durch die Quelle befriedigt werden, desto glaubwürdiger ist sie. ${ }^{239}$ Glaubwürdigkeit wird dabei ausschließlich als Fähigkeit der Quelle betrachtet, beim Rezipienten Meinungs- und Verhaltensänderungen auszulösen. ${ }^{240}$

Das funktionale Modell setzt sich aus fünf Komponenten zusammen. Einbezogen werden: (1) (physisch wahrnehmbare) Merkmale von Quelle und Mitteilung, die als Stimulus dienen - dazu gehören Aussehen, Stimme und Sprechverhalten ebenso wie Sprache, Argumentation und Aufbau der Mitteilung; (2) abgeleitete Charakteristika der Quelle - etwa Bildungsstand, Intelligenz oder Absichten - die auf Schlußfolgerungen des Rezipienten beruhen; (3) Funktionen, die die Quelle für den Empfänger in einer bestimmten Situation hat; (4) Kriterien, aufgrund derer der Rezipient die Quelle für geeignet hält, eine bestimmte Funktion auszufüllen; und (5) Veränderungen im Wissen und Verhalten beim Empfänger. Dabei können sich im Verlauf der Kommunikation alle Elemente gegenseitig beeinflussen. ${ }^{241}$

Der statischen Betrachtungsweise des Faktormodells setzt Delia einen konstruktivistischen Ansatz entgegen:

"To obtain a satisfactory understanding of ethos as a process we must ask and answer the fundamental question: 'By what means, that is by what perceptual or judgmental processes, does a receiver come to a particular credibility evaluation of a communicator?' Or, 'In the actual encounter between communicator and receiver, how is it that the receiver translates aspects of the communicator's appearance, behavior, and assertions into judgments concerning his credibility?"' 242

239 Vgl. Dominic A. Infante et al.: A Comparison of Factor and Functional Approaches to Source Credibility, in: Communication Quarterly 31/1983, S. 43-48, S. 44.

Vgl. Cronkhite / Liska (1976), S. 104.

Vgl. Cronkhite / Liska (1976), S. 104f.

Delia (1976), S. 366 
Ausgangspunkt dieses Ansatzes ist die Überlegung, daß Wahrnehmung mit selektiver Aufmerksamkeit gegenüber verschiedenen Aspekten der Umwelt einhergeht, wobei die wahrgenommenen Reize im Rahmen von Kategorien oder Konstrukten interpretiert werden, die auf (individuellen) Erwartungen und Vorerfahrungen basieren. ${ }^{243}$

Bei der Eindrucksbildung von Personen entwickelt nach dieser Vorstellung mithin jeder Beobachter eine "implizite Persönlichkeitstheorie", wobei er sich auf ein System von Konstrukten stützt, die seinen Wahrnehmungsrahmen bilden. Das wahrgenommene Verhalten und die äußere Erscheinung einer Person sowie ihre Äußerungen werden aufgrund dieser interpersonalen Konstrukte geordnet. Die Konstrukte selbst können als bipolare Bewertungspaare aufgefaßt werden, die ein Kontinuum bilden. Die Bewertungsdimensionen können von kulturellen Normen determiniert sein oder vom Individuum definiert werden. ${ }^{244}$

Glaubwürdigkeit wird verstanden als eigenständiger Aspekt im Konstruktionsprozeß interpersonaler Wahrnehmung und kann nur in Verbindung mit Kommunikationssituation und -kontext bestimmt werden. Für unterschiedliche Situationen und Kontexte werden dabei vom Rezipienten je unterschiedliche Konstrukte für die Eindrucksbildung zugrundegelegt. ${ }^{245}$ Allgemeingültige

243 Vgl. Delia (1976), S. 367 sowie Siegfried J. Schmidt: Die Wirklichkeit des Beobachters, in: Klaus Merten / Siegfried J. Schmidt / Siegfried Weischenberg (Hg.): Die Wirklichkeit der Medien. Eine Einführung in die Kommunikationswissenschaft, Opladen 1994, S. 3-19, S. $8 \mathrm{ff}$.

244 Vgl. Delia (1976), S. 368; Infante / Rancer / Womack (1993), S. 190; sowie ausführlich: George A. Kelly: The Psychology of Personal Constructs, 2 Vols., New York 1955.

245 Demonstriert wird dies etwa in einem Versuch von Jesse G. Delia et al.: The Dependency of Interpersonal Evaluations on Context Relevant Beliefs about the Other, in: Communication Monographs 42/1975, S. 10-19: Die Versuchspersonen erhielten Informationen über einen Studenten, die sein Verhalten im gesellschaftlichen Umgang und im Arbeitsleben betrafen. Dabei war wechselweise einer der Kontexte mit positiven und einer mit negativen Beschreibungen wiedergegeben, die angeblich von Personen stammten, die den Betreffenden gut kannten. Die Versuchspersonen wurden instruiert, sich vorzustellen, daß einer ihrer Freunde diesen Studenten a) auf informeller, privater Ebene oder b) zur Erledigung einer gemeinsamen Aufgabe treffen sollte und sie diesem Freund mangels anderer Informationen ihre eigenen Eindrücke schildern sollten. Es zeigte sich, daß die abgegebenen Schilderungen klar am 
Glaubwürdigkeits-Faktoren oder -Funktionen kann es nach dieser Auffassung nicht geben. Daher verbietet sich für die Erforschung auch die Vorlage von Adjektivlisten oder Semantischen Differentialen, da die Rezipienten hier gezwungen werden, Stellungnahmen zu Dimensionen abzugeben, die sie selbst gar nicht zur Beurteilung verwenden würden. Statt dessen werden Versuchspersonen gebeten, ihre Eindrücke niederzuschreiben. Durch einen Vergleich solcher Eindrücke ergeben sich Rückschlüsse auf typische Konstruktionsmuster in verschiedenen Situationen. ${ }^{246}$

Beiden Ansätzen ist gemeinsam, daß sie eine stärkere Orientierung an den Vorstellungen des Rezipienten und eine Einbeziehung der Kommunikationssituation fordern, um Aufschluß über die Bedingungen der Zuschreibung von Glaubwürdigkeit zu erlangen. Beide betonen den Prozeßcharakter der Kommunikation und verweisen darauf, daß die Eindrucksbildung im Verlauf der Kommunikation Veränderungen unterworfen sein kann. ${ }^{247}$ Beide wollen sich mit ihrer Forderung vom Faktormodell der Glaubwürdigkeit abgrenzen, wobei sie das Faktormodell auf Studien eingrenzen, die Faktorenanalysen durchführen.

Der Schwerpunkt der Betrachtung liegt bei Vertretern des funktionalen Ansatzes auf den Bedürfnissen der Rezipienten, die sich in Abhängigkeit von der Kommunikationssituation bilden. Ihnen ist eher daran gelegen, das Faktormodell zu ergänzen; sie gehen davon aus, daß es durchaus Charakteristika der Quelle gibt, die situationsübergreifend für das Zustandekommen von

jeweiligen Kontext orientiert waren: War etwa vorgegeben, daß die beiden gemeinsam arbeiten sollten, wurden überwiegend die (positiven oder negativen) Merkmale, die den Studenten als Mitarbeiter kennzeichneten, weitergegeben und die anderen vernachlässigt. Jesse G. Delia / Barbara J. O'Keefe / Daniel J. O'Keefe: The Constructivist Approach to Communication, in: F. E. X. Dancer (Hrsg.): Human Communication Theory, New York 1982, S. 147-191. 
Glaubwürdigkeit verantwortlich sind (z.B. Ehrlichkeit). ${ }^{248}$ Wie beim Faktormodell ist auch beim funktionalen Modell das Ziel, generalisierbare Aussagen über das Zustandekommen von Glaubwürdigkeit zu treffen; geändert hat sich lediglich die Perspektive der Betrachtung: Um diese allgemeingültigen Charakteristika zu vervollständigen, ist eine Verlagerung des Forschungsinteresses von "typischen Quellen" auf "typische Situationen" und "typische Bedürfnisse" nötig.

In dieser Zielvorstellung liegt der Hauptunterschied zum konstruktivistischen Modell, das allgemeingültige Faktoren und Funktionen ablehnt und sich statt dessen auf die Suche nach typischen Konstruktionsmustern begibt, die Rezipienten bei der Einschätzung von Quellen verwenden. ${ }^{249}$ Im Zentrum der Aufmerksamkeit stehen hier also der Rezipient und seine individuellen Konstrukte. Die selektive Betrachtungsweise, die seiner Konstruktion zugrundeliegt, ist nicht nur an Bedürfnissen ausgerichtet, sondern auch an früheren Erfahrungen und allgemeinen oder speziellen Erwartungen. Daher können zwei Rezipienten in der gleichen Kommunikationssituation zu gänzlich unterschiedlichen Einschätzungen gelangen. Perloff kritisiert diese Auffassung:

"Although context influences credibility judgments, we should not push context effects too far. Although individuals do use different criteria to evaluate speakers in different situations, they also use some criteria more than others. Expertise and trustworthiness are apt to be highly salient criteria for most message recipients." 250

Die Beständigkeit, mit der hauptsächlich diese beiden Dimensionen in den verschiedenen Experimenten immer wieder bestätigt wurden, spricht für eine solche, integrative Sichtweise, wie sie auch Vertretern des funktionalen Ansatzes zu eigen ist. Bei einer Verlagerung des Forschungsinteresses auf

\footnotetext{
248 Vgl. Liska (1978), S. 90f.; Cronkhite / Liska (1976), S. 106.

249 Vgl. Infante / Rancer / Womack (1993), S. 192.

250 Richard M. Perloff: The Dynamics of Persuasion, Hilsdale N.J. 1993, S. 141.
} 
Rezipienten und Kommunikationssituationen sollten die wahrgenommenen Quellencharakteristika nicht außer Acht gelassen werden. Vielmehr ist davon auszugehen, daß die bisher ermittelten Dimensionen - für unterschiedliche Individuen in unterschiedlichen Kontexten - jeweils unterschiedliche Relevanz besitzen. Eine verstärkte Orientierung an Rezipienten und Kontexten könnte darüberhinaus dazu führen, daß sich weitere Dimensionen ergeben, die in den Augen der Betrachter zur Glaubwürdigkeit einer Quelle beitragen, bisher aber noch nicht berücksichtigt wurden.

\subsection{Zwischenbilanz}

Bei der Erforschung der Frage, wie Meinungs- und Einstellungsänderungen zustandekommen, rückte zunächst das 'Prestige' eines Kommunikators sowie seit Beginn der 40er Jahre die Glaubwürdigkeit der Quelle einer Aussage als ein zentrales Kriterium in den Blickpunkt. Seither gehört sie zu den am meisten untersuchten Aspekten der Persuasion; eine Metaanalyse zeigt, daß eine hohe Glaubwürdigkeit der Quelle nahezu durchweg zu vermehrten Meinungs- und Einstellungsänderungen führt. ${ }^{251}$

Glaubwürdigkeit ist eine Eigenschaft, die eine Quelle nicht von sich aus besitzt, sondern die ihr von ihren Rezipienten zugeschrieben wird. Bei dieser Zuschreibung stützen sich Rezipienten auf eine Reihe von Merkmalen, die in verschiedenen Kommunikationssituationen unterschiedlichen Stellenwert haben. Am häufigsten gründet sich die Einschätzung der Glaubwürdigkeit einer Quelle auf die beiden Dimensionen Kompetenz und Vertrauenswürdigkeit. Kompetenz manifestiert sich im Ausmaß des Wissens um ein bestimmtes Fachgebiet, in Hinweisen auf Qualifikation, Erfahrung, Leistungen oder Intelligenz sowie im Innehaben von Führungspositionen. Die Zuschreibung von Vertrauenswürdigkeit steht in engem Zusammenhang mit der Ehrlichkeit

251 Vgl. Drinkmann / Groeben (1989), S. 67ff. 
und Aufrichtigkeit einer Quelle einerseits und ihrer Unparteilichkeit, Unabhängigkeit und Selbstlosigkeit andererseits. Bedeutsam ist zudem der Aspekt der Zuverlässigkeit und die Übereinstimmung von Reden und Handeln.

Auch die Dynamik der Quelle, die sowohl durch Aktivität und Stärke als auch durch verschiedene Aspekte des Sprechverhaltens gekennzeichnet ist, spielt eine Rolle, wobei umstritten ist, ob dieser Faktor als eigenständige Komponente aufzufassen ist oder ob er eher additive Funktion hat. Ähnlichkeiten zwischen Quelle und Rezipient hinsichtlich der Gruppenzugehörigkeit oder hinsichtlich Einstellungen und Werthaltungen können ebenso zur Glaubwürdigkeit einer Quelle beitragen wie Sympathie oder physische Attraktivität. Die letztgenannten haben eher den Status von Ersatzkriterien, die herangezogen werden, wenn eindeutigere Hinweise zur Einschätzung der Quelle fehlen. Schließlich dient auch das nonverbale und extralinguistische Sprechverhalten dazu, die Glaubwürdigkeit einer Quelle zu bestimmen. Keine allgemeingültigen Aussagen bezüglich der Glaubwürdigkeit läßt das Geschlecht des Sprechers zu. Es gibt Hinweise, daß die Einschätzung sowohl abhängig vom geschlechtsspezifischen Sprechverhalten als auch vom Thema und von der Einstellung der Rezipienten Frauen gegenüber ist.

Diese Dimensionen, die ursächlich am Zustandekommen von Glaubwürdigkeit beteiligt sind, sind nicht voneinander unabhängig, sondern beeinflussen sich in unterschiedlichem Ausmaß gegenseitig. Wahrgenommene Ähnlichkeiten zwischen Quelle und Rezipient können dazu beitragen, daß die Quelle für sympathischer gehalten wird; umgekehrt wird eine sympathische Quelle vom Rezipienten oft als ihm ähnlicher wahrgenommen. Nicht generell, aber unter bestimmten Konstellationen wirken sich Ähnlichkeiten auch auf die Einschätzung von Kompetenz und einzelne Komponenten der Vertrauenswürdigkeit aus. Zugeschriebene Kompetenz führt manchmal dazu, daß eine Quelle als vertrauenswürdiger wahrgenommen wird. Attraktivität führt - wie eine Reihe von Studien nahezu einhellig zeigt - dazu, daß eine Quelle als sympathischer, nicht aber als kompetenter oder vertrauenswürdiger eingestuft wird. Ferner ist ein Zusammenhang der Attraktivität mit der Dimension 'Dynamik' 
nachgewiesen. Letztere steht auch in Wechselwirkung mit dem Sprechverhalten. Zusätzlich beeinflussen Störungen im Sprechverhalten sowie die Sprechgeschwindigkeit die Einschätzung der Kompetenz eines Sprechers; nonverbales Verhalten, Stimmeigenschaften und die Art des Vortrags können sich auf die wahrgenommene Vertrauenswürdigkeit und Sympathie auswirken.

Den genannten Eigenschaften von Quellen wurde größte Aufmerksamkeit zuteil; dagegen wurde häufig nicht berücksichtigt, daß die beiden anderen Strukturelemente jedes Kommunikationsvorgangs, der Rezipient und die Mitteilung, ebenfalls einen Beitrag zur Wahrnehmung von Glaubwürdigkeit leisten. Erst seit Ende der 60er Jahre werden individuelle Unterschiede in der Wahrnehmung auf seiten der Rezipienten berücksichtigt und genauer betrachtet, allerdings nicht kontinuierlich und systematisch in die Forschungsdesigns integriert. Nicht hinreichend geklärt ist, inwieweit persönlichkeitsspezifische Eigenschaften (wie etwa Selbstbewußtsein oder Autoritätsgläubigkeit) die Zuschreibung von Glaubwürdigkeit fördern oder behindern. Dagegen konnte gezeigt werden, daß die Relevanz eines Themas für den Rezipienten ('Involvement') unterschiedliche Verarbeitungsstrategien nach sich zieht, wobei die Glaubwürdigkeit der Quelle unterschiedlichen Stellenwert hat: Wenn das Thema wichtig für den Rezipienten ist und er über Vorwissen bezüglich des Themas verfügt und/oder Interesse aufbringt, konzentriert er sich in erster Linie auf den Inhalt der Mitteilung; bei Themen, die für ihn wenig bedeutsam sind oder über die er wenig weiß, orientiert er sich vorwiegend an der Quelle und ihren Glaubwürdigkeitsmerkmalen.

Unabhängig vom Grad der Involviertheit kann auch der Zeitpunkt der Information über die Quelle für die Orientierung verantwortlich sein: Wird die Quelle erst nach der Rezeption der Mitteilung identifiziert, stellt die Mitteilung den primären Orientierungsrahmen dar; erfolgt die Quellenidentifikation am Anfang, beeinflußt sie die Rezeption der nachfolgenden Mitteilung.

Nur wenig gesicherte Erkenntnisse geben Aufschluß darüber, wie sich Aufbau, Stil und Inhalt der Mitteilung auf die Glaubwürdigkeit der Quelle auswirken. Die Verwendung von Metaphern und Vergleichen kann ebenso 
positive Effekte nach sich ziehen wie die Verwendung von Fachtermini, ein abwechslungsreiches Vokabular oder personalisierende Elemente. Die genannten Stilmittel sind besonders geeignet, die Wahrnehmung der Kompetenz der Quelle zu stärken. Der Einsatz von Humor als Stilmittel erbringt widersprüchliche Befunde. Zu einer Stärkung der Glaubwürdigkeit trägt auch das Vorlegen von Beweismaterial bei, selbst wenn die zitierten Quellen nicht transparent gemacht werden. Die Offenlegung von zitierten Quellen, die ihrerseits Glaubwürdigkeitsmerkmale aufweisen, kann die Vertrauenswürdigkeit der zitierenden Quelle untermauern - die Wechselwirkungen sind allerdings zu wenig erforscht, um hier eindeutige Aussagen zu treffen.

Daß Rezipienten und Kommunikationsinhalte nicht in gleichem Maße wie die Quellen von der Forschung berücksichtigt wurden, stellt gleichzeitig einen der wesentlichen Kritikpunkte dar. Um die Forschungsdefizite zu markieren, die den Rezipienten betreffen, wurden mit dem funktionalen und dem konstruktivistischen Ansatz zwei Modellvorstellungen präsentiert, die das herkömmliche Faktormodell ergänzen bzw. ablösen sollten. Die Vorschläge für eine empirische Umsetzung der Modelle blieben spärlich. ${ }^{252}$

Bemängelt werden ferner methodische Aspekte, insbesondere die Beschränkung auf studentische Versuchspersonen und die undifferenzierte Anwendung der Faktorenanalyse. Schließlich geben die bei der Operationalisierung konstruierten, meist recht drastischen Gegensätze zwar Aufschluß darüber, welche Dimensionen sich bei der Zuschreibung von Glaubwürdigkeit auswirken können; sie verraten jedoch kaum etwas darüber, welches Gewicht weniger massiven Hinweisen, die alltagsnäher sind, zukommt. Weitgehend ungewiß bleibt auch, welchen Einfluß das Zusammenwirken verschiedener Dimensionen hat, die unter Umständen teils 'positive' und teils 'negative' Kriterien liefern.

252 Vgl. Liska (1978) und Infante et al. (1983) für den funktionalen Ansatz sowie Delia et al. (1975) für den konstruktivistischen Ansatz, wobei bei letzteren nur die Eindrucksbildung allgemein, nicht aber Glaubwürdigkeit geprüft wird. 


\title{
6 "Am glaubwürdigsten im Fernsehen ist immer noch 'Das Wort zum Sonntag'!" Die Glaubwürdigkeit des Mediums
}

\author{
"Glaubwürdigkeit ist die erste Tugend, die eine Nachrichtensendung \\ aufweisen muß, wenn sie langfristig Erfolg haben will." ${ }^{2}$
}

Als eine besondere Art von Quellen können schließlich Massenmedien betrachtet werden. Vereinzelt wird hier eine "Glaubwürdigkeits-Kluft" oder gar ein "Glaubwürdigkeits-Canyon" konstatiert, womit gemeint ist, daß die Rezipienten den Medien immer weniger Vertrauen entgegenbringen. ${ }^{3}$ Laut Gaziano ist es aber übertrieben, von einer Glaubwürdigkeitskrise zu sprechen, da Medien - mit einigen Abstrichen - vom Publikum weitgehend positiv bewertet werden. ${ }^{4}$

\subsection{Eindimensionale Ansätze}

Daß unterschiedliche Medienarten unterschiedliche Glaubwürdigkeit aufweisen können, wurde verstärkt thematisiert, seit das Roper-Umfrage-Institut 1959 erstmals die Frage stellte:

Bodo Hauser, Moderator des Magazins 'Frontal' (ZDF), im Anschluß an einen Beitrag über gefälschte Fernseh-Reportagen; zitiert nach: TV-Spielfilm 6/1996, S. 7.

2

Ulrich Wickert: Eine Frage der Qualität. Aktuelle Informationen im Ersten, in: Arbeitsgemeinschaft der öffentlich-rechtlichen Rundfunkanstalten der Bundesrepublik Deutschland (ARD) (Hrsg.): ARD Jahrbuch 1995, Hamburg 1995, S. 120-123, S. 122.

3

Vgl. Robert L. Bishop / Sue A. Schultz: The Credibility Gap: Is It Widening? in: Journalism Quarterly 44/1967, S. 740-741; Robert L. Bishop / Mary Boersma / John Williams: Teenagers and News Media: Credibility Canyon, in: Journalism Quarterly 46/1969, S. 597-599.

4

Vgl. Cecilie Gaziano: How Credible Is the Credibility Crisis? in: Journalism Quarterly 65/1988, S. 267-278, 375, S. 278. 
"If you got conflicting or different reports of the same news story from radio, television, the magazines and the newspapers, which of the four versions would you be most inclined to believe - the one on radio or television or magazines or newspapers?" ${ }^{5}$

Diese Vorgehensweise der Roper-Umfragen wurde in zahlreichen Untersuchungen, zum Teil mit geringfügigen Variationen, übernommen. ${ }^{6}$ Übereinstimmend erbringt diese Fragestellung seit den 60er Jahren jeweils einen hohen Glaubwürdigkeitsvorsprung des Fernsehens gegenüber der Tageszeitung; Zeitschriften und Hörfunk landen meist abgeschlagen auf hinteren Plätzen. ${ }^{7}$ Die dem Fernsehen zugeschriebene Glaubwürdigkeit nimmt dabei über die Jahre hinweg zu, während die Werte für die Zeitung nahezu konstant bleiben und die für den Hörfunk sinken. ${ }^{8}$ Dies läßt vermuten, daß die Einschätzung mit veränderten Nutzungsgewohnheiten zusammenhängt, wobei die Art der Kausalbeziehung zunächst noch offen ist: Wird Fernsehen häufiger genutzt, weil es für glaubwürdiger gehalten wird, oder wird es für glaubwürdiger gehalten, weil es häufiger genutzt wird und damit dem Rezipienten vertrauter ist? Daß ein Zusammenhang besteht, legen die Ergebnisse der jeweils

5 Burns W. Roper: Changing Public Attitudes toward Television and Other Media 19591976, in: Communications 4/1978, S. 220-238, S. 222.

6

Vgl. zum Beispiel Leslie A. Baxter / John R. Bittner: High School and College Students Perceptions of Media Credibility, in: Journalism Quarterly 51/1974, S. 517-520; Ronald Mulder: Media Credibility: A Use-Gratifications Approach, in: Journalism Quarterly 57/1980, S. 474-477; Joey Reagan / Jayne Zenaty: Local News Credibility: Newspapers vs. TV Revisited, in: Journalism Quarterly 56/1979, S. 168-172.

Seit Beginn der 70er Jahre wird das Fernsehen laut Roper (1978: 223) kontinuierlich von über 50 Prozent der Bevölkerung als das glaubwürdigste Medium angesehen. Tageszeitungen weisen Werte zwischen 20 und 24 Prozent auf, Hörfunk und Zeitschriften liegen um 8 Prozent. Die Anzahl der Befragten, die eine solche Einschätzung nicht vornehmen konnten oder wollten, sank von rund 17 Prozent in den Anfangsjahren auf rund 10 Prozent. (Vgl. dazu auch die Verlaufsgraphik bei Bentele(1988), S. 411.)

Die Tatsache, daß der Zuwachs der Glaubwürdigkeit des Fernsehens nicht auf Kosten der Zeitung erfolgt, wird dabei häufig übersehen. Vgl. John Newhagen / Clifford Nass: Differential Criteria for Evaluating Credibility of Newspapers and TV News, in: Journalism Quarterly 66/1989, S. 277-284, S. 284. 
parallel gestellten Frage nach der Nutzung der Medien nahe. Das Fernsehen löst hier erstmals 1964 die Zeitung als meistgenutztes Medium ab und behauptet seither die Spitzenposition; die Bedeutung des Hörfunks als Nachrichtenmedium sinkt. ${ }^{9}$ Allerdings gibt es Hinweise, daß dieser Zusammenhang vorwiegend durch die Art der Fragestellung entsteht: Eine Sekundäranalyse zeigt, daß sich schwache Korrelationen lediglich für die eher affektive Roper-Frage nach der Präferenz ergeben. Fragt man nach dem tatsächlichen Nutzungsverhalten (etwa nach dem Zeitaufwand oder den am Vortag genutzten Medien), ergibt sich keine Beziehung von Häufigkeit der Nutzung und Glaubwürdigkeit. ${ }^{10}$

Die Frage nach den Gründen für die unterschiedliche Glaubwürdigkeit von Medien wird nicht allzuoft gestellt. Wo dies doch geschieht, ergibt sich als Erklärung für den Glaubwürdigkeitsvorsprung des Fernsehens die optische Komponente ("seeing is believing") sowie die erhöhte Aktualität und das LiveErlebnis. ${ }^{11}$ Ferner wird von Befragten das "bessere Personal" des Fernsehens

Vgl. Roper (1978), S. 221. Die Frage im Wortlaut: "First, I'd like to ask you where you usually get most of your news about what's going on in the world today - from the newspapers or radio or television or magazines or talking to people or where?" Ein positiver Zusammenhang von Nutzung und Glaubwürdigkeit wird ferner konstatiert bei Bruce H. Westley / Werner J. Severin: Some Correlates of Media Credibility, in: Journalism Quarterly 41/1964, S. 325-335; Richard F. Carter / Bradley S. Greenberg: Newspaper or TV: Which Do You Believe? in: Journalism Quarterly 42/1965, S. 29-34; Bradley S. Greenberg: Media Use and Believability: Some Multiple Correlates, in: Journalism Quarterly 43/1966, S. 665-670; Eugene F. Shaw: Media Credibility: Taking The Measure of a Measure, in: Journalism Quarterly 50/1973, S. 306311.

Vgl. Tony Rimmer / David Weaver: Different Questions, Different Answers? Media Use and Media Credibility, in: Journalism Quarterly 64/1987, S. 28-36, 44.

11

Vgl. Cecilie Gaziano / Kristin McGrath: Measuring the Concept of Credibility, in: Journalism Quarterly 63/1986, S. 451-462, S. 456 sowie: Lawrence K. H. Chang / James B. Lemert: The Invisible Newsman and Other Factors in Media Competition, in: Journalism Quarterly 45/1968, S. 436-444. Zur Überlegenheit der audiovisuellen Präsentation in bestimmten Bereichen siehe auch William K. Ripley: Medium of Presentation: Does It Make a Difference in the Reception of Evaluation Information? in: Educational Evaluation and Policy Analysis 7/1985, S. 417-425. Obgleich die Einschätzungen der Glaubwürdigkeit für die Quelle nicht durchweg niedriger sind, wenn ihre Aussage in schriftlicher Form vorliegt, ergeben sich 
ins Feld geführt. Es konnte jedoch gezeigt werden, daß diese Einschätzung beim Publikum nicht auf tatsächlich wahrgenommene Qualitätsunterschiede zurückzuführen ist, sondern ausschließlich auf die Bekanntheit der Präsentatoren: Während Zeitungsjournalisten "unsichtbar" bleiben, wird ihren Kollegen beim Fernsehen alleine wegen ihrer häufigen optischen Präsenz das Prädikat "besser" verliehen. ${ }^{12}$ Neben diesen Unterschieden, die in der Präsentation begründet sind, werden weitere Faktoren zur Erklärung herangezogen:

"1. Since newspapers were more given to controversy, they suffered the fate of the messenger who brought bad news.

2. Political bias has infected printed media; most reporters are liberals.

3. The broadcast media were by government regulation apolitical." ${ }^{13}$

Die Stichhaltigkeit dieser Argumente wird von Edelstein jedoch bezweifelt: Kontroverse Berichterstattung fände sich ebenso in Fernsehnachrichten; Fernsehanstalten stünden ihrer politischen Ausrichtung nach weiter links als die meisten Zeitungen, und der Einfluß von Politikern auf das Fernsehen werde für weit größer erachtet als der, den sie auf Printmedien ausüben. ${ }^{14}$

Die Vorgehensweise der Roper-Untersuchungen wird in der Folge von vielen Seiten kritisiert. Einer der Einwände gegen das Verfahren ist die mangelnde Realitätsnähe der Frage. Die Argumentation lautet dahingehend, daß widersprüchliche Nachrichten nicht häufig vorkommen und daß zusätzlich unklar ist, ob sich die Rezipienten einer etwaigen Widersprüchlichkeit bewußt sind. Außerdem müßten die widersprüchlichen Nachrichten ungefähr gleichzeitig auftreten, was ebenfalls unrealistisch ist. ${ }^{15}$

im Vergleich mit der Präsentation via Bildschirm Defizite in einer zentralen Dimension (Objektivität). (Vgl. S. 422ff.) communications Review 2/1974, S. 48-52, S. 49. Siehe auch Walter Gantz: The Influence of 
Ähnlich äußern sich zunächst Gaziano/McGrath: "Although this question has been pursued for about a quarter of a century, apparently no researchers have asked people if they ever actually have received conflicting news stories about different media." ${ }^{16}$ Im folgenden können sie allerdings nachweisen, daß etwa die Hälfte ihrer Befragten schon einmal widersprüchliche Meldungen unterschiedlicher Medien wahrgenommen hatte und daß ein großer Teil von diesen niedrigere Werte bei der Glaubwürdigkeitseinschätzung aufweist. ${ }^{17}$

Ein weiterer Vorwurf lautet, daß die Frage in dieser Form zu allgemein gestellt ist: Es bleibt unklar, was sich die Befragten jeweils unter 'Zeitung' und 'Fernsehen' vorstellen. Diesen Umstand bezeichnet Edelstein als "matter of basic source identification":

"Was the individual referring to the characteristics of the channel itself; to characteristics of content; to an institutionalized source image, such as the newspaper or television station itself; to a bylined author of an article or report; or to a news source within the story?" 18

Es ist wohl auszuschließen, daß die Befragten einzelne Primärquellen von Nachrichten vor Augen haben, wenn sie gebeten werden, zur Glaubwürdigkeit einer Medienart Stellung zu nehmen, da die Primärquellen in der Regel nicht exklusiv an ein Medium gebunden sind. In Betracht zu ziehen ist jedoch, daß mancher bei der Beantwortung der Frage einen bestimmten Sender oder eine bestimmte Zeitung vor Augen hat. So wurde zum Beispiel vermutet, daß die Einschätzungen der Befragten sich bei der Roper-Frage auf die Programme der 'National Networks' einerseits und der lokalen Tageszeitungen andererseits beziehen, was einer "mangoes and zucchini comparison" ${ }^{19}$ gleichkommt.

Researcher Methods on Television and Newspaper News Credibility Evaluations, in: Journal of Broadcasting 25/1981, S. 155-169, S. 156.

16 Gaziano / McGrath (1986), S. 452.

$17 \quad$ Vgl. ebd. S. 456.

$18 \quad$ Edelstein (1973), S. 12.

19 Bradley S. Greenberg / Michael E. Roloff: Mass Media Credibility: Research Results and Critical Issues, in: News Research Bulletin No. 6 (Washington, D.C.: American Newspaper 
Diesen Vorwurf nahmen eine Reihe von Forschern zum Anlaß, um ausdrücklich nach der Glaubwürdigkeit von lokalen Medien zu fragen. Auch im direkten Vergleich schneidet lokales Fernsehen gegenüber Lokalzeitungen hinsichtlich der Glaubwürdigkeit besser ab. ${ }^{20}$ Die eigene Lokalzeitung wird aber hinsichtlich ihrer Fairneß besser beurteilt als Zeitungen allgemein. ${ }^{21}$

Fragt man gezielt nach einzelnen Medien oder Medienvertretern, verringert sich die Distanz zwischen Fernsehen, Hörfunk und Zeitungen:

"To a degree, the Roper issue - and the Roper thesis - presupposes a classification of news media that doesn't conform well with public attitudes concerning believability. Given a chance to rate specific sources, the public makes clear that technology (print versus video news) is not the hook upon which its opinion hang. In essence, this debate about print versus broadcast news is somewhat misdirected." 22

Zumindest erwägenswert scheint zudem, daß das Vermittlungspersonal die Einschätzung beeinflußt: sowohl bestimmte, namentlich bekannte Journalisten als auch Nachrichtensprecher können hier eine Rolle spielen. So gibt es Hinweise, daß Fernsehen und Zeitungen mittels verschiedener Kriterien beurteilt werden: Beim Fernsehen ist die Glaubwürdigkeit in erster Linie abhängig von

Publishers Association, 4. November 1974), zitiert nach: Gantz (1981), S. 156.

20

Vgl. John D. Abel / Michael O. Wirth: Newspaper vs. TV Credibility for Local News, in: Journalism Quarterly 54/1977, S. 371-375; Michael Burgoon / Judee K. Burgoon / Miriam Wilkinson: Newspaper Image and Evaluation, in: Journalism Quarterly 58/1981, S. 411-419, 433; Raymond S. H. Lee: Credibility of Newspaper and TV News, in: Journalism Quarterly 55/1978, S. 282-287; Joey Reagan / Jayne Zenaty: Local News Credibility: Newspapers vs. TV Revisited, in: Journalism Quarterly 56/1979, S. 168-172. Speziell zur Einschätzung der Fairness lokaler Zeitungen siehe Charles T. Salmon / Jung-Sook Lee: Perceptions of Newspaper Fairness: A Structural Approach, in: Journalism Quarterly 60/1983, S. 663-670.

$21 \quad$ Vgl. Salmon / Lee (1983).

22 Michael J. Robinson / Andrew Kohut: Believability and the Press, in: Public Opinion Quarterly 52/1988, S. 174-189, S. 188. Glaubwürdigkeit wurde hier mit der folgenden Frage operationalisiert: "Please rate how much you think you can believe each organisation (the following people) I name on a scale of 4 to 1 . On this 4-point scale ' 4 ' means you can believe all or most of what they say and '1' means you can believe almost nothing of what they say." (S. 176) [Hervorhebung jeweils im Original.] 
Nachrichtenpräsentatoren, also von Individuen; die Zeitung wird dagegen eher als Institution wahrgenommen und beurteilt. Die Roper-Frage negiert diese Unterschiede und zwingt ihre Beantworter zu einer Entscheidung, ohne die zugrundeliegenden Kriterien zu berücksichtigen. ${ }^{23}$

Aber auch bei Printmedien kann die Person des Kommentators oder Leitartiklers die Glaubwürdigkeit einer Mitteilung erhöhen: In Sargents Untersuchung werden "personal news sources" durchweg als aufrichtiger, sorgfältiger, verantwortungsvoller und unvoreingenommener eingestuft als "impersonal sources" (Medien oder Sendungen in ihrer Gesamtheit), allerdings nur dann, wenn sie den Befragten namentlich bekannt sind. Allein die Hinzufügung eines (fiktiven) Namens führt nicht zu einer Steigerung der Glaubwürdigkeit. ${ }^{24}$

Durchweg hohe Werte für Präsentatoren, Korrespondenten und - mit etwas Abstand - auch Kommentatoren ermitteln auch Robinson/Kohut. Die namentlich bekannten "Anchormen" schneiden in allen Fällen besser ab als ihre Sendeanstalten: "There is little question ... that the public does award (or deny) believability on the basis of celebrity, or at least 'visibility'."25 Dieser Verantwortung ist sich auch Ulrich Wickert, Präsentator des Nachrichtenmagazins 'tagesthemen' (ARD), bewußt:

"... Glaubwürdigkeit zu erlangen ist ein schweres Unterfangen. Es reicht nicht, das Gewand entsprechend zu schneidern, sondern die Inhalte und diejenigen, die die Inhalte transportieren, wie auch diejenigen, die die Inhalte darbieten, müssen allesamt in diese Kategorie passen." 26

\footnotetext{
$23 \quad$ Vgl. Newhagen / Nass (1989), S. 278ff.

24 Vgl. Leslie W. Sargent: Communicator Image and News Reception, in: Journalism Quarterly 42/1965, S. 35-42, S. 38. Für das Fernsehen tritt dieser Effekt verstärkt auf. Der Befund für Printmedien wird gestützt durch Bradley S. Greenberg / Percy H. Tannenbaum: The Effects of Bylines on Attitude Change, in: Journalism Quarterly 38/1961, S. 535-537: "High Prestige Sources" (z.B. Walter Lippmann), die zu Beginn eines Zeitungsartikels durch Verfasserangabe kenntlich gemacht werden, verursachen eine verstärkte Meinungsänderung. 
Allerdings merkt er einschränkend an:

"... Glaubwürdigkeit kann nicht ausschließlich eine Moderatorin oder ein Sprecher vermitteln. Die Auswahl der Nachrichten, die Sprache, in der sie formuliert sind, die Qualität der Korrespondentenberichte etc. gehören alle zusammen. Wenn aber ein mit hoher Glaubwürdigkeit versehener Moderator von einer anerkannt seriösen Nachrichtensendung in eine Nachrichtensendung wechselt, der es in den Augen der Zuschauer an Ernsthaftigkeit und Objektivität fehlt, verliert auch derjenige an Gewicht, der das Äußere darstellt." ${ }^{27}$

Carter/Greenberg bemängeln zusätzlich, daß die Roper-Frage in zweifacher Hinsicht zu Verzerrungen führt: Zum einen gehen sie davon aus, daß sowohl Fernsehen als auch Tageszeitung durch den Vergleich mit Zeitschriften im Nachteil sind, da letzteren zwischen Ereignis und Berichterstattung mehr Zeit zur Verfügung steht. Dahinter steht wohl die Auffassung, daß die längere Zeitspanne den Zeitschriften die Möglichkeit zu vertiefender Recherche und Aufarbeitung gibt. Diese Überlegung scheint allerdings bei der Einschätzung durch die Rezipienten keine Rolle zu spielen; vielmehr wird Aktualität, wie

Ebd. S. 122f. Daß die Glaubwürdigkeit eines Moderators nicht nur dann leiden kann, wenn er zu einem Konkurrenzsender wechselt, mußte WICKERT 1995 am eigenen Leib erfahren: Als bekannt wurde, daß er in einem Werbefilm für die Versicherungsgesellschaft Deutscher Herold auftrat, wurde diese Nebentätigkeit scharf kritisiert; ein Rundfunkratsmitglied sah die Glaubwürdigkeit der 'tagesthemen' durch den Auftritt des ARD-Stars gefährdet. (Vgl. o.V.: "Ich bedauere den Vorfall", in: Süddeutsche Zeitung vom 14.11.1995. Siehe auch: Cornelia Bolesch: Versicherungsfritze Wickert? Nicht dumm, ehrlich! In: Süddeutsche Zeitung vom 14.11.1995; U. St.: Tugend-TV, in: Die Zeit vom 17.11.1995 sowie Volker Lilienthal: Wickerts Seitensprung, in: Journalist 12/1995, S. 43. Zur Glaubwürdigkeit von Journalisten, die Produktwerbung machen siehe auch: Volker Lilienthal: Riskantes Tingeln, in: Journalist 7/1995, S. 32-35.

In die Diskussion geriet 1995 auch kurzzeitig das Geschlecht der Präsentatoren. Unter Berufung auf Umfragen in England und USA erklärte der Moderator des 'heute-journals' (ZDF), Alexander NIEMETZ, daß Frauen vom Publikum als Moderatorinnen von News-Sendungen nicht akzeptiert würden. Glaubwürdig seien vielmehr "leicht ergraute und erfahrene Herren". Nicht ohne Süffisanz verwiesen daraufhin verschiedene Medien auf die zufällige Übereinstimmung dieser Beschreibung mit dem Aussehen des Herrn Niemetz. (Vgl. Bernadette Calonego: "So wird's gemacht, Frau Caniga!" Wie ein Schweizer Boulevardblatt gegen eine neue Fernseh-Chefredakteurin agiert, in: Süddeutsche Zeitung vom 19.10.1995; Heidrun Noblé: Grau in grau, Herr Niemetz! In: Die Woche vom 27.10.1995; o.V.: Brehmer über TV-Frauen, in: Süddeutsche Zeitung vom 21./22.10.1995.) 
oben erwähnt, ja gerade als ein Indikator für Glaubwürdigkeit betrachtet. Zum anderen benachteiligt ihrer Überzeugung nach die Art der Fragestellung die Tageszeitung, da insbesondere im Fall widersprüchlicher Berichte das Fernsehen wegen der visuellen Dimension im Vorteil sei. ${ }^{28}$ Sie plädieren daher dafür, die relative Glaubwürdigkeit für jedes Medium getrennt zu erheben. Ihre Frage lautet entsprechend:

"We would like your opinion on the reliability of (e.g. radio) for news. If perfect reliability is 100 percent, in your opinion, what percentage of the news on (e.g. radio) do you believe (from 0 - 100\%)?"29

Diese Fragestellung erlaubt Rückschlüsse sowohl auf die generelle Glaubwürdigkeit von einzelnen Medien im 'Normalfall' als auch auf die Frage, für wie glaubwürdig Medien insgesamt generell gehalten werden. ${ }^{30}$

Ein weiterer Einwand zielt nicht auf die Fragestellung, sondern auf die Interpretation der Roper-Befunde. Bemängelt wird, daß das Vorhandensein von Mehrfachantworten nicht berücksichtigt wird: "It is correct to infer from the Roper data that $59 \%$ believe they rely on television as one of their major sources of news but incorrect to make the common inference that television ist the major source for 59 \%."

\subsection{Mehrdimensionale Ansätze}

$28 \quad$ Carter / Greenberg (1965), S. 30

29

Ebd. S. 31 [Hervorhebung im Original]. Auch mit dieser Fragestellung ergibt sich noch ein deutlicher Vorsprung des Fernsehens und auch des Hörfunks gegenüber der Zeitung. Die durchschnittlichen Werte für die Zuverlässigkeit lauten: Fernsehen $82 \%$, Hörfunk $77 \%$, Zeitung $68 \%$. Zeitschriften wurden in den Vergleich nicht aufgenommen.

30

Vgl. Gantz (1981), S. 156.

31 Vernon A. Stone: Sources of Most News: Evidence and Inference, in: Journal of Broadcasting 14/1969-70, S. 1-4, S. 2. [Hervorhebung im Original.] 
Schließlich wird beanstandet, daß die Glaubwürdigkeit in bezug auf Medien zu allgemein betrachtet wird:

"These data are interesting, but they do not tell us much. Presumably, credibility as a media attribute has several components, not unlike the factors that have been isolated for source credibility or ethos. The significance of the Roper findings would be enhanced if the dimensions that form the basis for public acceptance of television as 'most believable' could be specified." ${ }^{32}$

Eine der Hauptstrategien, diese Dimensionen der Glaubwürdigkeit zu ergründen, ist die Verwendung von Semantischen Differentialen, die einer Faktorenanalyse unterzogen werden. ${ }^{33}$ Diese methodische Verlagerung geht nicht selten einher mit einer Ausweitung der Fragestellung auf das MedienImage insgesamt. ${ }^{34}$ Die Dimensionen, die sich dabei ergeben, stimmen zum größten Teil überein mit denen, die auch für die Quellenglaubwürdigkeit ermittelt wurden; Unterschiede liegen weniger in den inhaltlichen Merkmalen als in der Benennung der Dimensionen. McCroskey/Jenson etwa finden, neben dem Faktor 'Kompetenz,' noch 'Character' (der zumindest teilweise mit 'Vertrauenswürdigkeit' übereinstimmt), 'Sociability' (der auf Umgänglichkeit und Freundlichkeit verweist), 'Composure' (Hinweise auf die Gemütsverfassung wie 'entspannt' oder 'nervös') sowie 'Extroversion' (der Übereinstimmungen mit dem bekannten Faktor 'Dynamik' aufweist). ${ }^{35}$

32 Shaw (1973), S. 306. Eine ähnliche Kritik findet sich bei Burgoon / Burgoon / Wilkinson (1981), S. 412.

33 Vgl. z.B. die Studien von Jacobson (1969), Shaw (1973) und Lee (1978).

34 Vgl. z.B.: James C. McCroskey / Thomas A. Jenson: Images of Mass Media News Sources, in: Journal of Broadcasting 19/1975, S. 169-180. Ebenso wie im Bereich der QuellenGlaubwürdigkeit ist auch hier die Gleichsetzung von 'Glaubwürdigkeit' und 'Image' abzulehnen. Zwar ist Bentele zuzustimmen, wenn er konstatiert, daß Glaubwürdigkeit ein wichtiger Imagefaktor der Medien ist (vgl. Günter Bentele: Objektivitätsanspruch und Glaubwürdigkeit, in: Otfried Jarren (Hrsg.): Medien und Journalismus 1. Eine Einführung, Opladen 1994, S. 295312, S. 296), sie ist aber eben nur ein Faktor. Zu behaupten, daß alle wahrgenommenen Anmutungen zur Glaubwürdigkeit beitragen, ist für die Klärung des Konzepts nicht hilfreich. 
Lee benennt neben 'Trustworthiness', 'Expertness' und 'Dynamism' noch 'Objectivity' und 'Bias' (die nicht klar zu trennen sind, jedenfalls aber auf Unparteilichkeit bzw. Voreingenommenheit verweisen) sowie 'Agreeableness' (hier kommt allgemeine Sympathie sowie Ähnlichkeit mit dem Rezipienten zum Ausdruck), 'Intimacy' (verweist auf Nähe, 'Involvement' und Aspekte der Gestaltung wie z.B. 'visual' und 'colorful') und 'Availability' (die Frage der Möglichkeit des Zugangs zum Medium). ${ }^{36}$ Jacobson unterscheidet vier Dimensionen, von denen er zwei als Glaubwürdigkeits-Dimensionen bezeichnet ('Authenticity' als Mischung von Kompetenz- und Vertrauenswürdigkeitsbeschreibungen und 'Objectivity') und zwei - ohne nähere Begründung - als Unglaubwürdigkeits-Dimensionen ('Dynamism' und 'Respite' mit den Ausprägungen 'relaxed-tense' und 'pleasant-unpleasant'). ${ }^{37}$

Zwar basieren die faktorenanalytischen Studien, die mit Semantischen Differentialen arbeiten, auf einem breiteren Glaubwürdigkeitskonzept als die eindimensionale Operationalisierung mittels der Roper-Frage, ein kritischer Einwand sollte allerdings nicht unberücksichtigt bleiben:

"Die Mehrzahl dieser Arbeiten [bezieht] die Glaubwürdigkeitszuschreibung der Medien auf Variablen, die sich auf Eigenschaften von 'Akteuren' beziehen. Folglich enthalten sie Dimensionen, die lediglich personenbezogene Beurteilungsmaßstäbe wiedergeben. Diese werden den Rezipienten dann aber zur Beurteilung von Institutionen (oft intermediale Vergleiche zwischen Fernsehen, Presse, Radio) vorgelegt. Das führt dazu, daß nicht mehr zu unterscheiden ist, ob aufgrund der Adjektivvorgabe eine Personenbeurteilung oder eine Institutionsbeurteilung stattgefunden hat. Angemessener sind vermutlich Konzepte, die auf der Variablen- und der Stimulusebene sowohl die Personen- als auch die Institutionskomponente der Medien berücksichtigen, denn es ist davon auszugehen, daß Rezipienten zwischen Personen und Institutionen differenzieren." ${ }^{38}$

36 Vgl. Lee (1978).

37 Harvey K. Jacobson: Mass Media Believability: A Study of Receiver Judgments, in: Journalism Quarterly 46/1969, S. 20-28, S. 22f.

38

Susanne Deimling / Jürgen Bortz / Gerhard Gmel: Zur Glaubwürdigkeit von Fernsehanstalten. Entwicklung und Erprobung eines Erhebungsinstrumentes, in: Medienpsychologie 5/1993, S. 203-219, S. 206. Insbesondere bei der Bewertung von Fernsehanstalten oder programmen könnte sich die Unterscheidung von Personen und Institution als bedeutsam 
$\mathrm{Zu}$ berücksichtigen ist ferner der bereits präsentierte Einwand ${ }^{39}$, daß die Beschaffenheit der Faktoren abhängig ist vom "Input", also von den zugrundeliegenden Semantischen Differentialen: Faktoren können sich nur dann ergeben, wenn die entsprechenden Adjektive vorhanden sind. Da die Instrumente meist in Anlehnung an vorangegangene Studien konzipiert sind, und kaum hinterfragt wird, ob die verwendeten Adjektive von Rezipienten tatsächlich für Glaubwürdigkeitsbeurteilungen herangezogen werden bzw. ob diese die einzigen Elemente sind, die herangezogen werden, ist mit eingeschränkten Ergebnissen zu rechnen.

Den Nachweis, daß mehr Faktoren als herkömmlich postuliert in Zusammenhang mit Glaubwürdigkeit stehen, führt Singletary auf folgende Weise ${ }^{40}$ : Er läßt zunächst 90 Studenten in freier Assoziation niederschreiben, was eine Nachrichtenquelle ihrer Wahl (eine reale Person aus Fernsehen, Hörfunk, Zeitung, Zeitschrift, Nachrichtenagentur oder Buch) in ihren Augen glaubwürdig macht. Das Resultat - eine Liste mit 203 Wörtern - wurde dann einer anderen Gruppe von Studenten vorgelegt, die auf einer sechsstufigen Skala angeben sollten, wie eng der Zusammenhang jedes Wortes mit Glaubwürdigkeit ihrer Einschätzung nach ist.

Die anschließende Faktorenanalyse erbrachte insgesamt 41 Faktoren, wobei die ersten sechs Faktoren bereits 48 Prozent der Varianz erklären, während die übrigen Faktorladungen äußerst gering waren. Zu den Hauptfaktoren gehören neben 'Kompetenz' (hier: "Knowledgeability") und 'Vertrauenswürdigkeit' noch "Attraction", "Articulation", "Hostility" und "Stability". "Attraction" umfaßt Sympathiemerkmale wie humorvoll, freundlich und warm. "Articulation" beinhaltet Merkmale der Ausdrucksweise wie Klarheit, Schlüssigkeit und Verständlichkeit, die bisher im Zusammenhang mit Medien nicht diskutiert wurden und wohl deshalb Bedeutung erlangten, weil hier ausdrücklich Personen zur Beurteilung standen. "Hostility" ist gekennzeichnet durch die

erweisen. Siehe auch: Robinson / Kohut (1988). 
Adjektive herausfordernd, zynisch und satirisch. Dieser auf den ersten Blick verblüffende Befund wird dahingehend interpretiert, daß sich die "Feindseligkeit" der Quelle in der Wahrnehmung der Versuchspersonen wohl nicht gegen das Publikum richte, sondern gegen Dritte, etwa Politiker, ${ }^{41}$ mithin also als Ausdruck der Kritikfunktion der Publizisten gewertet werden kann. "Stability" schließlich wird repräsentiert durch Kennzeichnungen wie gemäßigt (non-radical), nicht emotional und nicht polemisch. Der Faktor weist damit inhaltliche Nähe zu Aspekten der Vertrauenswürdigkeit auf (Neutralität, Fairneß).

Neben den bekannten Faktoren ergeben sich also einige neue; zusätzlich umfassen die genannten sechs Faktoren noch eine Reihe von "sub-clusters", die Singletary zu der Schlußfolgerung veranlassen: "Credibility was found not to be a simple, well differentiated system of factors, but a highly complex and somewhat undifferentiated system of factors. Where many variables were given the opportunity to appear in the data, many did appear." ${ }^{42}$ Diese Einschätzung ist allerdings zu relativieren: Zwar zeigt diese rezipientenorientierte Vorgehensweise eine Vielzahl neuer Aspekte auf, die nicht außer Acht gelassen werden sollten, diese sind jedoch - wie die schwachen Faktorladungen belegen - stark an individuelle Vorstellungen gebunden. Zugleich ergibt sich eine Bestätigung der Relevanz derjenigen Faktoren, die auch bisher schon als bedeutsam eingestuft wurden.

\subsection{Spezifische Merkmale der Glaubwürdigkeit von Medien}

In der letztgenannten Studie von Singletary deutete sich an, daß für die Beantwortung der Frage, was die Glaubwürdigkeit von Medien ausmacht, die Verwendung speziell medienbezogener Kriterien hilfreicher ist, als die Vorlage allgemein gehaltener Semantischer Differentiale. Einen solchen alternativen Weg zur Ermittlung der Glaubwürdigkeit wählen auch Gaziano/McGrath: In

\footnotetext{
$41 \quad$ Vgl. Singletary (1976), S. 319.

$42 \quad$ Singletary (1976), S. $318 f$.
} 
Gruppendiskussionen ermitteln sie eine Reihe von Kriterien, die sie dann mittels schriftlicher und telefonischer Befragung einschätzen lassen. Die nachfolgende Faktorenanalyse ergibt für den Faktor 'Glaubwürdigkeit' folgende relevante Items:

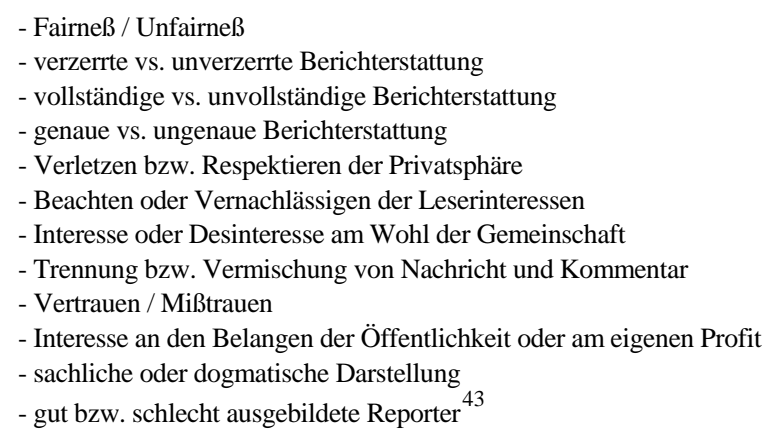

Der Befund bestätigt die zentralen Dimensionen 'Kompetenz' und 'Vertrauenswürdigkeit' und liefert gleichzeitig konkrete Hinweise darauf, welche Elemente aus Sicht der Rezipienten zu diesen Dimensionen gehören. Eine Präzisierung des Instruments unternahm Meyer, der zugleich fand, daß der Aspekt der 'Sozialen Billigung' auch für Medien relevant werden kann:

"A newspaper can be believed but still be alienated if it advocates positions strongly opposed by a majority in its community or undertakes investigations or editorial positions that run counter to the perceived economic or social interests of the community." 44

43 Vgl. Gaziano / McGrath (1986), S. 454f. Daneben benennen sie noch einen Faktor 'Social Concern', der die folgenden Ausprägungen umfaßt:

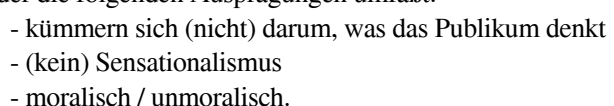

Philip Meyer: Defining and Measuring Credibility Of Newspapers: Developing an Index, in: Journalism Quarterly 65/1988, S. 567-574, 588, S. 567. Zur Auseinandersetzung mit den Skalen von Gaziano/McGrath und Meyer vgl. auch Mark Douglas West: Validating a Scale for the Measurement of Credibility: A Covariance Structure Modeling Approach, in: Journalism Quarterly 71/1994, S. 159-168. 
Konkrete, speziell medienbezogene Items zur Operationalisierung der Glaubwürdigkeit von Medien finden sich auch bei Iyengar/Kinder, die Objektivität sowie Exaktheit und Sorgfalt (accuracy) einschätzen lassen. ${ }^{45} \mathrm{Wanta} / \mathrm{Hu}$ veranschaulichen diese Merkmale und machen die Glaubwürdigkeit von Medien abhängig vom Grad der Ablehnung der folgenden Statements: "'News organizations such as newspapers and television news try to manipulate public opinion', 'News organizations often fail to get all of the facts straight', 'News organisations often don't deal fairly with all sides of a political or social issue', and 'News organizations do a poor job of separating facts from opinions"'. ${ }^{46}$

Sachgerechter Umgang mit Fakten sowie Genauigkeit und Sorgfalt im Umgang mit dem Nachrichtenmaterial betreffen die Beherrschung der journalistischen Handwerksregeln und sind somit primär Indizien für die Kompetenz. Allerdings können in der Einschätzung der Leser bei einem Verstoß gegen die Sorgfaltspflicht auch Abstriche hinsichtlich der zugeschriebenen Vertrauenswürdigkeit wahrgenommen werden. Eine ungenaue Wiedergabe von Zitaten etwa wird als Indiz für die Parteilichkeit von Journalisten betrachtet. ${ }^{47}$

45 Vgl. Shanto Iyengar / Donald R. Kinder: Psychological Accounts of Agenda-Setting, in: Sidney Kraus / Richard M. Perloff (Hg.): Mass Media and Political Thought. An InformationProcessing Approach, Beverly Hills / London / New Delhi 1985, S. 117-140, S. 124.

46

Wayne Wanta / Yu-Wei Hu: The Effects of Credibility, Reliance, and Exposure on Media Agenda-Setting: A Path Analysis Model, in: Journalism Quarterly 71/1994, S. 90-98, S. 94.

47

Vgl. Douglas J. Kocher / Eugene F. Shaw: Newspaper Inaccuracies and Reader Perceptions of Bias, in: Journalism Quarterly 58/1981, S. 471-474, 516. Siehe auch C. Edward Wilson / Douglas M. Howard: Public Perception of Media Accuracy, in: 55/1978, S. 73-76, S. 75. Fehler in der Berichterstattung werden hier von den Befragten sowohl auf Inkompetenz wie auch auf Einseitigkeit zurückgeführt. Zum Einfluß von Rechtschreib-, Grammatik- und Interpunktionsfehlern auf die Glaubwürdigkeit siehe Bradley S. Greenberg / Edward L. Razinsky: Some Effects of Variations in Message Quality, in: Journalism Quarterly 43/1966, S. 486-492; zum Einfluß von Fehlern im Wortgebrauch und im logischen Aufbau siehe Michael W. Singletary / Kevin Carragee: Subjective Errors in Written Messages, in: Journalism Quarterly 57/1980, S. 432-437. Zu den Forschungsbemühungen bezüglich fehlerhafter oder ungenauer Berichterstattung allgemein siehe Mitchell V. Charnley: Preliminary Notes on A Study of Newspaper Accuracy, in: Public Opinion Quarterly 13/1936, S. 394-401; Fred C. 
Unabhängig vom gewählten methodischen Zugang zeichnet sich als Defizit ab, daß in den meisten Fällen nur das Medium als Ganzes im Zentrum der Fragestellung steht; es wird also weder zwischen einzelnen Ressorts noch zwischen einzelnen Sendungen, Sendungstypen oder Darstellungsformen unterschieden. Eine durchgehend differenziertere Betrachtungsweise steht noch aus.

\subsection{Zusätzliche Einflußfaktoren}

\subsubsection{Abhängigkeit vom Rezipienten}

Dagegen wurde die Forderung, auf seiten der Rezipienten genauer zu untersuchen, wer welches Medium für glaubwürdiger hält, häufiger aufgegriffen. Die Rezipienten werden dabei in der Regel nach soziodemographischen Variablen unterschieden. ${ }^{48}$ Die Befunde hinsichtlich Geschlecht, Bildung und Alter sind nicht durchweg konsistent: Stellen ältere Studien noch fest, daß Frauen eher das Fernsehen und Männer eher die Zeitung für glaubwürdiger halten $^{49}$, wird die entsprechende Hypothese in einer jüngeren Untersuchung zurückgewiesen. $^{50}$ Ähnliche Uneinigkeit gibt es hinsichtlich der Variable

Berry: A Study of Accuracy in Local News Stories of Three Dailies, in: Journalism Quarterly 44/1967, S. 482-490; Gerald L. Grotta: Attitudes on Newspaper Accuracy and External Controls, in: Journalism Quarterly 46/1969, S. 757-759; Gary C. Lawrence / David L. Grey: Subjective Inaccuracies in Local News Reporting, in: Journalism Quarterly 46/1969, S. 753-

757; T. Joseph Scanlon: A New Approach to Study of Newspaper Accuracy, in: Journalism Quarterly 49/1972, S. 587-590. Die Benennung der Mängel in einzelnen Beiträgen erfolgt in der Regel durch die Primärquellen, die in diesen Beiträgen zu Wort kommen. Eine solche Befragung von Primärquellen findet sich auch bei: Detlef Schröter: Qualität und Journalismus. Theoretische und praktische Grundlagen journalistischen Handelns, München 1995. Gefragt wurde hier unter anderem, ob die Angaben zur Person korrekt waren und ob die Aussagen verfälscht wurden.

Vgl. Shaw (1973), S. 308

49

Vgl. Westley / Severin (1964), S. 330 und Greenberg (1966), S. 667.

50

Vgl. Mulder (1980), S. 476. 
'Alter': Jüngere halten die Zeitung für etwas glaubwürdiger als Ältere, wenngleich in allen Altersgruppen die Glaubwürdigkeit des Fernsehens überwiegt ${ }^{51}$; Ältere halten die Zeitung für glaubwürdiger ${ }^{52}$; Alter ist kein relevanter Indikator $^{53}$; so lauten die widersprüchlichen Ergebnisse. Auch daß besser Gebildete die Zeitung bevorzugen und weniger Gebildete eher das Fernsehen ${ }^{54}$, findet nicht generell Bestätigung. ${ }^{55}$ Vielmehr wird meist beobachtet, daß trotz geringer Unterschiede Fernsehen über alle Bildungsgruppen hinweg als am glaubwürdigsten erscheint. ${ }^{56}$ Diese Uneinigkeit in den Befunden ist unter Umständen, ebenso wie die Differenz zwischen Fernsehen und Zeitung, ein Produkt der Fragestellung:

"When asked directly about the believability of news organizations and newspeople - specific news organizations and people - Americans express generally positive opinions about the press. Nor is there very much variation in opinion about press believability among the various political and sociological groups that typically divide public opinion in America. To suppose that believability of the press varies substantially from one demographic or ideological group to the next is, to a surprising degree, an incorrect inference." ${ }^{57}$

Gunther lehnt die Betrachtung der soziodemographischen Merkmale der Rezipienten im Zusammenhang mit der Glaubwürdigkeit der Medien als "atheore-

$51 \quad$ Vgl. Westley / Severin (1964), S. 330.

$52 \quad$ Vgl. Mulder (1980), S. 476.

53 Vgl. Greenberg (1966), S. 668. In einer anderen Befragung wurde ermittelt, daß insbesondere Jugendliche Medien als nicht vertrauenswürdig einstufen. Sie glauben, daß Medien einer Zensur unterliegen und die Nachrichten zugunsten der Regierung einfärben. Diese Skepsis betraf vor allem Meldungen über Vietnam, daher sind die Befunde nicht verallgemeinerbar. Vgl. Bishop / Boersma / Williams (1969).

Vgl. Westley / Severin (1964), S. 327 und Greenberg (1966), S. 668.

56 Vgl. z.B. Carter / Greenberg (1965), S. 33 und Reagan / Zenaty (1979), S. 170.

57 Robinson / Kohut (1988), S. 175. 
tisch" ab und spricht sich dafür aus, genauer zu untersuchen, unter welchen Bedingungen die Berichterstattung als glaubwürdig empfunden wird:

"Credibility is not a trait that people ascribe consistently to a channel, but, rather a highly situational assessment. (...) The situational approach proposes that important components of the trusting or sceptical response do not exist until a person has something to be trusting or sceptical about." $^{58}$

Einstellungen und Überzeugungen der Rezipienten werden - anders als bei personalen Quellen - im Zusammenhang mit Medien als Quellen in der Regel nicht hinterfragt, obwohl durchaus davon ausgegangen werden kann, daß unterschiedliche Glaubwürdigkeitseinschätzungen vorgenommen werden, je nachdem, ob etwa die politischen Einstellungen eines Rezipienten mit der (vermuteten oder tatsächlichen) politischen Ausrichtung eines Mediums konform gehen oder nicht. Lediglich in einer deutschen Untersuchung wird dieser Frage am Rande nachgegangen. Die Verfasser resümieren:

"Prüfbar waren ... die Hypothesen, daß zunehmende Attraktivität und zunehmender Sachverstand eines Kommunikators [gemeint ist hier: einer Zeitung] die Attitüden der Rezipienten stärker in Richtung des Kommunikators verändern. Es konnte gezeigt werden, daß diese Beziehung deutlicher nachweisbar ist, wenn die Glaubwürdigkeit des Kommunikators insgesamt niedriger ist. Offenbar wird die Frage, ob der Kommunikator mehr oder weniger sympathisch bzw. sachverständig ist, erst relevant, wenn es bei niedriger Glaubwürdigkeit aufgrund genügend großer Attitüdendiskrepanzen überhaupt zu Attitüdenänderungen kommt. Argumentiert demgegenüber ein Kommunikator in ähnlicher Weise wie der Rezipient, ist seine Glaubwürdigkeit weniger wichtig."

\subsubsection{Abhängigkeit vom Thema}

$58 \quad$ Albert C. Gunther: Biased Press or Biased Public? Attitudes toward Media Coverage of Social Groups, in: Public Opinion Quarterly 56/1992, S. 147-167, S. 149. Siehe auch die Ausführungen ebd. S. 147ff.

Jürgen Bortz / Konrad Leitner: Zur Frage der Beziehung zwischen der attitüdenändernden Wirkung zweier Tageszeitungen und ihrer Bewertung, in: Zeitschrift für Sozialpsychologie 10/1979, S. 70-84, S. 83. 
Mit der letztgenannten Betrachtung ist angedeutet, daß die Glaubwürdigkeit der Medien auch abhängig ist vom Gegenstand der Berichterstattung. Einzelne Untersuchungen prüfen entsprechend den Zusammenhang von Glaubwürdigkeit und Thema. Gantz experimentiert mit einer groben Kategorisierung in 'Nachrichten', 'Sport' und 'Wetter' und kommt dabei zu dem wenig überraschenden Ergebnis, daß Sportmeldungen am häufigsten (86.8\%) und Wettermeldungen am seltensten (71.1\%) geglaubt werden. ${ }^{60}$ Das relative Mißtrauen dem Wetterbericht gegenüber resultiert wohl aus der unmittelbar gegebenen Nachprüfbarkeit für die Rezipienten und dem damit verbundenen Umstand, daß wohl jeder schon einmal Erfahrungen mit falschen Vorhersagen gemacht hat. Im Falle von Sportmeldungen handelt es sich dagegen meist nicht um Prognosen; zumindest im Bereich der Ergebnisberichterstattung ist hier eine hohe Verläßlichkeit durch relative Nachprüfbarkeit gegeben. Wie Mukenhaupt ironisch anmerkt, erhöht sich die Glaubwürdigkeit der Sportberichterstattung in den letzten Jahren in besonderem Maße dadurch, daß die gleichen Ergebnisse auf immer mehr Kanälen präsentiert werden. ${ }^{6}$

Ryan untersucht, ob es Zusammenhänge gibt zwischen verschiedenen Themen von Nachrichten, ihrem Lokalbezug und ihrer Glaubwürdigkeit. Seine Analyse ergibt, daß das Fernsehen glaubwürdiger bei der Vermittlung des Themas Studentendemonstrationen ist, egal ob diese auf regionaler, nationaler oder internationaler Ebene stattfinden (wahrscheinlich wegen der Visualisierung). Für öffentliche Angelegenheiten des Bundesstaates und für Wissenschaft scheint die Zeitung glaubwürdiger zu sein als das Fernsehen (möglicherweise, weil diese Themen im Fernsehen nicht oder selten behandelt werden). ${ }^{62}$

Im Fall 'Watergate' wurden Presse und Fernsehen als gleichermaßen wichtige Informationsquellen eingestuft. Glaubwürdigkeit war nicht das

$60 \quad$ Vgl. Gantz (1981), S. 160.

61 Vgl. Manfred Muckenhaupt: Die neue Qualität des Mediensports. Auswirkungen der Medienkonkurrenz auf die Sportberichterstattung des Fernsehens, in: Brennpunkte der Sportwissenschaft 4/1990, S. 5-19, S. 7. 
wichtigste Kriterium für die Wahl des Mediums, wurde aber von knapp einem Viertel der Befragten als Grund angeführt. ${ }^{63}$

Gemeinsam ist diesen Untersuchungen, daß sie sehr allgemein gehalten sind. Erst in jüngerer Zeit wird berücksichtigt, daß individuelle Themenpräferenzen oder Einstellungen auf Rezipientenseite in Zusammenhang mit der Glaubwürdigkeit stehen können. Gunther zeigt, daß das Ausmaß der persönlichen Betroffenheit ('issue involvement'), das sich, wie gezeigt, ${ }^{64}$ auf die Glaubwürdigkeit der Quellen auswirkt, auch die Glaubwürdigkeit der Medienberichterstattung über ein bestimmtes Thema beeinflußt. Das Vertrauen in die Berichterstattung war dann am größten, wenn bezüglich des Themas gemäßigte Einstellungen bei den Rezipienten zu verzeichnen waren; bei extrem polarisierten oder neutralen Einstellungen sank das Vertrauen. ${ }^{65}$ Daß starkes 'Involvement' zu mehr Skepsis den Medien gegenüber führt, wird auch an anderer Stelle ausgeführt. ${ }^{66}$

Die Beziehung des Rezipienten zum Inhalt der Mitteilung wird von Stamm/Dube ebenfalls genauer betrachtet. Ihre Untersuchung basiert auf einem multidimensionalen Einstellungskonzept, das neben dem 'Involvement' des Rezipienten die Richtung, Stärke und Stabilität seiner Einstellung umfaßt. Diese Komponenten werden für insgesamt sechs politische Themen (Handelsbeziehungen mit Japan, Todesstrafe, Krankenversicherung, Drogenkrieg, Subventionierung von Ausbildung und Schutz von Feuchtgebieten) ermittelt; zusätzlich wird gefragt, in welchem Ausmaß (auf einer 10-Punkt-Skala) der Berichterstattung zum jeweiligen Thema (in Fernsehen und Zeitung) vertraut wird. Bei fünf der sechs vorgegebenen Themen (Ausnahme war das Thema

\footnotetext{
63 Vgl. Edelstein / Tefft (1974), S. 433.

$64 \quad$ Vgl. Kap. 4.6.3, S. $75 \mathrm{ff}$.

65 Vgl. Albert Gunther: Attitude Extremity and Trust in Media, in: Journalism Quarterly 65/1988, S. 279-287, S. 283

66

Vgl. Gunther (1992), S. 150 sowie Mark P. Zanna / Ellen C. Klosson / John M. Darley: How Television News Viewers Deal with Facts that Contradict their Beliefs: A Consistency and Attribution Analysis, in: Journal of Applied Social Psychology 6/1976, S. 159-176 und Kitty Klein / Debra Holt: The Relationship of Need for Cognition and Media Credibility to Attitudes Toward the Persian Gulf War, in: Contemporary Social Psychology 15/1991, S. 166-171.
} 
'Handelsbeziehungen') ergab sich eine positive lineare Beziehung zwischen der Richtung der Einstellung und dem Zutrauen zur Berichterstattung. Hingegen konnten nur schwache Zusammenhänge der übrigen drei Komponenten mit der Glaubwürdigkeit der Medien nachgewiesen werden. ${ }^{67}$ Nicht zuletzt mag das daran liegen, daß "die Medien" nicht ähnlich differenziert wie die Rezipienten und ihre Einstellungen beleuchtet wurden. Da es "die Berichterstattung" zu einem Thema so pauschal nicht gibt, wäre zumindest zu prüfen, welche Medien (besser noch: welche Programme oder Sendungen) in der Einschätzung der Rezipienten welche Haltungen zum Thema einnehmen, um dann im Vergleich mit den Auffassungen der Rezipienten Rückschlüsse auf Glaubwürdigkeitszuschreibungen zu erlangen. Durchgeführt wurde eine solche Prüfung von Zanna/del Veccio, die die Hypothese bestätigen konnten, daß Zuschauer Fernsehnachrichten als glaubwürdiger wahrnehmen, wenn diese einen Standpunkt präsentieren, der ihrem eigenen ähnlich ist. ${ }^{68}$

Ein bislang unberücksichtigter Sonderfall von persönlicher Betroffenheit ist gegeben, wenn der Gegenstand der Berichterstattung die eigene Bezugsgruppe des Rezipienten ist. Gunther weist nach, daß die Glaubwürdigkeit der Medien von dieser Orientierung berührt wird:

"The hypothesis that group involvement is an important predictor of credibility judgments received good support in these data. In every case, and for both newspapers and television news, social group membership ... showed a strong relationship to judgments of news coverage. If a person identified with a particular group, he or she was significantly more likely to say the media gave unfavorable coverage to that group. In addition, group membership predicted credibility judgments more systematically than any of the other variables measured in this study." 69

67 Vgl. Keith Stamm / Ric Dube: The Relationship of Attitudinal Components to Trust in Media, in: Communication Research 21/1994, S. 105-123.

68

Vgl. Mark P. Zanna / Steven M. del Veccio: Perceived Credibility of Television News: A Matter of Viewer's Attitudes and the Position taken by the Media, in: European Journal of Social Psychology 3/1973, S. 213-216. Siehe auch Mark P. Zanna / Ellen C. Klosson / John M. Darley: How Television News Viewers Deal with Facts that Contradict their Beliefs: A Consistency and Attribution Analysis, in: Journal of Applied Social Psychology 6/1976, S. 159-176. 
Wenn die Berichterstattung über die 'Ingroup' als unfair empfunden wird, sinkt also die Glaubwürdigkeit der jeweiligen Medien. Die Empfehlung von Meyer: "chief and traditional cure for the credibility problem: writing and presenting the news without bias or favor toward or against any particular individual or group" $^{70}$ ist nur bedingt geeignet, hier Abhilfe zu schaffen, da selbst eine neutrale Berichterstattung bei den Betroffenen unter Umständen den Eindruck des Benachteiligtseins erzeugen kann.

\subsection{Befunde für die Bundesrepublik Deutschland}

Der Fülle an Studien im amerikanischen Raum steht eine eher geringe Anzahl von Untersuchungen in Deutschland gegenüber. Abgesehen von einer Reihe von Meinungsumfragen, die das amerikanische 'Office of Military Government' zwischen 1945 und 1949 initiierte, setzt die Forschung zur Glaubwürdigkeit von Medien erst 1962 ein. Eine EMNID-Umfrage, die sich an der RoperFragestellung orientierte, erbrachte damals 30 Prozent für den Hörfunk als glaubwürdigstes Medium, 23 Prozent für das Fernsehen und 17 Prozent für die Zeitung. ${ }^{71}$ In den folgenden Jahren (1964 und 1968) steigt, wie in den amerikanischen Untersuchungen, die Anzahl derjenigen deutlich, die bei einander widersprechenden Nachrichten am ehesten dem Fernsehen Glauben schenken, und zwar auch bei denjenigen Befragten, die sich bevorzugt aus Hörfunk oder Zeitung informieren. ${ }^{72}$ EMNID fragte zusätzlich nach einer Begründung dieser Entscheidung und erhielt für das Fernsehen vier Antwortgruppen: Die meisten Befragten ließen sich durch die visuelle Information

Philip Meyer: Elitism and Newspaper Believability, in: Journalism Quarterly 50/1973, S. 31-36, S. 31 .

$71 \quad$ Vgl. Bentele (1988), S. 413 und Bentele (1994), S. 299.

72 Vgl. Siegfried H. Drescher: Wirkung des Fernsehens als Quelle aktueller Informationen, in: Publizistik 14/1969, S. 173-184, S. 177f. Die Tatsache, daß 20 Prozent der Befragten auf diese Frage keine Antwort geben konnten oder wollten, kann dabei allerdings als Indiz für die Schwächen dieser Fragestellung gewertet werden. 
überzeugen ("die zeigen ja die Bilder und können deshalb nicht lügen"). Die zweite Gruppe leitete aus der Objektnähe der visuellen Information deren Objektivität ab ("die im Fernsehen können ja nur Tatsachen zeigen"). Eine weitere Gruppe sah im Fernsehen "so etwas wie eine amtliche, gut kontrollierte Institution..., der das Lügen verboten ist". Die vierte Gruppe schließlich benannte die Schnelligkeit der Information als wichtiges Kriterium und begründete das Vertrauen zum Fernsehen mit dessen größerer Aktualität. ${ }^{73}$

Bei der Frage, welcher Quelle bei widersprechenden Nachrichten am wenigsten Glauben zu schenken sei, gab ein Drittel der Befragten keine Stellungnahme ab, 47 Prozent nannten die Illustrierte, 17 Prozent die Zeitung, zwei Prozent das Fernsehen und ein Prozent den Hörfunk. Mißtrauen dem Fensehen gegenüber wurde begründet mit Einseitigkeit oder Abhängigkeit (vom Staat oder von Parteien) sowie mit Verfälschungen (z.B.: "wird alles nur ins schöne Licht gerückt", "weil das Fernsehen nur Demonstrationen und

73 Vgl. Drescher (1969), S. 178f. Der naive Glaube an die visuelle Information wurde im Januar 1996 nachhaltig erschüttert, als bekannt wurde, daß vor allem das Magazin 'Stern TV' (RTL) einem Fälscher aufgesessen war. Der Filmproduzent Michael Born verkaufte 'Stern-TV' elf Reportagen, die durchweg gefälscht und mit Laienschauspielern inszeniert waren. Diese gingen ohne Beanstandung durch die Redaktion auf Sendung. Als die Fälschungen im Zuge polizeilicher Ermittlungen offenbar wurden, entbrannte eine Diskussion um die Glaubwürdigkeit des Fernsehens. (Vgl. Oliver Kuhn: So belügt uns das Fernsehen, in: Abendzeitung von 19.01.1996; Oliver Kuhn / Michael Eder: Ein TV-Held am Ende? Jauch kämpft um seinen guten Ruf, in: Abendzeitung vom 30.01.1996; Michael Bitala: Als die Bilder lügen lernten, in: Süddeutsche Zeitung vom 10./ 11.02.1996; Hans Werner Kilz: Verirrter Journalismus, in: Süddeutsche Zeitung vom 10./11.02.1996; Burkhard Straßmann: Gut gefälscht ist halb gewonnen, in: Die Zeit vom 02.02.1996; Werner A. Perger: Unter Fälschern, in: Die Zeit vom 02.02.1996.) Lediglich in der 'Woche' wurde thematisiert, daß unter Umständen nicht nur Nachlässigkeiten der Redaktion bei der Recherche für den "Erfolg" des Fälschers verantwortlich waren: "Die Verantwortlichen stellen sich tot. Der Schuldige ist ausgemacht. Ein freier Journalist, der seine blühende Phantasie effektvoll ins Bild zu setzen wußte. Doch Vorsicht: So einfach kann der Fall nicht zu den Akten gelegt werden. Wie es aussieht ist der Mann auch angestiftet worden. Ihm wurde redaktionelle Hilfe zuteil, seine Dubiosität ist systematisch ignoriert worden." (Manfred Bissinger: Wenn Bilder lügen, in: Die Woche vom 16.02.1996.) 
Sensationen bringt"). ${ }^{74}$

Die adaptierte Roper-Frage nach der relativen Glaubwürdigkeit im Fall widersprüchlicher Meldungen wird von Berg/Kiefer seit 1970 gestellt. Seitdem wird Fernsehen als weitaus glaubwürdigstes Medium eingestuft, allerdings mit kontinuierlich abnehmender Tendenz (1970: 75 Prozent; 1990: 63 Prozent). Während der Hörfunk mit Werten zwischen 13 und 17 Prozent in der Einschätzung des Publikums weitgehend stabil bleibt, gewinnt die Zeitung an Glaubwürdigkeit (1970: 12 Prozent; 1990: 22 Prozent). ${ }^{7}$

Eine andere Studie operationalisiert Glaubwürdigkeit durch die Zustimmung zu der Aussage "berichtet wahrheitsgetreu". Anders als bei Berg und Kiefer werden Mehrfachnennungen zugelassen. Die Rangfolge lautet hier:

\begin{tabular}{|c|c|c|}
\hline - regionale Abo-Zeitungen: & & $63 \%$ \\
\hline - Fernsehen: & & $56 \%$ \\
\hline $\begin{array}{l}\text { - öffentl.-rechtl. Hörfunk: } \\
\text { - privater Hörfunk: }\end{array}$ & $21 \%$ & $38 \%$ \\
\hline - Anzeigenblätter: & & $18 \%$ \\
\hline - Zeitschriften/Illustrierte: & & $16 \%$. \\
\hline
\end{tabular}

Die Frage nach der wahrheitsgetreuen Berichterstattung wird von Berg und Kiefer ebenfalls gestellt, allerdings unter dem übergeordneten Gesichtspunkt

$74 \quad$ Vgl. Drescher (1969), S. 179.

75 Vgl. Klaus Berg / Marie-Luise Kiefer (Hrsg.): Massenkommunikation IV. Eine Langzeitstudie zur Mediennutzung und Medienbewertung 1964-1990, Baden-Baden 1992, S. 253ff.

76

Vgl. Regionalpresse e.V.: Einkaufs- und Informationsverhalten 1990. Die Medialeistung von Presse, Hörfunk und Fernsehen im Vergleich, Frankfurt a.M. 1991, S. 19f. Durchgeführt wurde die Befragung von der GfK Medienforschung. Obgleich kein Anlaß besteht, an den vorliegenden Daten zu zweifeln, sei darauf hingewiesen, daß es hier im Interesse der Auftraggeber lag, die Leistungsfähigkeit von regionalen Tageszeitungen als Werbeträger zu untermauern. Zur Abhängigkeit der Glaubwürdigkeit der Werbung von der Glaubwürdigkeit des Werbeträgers siehe auch: David A. Aaker / Phillip K. Brown: Evaluating Vehicle Source Effects, in: Journal of Advertising Research 12/1972, S. 11-16; Lee B. Becker / Raymond A. Martino / Wayne M. Towers: Media Advertising Credibility, in: Journalism Quarterly 53/1976, S. 216-222; Richard M. Durand / Jesse E. Teel, Jr. / William O. Bearden: Racial Differences in Perceptions of Media Advertising Credibility, in: Journalism Quarterly 56/1979, S. 562-566. 
'Objektivität'. Hier ist für alle Medien über die Jahre hinweg ein kontinuierlicher Rückgang zu beobachten. 1990 sind noch 28 Prozent des Publikums der Auffassung, das Fernsehen berichte wahrheitsgetreu (Hörfunk: 24\%; Zeitung: $19 \%){ }^{77}$ Die widersprüchlichen Ergebnisse lassen sich zumindest teilweise dadurch erklären, daß in der Regionalpresse-Studie ein größeres Antwortspektrum vorlag, wobei insbesondere die Option 'Anzeigenblätter' wahrscheinlich zu einer verstärkten Kontrastierung geführt hat. Nicht auszuschließen, da der Fragebogen nicht dokumentiert ist, aber auch nicht zu überprüfen, sind etwaige Halo-Effekte.

Festzuhalten bleibt jedenfalls, daß die Einschätzungen der Rezipienten offenbar stark von der Art der Fragestellung sowie von der Art der einzuschätzenden Medien abhängig sind. Für beide Studien gilt, daß die Fragestellung zu kurz greift, um eindeutige Aussagen über das komplexe Konstrukt 'Glaubwürdigkeit' abzuleiten. ${ }^{78}$

Differenziertere Ergebnisse legt Bentele vor. Er fragt nicht nach Mediengattungen, sondern nach konkreten, namentlich genannten Einzelmedien und nimmt eine ausdrückliche Eingrenzung auf die politischen Nachrichten vor. Leider werden in der Fragestellung die - verwandten aber sicher nicht bedeutungsgleichen - Begriffe 'Glaubwürdigkeit' und 'Objektivität' gekoppelt. ${ }^{79}$

77 Vgl. Berg / Kiefer (1992), S. 254. Vertrauen in die Wahrheitstreue der Berichterstattung als einziges Glaubwürdigkeitskriterium findet sich auch bei Kurt Rolf Hesse: Nutzung und Image des "Westfernsehens" bei DDR-Übersiedlern, in: Media Perspektiven 4/1986, S. 265-272.

78 Die direkte Frage "Für wie glaubwürdig halten sie die Berichterstattung folgender Medien?" erbringt wiederum andere Ergebnisse: Die Optionen "sehr glaubwürdig" oder "eher glaubwürdig" wählten für Zeitungen $72 \%$, Für Hörfunk 83\%, für Privatfernsehen 62\%, für öffentlich-rechtliches Fernsehen 84\%, für Nachrichtenmagazine 79\%.(Vgl. o.V.: Treu und Glauben. Was die Deutschen von Presse, Funk und Fernsehen halten, in: Spiegel special 1/1995, S. 168, unter Berufung auf eine EMNID-Umfrage.)

79

Vgl. Bentele (1988), S. 415. Die Frage lautet: "Ich lese Ihnen gleich noch einmal einige Tageszeitungen, Hörfunk- und Fernsehprogramme vor. Sagen Sie mir bitte jeweils anhand der Liste, für wie glaubwürdig und objektiv Sie diese politischen Nachrichten- und Informationsbeiträge halten. 1 bedeutet dabei, daß Sie die politischen Nachrichten für völlig unglaubwürdig und nicht objektiv halten, 7 hingegen, daß Sie sie für völlig glaubwürdig und objektiv halten. Mit den Werten dazwischen können Sie Ihre Meinung abgestuft abgeben. Wenn Sie das 
Obgleich die bei Berg/Kiefer ermittelte Abstufung der Glaubwürdigkeit von Fernsehen, Hörfunk und Presse tendenziell erhalten bleibt, werden die Abstände zwischen den Medien deutlich kleiner, was wohl auf die konkretere Fragestellung zurückzuführen ist. ${ }^{80}$ Ferner zeigt sich, daß

"intramediale Differenzen in der Glaubwürdigkeit (...) eine offenbar ebenso wichtige Rolle
[spielen] wie die bisher vor allem bekannten intermedialen Differenzen. Ursache und Erklärung
für solche Differenzen in der Glaubwürdigkeit ist offenbar die Tatsache, daß nicht nur die
Verfaßtheit bzw. die technische Struktur der Medien, sondern - teilweise stärker - die politische
Ausrichtigung [sic] der Medien, die Art der Zeitung (Straßenverkaufszeitung versus Abon-
nementszeitung) bei der Glaubwürdigkeitseinschätzung der Medien ins Gewicht fallen. Was die
politische Ausrichtung der Medien betrifft, so werden westliche Medien offensichtlich nicht nur
im Westen als glaubwürdiger eingeschätzt als die DDR-Medien: auch viele Einwohner der DDR
messen den 'westlichen' Medien eine teilweise deutlich höhere Glaubwürdigkeit zu."

Aus der Betrachtung der Einzelmedien lassen sich zwar interpretativ Anhaltspunkte hinsichtlich der für die Beurteilung relevanten Glaubwürdigkeitskriterien ableiten, ein konkreter Nachweis bleibt aber multivariaten Untersuchungsansätzen vorbehalten. Einen solchen Ansatz wählen Deimling et al. Ähnlich wie Gaziano/McGrath legen sie den Befragten ausgewählte Items (insgesamt 18) vor. Die Faktorenanalyse ergibt zwei Faktoren, also zwei Glaubwürdigkeitsaspekte, für die die Bezeichnungen "Informationsqualität" und "Ethik" gewählt werden. "Informationsqualität" beinhaltet Aspekte der Wahrheitstreue, Vollständigkeit der Berichterstattung, Ausführlichkeit, Verständlichkeit sowie Ausgewogenheit und Meinungsvielfalt; ethische Gesichtspunkte manifestieren sich etwa in Aussagen über das Postulat der Trennung von Nachricht und Kommentar, Einflußnahme von seiten der Industrie sowie allgemein die "Grenzen des guten Geschmacks". ${ }^{82}$

An einer konkreteren Operationalisierung der Glaubwürdigkeitsaspekte von

entsprechende Medium zu wenig kennen, sagen Sie das dann bitte jeweils."

80

Vgl. ebd. S. 415.

$81 \quad$ Ebd. S. 416

82 Vgl. Deimling / Bortz / Gmel (1993), S. $212 f$. 
Medien versuchen sich auch Bortz/Leitner: Die Dimension 'Sympathie' gründet sich auf die Beantwortung der Fragen, wie sympathisch bzw. unsympathisch eine Zeitung ist und wie gern bzw. ungern sie gelesen wird. 'Vertrauenswürdigkeit' mißt sich an der Trennung von Nachricht und Kommentar, am Ausmaß von Meinungsäußerungen in Nachrichten sowie an den Gegensatzpaaren 'versucht zu informieren' - 'versucht zu beeinflussen', ist 'emotional getönt' vs. 'rational argumentierend', 'stellt ihre Meinung offen heraus' bzw. 'versucht ihre Meinung zu verschleiern'. 'Sachverstand' wird erhoben anhand der Items 'ist im Urteil einseitig' oder 'ist abgewogen', 'hebt einseitige Gesichtspunkte hervor' bzw. 'versucht zu differenzieren', 'argumentiert glaubwürdig' oder 'unglaubwürdig' sowie 'argumentiert sachlich / unsachlich'. ${ }^{3}$

\subsection{Spezielle Aspekte von Medien-Glaubwürdigkeit}

\subsubsection{Stilistische und inhaltliche Elemente}

Mosier/Ahlgren prüfen, ob eine unterschiedliche Aufbereitung von Informationen die Glaubwürdigkeit beeinflussen kann. Sie präsentieren als Stimulusmaterial Beiträge, die Elemente des "Präzisionsjournalismus" enthalten, solche, die "Pseudo-Präzision" darstellen und solche, die auf "traditionelle" Art verfaßt sind. Die "Präzisions"-Variante beinhaltet zur Stützung allgemeiner Aussagen Datenmaterial aus Umfragen sowie Informationen über die Datenbeschaffung, Stichprobenziehung und Untersuchungssample. Die Daten im "Pseudo-Präzisions"-Artikel werden ohne Quellen und Hinweise auf die Methode präsentiert; zusätzlich werden in dem Beitrag unzulässige oder widersprüchliche Schlußfolgerungen aus dem Datenmaterial gezogen. Der "traditionelle" Beitrag enthält anstelle von Daten Zitate von namentlich genannten und

83 Vgl. Bortz / Leitner (1979), S. 77. Die Einstufung erfolgte jeweils anhand 6-stufiger Ratingskalen. Befragt wurden Fachhochschüler nach einjährigem Freiabonnement einer Tageszeitung. Warum der Dimension 'Sachverstand' hier Ausprägungen zugeordnet werden, die eher zur 'Vertrauenswürdigkeit' zu zählen sind, wird nicht erläutert. 
anonymen Aussageträgern aus verschiedenen gesellschaftlichen Bereichen. ${ }^{84}$

Entgegen den Erwartungen der Forscher, die von einer höheren Glaubwürdigkeit des Präzisionsjournalismus ausgingen, wird keine der drei Varianten durchgehend als glaubwürdiger eingestuft. Bei verschiedenen Themen ergibt sich hinsichtlich der ermittelten Faktoren ('Clarity', 'Accuracy', 'Trustworthiness' und 'Believability') kein konsistentes Bild. Daraus leiten sie die Vermutung $a b$, daß neben dem zur Diskussion stehenden Thema personale Aussageträger von Bedeutung sind: "As sources of information, local people with good reputations carry more credibility than other high ranking officials or any collection of data." 85

Der Einsatz von Umfragedaten beschäftigt auch Salwen. Er prüft, ob "precision poll stories" glaubwürdig sind und ob ein Zusammenhang besteht zwischen der zugewiesenen Glaubwürdigkeit und dem Urheber der Umfrage, der Absicht des Urhebers und dem Verweis auf Stichprobenverfahren. Lediglich bei dem Hinweis auf Verwendung von Zufallsstichproben kann eine Auswirkung auf drei der vier ermittelten Faktoren nachgewiesen werden: 'Trustworthiness', 'Expertise' und 'Objectivity' sind davon beeinflußt. Für die verschiedenen Urheber hingegen ergeben sich keine signifikanten Unterschiede und die angedeutete Parteilichkeit eines Umfrageinstituts berührt lediglich die Einschätzung des Faktors 'Objektivität'. ${ }^{86}$

Vgl. Nancy R. Mosier / Andrew Ahlgren: Credibility of Precision Journalism, in: Journalism Quarterly 58/1981, S. 375-381, 518.

85

Mosier / Ahlgren (1981), S. 381. Zur Bedeutung inhaltlicher Elemente siehe auch: Erica Weintraub Austin / Qingwen Dong: Source v. Content Effects on Judgments of News Believability, in: Journalism Quarterly 71/1994, S. 973-983.

Vgl. Michael B. Salwen: Credibility of Newspaper Opinion Polls: Source, Source Intent and Precision, in: Journalism Quarterly 64/1987, S. 813-819. Zur Glaubwürdigkeit von Umfragedaten vgl. ferner: David L. Paletz et al.: Polls in the Media: Content, Credibility, and Consequences, in: Public Opinion Quarterly 44/1980, S. 495-513; Andrew Kohut: Rating the Polls: The View of Media Elites and the General Public, in: Public Opinion Quarterly 50/1986, S. 1-10; Burns W. Roper: Evaluating Polls with Poll Data, in: Public Opinion Quarterly 50/1987, S. 1016. 


\subsubsection{Elemente der optischen Präsentation}

Wenn die Frage gestellt wird, inwieweit Kameraperspektiven die Wahrnehmung der dargestellten Personen beeinflussen, wird häufig der vertikale Kamerawinkel als Indikator herangezogen. ${ }^{87}$ Geprüft wird, ob die Urteile über eine Person variieren, je nachdem, ob die Kamera in Augenhöhe postiert ist oder die Person in der Draufsicht (Vogelperspektive) oder Untersicht (Froschperspektive) zeigt. Für die wahrgenommene Glaubwürdigkeit der Sprecher ergeben sich widersprüchliche Befunde. Tiemens entdeckt nur geringe Hinweise, daß die Glaubwürdigkeit von der Kameraperspektive abhängig ist: Nur bei einem von drei Sprechern spielt der Kamerawinkel für die Einschätzung eine Rolle; die Untersicht vermittelt hier am besten den Eindruck der Glaubwürdigkeit. ${ }^{88}$ Hingegen finden McCain et al. deutliche Zusammenhänge zwischen Glaubwürdigkeit und Kamerawinkel, wobei die Vogelperspektive generell höhere Glaubwürdigkeitseinschätzungen erbringt. ${ }^{89}$ Die unterschiedlichen Ergebnisse sind zum einen auf unterschiedliche Operationalisierungen von 'Glaubwürdigkeit' zurückzuführen, ${ }^{90}$ zum anderen

87 Für einen allgemeinen Überblick vgl. Hans Mathias Kepplinger / Wolfgang Donsbach: Der Einfluß von Kameraperspektiven auf die Auffälligkeit und wahrgenommene Tendenz sowie die Informationsvermittlung und die Personenwahrnehmung, in: Kepplinger (1987), S. 92-124.

88 Vgl. Robert K. Tiemens: Some Relationships of Camera Angle to Communicator Credibility, in: Journal of Broadcasting 14/1970, S. 483-490. Eine vorteilhafte Wahrnehmung der gefilmten Person bei mittlerer Untersicht berichten auch: Robert E. Beverly / Thomas J. Young: The Effect of Mediated Camera Angle on Receiver Evaluations of Source Credibility, Dominance, Attraction and Homophily. Paper presented at the International Communication Association Convention, Chicago 1978, S. 11, zitiert nach: Kepplinger / Donsbach (1987), S. 98. Die Darstellung aus der Untersicht führt zudem dazu, daß ein Sprecher als aktiver und dominanter wahrgenommen wird. (Vgl. Lee M. Mandell / Donald L. Shaw: Judging People in the News - Unconsciously: Effect of Camera Angle and Bodily Activity, in: Journal of Broadcasting 17/1973, S. 353-362.) 
können verschiedene Kamerawinkel sowie die Präsentation des Filmmaterials im Kontext ausschlaggebend sein; ${ }^{11}$ schließlich kann das Thema oder die Betroffenheit des Zuschauers die Beurteilung beeinflussen. ${ }^{92}$

Den Einfluß der horizontalen Kameraperspektive auf die Glaubwürdigkeit prüfte Baggaley: ein Sprecher wurde als vertrauenswürdiger und sachkundiger eingestuft, wenn er im Halbprofil statt frontal aufgenommen wurde. ${ }^{93}$

Schließlich kann die Wahrnehmung der Glaubwürdigkeit durch weitere Präsentationselemente beeinflußt werden: Die sichtbare Verwendung von Notizen führt etwa dazu, daß ein Sprecher als weniger fair und aufrichtig betrachtet wird. $^{94}$

\subsection{Zwischenbilanz}

Massenmedien werden in diesem Kapitel als Quellen besonderer Art herausgegriffen. Ihre Sonderrolle manifestiert sich zunächst in einer methodischen Herangehensweise, die sich von derjenigen, die bei personellen Quellen verwendet wird, unterscheidet: Im Blickpunkt der Aufmerksamkeit stehen vor allem die Unterschiede zwischen den Mediengattungen Fernsehen, Hörfunk und Zeitung, deren Glaubwürdigkeit häufig durch eine verhältnismäßig direkte Fragestellung - die Ropersche Frage nach der Glaubhaftigkeit konfligierender Versionen in verschiedenen Medien -erhoben wird.

Konstatiert wird dabei regelmäßig ein Glaubwürdigkeitsvorsprung des Fernsehens, der zurückgeführt wird auf die optische Komponente und das Live-Erlebnis für den Betrachter. Kritiker der Roper-Frage verweisen darüberhinaus darauf, daß die Fragestellung das Fernsehen insofern begünstigt, als

91 Vgl. Kepplinger / Donsbach (1987), S. 103. Hier wird im folgenden aufgezeigt, daß für die Wahrnehmung unterschiedliche Winkelzonen eine Rolle spielen, wobei in bestimmten Bereichen bereits kleine Veränderungen des Kamerawinkels die Einschätzung beeinflussen.

92 Vgl. Tiemens (1970), S. 488f.

93 Vgl. Jon Baggeley: Psychology of the TV Image, Westmead 1980, S. $27 \mathrm{ff}$.

$94 \quad$ Vgl. Baggaley (1980), S. 23. 
auch der Aspekt der Personalisierung für die Zuschreibung der Glaubwürdigkeit eine Rolle spielt und bekannte Nachrichtenpräsentatoren vorwiegend im Fernsehen auftreten. Weitere Kritikpunkte an der Vorgehensweise betreffen die mangelnde Realitätsnähe des Verfahrens sowie die allzu pauschale Trennung in Medientypen, die vernachlässigt, daß die Befragten bei ihrer Antwort in der Regel konkrete Einzelmedien oder Sendungen vor Augen haben.

Die Verwendung Semantischer Differentiale zur Untersuchung der Glaubwürdigkeit von Medien bildet einen weiteren methodischen Schwerpunkt und erbringt im wesentlichen ähnliche Dimensionen wie bei der Untersuchung der Glaubwürdigkeit von Quellen. Ein kritischer Einwand gegen das Verfahren ist, daß personenbezogene Beurteilungskriterien als Maßstäbe für die Einschätzung von Institutionen herangezogen werden. Erst in jüngerer Zeit finden Kriterien Verwendung, die gezielt medienspezifische Gegebenheiten berücksichtigen und die sich gleichzeitig den Hauptdimensionen 'Kompetenz und 'Vertrauenswürdigkeit' zuordnen lassen.

Während die quellenorientierte Glaubwürdigkeitsforschung die Beziehung der Sprecher zu den Rezipienten und ihren Einstellungen gründlich durchleuchtet, belassen es die Wissenschaftler, die sich mit der Glaubwürdigkeit von Medien beschäftigen, häufig dabei, die soziodemographischen Merkmale zu erheben. Sie erhalten dabei widersprüchliche Befunde hinsichtlich des Einflusses von Geschlecht, Bildung und Alter der Rezipienten auf deren Einschätzungen. Darüberhinaus wird lediglich vereinzelt geprüft, wie sich das Ausmaß persönlicher Betroffenheit ('Involvement') auf das Zutrauen zur Berichterstattung auswirkt: Ausgeprägtes Interesse an einem Thema (das mit größerem Wissen einhergeht) führt zu einer erhöhten Skepsis gegenüber der Berichterstattung. Die Wahrnehmung von Ähnlichkeiten hinsichtlich der Werthaltungen verstärkt die Glaubwürdigkeit der Medien.

Zum Einfluß von stilistischen und inhaltlichen Elementen der Berichterstattung liegen ebenfalls nur Einzelbefunde vor. Die Frage, welche Rolle die Art der Präsentation spielt, wird lediglich für das Fernsehen gestellt und verengt sich auf Untersuchungen, die den Einfluß der Kameraperspektive beleuchten. Die unterschiedlichen Ergebnisse lassen keine allgemeingültigen Schlußfolgerungen zu. 


\section{Vertrauenssache - Probleme der Glaubwürdigkeit in der vermittelten Kommunikation}

\subsection{Die Rolle der Glaubwürdigkeit im Vermittlungs- und Mitteilungsprozeß}

Wie bereits aus den Definitionen ersichtlich, ist Glaubwürdigkeit eng an Kommunikation gebunden; für Heringer ist sie gar "die Basis, auf der Kommunikation funktioniert; sie ist das kommunikative Urvertrauen, ohne das nichts geht." ${ }^{1}$ Glaubwürdigkeit wird also stets in Beziehung zu einer Person gesetzt, die eine Aussage macht:

"Es ist ein personales Kriterium, das für die persönliche Orientierung unter den Bedingungen von Ungewißheit wichtig, ja unerläßlich ist. Glaubwürdigkeit vermittelt notwendige Orientierungssicherheit in Situationen unvollständiger Information." ${ }^{2}$

Soweit sie im Rahmen direkter Kommunikation betrachtet wird, ist die

1 Hans Jürgen Heringer: "Ich gebe Ihnen mein Ehrenwort". Politik - Sprache - Moral, München 1990, S. 180f.

2 Ulrich von Alemann: Technik und Interesse. Anmerkungen zu Grundbegriffen und Folgerungen für Glaubwürdigkeit von Experten und Gutachten, in: Walter Ch. Zimmerli / Hansjörg Sinn: Die Glaubwürdigkeit technisch-wissenschaftlicher Experten, Düsseldorf 1990, S. 49-66, S. 56. 
Herstellung dieser Beziehung unproblematisch. ${ }^{3}$ Anders, wenn es um Massenkommunikation geht: Der Forschungsüberblick hat gezeigt, daß dort hinsichtlich des Urhebers einer Aussage Unklarheiten bestehen, die sich zunächst in unterschiedlichen Namensgebungen manifestieren. Da ist die Rede von 'Quellen' oder 'Sprechern', von 'Kommunikatoren' oder 'Medien'. Zusätzlich wird das Verständnis dadurch erschwert, daß die Begriffe jeweils unterschiedlich eingesetzt werden, je nachdem, welcher theoretische Blickwinkel den Autoren als Grundlage dient.

So verwenden etwa Hovland/Weiss die Begriffe 'source' und 'communicator' synonym und betrachten in ihrer Untersuchung einen (namentlich genannten) Wissenschaftler, Zeitungen und Zeitschriften, aber auch einzelne Journalisten gleichermaßen als 'Quellen'. ${ }^{4}$ Auch Merten belegt Journalisten, Politiker und Wissenschaftler gleichermaßen mit dem Begriff 'Kommunikator'. ${ }^{5}$ Ein Großteil der Forscher orientiert sich an der direkten Kommunikation und bezieht 'Quelle' und 'Kommunikator' auf Sprecher unterschiedlichster gesellschaftlicher Provenienz, ohne Medien und deren Mitarbeiter zu berücksichtigen. ${ }^{6}$ Andere

Vertreter der Glaubwürdigkeitsforschung stützen sich daher in der Regel auf einfache rhetorik-orientierte Kommunikationsmodelle; vgl. etwa David K. Berlo: The Process of Communication, New York 1960 und James C. McCroskey: An Introduction to Rhetorical Communication, Englewood Cliffs, N.J. 1968. Für einen Überblick siehe auch Infante / Rancer / Womack (1993), S. 33ff.

Vgl. Hovland / Weiss (1951). Das gleiche Verständnis offenbaren Siegel / Miller / Wotring (1969: 118): "Sources may be persons, groups of persons, or even channels through which particular messages are transmitted."

5

Vgl. Klaus Merten: Risikokommunikation - Probleme und Perspektiven, in: Peter C. Compes (Hrsg.): Risiko - subjektiv und objektiv, Wuppertal 1989, S. 17-49.

6

Vgl. z.B. Jack L. Whitehead Jr.: Factors of Source Credibility, in: Quarterly Journal of Speech 54/1968, S. 59-63 (Quellen sind ein Professor und ein Ex-Manager); Elaine Walster / Elliot Aronson / Darcy Abrahams: On Increasing the Persuasiveness of a Low Prestige Communicator, in: Journal of Experimental Social Psychology 2/1966, S. 325-342 (Staatsanwalt vs. verurteilter Krimineller); Bradley S. Greenberg / Gerald R. Miller: The Effects of Low-Credible Sources on Message Acceptance, in: Speech Monographs 33/1966, S. 127-136 (Verkaufsbroschüre bzw. PR-Mitteilung); Murray A. Hewgill / Gerald R. Miller: Source Credibility and Response to Fear-Arousing Communications, in: Speech Monographs 32/1965, S. 95-101 (Professor vs. Schüler); Homer H. Johnson / John A. Scileppi: Effects of Ego- 
betrachten Medien in ihrer Gesamtheit als Quellen bzw. Kommunikatoren ${ }^{7}$, unterscheiden zwischen "personal news sources" (Kommentatoren, Leitartikler) und "impersonal news sources" (Medien als Institution) ${ }^{8}$ oder fassen "news sources" auf als "any real person in television, radio, newspaper, magazines, wire service or book". 9

Die letztgenannte Betrachtungsweise deutet an, daß es im Fall der Massenkommunikation nötig ist zu unterscheiden zwischen dem Medium und den Personen, die in diesem Medium als 'Quelle' in Erscheinung treten. Diesem Umstand wird für gewöhnlich nicht Rechnung getragen. Lediglich vereinzelt wird auf die Diskrepanz hingewiesen:

"Not only have there been few studies of credibility, but many studies have all but ignored the distinction between the mass media and persons as sources. Experiments commonly deal with a single personal source as communicator. The typical mass communication, however, contains both internal and external sources, and Weiss, for one, has shown the need to distinguish between credibility effects of an internal source, e.g., a person originating a message, and an

Involvement Conditions on Attitude Change to High and Low Credibility Communicators, in: Journal of Personality and Social Psychology 13/1969, S. 31-36 (Röntgenexperte vs. 'Quacksalber'); C. Neil Macrae / John W. Shepherd / Alan B. Milne: The Effects of Source Credibility on the Dilution of Stereotype-Based Judgments, in: Personality and Social Psychology Bulletin 18/1992, S. 765-775 (Collegelehrer vs. Hausmeister).

Vgl. z.B. Richard F. Carter / Bradley S. Greenberg: Newspaper or TV: Which Do You Believe? in: Journalism Quarterly 42/1965, S. 29-34; Raymond S. H. Lee: Credibility of Newspaper and TV News, in: Journalism Quarterly 55/1978, S. 282-287; Cornelius Pratt: How Media Credibility Ratings of African and U.S. Students Compare, in: Journalism Quarterly 59/1982, S. 581-587; Bruce H. Westley / Werner J. Severin: Some Correlates of Media Credibility, in: Journalism Quarterly 41/1964, S. 325-335; James Rotton / Brian F. Blake / Richard Heslin: Dogmatism, Trust, and Message Acceptance, in: Journal of Psychology 96/1977, S. 81-88; Daniel M. Wegner et al.: Incrimination Through Innuendo: Can Media Questions Become Public Answers? In: Journal of Personality and Social Psychology 40/1981, S. 822-832; Bortz / Leitner (1979).

8 Vgl. Leslie W. Sargent: Communicator Image and News Reception, in: Journalism Quarterly 42/1965, S. 35-42.

Michael W. Singletary: Components of Credibility of a Favourable News Source, in: Journalism Quarterly 53/1976, S. 316-319, S. 317. 
external source, e.g., a mass medium transmitting a message." 10

Ähnliche Überlegungen stellen Page/Shapiro/Dempsey an, die anhand von Fernsehnachrichten ermitteln, daß Nachrichten, die auf unterschiedliche Quellen zurückgehen, unterschiedliche Auswirkungen auf die "öffentliche Meinung" haben: "...it is probably misleading to talk about 'media effects' per se; it is much better to deal with the effects of the statements and actions of particular kinds of particular actors, as reported in the mass media."11

Berücksichtigt man diese Unterscheidung nicht, gelangt man unter Um-

10 Harvey K. Jacobson: Mass Media Believability: A Study of Receiver Judgments, in: Journalism Quarterly 46/1969, S. 20-28, S. 21. Jacobson - der hier im übrigen einen falschen Quellenbeleg liefert - vollzieht die Trennung zwischen Massenmedien und Quellen eindeutiger als Weiss, auf den er sich beruft. Die einschlägige Passage lautet dort: "... in discussions of the communicator as a factor in the communication process, it has been implicitly assumed that the effective communicator is the 'external' one, to whom the autorship of the total message is attributed. In a typical communication, however, there are often one or more 'internal' communicators, with the same or different credibility ratings as the external one, that are used as sources for relevant data and assertions. With respect to communication effectiveness, an untrustworthy external source might increase the opinion impact of his message by the judicious use of more prestigeful, internal sources." (Walter Weiss: Opinion Congruence with a Negative Source on One Issue as a Factor Influencing Agreement on Another Issue, in: Journal of Abnormal and Social Psychology 54/1957, S. 180-186, S. 180.)

Umrissen ist damit also zunächst nur die Einbettung von Aussagen Dritter in die Mitteilung einer Quelle zur Stützung der Glaubwürdigkeit der letzteren. Im anschließenden Experiment werden als "äußere" Quellen dann aber Medien verwendet, "innere" Quellen sind namentlich genannte "governmental and professional sources". Die Trennung wird ebenfalls vollzogen bei Sharon Lee Hammond: Health Advertising: The Credibility of Organizational Sources, in: Margaret L. McLaughlin (Hrsg.): Communication Yearbook 10, Newbury Park u.a. 1987, S. 613-628. Buckley kommt zu dem Schluß: "...it may be more practical to view the media as a channel of communication rather than as a source." (John E. Buckley: Credibility and Drug Education: A Critique and Reformulation, in: International Journal of the Addictions 24/1989, S. 489-497, S. 490.)

11 B. I. Page / R. Y. Shapiro / G. R. Dempsey: Television News and Changes in Americans' Policy Preferences. Paper presented at the annual meeting of the Midwest Political Science Association, Chicago, Ill. 1984, zitiert nach: Werner J. Severin / James W. Tankard, Jr.: Communication Theories: Origins, Methods, And Uses In The Mass Media, New York/London ${ }^{3} 1992$, S. 157. Das Problem wird auch erkannt von: John B. Adams: The Relative Credibility of 20 Unnamed News Sources, in: Journalism Quarterly 39/1962, S. 79-82. 
ständen zu irreführenden oder mindestens voreiligen Schlußfolgerungen: So etwa Weischenberg, der die Befunde von Stone/Hoyt auf seine eigene Untersuchung überträgt. Stone/Hoyt unterscheiden zwischen quellenorientierten Personen, für die wichtiger ist, wer etwas sagt, und aussageorientierten Personen, die mehr darauf achten, was gesagt wird. Träger der Aussage sind bei diesen Experimenten ausschließlich personale Quellen wie Professoren oder Schüler. ${ }^{12}$ Weischenberg hingegen verwendet den Begriff 'Kommunikator' eingeengt ausschließlich für den Journalisten und gelangt entsprechend zu der Interpretation, daß quellenorientierte Rezipienten insbesondere durch den Journalisten beeinflußt werden, eine Auslegung, die durch die zitierten Befunde in keinster Weise gedeckt ist. ${ }^{13}$ Schließlich steht dieses 'Wer' - eine der zentralen journalistischen W-Fragen - in massenmedialen Inhalten nur in Ausnahmefällen für den Journalisten; dieser ist in erster Linie Vermittler der Aussagen anderer.

Indirekt wird diese Differenzierung von einer Reihe von Forschern getragen, die im Bereich der Persuasionsforschung Zeitungsartikel als Stimulusmaterial verwenden, wobei die untersuchten 'Kommunikatoren' ausschließlich vermittelte Quellen aus unterschiedlichen gesellschaftlichen Bereichen sind. ${ }^{14}$ Explizite Berücksichtigung findet diese Unterscheidung in der zeitungswissenschaftlichen Kommunikationstheorie. Massenkommunikation wird hier definiert als

$12 \quad$ Vgl. Stone / Hoyt (1974a) und (1974b).

13 Vgl. Siegfried Weischenberg: Bei diesen Zeitungsschreibern lese ich alles zweimal. Was Journalistenimages mit Medienwirkungen zu tun haben, in: Heinz Pürer (Hrsg.): Medienereignisse - Medienwirkungen? Salzburg 1985, S. 80-106, S. $102 \mathrm{f}$.

14 Auf Nachrichten und Berichte stützen sich dabei z.B. Adams (1960), Sereno (1968) und Michele Tolela Myers / Alvin A. Goldberg: Group Credibility and Opinion Change, in: Journal of Communication 20/1970, S. 174-179 sowie George Horsley Smith: Beliefs in Statements Labeled Fact and Rumor, in: Journal of Abnormal and Social Psychology 42/1947, S. 80-90. Horai / Naccari / Fatoullah (1974) verwenden Leserbriefe, Mehrley / McCroskey (1970) ein Zitat aus einem Interview mit der 'New York Times'. 
"ein Modus Sozialer-Zeit-Kommunikation, der prinzipiell durch partnerunabhängige Vermittlungssysteme als wechselseitiger Austausch von Kommunikationsrepräsentanten im Rahmen manifester Vermittlungsverfassungen ermöglicht und vollzogen wird." ${ }^{15}$

Während also gängige Massenkommunikations-Modelle davon ausgehen, daß Massenkommunikation ein einseitiger Kommunikationsprozeß ist, der zwischen professionellen Kommunikatoren und einem dispersen Publikum verläuft, ${ }^{16}$ und somit rhetorik-orientierte Modelle der direkten Kommunikation auf die Massenkommunikation übertragen, liegt dem zeitungswissenschaftlichen Ansatz eine differenziertere Vorstellung zugrunde: Zentrales Unterscheidungskriterium ist - wie in der Definition bereits angedeutet - die Trennung von Mitteilungs- und Vermittlungsprozeß. Am Mitteilungsprozeß beteiligt sind 'Ausgangspartner' als Urheber von Mitteilungen und 'Zielpartner' als diejenigen, an die die Mitteilung gerichtet ist. Ausgangspartner und Zielpartner können sowohl einzelne Personen sein, die für sich oder als Repräsentanten für andere stehen, als auch gesellschaftliche Gruppen oder Institutionen. Während Ausgangspartner meist durch Namensnennung eindeutig identifizierbar sind, lassen sich Zielpartner nicht immer konkret bestimmen. Zum einen werden sie häufig nicht explizit genannt, zum anderen sind in der Regel in der Massenkommunikation neben den unmittelbar Angesprochenen noch weitere Zielpartner betroffen, wie etwa fallbezogen zuständige, fachlich tangierte oder allgemein interessierte. Innerhalb des Modells werden diese Partnerrollen auch als Kommunikationsrollen bezeichnet. ${ }^{17}$

Demgegenüber werden die Vermittlungsrollen eingenommen von prinzi-

15 Hans Wagner: Kommunikation und Gesellschaft, Band 1, München 1978, S. 136.

16

Vgl. dazu Hans Wagner: Vermittlungsverfassung in der Massenkommunikation, in: Publizistik 22/1977, S. 5-13. Ein solches Massenkommunikationsmodell findet sich etwa bei Gerhard Maletzke: Psychologie der Massenkommunikation, Theorie und Systematik, Hamburg 1963.

17 Vgl. Wagner (1978), S. 41f. Die Begriffe gehen zurück auf Otto Groth: Die unerkannte Kulturmacht. Grundlegung der Zeitungswissenschaft, Band 1: Das Wesen des Werkes, Berlin 1960 . 
piell autonomen Vermittlungssystemen einerseits und den Rezipienten andererseits. Die Vermittlungssysteme "stellen sich als Funktionseinheiten dar, in denen alle zur Vermittlung gesellschaftlicher Zeit-Kommunikation notwendigen speziellen Vermittlungsleistungen durch Nutzung technischer Medien (M) von professionellen Vermittlern (V) erbracht werden. ${ }^{18}$

Im Unterschied zu den gängigen Modellen, die Massenkommunikation als einseitigen Prozeß auffassen, der nur feed-backs zuläßt, kann hier - ähnlich wie in der direkten Kommunikation - auch in der Massenkommunikation ein Rollentausch stattfinden. Getauscht werden allerdings ausschließlich die Kommunikationsrollen. Die ursprünglichen Zielpartner einer Mitteilung können diese erwidern und werden so zu Ausgangspartnern, die ursprünglichen Ausgangspartner werden zu Zielpartnern der Erwiderung. Falls die Antwort nicht direkt erfolgt, sondern wieder durch Massenkommunikation vermittelt wird, ist sie in der Regel nicht in der gleichen Manifestation des Mediums (etwa in der gleichen Zeitungsausgabe oder Nachrichtensendung) zu finden, sondern erst in einer späteren. Die Periodizität des Mediums wird somit zu einer Voraussetzung des vermittelten gesellschaftlichen Gesprächs. ${ }^{19}$

Die Trennung von Vermittlungs- und Mitteilungsprozeß läßt sich auch historisch nachvollziehen. Das Problem einer Kommunikation auf Distanz führte zu einer schrittweisen Ausdifferenzierung von Teilfunktionen der Vermittlung, die auch im Prozeß der Massenkommunikation erforderlich sind und hier vom Vermittlungssystem übernommen werden. ${ }^{20}$ Im einzelnen sind dies die Partner- und Aussagenselektion - also die Auswahl der Ausgangspartner, deren Mitteilungen vermittelt werden und die Auswahl derjenigen Teile ihrer Aussagen, die vermittelt werden. Hinzu kommt die Transformation, also die den jeweiligen Medienbedingungen angepaßte Umformung dieser

\footnotetext{
18 Wagner (1978), S. 44. [Hervorhebungen im Original.]

19 Vgl. ebd. S. $42 \mathrm{f}$.

20 Zur historischen Entwicklung vgl. Hans Wagner: Rationalisierungsprozesse der Sozialen Kommunikation, in: Politische Bildung 13/1980, S. 3-32.
} 
Aussagen. Neben diesen redaktionellen Leistungen sind die technischen Vermittlungsleistungen Signalproduktion und Distribution zu erbringen. ${ }^{21}$

Nimmt man dieses Modell als Grundlage, so wird die Frage nach der Glaubwürdigkeit an drei Stellen relevant: zum einen geht es um die Glaubwürdigkeit des Mediums und seiner Vermittler, zum zweiten ist für die Orientierung der Rezipienten die Glaubwürdigkeit der versammelten Ausgangspartner bedeutsam und zum dritten kann die Glaubwürdigkeit der Personen, über die gesprochen wird (die also Objekte der Kommunikation sind) bestärkt oder erschüttert werden. Die Unterscheidung von Ausgangspartnern und Kommunikationsobjekten ist insofern nicht ganz trennscharf, als es zwar Personen gibt, die ausschließlich als Objekte und damit passiv an der Kommunikation beteiligt sind, andererseits aber auch als Ausgangspartner im Rahmen ein und desselben Beitrags in beiden Rollen gegenwärtig sein können: indem sie selbst sprechen und indem über sie gesprochen wird.

Wie der Forschungsüberblick verdeutlichte, sind für die Glaubwürdigkeit von Medien und von Quellen ähnliche Faktoren als Auslöser heranzuziehen. Der Fachkompetenz der Quelle, die das Wissen um die Sache betrifft, steht die Vermittlungskompetenz der Medien gegenüber, also der sachgerechte Umgang mit Quellen und deren Mitteilungen. ${ }^{22}$ Hierzu gehört die Beantwortung der sogenannten W-Fragen ebenso wie die Auswahl der relevanten Gesprächspartner und ihrer Aussagen, die korrekte Vorstellung der Ausgangs-

$21 \quad$ Vgl. Wagner (1978), S. 50.

22 Die Ausführungen zur Glaubwürdigkeit von Medien beziehen sich im Hinblick auf die Untersuchungs-Objekte der anschließenden Inhaltsanalyse ausschließlich auf universelle Medien, die hinsichtlich ihres Partner- und Themenspektrums tendenziell unbegrenzt sind. Bei speziellen Medien - etwa Fachzeitschriften - dürfte neben der Vermittlungskompetenz die Einschätzung der Fachkompetenz für die Glaubwürdigkeit durchaus eine Rolle spielen. Bei Medien, die den Kommunikationsinteressen einer bestimmten Gruppe verpflichtet sind (z.B. Parteizeitungen, Schülerzeitungen), könnte der Aspekt der 'Ähnlichkeit' zwischen Medium und Rezipienten von Belang sein. (Zur Unterscheidung von universellen und speziellen Medien siehe die Grothsche Medientypologie - Groth (1960), S. 383-511 sowie Wagner (1978), S. 112ff. und Heinz Starkulla: Marktplätze sozialer Kommunikation. Bausteine einer Medientheorie, München 1993, darin: III. Zeitschriften, S. 125-163.) 
partner, also die Vermittlung aller Informationen über sie, die der Rezipient für seine Orientierung benötigt sowie eine angemessene Transformation der Aussage, die ihren Sinnkern nicht entstellt. Kurz: die Vermittlungskompetenz umfaßt alle gesprächsleitenden Funktionen, die zur Aufrechterhaltung der Sozialen Zeit-Kommunikation nötig sind.

Der Vertrauenswürdigkeit der Quelle - konkretisiert durch Ehrlichkeit und Abwesenheit von Eigeninteressen - entspricht auf seiten der Medien die Forderung nach Fairneß, Unparteilichkeit und Objektivität. Dazu gehört, daß alle Stimmen gleiche Sprechmöglichkeiten erhalten, daß der Inhalt der Mitteilung nicht verfälscht wird und daß die Vermittler das Postulat der Trennung von Nachricht und Kommentar befolgen. ${ }^{23}$ Bentele bemerkt hierzu:

"Die empirisch feststellbare Tatsache, daß in Kaufzeitungen das Prinzip 'keine journalistische Bewertung in Nachrichten' zu einem großen Teil außer Kraft gesetzt ist, trägt vermutlich wesentlich dazu bei, daß die Glaubwürdigkeit dieser Zeitungen im Vergleich mit Qualitätszeitungen sehr gering ist. (...) Vermutlich bildet sich die Glaubwürdigkeit von Zeitungen auch aufgrund der Wahrnehmung solcher Bewertungen in Nachrichtenartikeln. Die Fähigkeit, zwischen (nicht-wertender) Deskription und Bewertung eines Ereignisses zu unterscheiden und

23 Objektivität wird hier im zeitungswissenschaftlichen Sinn verstanden und verlangt damit vom Vermittler, "daß ... was in der Gesellschaft zur Sprache kommt, menschenmöglich vollständig vermittelt wird." Für den Journalisten bedeutet dies "das Bemühen um unparteilichallseitige und trotz medienbedingter Konzentration mitteilungsadaequate Vermittlung." (Wagner (1978), S. 123.) Aus dieser Sichtweise "ergibt sich, vereinfacht betrachtet, daß das Objekt journalistischer Berichterstattung nicht ein Ereignis an sich oder eine Realität an sich ist, sondern in aller Regel eine kommunikative Vorgabe zu irgendwelchen aktuellen Ereignissen in irgendwelchen Wirklichkeitsbereichen. Anders gesagt: Der Journalist ist konfrontiert mit einer Vielzahl meist kontroverser Interpretationen von Ereignissen in der Gesellschaft, die aus unterschiedlichen Sichtweisen, Interessenlagen und Wertüberzeugungen resultieren. Objektive Vermittlung heißt dann zunächst nichts anderes, als eben diese Interpretationsvorgaben in Gestalt von Mitteilungen über und zu Ereignissen aufzunehmen und darzustellen. Objektivität wird so zu einer - nachprüfbaren - Relation der Entsprechung von vorgegebenem Mitteilungsgeschehen und seiner Vermittlung durch den Journalisten. Daher lassen sich auch am Vermittlungsprodukt, nämlich an Nachricht und Bericht, durchaus die objektiven und die (vom Journalisten geprägten) subjektiven Merkmale weitgehend trennen." (Detlef Schröter: Qualität und Journalismus. Theoretische und praktische Grundlagen journalistischen Handelns, München 1995, S. 26f. Zur Auseinandersetzung mit anderen Auffassungen zur Objektivität siehe ausführlich ebd. S. 17-42.) 
die Vermischung zwischen beiden wahrzunehmen, dürfte zur kommunikativen Alltagskompetenz jedes Rezipienten gehören." ${ }^{24}$

Die Dynamik der Quelle, also ihr Auftreten und ihr Sprechverhalten, verlagert sich bei den Medien auf Faktoren wie Aufmachung und Präsentation von Nachrichten. Die Annahme, "daß nicht nur inhaltliche Merkmale, sondern auch formale Merkmale wie Typographie und Layout einer Zeitung oder die formale Gestaltung von Nachrichtensendungen des Rundfunks wichtige Indikatoren ... sind, Glaubwürdigkeitsmuster auszubilden" ${ }^{25}$, erscheint höchst plausibel, wartet allerdings noch auf ihre empirische Bestätigung. Glaubwürdigkeitsdefizite bei Kaufzeitungen könnten allerdings auch auf die fehlende 'Soziale Billigung' zurückzuführen sein. Dies ist ebenso ungeklärt wie die Frage, inwieweit sich Sympathiewerte auf die Glaubwürdigkeit der Medien auswirken.

Die Glaubwürdigkeit der Ausgangspartner in der Massenkommunikation ist abhängig von den Informationen, die der Vermittler seinen Lesern an die Hand gibt. Abgesehen von Einzelfällen gilt, daß das Wissen und die Vorstellungen über Personen, die hier zu Wort kommen, nicht der eigenen Erfahrung entstammen, sondern ebenfalls mittels Massenkommunikation zustandekommen. Soweit es sich um Personen handelt, die häufiger auftreten, entwickeln sich diese Vorstellungen im Laufe der Zeit und in Abhängigkeit von der Mediennutzung, bis sie sich zu einem 'Ruf' verfestigen.$^{26}$ Bei bekannten ebenso wie bei unbekannten Personen brauchen wir aber stets Kriterien, die uns helfen, das Gesagte einordnen zu können. Diese "Vorstellung" der Gesprächspartner, die zu den Aufgaben des Vermittlers gehört, umfaßt neben

24 Bentele (1994), S. 308. [Hervorhebungen im Original.]

$25 \quad$ Bentele (1988), S. 422.

26 Vgl. dazu bereits den frühen Versuch von Annis/Meier, die zeigten, wie die Bildung und Verfestigung des Eindrucks von anfangs unbekannten Personen durch wiederholte Kommentare gesteuert werden kann. (Albert D. Annis / Norman C. Meier: The Induction of Opinion through Suffestion by Means of "Planted Content", in: Journal of Social Psychology 5/1934, S. 65-81.) 
der Namensnennung Hinweise auf Kompetenzmerkmale und sachliche Herkunft (z.B. Nennung von Berufsbezeichnungen, Titeln und Funktionen innerhalb einer Organisationshierarchie) ebenso wie Hinweise auf mögliche (Eigen-)Interessen der Quelle.

Die Einschätzung der Glaubwürdigkeit von Kommunikationsobjekten schließlich gründet sich auf die Aussagen von Vermittlern einerseits und auf die vermittelten Aussagen von Ausgangspartnern andererseits.

\subsection{Die Information über Quellen}

Die Notwendigkeit der Information über die Quellen von Mitteilungen ergibt sich aus der scheinbar banalen Tatsache,

"daß nur ein sehr geringer Teil unseres aktuellen und potentiellen Wissens in unserer eigenen Erfahrung gründet. Die große Masse unseres Wissens besteht aus Erfahrungen, die nicht wir, sondern unsere Mitmenschen, unsere Zeitgenossen oder Vorgänger gehabt haben und die sie uns übermittelten. Wir wollen diese Art von Wissen sozial abgeleitetes Wissen nennen." 27

Bedeutsam wird diese Art der Information für denjenigen Rezipienten, den Schütz idealtypisch als 'gut informierten Bürger' (als Abkürzung für 'den Bürger, der gut informiert sein will') bezeichnet. Dieser Typ grenzt sich einerseits ab vom Typ des 'Experten', dessen Wissen auf ein beschränktes Gebiet begrenzt ist und dessen Ansichten und Urteile sich auf gesicherte Behauptungen stützen und andererseits vom 'Mann auf der Straße', der über ein Wissen von Rezepten verfügt, "die ihm sagen, wie er in typischen Situationen typische Resultate durch typische Mittel zustandebringen kann" und der sich "in allen Angelegenheiten, die nicht mit solchen praktischen Zweken von

27 Alfred Schütz: Der gut informierte Bürger. Ein Versuch über die soziale Verteilung des Wissens, in: ders.: Gesammelte Aufsätze, Band 2: Studien zur soziologischen Theorie, hrsg. v. Arvid Brodersen, Den Haag 1972, S. 85-101, S. 97 f. 
unmittelbarer Bedeutung verbunden sind ... von seinen Gefühlen und Leidenschaften" leiten läßt. ${ }^{28}$ Der 'gut informierte Bürger' steht zwischen diesen beiden Typen und für ihn gilt:

\begin{abstract}
"Einerseits hat er kein Expertenwissen und strebt es auch nicht an; andererseits beruhigt er sich nicht mit der fundamentalen Vagheit des bloßen Rezept-Wissens oder mit der Irrationalität seiner ungeklärten Leidenschaften und Gefühle. Gut informiert zu sein bedeutet ihm, zu vernünftig begründeten Meinungen auf den Gebieten zu gelangen, die seinem Wissen entsprechend ihn zumindest mittelbar angehen, obwohl sie seinem zuhandenen Zweck direkt nichts beitragen." 29
\end{abstract}

Um zu "vernünftig begründeten Meinungen" zu gelangen, ist der 'Bürger, der gut informiert sein will' also zunächst auf Informationen angewiesen. Woher aber weiß er, welche Informationen richtig oder verläßlich sind, da ihm doch gerade das Wissen und die Sachkompetenz des Experten fehlen, die für eine Einordnung und Beurteilung nötig wären? Die Antwort, die Schütz liefert, lautet: "Er muß soviel Wissen wie möglich über den Ursprung und die Quellen der ihm aktuell oder potentiell auferlegten Relevanzen sammeln." ${ }^{30}$ Mit anderen Worten: Der 'gut informierte Bürger' ist zunächst und vor allem angewiesen auf "Information über die Information". Was er

"... zur 'Beglaubigung' der Sachinformationen konkret braucht, sind Informationen über Identität, Herkunft, Kompetenz und Funktion dessen, der sein Wissen zum jeweiligen Informationsgegenstand mitteilt; sodann aber nicht zuletzt Informationen über seinen 'sozialen Ort', d.h. über seine Zugehörigkeiten, über seine Wertposition und seine Interessenbindung." 31

$28 \quad$ Vgl. Schütz (1972), S. 87f.

29 Schütz (1972), S. 88. [Hervorhebung im Original.] Schütz betont hier gleichzeitig den Konstruktionscharakter der Typen und verweist darauf, daß "jeder von uns im täglichen Leben und zu jedem Augenblick gleichzeitig Experte, gut informierter Bürger und Mann auf der Straße [ist], aber jedes davon mit Bezug auf eine andere Wissensregion."

$30 \quad$ Schütz (1972), S. 97.

$31 \quad$ Hans Wagner: Der schlecht informierte Bürger. Zur Phänomenologie des Informationsverhaltens und dessen möglicher Steuerung. Festvortrag zur Akademischen Feier für Heinz 
Diese Vorstellung weist Parallelen auf zu den unterschiedlichen Strategien der Informationsverarbeitung, die Petty/Cacioppo und Chaiken ermittelt haben. ${ }^{32}$ Eine zentrale beziehungsweise systematische Route der Informationsverarbeitung schlägt derjenige ein, der mit dem Thema vertraut ist, der Experte also, während der 'gut informierte Bürger' keine andere Wahl hat, als periphere Hinweise heranzuziehen und die Informationen heuristisch zu verarbeiten.

Insgesamt stellt Schütz, wie er selbst meint "keineswegs erschöpfend", vier Quellen-Typen vor, die nach Kriterien angeordnet sind, die in engem Zusammenhang mit der Glaubwürdigkeit stehen. Die von ihm benannten Typen sind der 'Augenzeuge', der 'Insider', der 'Analytiker' und der 'Kommentator'. Ein Konstruktionskriterium für die Typenbildung ist die Nähe der Quelle zum Kommunikationsgegenstand: Sowohl der 'Augenzeuge' als auch der 'Insider' kennen das, worüber sie reden, aus unmittelbarer Erfahrung. Der 'Analytiker' und der 'Kommentator' dagegen gründen ihre Meinungen und Aussagen auf die Mitteilungen anderer Quellen. Das zweite Konstruktionskriterium ist die Kongruenz mit dem Relevanzsystem des Rezipienten: 'Augenzeuge' und 'Analytiker' haben ein Relevanzsystem, das mit dem des Rezipienten übereinstimmt oder ihm ähnlich ist, während 'Insider' und 'Kommentator' sich an einem differierenden Relevanzsystem orientieren. ${ }^{33}$

Diese Konstruktionskriterien umreißen mithin nichts anderes als diejenigen Dimensionen, die in der Glaubwürdigkeitsforschung unter der Bezeichnung 'Kompetenz' und 'Ähnlichkeit' Verwendung fanden, wobei hier lediglich die Ähnlichkeit hinsichtlich der Sichtweisen und Werthaltungen gemeint ist. Insbesondere für die beiden (Ideal-)Typen, die einem abweichenden Relevanzund Wertsystem folgen, ist dabei zu begründen, was ihre Glaubwürdigkeit ausmacht: Das Vertrauen in den 'Insider' "gründet auf der Annahme, daß er,

Starkulla am 18. Februar 1988, o.O. (unveröffentlichtes Manuskript), S. 9 f.

32 Vgl. Abschnitt II. C. 6c), S. 76ff.

33 Vgl. Schütz (1972), S. 98f. 
weil er das berichtete Ereignis in einem einzigartigen und typischen Relevanzzusammenhang erlebte, 'es besser weiß', als ich es wüßte, wenn ich das gleiche Ereignis beobachtet hätte aber mir nicht der inneren Bedeutung bewußt gewesen wäre." Der Meinung des 'Kommentators' "traue ich, wenn es mir möglich ist, ein genügend klares und präzises Wissen von dem zugrunde liegenden aber abweichenden Relevanzsystem zu erlangen." 34

Der 'gut informierte Bürger' ist also für seine Orientierung angewiesen auf "Informationen zur Sachnähe oder Sachferne sowie zur 'sozialen Lage"' der Quelle. Diese "Vertrauens-Informationen" sind zugleich ausschlaggebend dafür, "ob der Bürger einer gegebenen Sachinformation Zuverlässigkeit, Richtigkeit und Glaubwürdigkeit unterstellen kann oder nicht." ${ }^{35}$

Ferner in Betracht zu ziehen sind die im Rahmen der Glaubwürdigkeitsforschung nur vereinzelt angedeuteten Hinweise auf die 'Mehrheitsmeinung', die die Glaubwürdigkeit eines Sprechers erhärten können. ${ }^{36}$ Schütz spricht in diesem Zusammenhang von "sozial gebilligtem Wissen", das sowohl den eigenen Erfahrungen als auch dem "sozial abgeleiteten Wissen" zusätzliche Bedeutung verleiht. ${ }^{37}$ 'Sozial gebilligtes Wissen' ist Wissen, das "nicht nur vom Informationssucher selbst oder von solchen gesellschaftlichen Partnern oder Gruppen akzeptiert wird, die dasselbe Relevanzsystem teilen, sondern ... [es wird] auch von Gruppen mit differierenden Relevanzsystemen angenommen

\footnotetext{
$34 \quad$ Schütz (1972), S. 99.

35 Wagner (1988), S. 10.

36 Vgl. Kim Giffin: The Contribution of Studies of Source Credibility to a Theory of Interpersonal Trust in the Communication Process, in: Psychological Bulletin 68/1967, S. 104-120, S. 107, der als einziger einen ausdrücklichen Bezug zur Glaubwürdigkeit herstellt. Zum Einfluß der Mehrheitsmeinung siehe ferner Henry T. Moore: The Comparative Influence of Majority and Expert Opinion, in: American Journal of Psychology 32/1921, S. 16-20 sowie Harold Burtt / Don Falkenberg, Jr.: The Influence of Majority and Expert Opinion on Religious Attitudes, in: Journal of Social Psychology 14/1941, S. 269-278. 
oder gar von der gesamten eigenen Gesellschaft anerkannt." ${ }^{38}$ Informationen, die Aufschluß darüber geben, von welchen gesellschaftlichen Gruppen ein bestimmtes Wissen akzeptiert oder abgelehnt wird, haben also ebenfalls eine doppelte Funktion: Sie tragen zur Orientierung und zur Manifestierung von Glaubwürdigkeit bei.

"Eine noch so große Fülle reiner Sachinformationen, gleichgültig, wie konkret, spezifisch oder detailliert sie auch sein mögen, reicht nicht aus, um eine gute Information des Bürgers zu gewährleisten, wenn alle diese Sachinformationen entblößt sind von den sozialen Orientierungsverweisen, von den 'Vertrauensinformationen', welche die Sachinformation erst akzeptabel zu machen oder sie als inakzeptabel auszuweisen in der Lage sind." ${ }^{39}$

Die Offenlegung dieser Quellen ist, wie oben dargelegt, Bestandteil der Vermittlungskompetenz der Medien und trägt wiederum zu deren Glaubwürdigkeit bei. Auch in den einschlägigen Praxis-Handbüchern wird die Notwendigkeit der Quellenkennzeichnung ausdrücklich hervorgehoben:

"Von Nachrichten die Mitteilung der Wahrheit zu erwarten, ist ein unrealistischer Anspruch. Verlangt werden kann aber größtmögliche Genauigkeit und Transparenz der Nachrichtengebung. Dies gilt besonders für die Angaben zur Quelle. Informationen in den Medien müssen nachprüfbar sein. Im Einzelfall kann es sogar notwendig sein, den Leser / Hörer / Zuschauer über die Entstehung einer Information genau zu informieren, damit er sich selbst ein Urteil über ihre Glaubwürdigkeit bilden kann. Die Quelle einer Information muß stets angegeben werden, wenn der Journalist nicht aus eigener Anschauung berichten kann. Die Ausnahme von dieser Regel sind allgemein bekannte Tatsachen (das gestrige Wetter; die Hauptstadt der Bundesrepublik)." 40

38 Hans Wagner: Journalismus I: Auftrag. Gesammelte Beiträge zur Journalismustheorie, Erlangen 1995, S. 199. [Hervorhebung im Original.]

$39 \quad$ Wagner (1988), S. 11.

40 Siegfried Weischenberg: Nachrichtenschreiben. Journalistische Praxis zum Studium und Selbststudium, Opladen ${ }^{2} 1990$, S. 216. Weitere Ausführungen zur Quellentransparenz siehe auch S. 132ff. Der ehemalige dpa-Chefredakteur Hans Benirschke fordert: "Um die Nachricht einordnen zu können, muß die Herkunft deutlich gemacht werden: Das wird in vielen Fällen dem Leser helfen, kritisch zu werten, die Bedeutung zu relativieren. Wenn der Leser erkennt, 


\subsection{Unterschiedliche Vermittlungsstrategien: 'Journalist' und 'Publizist'}

"Wo jedoch liegen die Informationsquellen und aus welchem Grund mag der Bürger sie als ausreichend betrachten, um ihm die eigene Meinungsbildung möglich zu machen?" ${ }^{41}$ Für die Beantwortung dieser Frage ist der Bürger auf die Vermittlungsleistung der Medien angewiesen. Der professionelle Vermittler hat einerseits die Aufgabe, die relevanten Quellen zu suchen und auszuwählen, und andererseits muß er die nötigen 'Vertrauensinformationen' liefern, die die Quelle für den Rezipienten glaubwürdig machen. Ob diese Aufgaben tatsächlich so gelöst werden, daß die für den Rezipienten nötige Orientierung gewährleistet ist, hängt in erheblichem Ausmaß davon ab, welche Vermittlungsstrategie der Vermittler verfolgt. Idealtypisch unterscheiden läßt sich dabei die Position des 'Journalisten' und des 'Publizisten'. ${ }^{42}$

Der 'Journalist' zeichnet sich dadurch aus, daß er

"unparteilich, das heißt unabhängig von einzelnen Kommunikationsinteressen (auch von seinen eigenen!) die Nachrichten (Mitteilungen Dritter) möglichst umfassend und möglichst objektiv in referierenden Berichten (d.h. in medienspezifisch bearbeiteter und in der Regel in hoch konzentrierter Form) vermittelt." 43

daß die Mitteilung, die er vor sich hat, beispielsweise vom Sprecher einer Interessenvereinigung oder einer politischen Partei kommt, wird er die Nachricht so nehmen, wie sie ist: als Information einer Seite." (Zitiert nach: Werner Meyer: Zeitungspraktikum, Percha 1983, S. II,9.) Ähnlich äußert sich Walter von La Roche: Einführung in den praktischen Journalismus, München ${ }^{12} 1991$, S. 82. Ausführlich behandelt ist die Frage des Quellenumgangs auch bei: Peter Zschunke: Agentur-Journalismus. Nachrichtenschreiben im Sekundentakt, München 1994, S. 116-131.

$41 \quad$ Schütz (1972), S. 97.

42 Zu den Wurzeln dieser wissenschaftlichen Typenkonstruktion vgl. Hans Wagner: Medien-Tabus und Kommunikationsverbote, München 1991, S. 51f. sowie ausführlich Wagner (1995), 2. Kapitel: Der Ur-Journalist, S. 69-124.

43 Hans Wagner: KommunikationsWissenschaft (ZeitungsWissenschaft), München ${ }^{2} 1989$, S. 54f. 
Er versteht sich also als 'Gesprächsanwalt der Gesellschaft' (Aswerus). Die seiner Verantwortung unterliegenden Vermittlungsleistungen der Selektion und Transformation erledigt er somit mit menschenmöglicher Neutralität. Er trachtet danach, daß alle für das jeweils zur Debatte stehende Thema nötigen Kommunikationspartner zu Wort kommen und keine Position von vorneherein aus dem Gespräch ausgeschlossen wird, bemüht sich also um "allseitige Fremdvermittlung". ${ }^{44}$ Bei der Auswahl der Partneraussagen und deren Transformation achtet er sorgfältig darauf, daß ihr Sinngehalt nicht verfälscht wird, bemüht sich also um "Mitteilungsadäquanz". ${ }^{45}$ Die Vermittlung seiner eigenen Position kann in diesem Zusammenhang nur zusätzlich geschehen und erfolgt, strikt von der referierenden Berichterstattung getrennt, in kommentierenden Darstellungsformen. Damit "tritt er sichtbar aus der Rolle des Journalisten heraus und übernimmt die des Publizisten." ${ }^{46}$

Dessen Rolle ist entsprechend "dadurch bestimmt, daß die subjektiven Einstellungen und Meinungen des Publizisten als Auswahl- und Vermittlungsstrategien fungieren. Vermittlungsprinzip ist also Parteilichkeit (im weitesten Sinn)." ${ }^{47}$

'Publizist' ist damit jeder Gesprächspartner, der (eigene oder fremde) Interessen vertritt. "Der 'Publizist' ist Meinungsgeber oder Meinungsrepräsentant". ${ }^{48}$ Neben denen, die in eigener Sache sprechen oder sich zum Anwalt

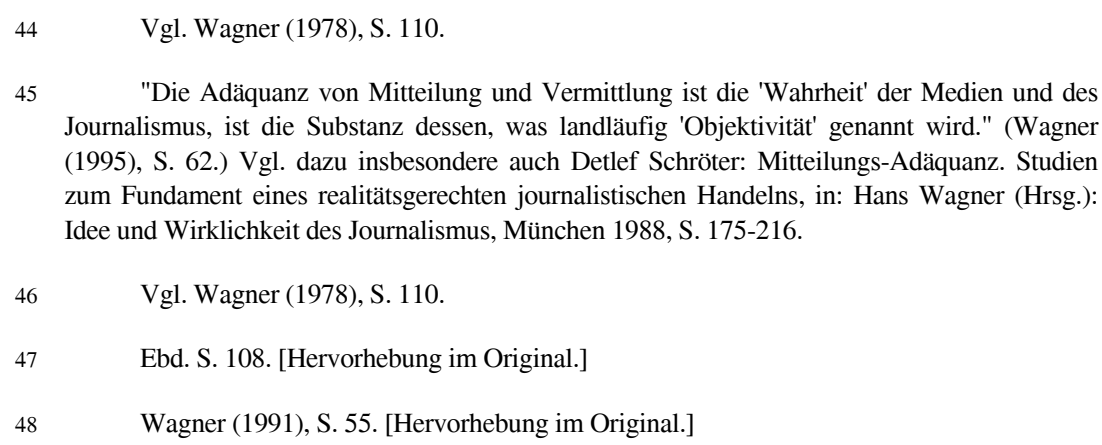


von unorganisierten Interessen machen, haben sich zur Optimierung von Artikulationschancen Berufsfelder professioneller Publizisten herausgebildet, die in den Bereichen Werbung und Public Relations tätig sind oder für Parteiblätter, Gruppen- und Interessenmedien arbeiten. ${ }^{49}$

Mit der idealtypischen Unterscheidung von 'Journalist' und 'Publizist' ist zunächst noch keine Wertung verbunden, da im Rahmen Sozialer Zeit-Kommunikation beide Funktionen nötig sind: Der 'Publizist' "spielt in der Gesellschaft eine unverzichtbare Rolle: Er vereinigt auf seine Weise die Gleichgesinnten zu Gruppen, er bestärkt deren Zusammenhalt, er vertritt deren Interessen nach außen." ${ }^{50}$ Während der 'Publizist' also "Sprechinstanz" ist, ist der 'Journalist' "Gesprächsinstanz". ${ }^{51}$

"Publizisten in allen Erscheinungsformen markieren die zu vermittelnden Gesprächspositionen in der Gesellschaft, nicht zuletzt und insbesondere die nicht organisierten, informellen Meinungspositionen. Der Journalist jedoch ermöglicht erst die 'Versammlung' der publizistisch sich artikulierenden Positionen zum Gespräch aller, damit die Vernehmbarkeit jeder Position für alle und schließlich so die Transparenz des Kommunikationsraumes." ${ }^{52}$

$\mathrm{Zu}$ kritisieren allerdings ist der "Publizist am falschen Platz" ${ }^{53}$, also der Vermittler, der publizistische Vermittlungsstrategien auf Kosten der journalistischen Leistungen verfolgt:

"In der Praxis müßten Kommunikationsakt und Vermittlungsakt nicht nur präzise unterschieden, sondern sauber voneinander geschieden werden. Wenn der Mittler an dieser Scheidung nicht mehr festhält, schiebt er sich als neuer, eigenständiger, ungebetener Partner in den Hauptakt der Kommunikation, den er eigentlich professionell zu vermitteln hätte. Genau damit beginnt die

$\begin{array}{ll}49 & \text { Vgl. ebd. S. 55f. sowie Wagner (1989), S. 52ff. } \\ 50 & \text { Wagner (1991), S. 53. } \\ 51 & \text { Vgl. Wagner (1995), S. } 161 . \\ 52 & \text { Wagner (1978), S. } 110 . \\ 53 & \text { Wagner (1991), S. } 61 .\end{array}$


Störung und Zerstörung des Hauptdialogs: Der Mittler reißt ihn an sich, überdeckt ihn und löst ihn schließlich auf. Das geschieht, wo immer und sobald immer ein 'publizistisches' Führungsprogramm das von der Sache her geforderte 'journalistische' Vermittlungsprogramm überlagert." ${ }^{54}$

Solche Einflußnahme kann im Rahmen der Partnerselektion dadurch geschehen, daß der 'Publizist' einseitig vermittelt, also nur diejenigen Positionen auswählt, mit denen er sich identifiziert, oder daß er partiell vermittelt, indem er die Positionen verschweigt, die ausdrücklich seiner Einstellung zuwiderlaufen. Partielle Vermittlung ist schwerer erkennbar, da der "Anschein von Vielstimmigkeit" gewahrt bleibt. ${ }^{55}$ Ferner besteht die Möglichkeit, daß nominell zwar alle Partner zu Wort kommen, daß aber bei der Selektion ihrer Aussagen manipuliert wird. ${ }^{56}$ Das kann dadurch geschehen, daß zentrale Argumente der Meinungsgegner unterschlagen werden oder daß die Argumente 'versteckt' werden, etwa durch untergeordnete Plazierung oder geringe Raumanteile, während die Argumente der präferierten Position wiederholt, umfangreicher und an auffälliger Stelle präsentiert werden.

"Vollends undurchschaubar für den Rezipienten wird indessen eine Informations-Verengung dann, wenn die Wert-Kriterien des 'Publizisten' dafür, was im Einzelfall gut, nützlich und dienlich oder was umgekehrt gefährlich, schädlich oder schlecht ist, zu heimlichen und verheimlichten Selektionskriterien für die zu vermittelten Fakten werden. Die Anwendung solcher Vermittlungstechnik kann dann auf offene Einflußnahme, auf deklarierte 'publizistische'

54 Wagner (1995), S. 171. [Hervorhebung im Original.]

55

Vgl. Wagner (1978), S. 109. Zu dieser vorgetäuschten Vielstimmigkeit bemerkt NoelleNeumann: "Es gibt in der Publizistik so etwas wie eine Feldstärke, eine Relation von 6 : 2 oder $7: 3$, die dem mehrheitlich vertretenen Argument überhaupt erst seine Würze gibt, das kontroverse Element zumischt, das erforderlich ist, um für eine Sache auf die Barrikaden gehen zu können." (Elisabeth Noelle-Neumann: Die Entfremdung. Brief an die Zeitschrift 'Journalist', in: Hans Mathias Kepplinger (Hrsg.): Angepaßte Außenseiter. Was Journalisten denken und wie sie arbeiten, Freiburg/München 1979, S. 261-280, S. 272.) Objektivitätsforderung" verstanden werden. (Wagner (1978), S. 123.) 
Einsprüche völlig verzichten. Die Information kommt dem Rezipienten im biederen Gewand scheinbar sauberer Nachrichten, Referate und Faktendarstellungen ganz unverfänglich entgegen. Daß es sich dabei lediglich um einsinnig ausgewählte Aspekte, um sorgfältig präparierte, einseitige Sichtweisen ohne Alternativen, um kontextgelöste oder durch entsprechende Montagen neu und irreführend kontextierte Sachverhalte handelt, kann zumindest ein Durchschnittsbürger kaum mehr erkennen; am wenigsten dann, wenn die fragliche (und fragwürdige) Information komplexe, für ihn unbekannte, durch eigene Erfahrung nicht erschließbare Sachverhalte betrifft."

Bei der Transformation der Aussagen besteht darüberhinaus die Möglichkeit, sie sinnentstellend oder -verfälschend, unklar oder mißverständlich zu formulieren. Hinzu kommt, daß der Publizist die Möglichkeit hat, durch manifeste Wertungen zu beeinflussen, etwa durch "wertende Überschriften und Vorspannbeiträge" oder durch "Partner- und/oder Aussagewertungen innerhalb von Fremdvermittlungen". ${ }^{8}$

Dort, wo publizistische Vermittlungsstrategien den journalistischen Auftrag überlagern, gerät die Gesellschaft in Gefahr, da Orientierungsmöglichkeit und Urteilsfähigkeit ihrer Mitglieder eingeschränkt sind, wenn sie nicht umfassend informiert werden:

"Auf Grund von 'publizistischer Information' durch interessengebundene und -gesteuerte Beeinflussung, ohne die Möglichkeit durch öffentlich vernehmbare Erwiderung in der Gesellschaft sich selbst zur Geltung zu bringen, wird der Einzelne (potentiell: jeder Einzelne), weil mit seinen Zeitgenossen nicht in Kommunikation, sich selbst überlassen, isoliert. Publizistische Informationen zerreißt [sic] die Gesellschaft in vor sich hinmonologisierende Einzelne und Gruppen, die durch kein Gespräch zueinander finden, sondern durch monopolistische oder quasi-monopolistische Interessen-Institutionen 'in Form gebracht' - einseitig informiert oder zwangsläufig nur an den eigenen Interessen orientiert - zur politischen Tat schreiten." 59

57 Wagner (1995), S. 138. [Hervorhebung im Original.]

$58 \quad$ Wagner (1978), S. 124

59 Heinz Starkulla: Publizistik und Kommunikation. Ein Beitrag zur wissenschaftlichen Erkenntnis der kommunikativen Wirklichkeit, in: Festschrift für Hanns Braun, Bremen 1963, S. 158-167, S. 161f. [Hervorhebung im Original.] 


\subsection{Die Zuschreibung von Glaubwürdigkeit als publizistische Strategie}

Inwieweit tragen nun diese Vermittlungsstrategien zur Aufrechterhaltung oder Zerstörung von Glaubwürdigkeit bei? Als 'Journalist' muß dem Vermittler daran gelegen sein, bei der Partnervorstellung alle für die Orientierung relevanten Informationen bezüglich Herkunft, Kompetenz und Interessenlage zu liefern; eigene Einschätzungen und Wertungen müssen dabei unterbleiben. Der 'Publizist' hingegen kann seine Beeinflussungsabsicht bereits bei der Präsentation dieser 'Vertrauensinformationen' wirken lassen, indem er Informationen unterschlägt, eigene Wertungen vornimmt oder Gewährsleute zitiert, die ihrerseits Einschätzungen vornehmen, die die Argumentationslinie des Publizisten stützen. ${ }^{60}$

Wie sehr die Einschätzungen eines Lesers bereits mittels der PartnerVorstellung geleitet werden können, sei am folgenden, besonders prägnanten Beispiel demonstriert:

"Mannesmann-Chef Werner Dieter, 64, wohnt dort schon über zwei Jahrzehnte. Bei den Bewohnern gilt der erfolgreiche Manager, der den Düsseldorfer Elektronik-, Röhren- und Maschinenbaukonzern seit 1985 leitet, als Ehrenmann und freundlicher Bürger.

Doch genau das ist er offenbar nicht. Der Vorstandsvorsitzende, gefürchtet wegen seines herrischen Führungsstils, hat mit bemerkenswerter Chuzpe seine Macht genutzt, um private Vermögensbildung zu betreiben. (...) Daß der eindeutige Interessenkonflikt Aktionären, Mitarbeitern und Aufsichtsräten nicht gefallen konnte, war Dieter offenbar bewußt. Er ist deshalb nicht als Eigentümer seiner Familienfirma aufgetreten, sondern hat sich versteckt hinter Strohmännern. Wenn schon das Gewissen des ehrbaren Managers ihn nicht besonders plagte, so hat er wohl doch ein kräftig entwickeltes Gefühl dafür, daß dunkle Geschäfte nicht ans Licht der Öffentlichkeit sollten. (...) Mannesman ... hat - so berichten Mitarbeiter - der Hydac-Gruppe des Chefs Milliarden-Aufträge verschafft. Manager in der Konzernzentrale schätzen, Dieter habe so Millionen auf die eigenen Konten gelenkt - ein Fall von Selbst-

60 Die hier skizzierte begriffliche Unterscheidung wird im Verlauf der Arbeit beibehalten: Als 'Journalist' wird stets bezeichnet, wer als professioneller Vermittler unabhängig von Kommunikationsinteressen agiert, als 'Publizist' derjenige, dessen Vermittlungsprinzip Parteilichkeit ist. Die berufliche Tätigkeit, die umgangssprachlich mit der Bezeichnung "Journalist" versehen wird, wird hier mit dem Terminus 'Vermittler' belegt. 
bedienung in der Chefetage. Dubios ist die Raffkementalität des Spitzenmanagers und Kanzlerberaters allemal, einmalig die dreiste Verquickung von Amt und Privatinteressen, die möglicherweise den Staatsanwalt interessieren wird." 61

Zweifellos liefert der Spiegel seinen Lesern mit dieser ausführlichen Beschreibung einige Orientierungsmarken: Neben Namensnennung und Alter werden zunächst eine Reihe von Kompetenz- und Statusmerkmalen mitgeteilt ("Chef", "Vorstandsvorsitzender", "erfolgreicher Manager", "leitet den Konzern seit 1985", "Spitzenmanager", "Kanzlerberater"). In Sachen Kompetenz und Erfahrung ist die Quelle also hervorragend ausgewiesen. Ganz anders in den Dimensionen 'Vertrauenswürdigkeit' und 'Sympathie': Zwar erhalten wir von (anonym bleibenden) Bewohnern seines Wohnortes die Einschätzungen "Ehrenmann" und "freundlicher Bürger"; diese werden aber sofort widerlegt und überlagert von einer Fülle gegenteiliger Charakterisierungen, die größtenteils vom Publizisten selbst stammen (in ihrer Deutlichkeit aber ebensogut einem der Experimente im Rahmen der Persuasionsforschung entnommen sein könnten): Der Mann ist nur auf seinen eigenen Vorteil bedacht ("hat seine Macht genutzt, um private Vermögensbildung zu betreiben", "eindeutiger Interessenkonflikt", "Raffkementalität", "dreiste Verquickung von Amt und Privatinteressen"), dazu ist er hinterhältig ("hat sich hinter Strohmännern versteckt" und trachtet danach, daß "dunkle Geschäfte nicht ans Licht der Öffentlichkeit sollten") und wahrscheinlich sogar kriminell (sein Tun "wird möglicherweise den Staatsanwalt interessieren"). Die Mischung aus Wertungen und Vermutungen wird komplettiert durch Aussagen ausschließlich anonymer Quellen aus dem beruflichen Umfeld.

Im Anschluß an diese Einführung versichert der Konzernchef, daß sich seine Geschäfte im Rahmen des Üblichen bewegen, daß er dafür eine offizielle Genehmigung hat und daß seine Beteiligungen den zuständigen Gremien seit 1968 bekannt sind. ${ }^{62}$ Daß die Zahl der Leser, die dieser Aussage unter dem

$\begin{array}{ll}61 & \text { Spiegel 24/1994, S. } 96 . \\ 62 & \text { Vgl. Spiegel 24/1994, S. } 97 .\end{array}$


Eindruck der zahlreichen 'Mißtrauens-Informationen' noch Glauben schenken mag, eher gering sein wird, steht, wenn man sich die Ergebnisse der einschlägigen Experimente in Erinnerung ruft, wohl außer Frage; gleichzeitig ist den Lesern die Möglichkeit zur Orientierung nahezu vollständig genommen:

"Mit Hilfe selektiv arrangierter Vertrauensinformationen lassen sich Sachinformationen zu 'sozial gebilligten' Realitäts-Sichten aufwerten und entgegenstehende Realitäts-Sichten als bedeutungslos, unsinnig oder unvernünftig abwerten. Das Arrangement generiert einen binären Schematismus von Aufwertung und Abwertung als Orientierungsrahmen.

Die Verkürzung von spezifischen, konkreten und detaillierten Sachinformationen auf diffuse Sach-Typifikationen oder Attributionsbündel kann Vertrauensinformationen nicht ersetzen; fehlen letztere insbesondere bei der Darstellung neuer, bislang unbekannter Situationen, leistet das Verfahren stets der Täuschung des Bürgers Vorschub." 63

Insbesondere bei Personen oder Personengruppen, die nicht häufig als Ausgangspartner in Erscheinung treten, wird die Vertrauens-Information bedeutsam. Dies gilt etwa, wenn technische oder wissenschaftliche Belange Gegenstand der Berichterstattung sind. Ein typisches Beispiel ist die Debatte um die Sicherheit von Atomkraftwerken oder - in neuerer Zeit verstärkt - um Nutzen oder Schaden der Gentechnologie. Seit dem Reaktorunfall in Tschernobyl 1986 erfährt das Feld "Risikokommunikation" gesteigerte Beachtung; der Glaubwürdigkeit der beteiligten Kommunikationspartner wird dabei verstärkte Bedeutung beigemessen. ${ }^{64}$ Für die an der Debatte beteiligten Politiker ist der

63 Wagner (1988), S. 23.

64 Für die Öffentlichkeitsarbeit in Krisensituationen existieren eine Reihe von Anleitungen, wie die Glaubwürdigkeit gestärkt werden kann. Siehe dazu etwa: Frank M. Ruff: Risikokommunikation als Aufgabe für die Umweltmedizin, in: Karl Aurand / Barbara P. Hazard / Felix Tretter (Hg.): Umweltbelastungen und Ängste. Erkennen. Bewerten. Vermeiden, Opladen 1993, S. 327-364; Peter M. Wiedemann: Öffentlichkeitsarbeit bei Krisen: Ein Leitfaden zur besseren Kommunikation, Eschborn 1990; B. J. Hance / C. Chess / P. M. Sandman: Improving Dialogue with Communities: A Risk Communication Manual for Government, in: Vincent T. Covello / David B. McCallum / Maria T. Pavlova (Hg.): Effective Risk Communication. The Role and Responsibility of Government and Nongovernment Organizations, New York/London 1989, S. 195-285. Zur Bedeutung der Glaubwürdigkeit siehe Ortwin Renn / Debra Levine: Credibility and Trust in Risk Communication, in: Helmut Jungermann / Roger Kasperson / Peter M. 
Kennzeichnungspflicht meist dadurch Genüge getan, daß ihre Parteizugehörigkeit mitgeteilt wird. Diese Information ermöglicht dem Rezipienten die Zuordnung der Meinungsposition zu einem bestimmten Lager und gibt ihm Aufschluß über deren soziale Billigung. Anders liegt der Fall bei den Stimmen, die von seiten der Wissenschaft in die Diskussion eingeführt werden. Jeder aufmerksame Rezipient wird feststellen können, daß ihrer Herkunft nach gleichermaßen kompetente "Experten" konträre Positionen einnehmen; da es ihm selbst an Sachkenntnis fehlt, die Stichhaltigkeit ihrer Argumentation beurteilen zu können, ist es ihm nicht zu verübeln, wenn er sich hilflos oder verwirrt von der Debatte abwendet. ${ }^{65}$ Diesen Umstand kritisiert auch Wagner, bezogen auf die Tschernobyl-Berichterstattung in der Bundesrepublik Deutschland:

"Da wurden tausende von Detailinformationen aneinandergereiht - und es wurde, aufs ganze gesehen doch denkbar schlecht informiert. Was nämlich sollten Nicht-Experten wohl mit all diesen expertenhaft spezifischen Informationen anfangen können? Da wurden sodann dutzende von 'Experten' vorgeführt; sicherlich, sie waren als 'Experten' erkennbar, das heißt, ihre Nähe oder Ferne zur Sache, ihre berufliche Herkunft, ihre förmliche Kompetenz war nachgewiesen,

Wiedemann (Hg.): Themes and Tasks of Risk Communication. Proceedings of the International Workshop on Risk Communication held at the KFA Jülich, October 17-21, 1988, Jülich 1988, S. 51-81. Zur Rolle der Medien: Georg Ruhrmann: Risikokomunikation, in: Publizistik 37/1992, S. 5-24; Judith Lichtenberg / Douglas MacLean: The Role of Media in Risk Kommunication, in: Jungermann / Kasperson / Wiedemann (1988), S. 33-48; Lee Wilkins / Philipp Patterson: Risk Analysis and the Construction of News, in: Journal of Communication 37/1987, S. 80-92. Zur Risikokommunikation allgemein siehe ferner: Merten (1989), Roger E. Kasperson / Peter Jan M. Stallen (Hg.): Communicating Risks to the Public. International Perspectives, Dordrecht 1991; Helmut Jungermann / Bernd Rohrmann / Peter M. Wiedemann (Hg.): Risiko-Konzepte, Risiko-Konflikte, Risiko-Kommunikation, Jülich 1990; Jens Krüger / Stephan Ruß-Mohl (Hg.): Risikokommunikation. Technikakzeptanz, Medien und Kommunikationsrisiken, Berlin 1991.

65 Dabei ist nicht nur die Bezeichnung "Experte" für die Beurteilung von Rezipienten ausschlaggebend. BRASKAMP et al. zeigen, daß allein die Benennung "Forscher" oder "Gutachter" bereits zu höheren Objektivitätsratings führt. (Vgl. Larry A. Braskamp / Robert D. Brown / Dianna L. Newman: The Credibility of a Local Educational Program Report: Author Source and Client Audience Characteristics, in: American Educational Research Journal 15/1978, S. 441450, S. 448f.) 
mitunter sogar ganz nachdrücklich unterstrichen. Jedoch blieb in zahllosen Fällen ihre 'soziale Lage' sorgfältig verborgen: Über ihre Wert-Stellung, über ihre Interessenposition, über ihre Zugehörigkeit zu entsprechenden sozialen 'Lagerungen' erfuhr der Bürger nichts..." 66

Dieses Verschweigen von Vertrauensinformation kann Bestandteil einer publizistischen Vermittlungsstrategie sein. Kepplinger berichtet im Zusammenhang mit der Kernenergie von einer "instrumentellen Aktualisierung" der Expertenaussagen durch die Vermittler: Er sieht einen "klaren Zusammenhang zwischen der redaktionellen Linie der Kernenergieberichterstattung der untersuchten Blätter und der Tendenz der Expertenaussagen", die "bewußt oder unbewußt zur Stützung der redaktionellen Linie publiziert" wurden. ${ }^{67}$ Bei der Berichterstattung über die Diskussion im Vorfeld der Volkszählung 1987 werden zur Stützung der redaktionellen Linie ebenfalls bevorzugt "opportune Zeugen" vermittelt. ${ }^{68}$

Peters spricht im Falle der Tschernobyl-Berichterstattung gar von einem

Kepplinger unterläßt es allerdings, diesen Befund durch die Angabe konkreter Fallzahlen zu manifestieren. (Vgl. Hans Matthias [sic] Kepplinger: Kernenergie. Berichterstattung in ausgewählten Printmedien, Erlangen 1989, S. 23). Eine indirekte Bestätigung dieses Befundes liefert auch Haller: Seine Inhaltsanalyse der Tschernobyl-Berichterstattung, die sich auf Sachund Deutungsfehler konzentriert, erbringt, daß die "industriefreundlichen" Zeitungen 'Frankfurter Allgemeine' und 'Neue Zürcher Zeitung' vermehrt solche Fehler produzierten oder transportierten, die den Schaden für die Bundesrepublik Deutschland als eher gering erscheinen ließen, während die 'Frankfurter Rundschau' häufiger Fehler enthielt, die die Schäden dramatisierten. (Vgl. Michael Haller: Wie wissenschaftlich ist Wissenschaftsjournalismus? Zum Problem wissenschaftsbezogener Arbeitsmethoden im tagesaktuellen Journalismus, in: Publizistik 32/1987, S. 305-319, S. 310.) Möglicherweise steht dieser Befund in Zusammenhang mit einem anderen, der besagt, daß etwa Informationen über Reaktorsicherheit oder Strahlenrisiken überwiegend von Quellen aus dem politisch-administrativen Bereich stammen und nicht von wissenschaftlichen Quellen. (Vgl. Will Teichert: Tschernobyl in den Medien. Ergebnisse und Hypothesen zur Tschernobyl-Berichterstattung, in: Rundfunk und Fernsehen 35/1987, S. 185204.)

68

Vgl. Lutz M. Hagen: Die opportunen Zeugen. Konstruktionsmechanismen von Bias in der Zeitungsberichterstattung über die Volkszählungsdiskussion, in: Publizistik 37/1992, S. 444-460. 
"information disaster" 99 , während Renn die Medien von Schuld freispricht:

"The coverage of overreaction and the emphasis on inconsistencies between official recommendations were additional causes of public discomfort and scepticism. But all these phenomena were real and not invented by the media. Overlapping responsibilities, contradictive advice, inability to explain the meaning of specific countermeasures, and total chaos in the units and intervention levels characterized the European scene. Media coverage was merely a reflection of what actually happened in most countries." 70

Diese Einschätzung greift allerdings zu kurz. Gerade, wenn solch widersprüchliche Informationen und Ratschläge vorliegen und pflichtgemäß vermittelt werden, kommt den Vertrauensinformationen besondere Bedeutung zu. Die Analyse von Peters' Befragungsdaten erhärtet den Schluß, daß gerade vermutete Eigeninteressen von Quellen deren Glaubwürdigkeit beeinflussen und - im Fall der Kernforschungszentren und der Nuklearindustrie - deren hohe Kompetenz überlagern. ${ }^{71}$

69 Vgl. Hans Peter Peters: The Credibility of Information Sources in West Germany after the Chernobyl Disaster, in: Public Understanding of Science 1/1992, S. 325-343, S. 325. Siehe ferner: Helmut Lukesch / Karl-Heinz Kischkel / Wolfgang Nöldner: Zur Beurteilung von Informationsquellen und Medien im Zusammenhang mit dem Reaktorunfall von Tschernobyl, in: Publizistik 32/1987, S. 154-158; Hans Peter Peters et al.: Die Reaktionen der Bevölkerung auf die Ereignisse in Tschernobyl. Ergebnisse einer Befragung, in: Kölner Zeitschrift für Soziologie und Sozialpsychologie 39/1987, S. 764-782; Hans Peter Peters / Leo Hennen: Orientierung unter Unsicherheit. Bewertung der Informationspolitik und Medienberichterstattung nach 'Tschernobyl', in: Kölner Zeitschrift für Soziologie und Sozialpsychologie 42/1990, S. 300-312. Hier wird ebenfalls "unterschieden zwischen den Primärquellen von Informationen, wie etwa Bundes- und Landesregierung, Forschungsinstitute, Umweltverbände und Bürgerinitiativen und den Massenmedien, die als Vermittler der Aussagen dieser Primärquellen fungieren und deren Einfluß auf das Informationsangebot im wesentlichen in der Auswahl, Darstellung und Kommentierung der vermittelten Informationen bestand." (S. 301)

Ortwin Renn: Public Responses to the Chernobyl Accident, in: Journal of Environmental Psychology 10/1990, S. 151-167, S. 160.

71 Vgl. Peters (1992), S. 334f. Generell ergaben sich geringe Unterschiede bezüglich der Glaubwürdigkeit der vorgegebenen Quellen, was möglicherweise zum einen daran liegt, daß größtenteils nicht einzelne Personen oder Institutionen, sondern Aggregate zur Bewertung gestellt wurden (die Vorgaben waren: Regieung, Opposition, Kernforschungszentren, ÖkoInstitut, Kernindustrie, Anti-Atom-Bürgerinitiativen und Journalisten) und zum anderen daran, 
Zudem kann er bestätigen, daß die Wahrnehmung der Glaubwürdigkeit signifikant von den Einstellungen der Rezipienten abhängt: Personen, die der Kernenergie kritisch gegenüberstehen, halten solche Quellen für glaubwürdiger, die diese kritische Haltung teilen. ${ }^{72}$ Solche individuellen Präferenzen entlasten den Vermittler aber nicht von seiner Aufgabe, umfassend und allseitig zu informieren, denn:

"... als Typus ist dieser [i.e. der gut informierte Bürger] ja nur bestimmt ... durch die Typik seines Informationsverhaltens... Typisch für ihn ist also nur und ausschließlich, daß er in dieser Lage genötigt ist, die Entscheidung darüber, welche Sachinformationen er als zuverlässig genug für sein eigenes Urteilen und Handeln wahrnehmen und verarbeiten will, von der Auswertung aller beigegebenen und entschlüsselbaren 'Vertrauensinformationen' abhängig zu machen. Welche der je angebotenen Realitäts-Sichten samt ihrer in den Vertrauensinformationen manifestierten sozialen Verortung dabei für jeden einzelnen Bürger, der gut informiert sein will, infrage kommen, ist aber gerade nicht ein Kriterium dieser Typenkonstruktion - und kann es nicht sein. (...) Der gut informierte Bürger ist weder ein 'Rechter' noch ein 'Linker', weder ein 'Konservativer' noch ein 'Fortschrittlicher' - noch ein Dritter in der 'Mitte', noch ein 'Kritischer' an den Rändern. Vielmehr variiert der Typus des 'gut informierten Bürgers' hinsichtlich dieser oder anderer Wert- oder Positionspräferenzen in der gesamten Bandbreite des Wert- und PositionsSpektrums, das einer pluralistischen Gesellschaft ihre typische Gestalt gibt." ${ }^{73}$

daß die Befragung erst im Mai 1987, also ein Jahr nach dem Reaktorunfall, stattfand. Die höchsten Glaubwürdigkeitswerte (operationalisiert mit der Frage nach der Verläßlichkeit ihrer Informationen) wiesen Regierung, Opposition und Kernforschungszentren auf; die höchste Vertrauenswürdigkeit (operationalisiert mit der Frage nach dem Interesse an öffentlichen Belangen vs. Eigeninteresse) Regierung, Opposition und Anti-Atomkraft-Gruppen; die höchste Kompetenz wurde Forschungszentren und Industrie zugesprochen. Journalisten wurden weder als besonders vertrauenswürdig noch als kompetent eingestuft, obwohl die Medien, insbesondere das Fernsehen, die wichtigsten Informationsquellen darstellen. (Vgl. Helmut Lukesch / KarlHeinz Kischkel / Wolfgang Nöldner: Zur Beurteilung von Informationsquellen und Medien im Zusammenhang mit dem Reaktorunfall von Tschernobyl, in: Publizistik 32/1987, S. 154-158.) In der Tendenz übereinstimmende Einschätzungen von Kompetenz und Vertrauenswürdigkeit werden auch im Zusammenhang mit der Berichterstattung über Umweltrisiken im Bereich Chemie berichtet. (Vgl. David B. McCallum / Sharon Lee Hammond / Vincent T. Covello: Communicating about Environmental Risks: How the Public Uses and Perceives Information Sources, in: Health Education Quarterly 18/1991, S. 349-361.)

Vgl. Peters (1992), S. 336.

Wagner (1988), S. 29f. [Hervorhebungen im Original gesperrt gedruckt.] 
Entsprechend muß das Informationsangebot so beschaffen sein, daß sich jeder Bürger Orientierung verschaffen und "zu je aktuellen Fragen sozusagen sein 'eigenes Lager' ausfindig" machen sowie die Nähe zu anderen "sozialen Standorten" ausloten kann. ${ }^{74}$ Diese Chance ist ihm genommen, wenn Vertrauensinformationen unvollständig, verkürzt oder verzerrt dargeboten werden. Aber nicht nur die Unterschlagung zentraler Merkmale, die zur Stärkung der Glaubwürdigkeit eines mißliebigen Ausgangspartners beitragen können, kennzeichnet die publizistische Strategie. Umgekehrt ist es natürlich genauso irreführend, Partner, die die Sichtweise des Publizisten stützen, besonders glaubwürdig erscheinen zu lassen, indem nicht zutreffende Merkmale vermittelt oder vorhandene überakzentuiert werden. Man muß dabei nicht so plump vorgehen wie die Bild-Zeitung, die "Deutschlands besten Kriminologen" zur Untermauerung der These des Mordes an Uwe Barschel zu Wort kommen ließ. ${ }^{75}$ Bereits die Kennzeichnung einer Position mit dem Etikett "Experte" kann eine solche Akzentuierung darstellen, insbesondere, wenn die Begründung, was genau diesen Sprecher zum Experten macht, unterbleibt.

Für die Einschätzung der Glaubwürdigkeit werden, wie der Forschungsüberblick zur quellenorientierten Glaubwürdigkeitsforschung verdeutlicht, neben den genannten Hinweisen auf Herkunft, Kompetenz, Funktion und Soziale Billigung noch eine Reihe weiterer Kriterien herangezogen, die publizistischer Beeinflussung unterliegen können. Die 'Vertrauenswürdigkeit' eines Ausgangspartners kann erhärtet oder zerstört werden durch Verweise auf Rechtschaffenheit oder Unehrlichkeit oder dadurch, daß die Lauterkeit seiner Motive betont oder in Frage gestellt wird. Neben solch personenbezogenen

$74 \quad$ Vgl. ebd. S. 30.

75

Vgl. Bild vom 16.10.1987, S. 1. Die gleiche Strategie verwendete Bild laut Kepplinger/Hartung bei der Berichterstattung über einen Störfall bei der Firma Hoechst: In verschiedenen Beiträgen ist vom "größten Giftexperten Deutschlands", "Deutschlands berühmtestem GiftForscher" und "Deutschlands bestem Giftforscher" die Rede. (Vgl. Hans Mathias Kepplinger / Uwe Hartung: Störfall-Fieber. Wie ein Unfall zum Schlüsselereignis einer Unfallserie wird, Freiburg/München 1995, S. 94f.) 
Zuschreibungen kann auch die Auseinandersetzung mit der Aussage des Ausgangspartners ins Gewicht fallen: Durch Hinweise auf deren Schlüssigkeit oder Widersprüchlichkeit können Zweifel gesät oder ausgeräumt werden. 'Dynamik' und Sprechverhalten des Ausgangspartners können den Rezipienten durch Schilderungen nahegebracht werden; durch die Beschreibung von Verhaltensweisen oder Urteile über die Gemütsverfassung können Stabilität und Aufrichtigkeit ebenso suggeriert werden wie Unsicherheit und Nervosität. Schließlich können Urteile und Wertungen über Charaktereigenschaften oder Erscheinungsbild, die geeignet sind, den Sprecher sympathisch oder unsympathisch darzustellen, das Bild komplettieren.

Zuschreibungen in allen Dimensionen können darüberhinaus "begründet" oder "unbegründet" erfolgen. Als "Begründungen" sind nachvollziehbare, gegebenenfalls nachprüfbare Hinweise aufzufassen, die Belege oder Beweise für das Gesagte darstellen. Solche Begründungen haben zugleich die Funktion, die Glaubwürdigkeit desjenigen, der das Urteil abgibt - sei es der Publizist selbst oder ein Dritter - zu erhärten.

\subsection{Die Glaubwürdigkeit anonymer Quellen}

Die bisherigen Ausführungen konzentrierten sich vornehmlich auf die Manipulationsmöglichkeiten bei der Vorstellung namentlich genannter Ausgangspartner. Wie an der oben zitierten Spiegel-Passage deutlich wurde, können aber auch anonyme Quellen eine Rolle im Zeitgespräch spielen. 'Anonym' bedeutet hier zunächst, daß keine Namensnennung vorliegt; darüberhinaus gibt es unterschiedliche Grade der Anonymität. So ist der Informationsverlust für den Leser gering, wenn er zwar keinen Namen erfährt, jedoch Anhaltspunkte bezüglich sachlicher Herkunft und Funktion der Quelle vorliegen (z.B.: "ein Polizeisprecher erklärte..."). ${ }^{76}$ Je mehr solcher Hinweise fehlen, desto mehr

76 Das Problem ist journalistischen Praktikern durchaus bewußt: So zitiert Zschunke (1994: 126) die Forderung des AP-Handbuchs, Personen "wenn immer das möglich ist, mit vollem 
wird ihm die Orientierung erschwert: Kann er eine Mitteilung "aus Regierungskreisen" noch zumindest grob verorten, lassen Markierungen wie "es heißt" oder "es ist zu hören" keine Rückschlüsse auf die Herkunft mehr zu. Allerdings sind solche Anonymisierungen nicht automatisch schon Hinweise auf publizistische Manipulationsversuche; in Einzelfällen kann es durchaus gerechtfertigt sein, auf diese Weise Informanten zu schützen. ${ }^{77}$

Für den Rezipienten bleibt die Konsequenz, daß er sich in Fällen mangelhafter Quellentransparenz kein eigenes Bild über die Glaubwürdigkeit solcher Quellen machen kann. Vielmehr muß er sich auf den Vermittler verlassen, der anonyme Ausgangspartner nicht selten mit Glaubwürdigkeitsmerkmalen ausstattet, die keiner Prüfung zugänglich sind: Die bekannten "gutunterrichteten Kreise" verweisen ebenso wie nicht namentlich genannte "Insider" oder "Experten" auf Kompetenz, "zuverlässige Informanten" legen Vertrauenswürdigkeit nahe.

Daß solche Kennzeichnungen ihre Wirkung nicht verfehlen, belegt eine Studie von Adams, der seinen Versuchspersonen eine Liste von 20 anonymen Quellen vorlegte und deren Glaubwürdigkeit beurteilen ließ. ${ }^{78}$ Bei diesem

Namen zu nennen" und erläutert: "Dieser Grundsatz aus dem stark personalisierten amerikanischen Journalismus wird in Deutschland weniger strikt praktiziert - da beispielsweise der Name eines Polizei- oder Feuerwehrsprechers in der Regel keinerlei Informationswert besitzt, gilt hier die Namensnennung als entbehrlich..."

77 Zur Rechtfertigung merkt Weischenberg (1990: 217) an: "Nicht nachprüfbare (ungenaue, fehlende) Quellenangaben sind grundsätzlich problematisch. Dies gilt auch für die von Nachrichtenmedien bei anonym präsentierten Informationen häufig bemühten 'informierten Kreise' - eine Krücke, auf die freilich viele Journalisten nicht verzichten wollen oder können. Denn manchmal - z.B. bei Berichten aus dem kriminellen Milieu - ist es notwendig, die Informanten durch eine allgemein gehaltene Quellenangabe zu schützen." Und Zschunke(1994: 121) führt aus: "Meldungen 'aus Regierungskreisen' sind in der Auslandsberichterstattung oft häufiger anzutreffen als zur Innenpolitik. Da die offiziellen Pressesprecher der Regierungen in den einzelnen Staaten eine sehr vorsichtige Informationspolitik betreiben, ist es für die Korrespondenten von entscheidender Bedeutung, einen guten Kontakt zu hohen Beamten zu finden, die sie mit Informationen versorgen. Diese sind aber meistens nur dann zur Zusammenarbeit bereit, wenn sie sicher sein können, daß ihre Anonymität gewahrt bleibt." lism Quarterly 39/1962, S. 79-82. Die beiden Quellen, die hier die höchsten Glaubwürdigkeits- 
Vergleich schnitten Quellen, die auf offizielle oder amtliche Herkunft verwiesen (z.B. "official reports", "official sources", "a government spokesman"), am besten ab. ${ }^{79}$ Ähnlich gute Werte erreichten auch die "Experten". Mittlere Positionen belegten "Gewährsmänner" ("authorities") und "gut informierte Stellen". Am Ende der Skala waren Hinweise angesiedelt, die keine Anhaltspunkte zur Orientierung lieferten ("it was learned", "an announcement", "indications" - wobei "trustworthy indications" noch etwas besser eingestuft wurden). Adams folgert: "We might speculate that readers look for something at least a little more definite in their news stories than the sort of source which could represent no more than the reporter and his imagination." ${ }^{80}$

Zumindest im direkten Vergleich bestätigt sich also die Annahme, daß ein sinkender Anteil an 'Vertrauensinformationen' zu einer Abnahme der Glaubwürdigkeit der Quellen führt. Eine Folgestudie sollte den Nachweis erbringen, daß solche Rangfolgen sich nicht nur im abstrakten Vergleich, sondern auch im Kontext einer Nachricht ergeben. Diese Hypothese konnte jedoch nicht bestätigt werden.$^{81}$ Für die nicht konsistenten Ergebnisse verantwortlich ist möglicherweise zum Teil die Kombination der gewählten Quellen ("official reports", "authorities" und "trustworthy indications") mit den Themen. So schneiden wohl die "offiziellen Meldungen" in Berichten über Rußland und

werte erzielten - "die US-Regierung" und "die Regierung" - sind nach zeitungswissenschaftlicher Auffassung allerdings nicht anonym, sondern es handelt sich dabei um genannte Institutionen.

79 Vgl. ebd. S. 80ff. Einzige Ausnahme bildeten "official circles"; offenbar löste die Bezeichnung "Kreise" ein Mißtrauen aus, das auch durch die Beigabe des Adjektivs nicht beschwichtigt werden konnte.

80 Ebd. S. 81. [Hervorhebung im Original.] Daß sich hinter solch mangelhaft gekennzeichneten Quellen der Vermittler selbst verbirgt, ist nur eine Möglichkeit. Schneider benennt weitere: "Welcher Medienkonsument wittert hinter den 'gut informierten Kreisen' einen geltungssüchtigen Regierungsrat oder den Taxifahrer des Korrespondenten, hinter 'politischen Beobachtern' den Barmann eines Honkonger Hotels?" (Wolf Schneider (Hrsg.): Unsere tägliche Desinformation. Wie die Massenmedien uns in die Irre führen, Hamburg 1984, S. 46.)

81

Vgl. John B. Adams: Unnamed Sources and the News: A Follow-Up Study, in: Journalism Quarterly 41/1964, S. 262-264. 
China deshalb so schlecht ab, weil sie mit den dortigen Regierungen in Verbindung gebracht werden und daher als parteilich gelten. Adams bietet eine weitere Interpretationsmöglichkeit:

"Ex post facto, it can be seen that the subjects' frames of reference were important. Most subjects' reasons for answering as they did were based on prior knowledge (or misinformation in many cases) or on their idea of what was 'logical', presumably in the light of their perception of the facts of the stories." 82

Es scheint also wahrscheinlich, daß vage Quellenangaben dazu führen, daß Rezipienten zur Beurteilung der Glaubwürdigkeit sich eher den Textinhalten zuwenden. $^{83}$ Für diese Einschätzung spricht auch, daß sich zwischen quellenund mitteilungsorientierten Rezipienten keine Unterschiede ergaben; auch quellenorientierte Personen ließen sich von den anonymen Quellen nicht beeinflussen.

Eine Wiederholung der Untersuchung von Adams knapp 20 Jahre später erbringt geringere Glaubwürdigkeitswerte für anonyme Quellen aus Kreisen der Regierung, die als generelles Mißtrauen der Regierung gegenüber in der Folge von 'Watergate' und Vietnam-Krieg interpretiert werden. Demgegenüber gewinnen anonyme Experten an Glaubwürdigkeit, während unbestimmbare Quellen am Ende der Skala angesiedelt bleiben:

"Sources lacking a concrete referent remained less acceptable sources of information than did sources having some referent to a person, document or institution. (...) Like their 1960 counterparts, 1979 respondents were suspicious of this sort of reportorial judgment and conclusion-drawing. Perhaps they view this interpretation as inconsistent with their expectations of good journalistic practice, and they simply draw the line at such vagueness." 84

$82 \quad$ Adams (1964), S. 264.

83 Ob sie dies auch tun, wenn sie nicht durch eine Experimentalsituation zu einer unmittelbaren Stellungnahme gezwungen sind, muß der Spekulation überlassen bleiben.

84

Daniel Riffe: Relative Credibility Revisited: How 18 Unnamed Sources Are Rated, in: Journalism Quarterly 57/1980, S. 618-623, S. 621. 
Auch die Ombudsfrau der 'Washington Post', Geneva Overholser, bemängelt in ihrer ersten Kolumne den nachlässigen Umgang mit Quellen:

"Schrecklich ist, wie oft das Blatt Leute etwas sagen läßt, die nicht beim Namen genannt werden. Allerorten werden anonyme Quellen verwendet - und das führt gerade hier in Washington zu Konsequenzen, die nicht zu rechtfertigen sind. Wir müssen uns zumindest klarmachen, wie sehr das unserer Glaubwürdigkeit schadet." 85

Zschunke gibt ebenfalls zu bedenken, daß bei einer Häufung von anonymen Kennzeichnungen auch die Glaubwürdigkeit des vermittelnden Mediums auf dem Spiel steht:

"Es liegt im Interesse des Journalisten, einen verläßlichen Informanten vor unliebsamen Konsequenzen zu schützen. Doch um der eigenen Glaubwürdigkeit willen sollten anonyme Quellenangaben so selten wie möglich benutzt werden und zwar nur dann,

* wenn es sich um eine berichtenswerte Tatsache und nicht um Meinungen handelt,

* wenn der Informant auf Wahrung der Anonymität besteht,

* wenn die mitgeteilte Information nicht auf anderem Weg beschafft werden kann." 86

Die Eingrenzung, bei "berichtenswerten Tatsachen" im Gegensatz zu Meinungsäußerungen auf die Nennung der Quelle zu verzichten, ist allerdings nicht ohne weiteres nachvollziehbar, da es "eine von allen Subjektivismen gereinigte, nackte Faktensicht kaum gibt". ${ }^{87}$

Für die Orientierung der Rezipienten noch problematischer ist in diesem Zusammenhang, wenn Sachverhalte ganz ohne Quellenkennzeichnung als "Tatsachen" dargestellt werden, wie sich an einem Experiment von Smith zeigen läßt: Er konstruierte fiktive Nachrichten und variierte deren Etikettie-

85 Zitiert nach: Stephan Ruß-Mohl: Die Nebenchefin, in: MediumMagazin 10/1995, S. 7072, S. 71

$86 \quad$ Zschunke (1994), S. 127.

87 Schröter (1995) S. 47 
rung ('fact' oder 'rumor'). Eine Kennzeichnung mit dem Label "fact" führte dabei immer zur höchsten Zustimmung, unabhängig von der Einstellung der Befragten zum Thema, in fünf von sechs Fällen führte die Quellenangabe "Gerücht" zu einer schlechteren Einstufung als gar keine Quellenkennzeichnung. ${ }^{88}$ Der Unterschied war allerdings nicht allzu ausgeprägt. Das Label "Gerücht" führte nicht automatisch dazu, daß der Inhalt nicht geglaubt wurde; wie bei der "no label"-Bedingung hing die Zustimmung zur Aussage dann in erster Linie davon ab, wie plausibel das Statement war. ${ }^{89}$ Smith zieht die Schlußfolgerung:

\footnotetext{
"In more realistic situations, where the 'message' is artfully tuned to deep motives and where argument has become habitual, a 'truth' or 'fact' label might considerably increase acceptance. For example, a trusted newspaper, an accepted leader, a favourite radio commentator might have noticeable effects on a particular public by asserting or implying that some unauthenticated statement was really true." 90
}

Dieser Befund wird untermauert durch weitere, die besagen, daß eine Mitteilung, bei der ein Kommunikator gänzlich ungenannt bleibt, größere Einstellungsänderungen erzielen kann als dieselbe Mitteilung von einem Kommunikator, der über geringe Glaubwürdigkeit verfügt. ${ }^{91}$

88 Vgl. George Horsley Smith: Beliefs in Statements Labeled Fact and Rumor, in: Journal of Abnormal and Social Psychology 42/1947, S. 80-90, S. 84f.

$89 \quad$ Vgl. ebd. S. 86f.

$90 \quad$ Ebd. S. 88

91 Vgl. Frederic A. Powell / Gerald R. Miller: Social Approval and Disapproval in AnxietyArousing Communications, in: Speech Monographs 34/1967, S. 152-159 und Greenberg / Miller (1966). Johnson und Scileppi (1969: 35) weisen, ähnlich wie Smith, darauf hin, daß dies nur gilt, wenn der Kommunikationsinhalt in sich plausibel ist. Siehe ferner auch Homer H. Johnson / Richard R. Izzett: The Effects of Source Identification on Attitude Change as a Function of the Type of Communication, in Journal of Social Psychology 86/1972, S. 81-87. 


\subsection{Zwischenbilanz}

Die Untersuchung der Glaubwürdigkeit verschiedener Akteure in Massenmedien verlangt ein Kommunikationsmodell, das berücksichtigt, daß jede Aussage auf seinen Urheber zurückgeführt werden können muß. Die zeitungswissenschaftliche Massenkommunikationstheorie legt ein solches Modell vor. Zentrales Merkmal dieses Modells ist die Trennung von Mitteilungs- und Vermittlungsprozeß. Entsprechend ist es gekennzeichnet durch eine zweipaarige Rollenstruktur: die Kommunikationsrollen werden eingenommen von Ausgangspartnern und Zielpartnern, die Vermittlungsrollen haben autonome Vermittlungssysteme und Rezipienten inne. Den Vermittlungssystemen obliegt es - neben den technischen Vermittlungsleistungen der Signalproduktion und Distribution - redaktionelle Leistungen zu erbringen, um die Aufrechterhaltung sozialer Kommunikation zu gewährleisten.

Bei der Erfüllung dieser redaktionellen Aufgaben (Selektion von Ausgangspartnern und deren Aussagen sowie mediengerechte Transformation der Aussagen) können idealtypisch zwei Vermittlungsstrategien unterschieden werden, die die Möglichkeit der Orientierung für den Rezipienten maßgeblich beeinflussen: Journalistische Vermittlung zeichnet sich aus durch Unparteilichkeit und Objektivität, die sich manifestieren in der Wiedergabe aller relevanten Gesprächspositionen bei mitteilungsadäquater Transformation der Aussagen sowie in der Abwesenheit von Wertungen seitens des Vermittlers. Publizistische Vermittlung ist gekennzeichnet durch Parteilichkeit, was dazu führt, daß der vermittelte Partnerkosmos unvollständig bleibt, da nicht genehme Standpunkte unterschlagen werden, daß Manipulationen bei der Selektion und Transformation der Aussagen erfolgen oder das Postulat der Trennung von Nachricht und Kommentar nicht gewahrt wird.

Fragt man nach der Glaubwürdigkeit der an der Kommunikation beteiligten Akteure, so ist zu unterscheiden zwischen der Glaubwürdigkeit der Vermittlungsinstanzen (der Medien und ihrer Vermittler), der Glaubwürdigkeit 
der zu Wort kommenden Ausgangspartner und der Glaubwürdigkeit von Kommunikations-Objekten, über die gesprochen wird. Im Rahmen eines Beitrags können Ausgangspartner dabei gleichzeitig zu Objekten der Kommunikation werden. Hinsichtlich der zentralen Faktoren, die als Glaubwürdigkeitsmerkmale anzusehen sind, gibt es Übereinstimmungen zwischen Vermittlungsinstanzen, Ausgangspartnern und Kommunikationsobjekten, jedoch äußern sich diese in unterschiedlichen Ausprägungen.

Kompetenz äußert sich bei den Medien in erster Linie als Vermittlungskompetenz. Vermittlungskompetenz meint den sachgerechten Umgang mit Quellen und deren Mitteilungen hinsichtlich ihrer Selektion und Transformation sowie eine korrekte Identifikation der Ausgangspartner, die die Orientierung der Rezipienten gewährleistet. Vertrauenswürdigkeit manifestiert sich, was die Medien anbelangt, in der Erfüllung der Forderung nach Fairneß, Unparteilichkeit und Objektivität. Die Dynamik verlagert sich bei den Medien auf formale Elemente der Präsentation. Ungeklärt ist, wie sich Aspekte der Ähnlichkeit und der Sympathie auf die Glaubwürdigkeit der Medien auswirken.

Die Glaubwürdigkeit von Ausgangspartnern und Kommunikations-Objekten in der Massenkommunikation gründet sich wie in der direkten Kommunikation auf die Dimensionen 'Kompetenz', 'Vertrauenswürdigkeit', 'Dynamik' und 'Sprechverhalten', 'Ähnlichkeit', 'Soziale Billigung' und 'Sympathie'. Welche dieser Dimensionen überhaupt zur Sprache kommen und welche Aspekte innerhalb der einzelnen Dimensionen thematisiert werden, ist abhängig vom Vermittler. Im Fall der Ausgangspartner ist er der einzige, der Kriterien zur Einordnung bereitstellt. Die Glaubwürdigkeit von Personen, die lediglich als Objekte der Berichterstattung auftreten, steht zusätzlich in Abhängigkeit von den Urteilen, die gesellschaftliche Ausgangspartner über sie abgeben, wobei letztere wiederum von den Vermittlern ausgewählt werden.

Damit kommt den Vermittlern bei der Schaffung oder Zerstörung der Glaubwürdigkeit gesellschaftlicher Akteure eine zentrale Rolle zu. Ihre Aufgabe ist es, die relevanten 'Vertrauens-Informationen' vollständig und 
unverzerrt bereitzustellen. Dies deckt sich mit den Anforderungen, die an eine 'journalistische' Vermittlungsstrategie zu stellen sind. Dabei können die 'Vertrauens-Informationen' durchaus den Charakter von "Mißtrauens-Informationen" annehmen, ohne daß der Vermittler seine Position als 'Journalist' verläßt. Nicht, ob die dargebotenen Informationen "positiv" oder "negativ" sind, kennzeichnet die Trennung von journalistischer und publizistischer Vermittlungsstrategie, sondern ausschließlich die An- oder Abwesenheit von Parteilichkeit. Entsprechend besteht die publizistische Einflußnahme auf die Wahrnehmung von Glaubwürdigkeit darin, daß - in einzelnen oder allen Dimensionen - Merkmale verschwiegen oder nicht zutreffende Merkmale vermittelt werden, oder darin, daß vorhandene Merkmale über- oder untertrieben werden. 


\section{Der politische Skandal und die Rolle der Medien}

\subsection{Definition des 'politischen Skandals'}

"Es kommt plötzlich etwas heraus, ruft öffentliche Empörung (und Lust an der Empörung) hervor, wird für die Normen öffentlichen Verhaltens in negativer Weise als bezeichnend empfunden und hat politische Bedingungen und Folgen". ${ }^{1}$ Diese Definition, die übereinstimmend von verschiedenen Autoren präsentiert wird, ${ }^{2}$ umfaßt bereits drei zentrale Elemente des Skandals: Es muß

Wolfgang Schuller: Frevel, Raub, Bestechung - Volksversammlung und Senat. Skandale und Öffentlichkeit in der griechischen und römischen Welt, in: Rolf Ebbinghausen / Sighard Neckel (Hg.): Anatomie des politischen Skandals, Frankfurt a.M. 1989, S. 83-103, S. 83.

2 Diese Festlegung deckt sich mit der von Schütze, der den Skandal als "öffentliche Erregung über ein allgemein empfundenes Ärgernis" charakterisiert. (Vgl. Christian Schütze: Skandal. Eine Psychologie des Unerhörten, Bern/München ${ }^{2} 1985$, S. 8.) Etwas ausführlicher aber sinngemäß übereinstimmend siehe auch: Karl Otto Hondrich: Skandale als gesellschaftliche Lernmechanismen, in: Julius H. Schoeps (Hrsg.): Der politische Skandal, Stuttgart u.a. 1992, S. 175-189, S. 179: "Unter einem Skandal verstehe ich einen sozialen Prozeß, der durch drei Merkmale charakterisiert ist: erstens durch moralische Verfehlungen, ganz gleich, ob diese tatsächlich begangen werden oder hochgestellten Personen oder Institutionen nur unterstellt werden. Zweitens durch die Enthüllung einer Verfehlung, die zuvor unbekannt war oder verborgen gehalten wurde. Und drittens durch eine weithin geteilte Empörung, die sich aufgrund dieser Enthüllung einstellt. Erst wenn alle drei Charakteristika zusammenkommen, kann es einen Skandal als gesellschaftliches Phänomen geben." [Hervorhebung im Original.] Siehe ferner: Carl Böhret / Werner Jann: Verwaltungsskandale, in: Aus Politik und Zeitgeschichte (Beilage zu 'Das Parlament') B 27/1982, S. 35-52, S. 36; Gerhard Pippig: Verwaltungsskandale. Zur Korruption in der öffentlichen Verwaltung, in: Aus Politik und Zeitgeschichte (Beilage zu 'Das Parlament') B7/1990, S. 11-20, S. 11; Hans-Joachim Winkler: Über die Bedeutung von Skandalen für die politische Bildung, in: Aus Politik und Zeitgeschichte (Beilage zu 
eine Normverletzung vorliegen, diese Normverletzung muß Empörung hervorrufen und zwar keine singuläre, vereinzelte Empörung sondern "öffentliche", was bedingt, daß ein größerer Personenkreis Kenntnis von dem "Ärgernis" erlangt. Das was "herauskommt", die Normverletzung also, ist dabei ausschließlich auf menschliches Tun zurückzuführen:

"Wer politische und gesellschaftliche Ereignisse mit Naturereignissen vergleicht, erweckt den Eindruck einer naturhaften Zwangsläufigkeit. (...) Aus soziologischer Sicht unterschlägt und mystifiziert eine derartige Betrachtungsweise die entscheidende Tatsache, daß ein 'Skandal' ... eine von Menschen durch ihr aktives und passives Handeln erzeugte Folge von Handlungen ist, die keinerlei 'Natur'gesetzen gehorchen."

In den Definitionen ist ferner angedeutet, daß ein Skandal nur denkbar ist, wenn Kommunikation stattfindet. Die beteiligten Akteure bilden dabei eine Skandal-Triade. Zum Skandal gehören:

"...der Skandalierte (der einer Verfehlung von öffentlichem Interesse öffentlich bezichtigt wird), der Skandalierer (einer, der diese Verfehlung öffentlich denunziert) sowie ein, oder besser: mehrere Dritte, denen über das, was zum Skandal geworden ist, berichtet wird und die daraufhin eine wie auch immer geartete Reaktion zeigen." 4

Eine Möglichkeit, die definitionsgemäß notwendige Empörung kundzutun, besteht darin, die Normverletzung explizit mit der Bezeichnung 'Skandal' (oder verwandten Bezeichnungen wie 'Affäre' oder 'Fall') zu versehen. Diese Etikettierung kann als weiteres Definitionsmerkmal betrachtet werden:

"Und der Skandal soll nun eben als jener Vorgang gelten, in dem es einem Akteur (dem

'Das Parlament') 27/1968, S. 3-14, S. 5.

3 Dirk Käsler et al.: Der politische Skandal. Zur symbolischen und dramaturgischen Qualität von Politik, Opladen 1991, S. 11.

4 Sighard Neckel: Das Stellhölzchen der Macht. Zur Soziologie des politischen Skandals, in: Leviathan 14/1986, S. 581-605, S. 585. [Eigene Hervorhebung; U.N.] 
Skandalierer) gelingt, durch die Thematisierung eines Ereignisses oder eines Sachverhalts im Zusammenhang mit einem Akteur (dem Skandalierten) die Erwartungen eines anderen Akteurs (des Skandalpublikums) zu irritieren. Der Begriff 'Skandal' bezeichnet demnach nicht die Verletzung einer sozial gültigen Norm, sondern die akzeptierte Etikettierung eines Ereignisses oder Sachverhalts als nicht normenkonform. Kurz: Ein Sachverhalt wird dadurch zum Skandal, daß er bekannt gemacht und erfolgreich als Skandal definiert worden ist." ${ }^{5}$

Ein Skandal resultiert also aus dem "selektiven Einsatz von Definitionsmacht", das heißt, ein Zustand muß glaubhaft als 'skandalös' präsentiert werden. Nicht das Ereignis an sich ist schon als Skandal anzusehen, vielmehr wird es von interessierter Seite als solcher präsentiert ${ }^{6}$ :

"Ein Skandal ist Ansichtssache - jedenfalls in segmentierten, in eine Vielzahl von Auffassungen, Ideologien und Glaubensrichtungen zerfallenen Gesellschaften wie der unseren. Das bedeutet, daß kaum noch irgendein Vorfall oder Sachverhalt unisono zum Skandal erklärt bzw. als ein solcher wahrgenommen wird. Zugleich heißt das auch: Es ereignet sich kaum etwas mit dem Anschein politischer Virulenz, das nicht von irgend jemandem als Skandal empfunden und 'irgendwie' auch als ein solcher lanciert würde." ${ }^{7}$

Gelegentlich wird versucht, hinsichtlich der Normverletzung oder der beteiligten Akteure zu präzisieren:

"Skandale ragen im Vergleich zu Straftaten, die jedermann begeht, als besonders hochkarätige

$5 \quad$ Ronald Hitzler: Skandal ist Ansichtssache. Zur Inszenierungslogik ritueller Spektakel in der Politik, in: Rolf Ebbinghausen / Sighard Neckel (Hg.): Anatomie des politischen Skandals, Frankfurt a.M. 1989. S. 334-354, S. 334. [Hervorhebungen im Original.]

6 Vgl. Ronald Hitzler: Skandal: Karrierebremse oder Karrierevehikel? Inszenierungsprobleme Bonner Parlamentarier, in: Sozialwissenschaftliche Informationen 1/1987, S. 22-27, S. 24. Neckel und Szasz sprechen in diesem Zusammenhang von einem "Definitionskampf", der jedem Skandal vorausgeht. (Vgl. Sieghard Neckel: Machen Skandale apathisch? In: Ebbinghausen / Neckel (1989), S. 234-257, S. 243f.; Andrew Szasz: The Process and Significance of Political Scandals: A Comparison of Watergate and the "Sewergate" Episode at the Environmental Protection Agency, in: Social Problems 33/1986, S. 202-217, S. 212.)

Neckel (1989), S. 24. [Hervorhebung im Original.] 
Verstöße gegen Recht und Moral heraus: gelegentlich durch die Schwere des Verstoßes, immer aber aufgrund der hohen Stellung der Betroffenen, von denen kraft Amtes und sozialen Standes eine vorbildliche Beachtung sozialer Normen und Verantwortung erwartet wird. Mit der herausgehobenen Stellung der unmittelbar Betroffenen hängt das zweite charakteristische Merkmal des Skandals zusammen: seine Öffentlichkeit. Normverstöße und Öffentlichkeit begründen zu Recht eine zwiespältige Einstellung gegenüber Skandalen; denn beides, die Definition eines Handelns als Unrecht und seine Veröffentlichung, kann von interessierter Seite lanciert werden." 8

$\mathrm{Da}$ die Art des Normverstoßes oder das Gewicht, das ihm zukommt, ausschlaggebend für das Zustandekommen eines Skandals ist, wird in dieser ebenso wie in den vorangegangenen Aussagen relativiert. Bedeutsamer als die Schwere der Verfehlung ist unter Umständen das Ausmaß der Empörung, die hervorgerufen wird. Insbesondere bei Personen in übergeordneter Stellung kann bereits ein verhältnismäßig geringfügiger Normverstoß einen Skandal auslösen, sofern es den Skandalierern gelingt, Entrüstung zu produzieren und aufrechtzuerhalten.

Als 'politisch' soll ein Skandal dann gelten,

"wenn die Akteure des politisch-administrativen Systems unmittelbar und auslösend in ihn verwickelt sind, wenn das normative Inventar der Politik durch ihn berührt wird und der Skandal die Form jener Handlungsfiguration annimmt, die in der politischen Soziologie als Konflikt bezeichnet wird." ${ }^{9}$

Diese Definition soll für die vorliegende Arbeit Gültigkeit haben. Akteure (das heißt: Skandalierer oder Skandalierte) politischer Skandale können also politische Mandatsträger sein, Minister und ihre Untergebenen ebenso wie Angehörige von Behörden und Administration, Parteien oder einzelne Parteimitglieder sowie Mitarbeiter in staatlichen Unternehmen; mithin politische 
Institutionen als Ganzes sowie Angehörige sämtlicher Hierarchieebenen. ${ }^{10}$

\subsection{Die Rolle der Medien}

Das Herstellen von Öffentlichkeit geschieht in modernen Gesellschaften mittels Massenmedien, was vereinzelt ausdrücklich betont wird. Die Rolle der Medien wird dabei als weitgehend passiv eingestuft: Entweder sie "transportieren" Skandale $^{11}$ oder sie decken vorhandene Skandale auf. ${ }^{12}$ Demgegenüber postulieren Kepplinger und Hartung eine aktive Rolle der Massenmedien:

"Zum Skandal werden im Medienzeitalter Mißstände nur dann, wenn die Massenmedien sie als Skandal darstellen. Deshalb ist es falsch zu sagen: Die Massenmedien decken Skandale auf und berichten über sie. Vielmehr charakterisieren sie Mißstände als empörend - und machen sie dadurch zu einem Skandal, zu einem öffentlichen Ärgernis. Ob diese Charakterisierung angemessen und richtig ist, steht auf einem anderen Blatt." ${ }^{13}$

10 Abweichend von dieser Auffassung grenzen verschiedene Autoren 'Verwaltungsskandale' von politischen Skandalen ab: "Verwaltungsskandale sind dann gegeben, wenn die Normverletzung nicht überwiegend personalisiert ist, sondern mit der Institution 'öffentliche Verwaltung' direkt verbunden ist (also vorrangig eine institutionelle 'Schuld' vorliegt)." [Böhret / Jann (1982), S. 36. Vgl. auch Pippig (1990).]

11 Vgl. Käsler et al. (1991), S. 13; Siegfried Preisler: Ganz normale menschliche Reaktionen. Skandalverarbeitung im Spannungsfeld politischer Erfahrungen, Werte und Einstellungen, in: Helmut Moser (Hrsg.): L' Eclat c'est moi. Zur Faszination unserer Skandale, Weinheim 1989, S. 98-117, S. 100.

12 Vgl. Ulrich von Alemann: Politische Moral und politische Kultur in der Bundesrepublik - Vergiften oder reinigen Skandale die Politik? in: Gewerkschaftliche Monatshefte 5/1985, S. 258-269, S. 264.

13 Hans Mathias Kepplinger / Uwe Hartung: Am Pranger. Eine Fallstudie zur Rationalität öffentlicher Kommunikation, München 1993, S. 5. Vgl. auch Hans Mathias Kepplinger et al.: Am Pranger: Der Fall Späth und der Fall Stolpe, in: Wolfgang Donsbach et al.: Beziehungsspiele - Medien und Politik in der öffentlichen Diskussion. Fallstudien und Analysen, Gütersloh 1993, S. 159-220, S. 159; Hans Mathias Kepplinger / Peter Eps / Dirk Augustin: Skandal im Wahlbezirk. Der Einfluß der Presse auf die Wahl des Münchner Oberbürgermeisters 1993, in: Publizistik 40/1995, S. 305-326, S. 306. 
Dagegen konstatiert Neckel: "Die Medien sind heute zwar insofern meist die Urheber des Skandals, als sie für die Visibilität der Verfehlungen sorgen; hinsichtlich der öffentlichen Reaktionen über das Skandalgeschehen können ihnen aber allenfalls Verstärkerfunktionen zukommen."14

Tatsächlich läßt sich die Rolle der Medien nicht a priori festlegen. Vielmehr hängt sie davon ab, welche Vermittlungsstrategie jeweils gewählt wird. Zwar können Medien die Rolle des Skandalierers einnehmen - der Spiegel als "unumstritten zentrale Skandalisierungsinstitution in der Bundesrepublik"15 demonstriert dies nahezu wöchentlich - jedoch kann nicht allein aus der Tatsache, daß sie Skandale öffentlich machen, abgeleitet werden, daß sie "Skandale machen". Auch Vermittler, die sich in erster Linie als 'Journalisten' verstehen, haben die Aufgabe, über Skandale zu berichten. Nicht daß sie es tun ist entscheidend für die Bestimmung ihrer Rolle, sondern wie sie es tun.

Ein 'journalistischer' Umgang mit Skandalen ist dadurch gekennzeichnet, daß ausnahmslos alle gesellschaftlichen Positionen, die zu den Vorgängen auszumachen sind, mitteilungsadäquat vermittelt werden, ohne daß der Vermittler Partei ergreift. ${ }^{16}$ Er schaltet sich also weder in den KommunikationsProzeß ein, noch übernimmt er eine Rolle in der Skandal-Triade, und er gewährt auch keinem der beteiligten Skandal-Akteure einen Vorteil, indem er dessen Gesprächsbeitrag bevorzugt behandelt. Diese neutrale Rolle findet auch bei Hitzler Berücksichtigung, wenn er der Triade von Skandalisierer, Skandalisiertem und Skandalrezipient den Skandalvermittler hinzufügt. ${ }^{17}$

$14 \quad$ Neckel (1986), S. 595. Vgl auch Szasz (1986), S. 211.

15 Roland Roth: Eine korrupte Republik? Konturen politischer Korruption in der Bundesrepublik, in: Rolf Ebbinghausen / Sighard Neckel (Hg.): Anatomie des politischen Skandals, Frankfurt a.M. 1989, S. 201-233, S. 203.

16 Zum Unterschied zwischen journalistischer und publizistischer Vermittlungsstrategie vgl. ausführlich Kap. III.3., S. 154ff.

17

Vgl. Hitzler (1987), S. 24. Molotch/Lester, die den Skandal als eine Form eines öffentlichen Ereignisses betrachten, sehen für alle öffentlichen Ereignisse drei Rollen vor: news promoters (Primärquellen), news assemblers (Medien) und news consumers (Rezipienten). Da ihre Absicht darin liegt, eine Typologie von Ereignissen vorzulegen, schenken sie den Rollen der am 
Der 'publizistische' Umgang mit Skandalen ist geprägt von Parteilichkeit. Entsprechend schlägt sich der Publizist entweder auf die Seite des Skandalierers oder er ergreift Partei für den Skandalierten. In beiden Fällen bedient er sich der gleichen Mechanismen: Einseitige oder partielle Vermittlung von Gesprächsrepräsentanten, unterschiedliche Vorgehensweisen bei der Selektion und Transformation der genehmen und nicht genehmen Aussagen sowie Beigabe eigener Wertungen. Im ersteren Fall - Identifikation mit der Position des Skandalierers - wird er dabei bemüht sein, alles zu tun, um das Skandalöse der (vermeintlichen oder tatsächlichen) Normverletzung zu betonen und hervorzuheben und die Empörung zu schüren, sei es durch eigene Stellungnahmen oder durch die Vermittlung passender Stimmen. Im letzteren Fall Unterstützung des Skandalierten - wird er Maßnahmen ergreifen, die geeignet sind, Skandalrufe zu unterdrücken, oder (wenn dies nicht möglich ist) sie mindestens abzuschwächen und zu relativieren.

Daß der Publizist allein die Rolle des Skandalierers einnimmt, wäre ein Sonderfall der Parteinahme: in diesem Fall würde er seine eigenen Interessen vertreten. Diese können etwa darin bestehen, eine Auflagensteigerung zu erreichen oder Zuwachs an "Ruhm", der mit der "Aufdeckung" verknüpft ist, zu erwerben. ${ }^{18}$ In diesem Fall gilt:

Skandal beteiligten Personen keine weitere Aufmerksamkeit. (Vgl. Harvey Molotch / Marilyn Lester: News as Purposive Behavior: On the Strategic Use of Routine Events, Accidents, and Scandals, in: American Sociological Review 39/1974, S. 101-112, S. 104ff.) Auch Moodie weist dem Skandalierten keine eigene Rolle zu und benennt als erforderliche Positionen ebenfalls Informanten, Vermittlungskanäle und Publikum. (Vgl. Graeme C. Moodie: On Political Scandal and Corruption, in: Government and Opposition 15/1980, S. 208-222, S. 216.)

18 Eine "künstliche" Skandalisierung bestimmter Ereignisse zählt nach Auffassung von Fabris insbesondere bei Boulevardzeitungen "zu den zentralen Elementen einer auf die Erringung beherrschender Marktstellung ausgerichteten Marketingstrategie." (Hans Heinz Fabris: Massenmedien - Instrumente der "Skandalisierung" oder "Vierte Gewalt"? Zum Kontrollpotential der Medien, in: Christian Brünner (Hrsg.): Korruption und Kontrolle, Wien 1981, S. 239-264, S. 249. Siehe dazu auch Herbert Riehl-Heyse: "Die segensreiche Wirkung auf die Steigerung der Auflage entfaltet ein Skandal auch dann, wenn die eigentlichen Ärgernisse gar nicht entdeckt werden und statt dessen ein hausgemachtes Affärchen künstlich am Leben erhalten wird." (Das nützliche Ärgernis. Warum wir Skandale brauchen, in: Süddeutsche Zeitung vom 10./11. Juli 1982.) 
"Die Medien 'vermitteln' dabei nicht nur den skandalträchtigen Zusammenhang an die Öffentlichkeit; vielmehr gestalten sie ihn auch nicht selten gemäß ihren eigenen Funktionsgesetzen um. Auch kommt es nicht selten vor, daß sich bestimmte Medien auf die Geburtshilfe von Skandalen spezialisieren..., dabei aber keineswegs immer einen Treffer landen." 19

Allerdings ist davon auszugehen, daß Skandale selten erfolgreich sind, wenn ausschließlich die Vermittlungsinstanzen als Skandalierer agieren, denn

"kollektive Empörung und große Skandale entstehen im Widerstreit von Wertgemeinschaften genauer: dann, wenn eine enthüllte Normverletzung wie durch ein Brennglas einen schon lange schwelenden Konflikt zwischen zwei grundlegenden Werten aufflammen läßt. (...) Im Widerstreit liegende Werte scharen Wertgemeinschaften um sich, die auf den nächsten Skandal warten, um der Gegenseite eine Empörungsniederlage zufügen zu können." ${ }^{20}$

Die Verbindung zu Konflikten knüpfen auch Kepplinger/Hartung. Sie verstehen Skandale als Sonderfälle von publizistischen Konflikten und öffentlichen Krisen. Publizistische Konflikte sind definiert als "Auseinandersetzungen zwischen mindestens zwei Kontrahenten, die mit Hilfe der Massenmedien vor Publikum ausgetragen werden"; öffentliche Krisen sind "Auseinandersetzungen anläßlich schwerer Bedrohungen und Schäden". ${ }^{21}$ Während bei publizistischen

19 Wilfried von Bredow: Legitimation durch Empörung. Vorüberlegung zu einer politischen Theorie des Skandals, in: Schoeps (1992), S. 190-208, S. 198.

20 Karl Otto Hondrich: Skandalmärkte und Skandalkultur, in: Max Haller / Hans-Joachim Hoffmann-Nowotny / Wolfgang Zapf (Hg.): Kultur und Gesellschaft. Verhandlungen des 24. Deutschen Soziologentags, des 11. Österreichischen Soziologentags und des 8. Kongresses der Schweizerischen Gesellschaft in Zürich 1988, Frankfurt a.M./New York 1989, S. 575-586, S. 579 .

21 Kepplinger / Hartung (1993), S. 5f. Der Begriff 'publizistisch' wird hier im umgangssprachlichen Sinn verwendet. Siehe auch Hans Mathias Kepplinger: Publizistische Konflikte. Begriffe, Ansätze, Ergebnisse, in: Friedhelm Neidhardt (Hrsg.): Öffentlichkeit, Öffentliche Meinung, soziale Bewegungen, Opladen 1994 (=Sonderheft 34 der Kölner Zeitschrift für Soziologie und Sozialpsychologie), S. 214-233. 
Konflikten gegensätzliche Positionen von zwei Lagern mit ähnlichem Gewicht vertreten werden, gibt es bei öffentlichen Krisen nur eine legitime Position. Strittig ist hier (im Gegensatz zum Konflikt) nicht die Sache selbst, sondern die Konsequenzen, die daraus abgeleitet werden. Bei Skandalen gibt es ebenfalls nur eine legitime Position; gleichzeitig besteht Einigkeit hinsichtlich der Konsequenzen: es kommt nur die Verurteilung der Schuldigen in Frage. ${ }^{22}$ Bei Skandalen stehen sich also (im Unterschied zu publizistischen Konflikten) keine gleichwertigen Gegner gegenüber; vielmehr sind "die Rollen der Guten und der Bösen ... von Beginn an vergeben."23

Gemeinsamkeiten der drei Formen der Auseinandersetzung bestehen laut Kepplinger/Hartung hinsichtlich des zeitlichen Ablaufs, der Themenstruktur, des betroffenen Personenkreises und der Behandlung durch die Medien. Ihrer Auffassung nach entwickeln Vermittler in jedem Fall eigene Sichtweisen und Vorstellungen. Die Berichterstattung ist in der Folge geprägt von einer 'Instrumentellen Aktualisierung' der Informationen, das heißt: Informationen, die eigene Sichtweisen stützen, werden hochgespielt, und Informationen, die der eigenen Sichtweise zuwiderlaufen, werden in den Hintergrund gedrängt. Das führt dazu, daß Akteure, die eine andere Sicht der Dinge vertreten, nur geringe Chancen haben, ihre Position unverfälscht zu präsentieren. Statt dessen wird über sie nur bruchstückhaft und wertend berichtet. ${ }^{24}$

Kepplinger/Hartung sehen also als gegeben an, daß Vermittler sowohl im

$22 \quad$ Vgl. Kepplinger / Hartung (1993), S. 6.

23 Vgl. Kepplinger / Hartung (1993), S. 9. Zur Illustrierung des Unterschieds zwischen publizistischem Konflikt und Skandal siehe den Vergleich der Berichterstattung über die 'Fälle' Späth und Stolpe. (Hans Mathias Kepplinger et al.: Am Pranger: Der Fall Späth und der Fall Stolpe, in: Wolfgang Donsbach et al.: Beziehungsspiele - Medien und Politik in der öffentlichen Diskussion. Fallstudien und Analysen, Gütersloh 1993, S. 159-220.)

Vgl. Kepplinger / Hartung (1993), S. 7f. Siehe ferner Hans Mathias Kepplinger et al.: Instrumentelle Aktualisierung. Grundlagen einer Theorie kognitiv-affektiver Medienwirkungen, in: Winfried Schulz (Hrsg.): Medienwirkungen. Einflüsse von Presse, Radio und Fernsehen auf Individuum und Gesellschaft. Untersuchungen im Schwerpunktprogramm "Publizistische Medienwirkungen", Weinheim 1992, S. 161-189. 
Fall von publizistischen Konflikten als auch bei Skandalen ausschließlich publizistisch agieren. Diese einseitige Rollenzuweisung steht im Widerspruch zur zeitungswissenschaftlichen Auffassung, wonach Vermittler sich vom Prinzip der Unparteilichkeit oder der Parteilichkeit leiten lassen können. Welche Vermittlungsstrategie sie einschlagen, ist von Fall zu Fall und von Medium zu Medium zu prüfen und kann nicht von vorneherein unterstellt werden.

\subsection{Skandale und die Glaubwürdigkeit der Beteiligten}

Die Rolle der Glaubwürdigkeit wird im Zusammenhang mit Skandalen kaum thematisiert, sieht man von pauschalen Hinweisen, daß Skandale die Glaubwürdigkeit von Politikern mindern, ab. ${ }^{25}$ Faßt man Skandale als eine Form öffentlicher Konflikte auf, ist aber davon auszugehen, daß die Glaubwürdigkeit der beteiligten Konfliktparteien eine zentrale Rolle spielt.

Laut Kepplinger haben Akteure im Rahmen von Konflikten zwei Strategien zur Verfügung:

"Bei defensivem Vorgehen versucht der Kontrahent, sein eigenes Erscheinungsbild zu verbessern, indem er zum Beispiel seine Leistungen herausstellt, negativen Urteilen widerspricht und falsche Behauptungen richtigstellt. Bei offensivem Vorgehen versucht der Kontrahent, das Erscheinungsbild seines Widersachers zu verschlechtern, indem er zum Beispiel seine Kompetenz in Frage stellt oder seine Glaubwürdigkeit erschüttert." 26

Bei Konflikten geht es also nicht ausschließlich um Sachargumente, sondern auch darum, in welchem Licht die Akteure erscheinen. Jeder wird versuchen, von der eigenen Seite ein möglichst positives Bild zu prägen und gegebenenfalls dem Gegner negative Eigenschaften zuzuschreiben. Luhmann verweist

25 Vgl. etwa Käsler et al. (1991), S. 58f.

26 Kepplinger (1994), S. 221. [Hervorhebung im Original.] 
darauf, daß "alles sozial einsehbare Handeln ... neben seinem unmittelbarem Sinnbezug auf Situation und Zweck zugleich Selbstdarstellung des Handelnden unter dem Gesichtspunkt seiner Vertrauenswürdigkeit" ist. ${ }^{27}$ Laut Schütz sind "politische Skandale und Affären quasi der Extremfall für die Selbstdarstellung von Politikern". ${ }^{28}$ Sie benennt drei Formen der Selbstdarstellung: Zur offensiven Selbstdarstellung gehört - wie bei Kepplinger - die direkte oder indirekte Abwertung des politischen Gegners; dazu zählen aber auch alle Versuche, das Gespräch zu dominieren. Dies geschieht durch Kritik am Fragesteller, durch direkte oder indirekte Antwortverweigerung, durch Festlegung des Gesprächsthemas oder dadurch, daß man die eigene Meinung ohne nähere Begründung behauptet und bekräftigt. ${ }^{29}$ Der defensiven Selbstdarstellung werden alle Reaktionen auf Angriffe zugeordnet. Die stufenweise Steigerung von Formen defensiver Selbstdarstellung läßt sich etwa dann beobachten, "wenn im Verlauf politischer Skandale immer mehr belastende Informationen bekannt werden". ${ }^{30}$ Die Verteidigungshaltung drückt sich aus in Kommunikationsakten wie Leugnen, Umdeuten von Ereignissen oder Rechtfertigen. Zur defensiven Strategie gehört auch das Ablehnen der Verantwortung (etwa durch Benennung eines 'Sündenbocks') oder die Reduktion von Verantwortung (durch Anführen 'mildernder Umstände'). Ferner können Angriffe dadurch abgewehrt werden, daß die eigenen Handlungen als "normales Verhalten", das bei anderen in ähnlicher Weise zu beobachten ist, gedeutet werden (horizontaler Vergleich) oder dadurch, daß das eigene Verhalten im

27 Niklas Luhmann: Vertrauen. Ein Mechanismus der Reduktion sozialer Komplexität, Stuttgart ${ }^{3} 1989$, S. 41 .

28 Astrid Schütz: Politik oder Selbstdarstellung? Beispiele von Politikerauftritten, in: Michael Jäckel / Peter Winterhoff-Spurk (Hg.): Politik und Medien. Analysen zur Entwicklung der politischen Kommunikation, Berlin 1994, S. 193-209, S. 194. Siehe dazu auch ausführlich Astrid Schütz: Selbstdarstellung von Politikern. Analyse von Wahlkampfauftritten, Weinheim 1992.

29 Vgl. Schütz (1994), S. 196ff.

$30 \quad$ Schütz (1994), S. 199. 
Vergleich zu anderen als relativ positiv bezeichnet wird (vertikaler Vergleich). ${ }^{31}$ Gerade, wenn es um Skandalisierungen geht, führt eine rein defensive Haltung zu einer Minderung der Glaubwürdigkeit. ${ }^{32}$

Die Kategorie 'defensive Selbstdarstellung' umfaßt damit nur einen Teilbereich dessen, was Kepplinger als 'defensives Vorgehen' bezeichnet; dieser schließt auch die vorteilhafte Präsentation des Selbstbildes mit ein. Alle Aspekte der positiven Darstellung der eigenen Person, in denen keine aggressiven oder verteidigenden Elemente enthalten sind, bezeichnet Schütz dagegen als assertive Selbstdarstellung. Diese Form der Selbstdarstellung soll hier etwas ausführlicher betrachtet werden, da sie in den meisten Bereichen eng mit verschiedenen Dimensionen der Glaubwürdigkeit verknüpft ist.

Der Eindruck von Kompetenz wird vermittelt, indem man Fakten oder detaillierte Erklärungen präsentiert, aber auch dadurch, daß Belege aller Art geliefert werden, sei es, daß man sich auf berühmte Dritte oder Experten beruft oder daß man den eigenen Status oder erreichte Erfolge als Beleg anführt. ${ }^{33}$

Der Schaffung von Vertrauenswürdigkeit dient die Strategie der Selbstöffnung: "Nach der Norm der Reziprozität erlangt Vertrauen, wer etwas von sich selbst preisgibt und sich dem Gegenüber öffnet." ${ }^{34}$ Die Selbstöffnung kann darin bestehen, Persönliches oder Privates zu erzählen oder emotionale Beteiligung erkennen zu lassen oder darin, Fehler einzugestehen und Selbstkritik zu üben. ${ }^{35}$ Vertrauenswürdigkeit kann ferner angestrebt werden, indem

31 Vgl. Schütz (1994), S. 199ff. Siehe auch Astrid Schütz: Mehr oder weniger zugeben: Stufen defensiver Selbstdarstellung, in: Heinz-Ulrich Kohr / Massimo Martini / Angelika Kohr (Hg.): Macht und Bewußtsein. Europäische Beiträge zur Politischen Psychologie, Weinheim 1990, S. 131-141.

32 Vgl. Szasz (1986), S. 212.

33 Vgl. Schütz (1994), S. 204ff. Siehe dazu auch Abschnitt II.C.6d) dieser Arbeit (S. 82ff.).

34 Schütz (1994), S. 203.

35 Vgl. Schütz (1994), S. 203f. Zur Selbstöffnung siehe auch: Franz Petermann: Psychologie des Vertrauens, München ${ }^{2} 1992$, S. 68f. 
ein Sprecher betont, "daß sein Handeln von ethischen Werthaltungen und moralischen Standards geleitet wird". ${ }^{36}$ Übertrieben positive Selbstdarstellung wird allerdings als unehrlich betrachtet, eine gewisse Zurückhaltung scheint empfehlenswert. ${ }^{37}$

Eine weitere Form assertiver Selbstdarstellung ist die Akzentuierung der Rolle als Bürgervertreter. Dadurch, daß die Wahrung der Bürgerinteressen hervorgehoben wird oder dadurch, daß Politiker sich als "Menschen wie Du und ich" präsentieren, wird die Ähnlichkeit mit der Wählerschaft betont. ${ }^{38}$ Auch die Kategorie 'Beziehungspflege' dient der Betonung von Ähnlichkeit, allerdings ist damit ausschließlich der Versuch gemeint, Gemeinsamkeiten mit einem interviewenden Journalisten herzustellen. Gegenüber dem Publikum wird damit gleichzeitig der Eindruck vermittelt, freundlich und gelassen zu sein. ${ }^{39}$ Daran wird deutlich, daß die Strategien, die darauf abzielen, Ähnlichkeiten zu suggerieren oder Vertrauenswürdigkeit herzustellen, gleichzeitig geeignet sind, Sympathie hervorzurufen.

36 Schütz (1994), S. 202. Als "Extrembeispiel" dieser Selbstdarstellungsform wird hier das Ehrenwort von Uwe Barschel genannt.

37

Vgl. Michael D. Robinson / Joel T. Johnson / Stephanie A. Shields: On the Advantages of Modesty: The Benefits of a Balanced Self-Presentation, in: Communication Research 22/1995, S. 575-591, S. 586

38

Vgl. Schütz (1994), S. 202f. Schütz siedelt unter dieser Kategorie zusätzlich die Strategien "Versprechungen" und "Optimismus vermitteln" an, die mit Glaubwürdigkeit nicht in unmittelbarem Zusammenhang stehen. Als Beispiel für die Präsentation als "ganz normaler Mitbürger" nennt sie Filmszenen, die Uwe Barschel beim Familienfrühstück und beim Fußballspielen mit seinem Sohn zeigen. (Vgl. ebd. S. 203.) Perloff berichtet Vergleichbares vom Wahlkampf in den USA: "During the summer of 1992, Bill Clinton and Al Gore launched their presidential campaign by embarking a bus trip across Middle America. Clinton and Gore wore short sleeve shirts and Levis. They threw the football around an open field. They talked about their small town backgrounds." (Richard M. Perloff: The Dynamics of Persuasion, Hilsdale N.J. 1993, S. 145f.) 


\subsection{Zwischenbilanz}

Ein Skandal entsteht, wenn eine Normverletzung öffentliche Empörung hervorruft. Als 'politisch' ist er einzustufen, wenn die beteiligten Akteure (Skandalierer oder - in der Regel - Skandalierte) politische Mandatsträger oder Angehörige von Administrationen oder Parteien sind. Zur Skandal-Triade gehört neben den "Hauptrollen", die von Skandalierer und Skandaliertem eingenommen werden, ein Publikum, das eine Reaktion auf eine Normverletzung zeigt.

Um einen politischen Skandal öffentlich zu machen, sind in modernen Gesellschaften Massenmedien erforderlich. Je nachdem, welche Vermittlungsstrategie diese wählen, können sie sich als neutrale Vermittler betätigen, die alle Beteiligten angemessen zu Wort kommen lassen, oder sie können für eine der beiden Seiten - Skandalierer oder Skandalierte - Partei ergreifen und diese bevorzugt behandeln.

Ein Skandal kann als Sonderfall eines öffentlichen Konflikts aufgefaßt werden. Bei öffentlichen Konflikten kommt es nicht zuletzt darauf an, welche Gelegenheit den Akteuren zur Selbstdarstellung gegeben wird. Neben offensiven und defensiven Selbstdarstellungsformen steht insbesondere die positive, assertive Darstellung der eigenen Person in engem Zusammenhang mit den Glaubwürdigkeitsdimensionen 'Kompetenz', 'Vertrauenswürdigkeit', 'Ähnlichkeit' und 'Sympathie'. Eine Möglichkeit publizistischer Einflußnahme besteht mithin darin, bei unliebsamen Akteuren die Chancen zur Selbstdarstellung zu beschneiden bzw. Vertretern einer genehmen Position verstärkt Gelegenheit dazu einzuräumen. Zusätzlich kann das vermittelte Selbstbild durch Stimmen anderer oder durch eigene Wertungen kontrastiert oder bestätigt werden. Bei einer Analyse der Berichterstattung ist also darauf zu achten, wie die beteiligten Akteure insgesamt dargestellt werden und wer für welche Darstellungsaspekte verantwortlich ist. 


\section{Zusammenfassung und Ausblick}

Worauf stützen sich Urteile über die Glaubwürdigkeit von Personen? Auf welche Weise manifestieren sich Glaubwürdigkeitskriterien in den Massenmedien? Und schließlich: Welche Rolle spielt die Glaubwürdigkeit der beteiligten Akteure bei der Berichterstattung über politische Skandale? Welchen Einfluß nehmen die Vermittler bei der Zuschreibung von Glaubwürdigkeit? Diese Fragen sollen im folgenden komprimierten Überblick beantwortet werden.

Als illustrierende Fallbeispiele dienen drei politische Skandale aus der jüngeren Vergangenheit: Die 'Briefbogen-Affäre', die Anfang 1993 zum Rücktritt des Bundeswirtschaftsministers Jürgen Möllemann (F.D.P.) führte, die 'Putzfrauen-Affäre', die in der Verknüpfung mit weiteren Vorwürfen 1993 den Rücktritt von Bundesverkehrsminister Günther Krause (CDU) verursachte und die 'Kieler Affäre', bei der es um Machenschaften im Verlauf des Wahlkampfes zur Landtagswahl in Schleswig-Holstein ging, und die mit dem Tod des Hauptskandalierten, Ministerpräsident Uwe Barschel (CDU), im Oktober 1987 nur ein vorläufiges Ende fand.

Untersuchungsobjekte waren in allen Fällen die überregionalen Abonnementzeitungen 'Die Welt' (WELT), 'Frankfurter Allgemeine Zeitung' (FAZ), 'Süddeutsche Zeitung' (SZ) und 'Frankfurter Rundschau' (FR), die laut Kepplinger ein publizistisches Spektrum darstellen, in dem sich das Spektrum der Parteien und Interessengruppen spiegelt. ${ }^{1}$ Um zu prüfen, ob in 
verschiedenen Medientypen unterschiedliche Strategien hinsichtlich der Beschäftigung mit Skandalen zu beobachten sind, wurden ferner die überregionale Boulevardzeitung 'Bild' (BILD) und das Nachrichtenmagazin 'Der Spiegel' (SPIEGEL) in die Betrachtung miteinbezogen. bei der Vercodung berücksichtigt wurden alle Textbeiträge, die sich im jeweiligen Untersuchungszeitraum mit den Hauptskandalierten und/oder den von ihnen begangenen Normverstößen beschäftigten. ${ }^{2}$

\subsection{Befunde der Glaubwürdigkeitsforschung}

Der Frage nach der Glaubwürdigkeit von Personen haben sich drei unterschiedliche Forschungsperspektiven angenommen. Die verhaltensorientierte Glaubwürdigkeitsforschung sucht nach Indizien, die es ermöglichen, aufgrund des nonverbalen und extralinguistischen Verhaltens eines Sprechers, wahre von falschen Aussagen zu unterscheiden. Diese Diagnose wird dadurch erschwert, daß Abweichungen im Verhalten (erhöhte Lidschlagfrequenz, vermehrte Körperbewegungen, Reaktionslatenzen, Verzögerungen im Sprechverlauf, Sprechstörungen oder Vermeiden des Blickkontaktes) nicht schon eindeutige Indikatoren für lügenhaftes Verhalten sind. Vielmehr verweisen sie auf einen allgemeinen Erregungszustand und können ebenso auftreten, wenn der Sprecher ärgerlich, ängstlich oder nervös ist. In die Deutung miteinbezogen werden müssen zudem die individuellen Eigenheiten im Auftreten einer Person sowie die Kommunikationssituation.

Inhaltsanalyse der Abendnachrichten und politischen Magazine, Freiburg/München 1985, S. 2428. Die Redaktionelle Linie der WELT wird dabei als "ausgeprägt rechts", die der FAZ als "gemäßigt rechts", die der SZ als "gemäßigt links" und die der FR als "ausgeprägt links" eingestuft.

2

Im Fall Barschel, der bis heute nicht als abgeschlossen bezeichnet werden kann, wurde lediglich die erste Phase (bis zwei Wochen nach Barschels Tod) berücksichtigt; Untersuchungszeitraum war also der 7. September bis 26. Oktober 1987. Die beiden anderen Affären wurden jeweils vollständig bis zum Rücktritt des Hauptskandalierten erfaßt; der Untersuchungszeitraum reichte im Fall Möllemann vom 18. Dezember 1992 bis zum 6. Januar 1993, im Fall Krause vom 22. März bis 10. Mai 1993. 
Aufgrund dieser Unsicherheiten ist es nicht verwunderlich, daß in den meisten durchgeführten Experimenten die Anzahl der richtig erkannten Täuschungen nur wenig über dem Zufallsbereich liegt, sich also andere Personen - insbesondere, wenn sie den Sprecher nicht kennen - als wenig brauchbare "Lügendetektoren" erweisen. Dennoch ergaben Befragungen, daß im Bewußtsein der Menschen ein festgefügtes Stereotyp eines Lügners existiert, welches sich auf die nämlichen nonverbalen und extralinguistischen Verhaltensweisen stützt und von Rezipienten zur Einschätzung des Wahrheitsgehaltes einer Aussage herangezogen wird. Aus diesem Grund sollten die Indikatoren, die die verhaltensorientierte Forschungsrichtung bereitstellt, in eine Inhaltsanalyse integriert werden.

Die inhaltsorientierte Glaubwürdigkeitsbeurteilung will dazu beitragen, wahre von falschen Zeugenaussagen zu unterscheiden. Das Augenmerk gilt dabei weniger dem Sprecher selbst, sondern vorwiegend seiner Aussage. Geprüft werden Detailreichtum, Schlüssigkeit und Widerspruchsfreiheit mit früheren eigenen Aussagen oder den Aussagen anderer. In der Inhaltsanalyse muß sich diese Prüfung auf die "Aussagen über die Aussagen" verlagern, also darauf, wie die Aussage eines Sprechers von seiten Dritter beurteilt wird. Es geht also nicht darum, den tatsächlichen Wahrheitsgehalt zu ermitteln, sondern darum zu ergründen, ob der Wahrheitsgehalt angezweifelt oder untermauert wird.

Im Zusammenhang mit der Erforschung von Einstellungen und Einstellungsänderungen gerät die quellen- und kontextorientierte Glaubwürdigkeitsbeurteilung in den Blickpunkt. Dabei wird hinterfragt, welche verhaltensunabhängigen Merkmale einer Quelle dazu führen, daß ihr Glaubwürdigkeit zugeschrieben wird und welchen Einfluß die Kommunikationssituation auf eine solche Zuschreibung hat.

Zahllose Laborexperimente erbrachten als zentrale Dimensionen Kompetenz und Vertrauenswürdigkeit. Eine Quelle wird als kompetent erachtet, wenn sie über Wissen um einen Gegenstand verfügt, Qualifikationen oder Erfahrung aufweist, als intelligent gilt oder eine Führungsposition innehat. 
'Vertrauenswürdigkeit' mißt sich einerseits an der Ehrlichkeit oder Aufrichtigkeit einer Quelle und andererseits an ihrer Unparteilichkeit, Unabhängigkeit und Selbstlosigkeit. Auch der Kommunikationskontext wirkt sich auf die 'Vertrauenswürdigkeit' aus: Ein ausdrücklicher Hinweis auf die Beeinflussungsabsicht der Quelle (Forewarning) mindert das Vertrauen; das Belauschen einer Quelle, die scheinbar nichts von ihrer Zuhörerschaft ahnt (Overhearing) erhöht das Vertrauen, da in diesem Fall eine Beeinflussungsabsicht ausgeschlossen wird.

Einstellungsänderungen führt ein Sprecher auch herbei, wenn er auf andere Weise seine Glaubwürdigkeit erhöht oder stabilisiert: indem er auf Ähnlichkeiten mit seinen Zuhörern oder Rezipienten verweist, die sich auf die Gruppenzugehörigkeit oder auf Einstellungen und Werthaltungen beziehen können. Vor allem, wenn solche konkreten Kriterien fehlen, können Sympathie oder physische Attraktivität ausschlaggebend für die Zuschreibung von Glaubwürdigkeit sein. Bedeutsam ist schließlich auch die Dynamik, die ein Sprecher vermittelt, sei es durch den Eindruck von Aktivität und Stärke, den er hervorruft, oder durch sein Sprechverhalten.

\subsection{Die Glaubwürdigkeit von Akteuren: Operationalisierung und Ergebnisse}

Wie anhand des Forschungsüberblicks deutlich wurde, ist die Glaubwürdigkeit von Quellen bisher ausschließlich mittels experimenteller Versuchsanordnungen oder Befragungen ermittelt worden. Obgleich vereinzelt Zeitungsartikel als Stimulusmaterial Verwendung fanden, mangelt es an einer kontinuierlichen Betrachtung der Glaubwürdigkeit von Quellen, die indirekt, via Massenmedien, vermittelt werden. Eine Adaption der Erkenntnisse auf inhaltsanalytische Verfahren fand bisher nicht statt. Bei einer konsequenten Trennung von Vermittlungs- und Mitteilungsprozeß läßt sich jedoch durchaus mittels einer Inhaltsanalyse die Frage beantworten, ob und auf welche Weise den 
Kommunikationspartnern im Rahmen der Massenkommunikation Glaubwürdigkeit zu- oder abgesprochen wird.

Wie eingangs erläutert, kann dabei ausschließlich betrachtet werden, welche für die Glaubwürdigkeit relevanten Zuschreibungen beobachtet werden können. Rückschlüsse, ob diese Zuschreibungen bei den Rezipienten tatsächlich den Eindruck von Glaubwürdigkeit oder Unglaubwürdigkeit erzeugen, entziehen sich der inhaltsanalytischen Beobachtung und sind daher nur bedingt möglich.

Die übergeordnete Frage, die mittels Inhaltsanalyse geklärt werden kann, lautet also: Welche Glaubwürdigkeitsmerkmale werden den beteiligten Kommunikationspartnern zugewiesen und von wem stammen die Kennzeichnungen? In diesem Zusammenhang werden die folgenden Teilfragen relevant:

* $\quad$ Sind die Grundbedingungen für die Orientierung der Rezipienten erfüllt, das heißt, werden die beteiligten Partner ausreichend vorgestellt und hinsichtlich ihrer sachlichen Herkunft sowie ihrer etwaigen Parteizugehörigkeit verortet?

* Gibt es darüberhinaus im Rahmen der Partnervorstellung zusätzliche 'Vertrauensinformationen', die Aufschluß geben über Kompetenz und Vertrauenswürdigkeit der Akteure sowie das Ausmaß der Sozialen Billigung ihrer Positionen?

* $\quad$ Gibt es schließlich weitergehende Hinweise auf die Art der persönlichen Präsentation (Dynamik) und Sympathie oder Attraktivität der Akteure?

Einer der wesentlichen Orientierungspunkte für den Leser ist etwa das Wissen darum, wer etwas sagt. Entsprechend enthält jede zeitungswissenschaftliche Inhaltsanalyse eine Reihe von Variablen, mit deren Hilfe die Identität von Ausgangspartnern bestimmt wird; einige dieser Variablen lassen gleichzeitig Rückschlüsse auf die zugeschriebene Glaubwürdigkeit zu. Die Variable Transparenz beantwortet dabei die Frage, ob die vermittelten Personen oder 
Institutionen namentlich 'genannt' und damit eindeutig identifizierbar, 'anonym' oder 'ungenannt' sind. Die Ausprägung 'ungenannt' wird vergeben, wenn keinerlei Hinweis auf einen Ausgangspartner vorliegt, die vermittelten Informationen jedoch Sachverhalte enthalten, die dem Vermittler nicht ohne weiteres zugänglich sein können. 'Anonym' schließlich bedeutet hier vorerst nur, daß keine Namensnennung vorliegt. Das Ausmaß der Anonymität läßt sich in Kombination mit weiteren Variablen konkretisieren. Dazu gehört die geographische Herkunft ebenso wie insbesondere die sachliche Herkunft, die darüber Auskunft gibt, aus welchen gesellschaftlichen Bereichen ein Ausgangspartner kommt, sowie die Variable Parteizugehörigkeit. ${ }^{3}$

Die Dimension 'Ähnlichkeit', die in den Quellenexperimenten gewisse Bedeutung hat, ließ sich für die Inhaltsanalyse nicht operationalisieren, da mit dieser Dimension eine Beziehung zwischen Rezipient und Quelle hergestellt wird. Die Wahrnehmung von 'Ähnlichkeit' ist abhängig von der individuellen Einschätzung eines Rezipienten und seinen Einstellungen oder Gruppenzugehörigkeiten. Daher gibt es keine feststehenden Merkmale, aufgrund derer einer Quelle 'Ähnlichkeit' zugeschrieben wird. Geprüft werden kann lediglich, ob den Rezipienten Hinweise geliefert werden, denen sie Rückschlüsse auf Ähnlichkeiten entnehmen können. Dies ist etwa der Fall, wenn eine Parteizugehörigkeit mitgeteilt wird - auch wenn im Hinblick auf die Glaubwürdigkeit jeder Rezipient aus dieser Information unterschiedliche Schlüsse zieht. Bei Skandalierern und Skandalierten wurde diese Orientierungshilfe in der Regel geliefert; Aussageträger ohne fixierbare Skandal-Rolle, die großen Anteil am vermittelten Gespräch haben, sind dagegen hinsichtlich ihrer Parteizugehörigkeit oft nicht zu verorten, wobei offen bleiben muß, ob sie tatsächlich keiner Partei angehören oder ob die Zugehörigkeit nicht mitgeteilt wird.

Die Variablen Transparenz, geographische Herkunft, sachliche Herkunft und Parteizugehörigkeit liefern Basisinformationen, die in zeitungswissenschaftlichen Inhaltsanalysen zum Erhebungsstandard gehören. Sie tragen nur mittelbar zur Glaubwürdigkeitszuschreibung ein, indem sie die Aussageträger identifizieren. 
Es ist davon auszugehen, daß eine Reihe von Glaubwürdigkeitszuschreibungen mittels wertender Charakterisierungen erfolgt. In den Quellen-Experimenten der verhaltensorientierten Glaubwürdigkeitsbeurteilung bediente man sich für die Erhebung solcher Charakterisierungen Semantischer Differentiale oder Polaritätenprofile. Eine Reihe von Untersuchungen hat gezeigt, daß die Adaption dieses Instruments auch für die Inhaltsanalyse möglich ist. ${ }^{4}$ Hierbei wurde bisher kein standardisiertes Profil zugrundegelegt, "da davon auszugehen ist, daß für jeden Untersuchungsgegenstand zumindest partiell eigene Einschätzungen und Bewertungen vorliegen". ${ }^{5}$ Daher wurde bislang für jede Untersuchung ein individuelles Profil konstruiert. ${ }^{6} \mathrm{Zu}$ diesem Zweck wurde

4 Erstmals erprobt und beschrieben ist die Vorgehensweise bei Hans Wagner: Wirklichkeits-Präparate? Das Medikamenten-Bild in deutschen Printmedien, in: Hans Wagner / Heinz Starkulla (Hg.): Medizin \& Medien, München 1984, S. 10-104, S. 77ff.

Ursula E. Koch / Ute Nawratil / Detlef Schröter: Sommer 1789: Französische Revolution und preußische Zeitungsberichte, in: Holger Böning (Hrsg.): Französische Revolution und deutsche Öffentlichkeit, München u.a. 1992, S. 199-249, S. 221.

Das Instrument fand Verwendung in einer Reihe von Untersuchungen, insbesondere in Abschlußarbeiten. Vgl. z.B. Schröter (1995; Bewertungsobjekt: Unternehmen); Marie-Agnes Strack-Zimmermann: Bilder aus Amerika. Eine zeitungswissenschaftliche Studie über die USABerichterstattung im Zweiten Deutschen Fernsehen (ZDF), Frankfurt a.M. 1987 (Bewertungsobjekt: USA); Ute Nawratil: "Wenig Lärm um viel". Die Berichterstattung über Reproduktionsmedizin und Genforschung. Vergleichende Inhaltsanalyse, München 1987 (unveröffentlichte Magisterarbeit, Bewertungsobjekt: Gentechnologie); Christian Poitsch: Die Parteien vor der Wahl. Stereotypenuntersuchung zur Berichterstattung vor der Bundestagswahl 1987. Inhaltsanalyse, München 1988 (unveröffentlichte Magisterarbeit, Bewertungsobjekt: Parteien und Politiker); Norbert Haimerl: Schreiben sie wirklich alle dasselbe? Auf der Suche nach Konsonanz und Publizistischem Spektrum. Vergleichende Inhaltsanalyse der Berichterstattung von vier deutschen Tageszeitungen anläßlich des Reykjavik-Treffens von Ronald Reagan und Michail Gorbatschow im Oktober 1986, München 1988 (unveröffentlichte Magisterarbeit); Eckhard Mischke: "Flüsterjets und Donnervögel". Imageanalyse am Beispiel der Berichterstattung über den Flughafen München 2, München 1989 (unveröffentlichte Magisterarbeit, Bewertungsobjekt: Flughafen München II); Christiane Kröck: Das Deutschlandbild in der französischen Tagespresse des Elsaß am Beispiel der 'Dernières Nouvelles d'Alsace', München 1990 (unveröffentlichte Diplomarbeit, Bewertungsobjekt: Deutschland und Deutsche); Cathrin Hegner: 'Deutsche Ansichten'. Stereotype und die Darstellung von Ostdeutschen in 'Stern' und 'Bunte'. Eine vergleichende Inhaltsanalyse, München 1994 (unveröffentlichte Magisterarbeit, Bewertungsobjekt: Ostdeutsche). 
das zu untersuchende Textmaterial gesichtet, und es wurden alle Attribute notiert, die den Untersuchungsgegenstand kennzeichneten. "Berücksichtigt werden dabei sowohl rein sachliche Klassifikationen und Beschreibungen als auch beiläufige, zum Teil unbewußt verwendete Attributionen, subjektive Einschätzungen und Werturteile. ${ }^{7}$ Zur Strukturierung des Materials wurden sodann in einem weiteren Arbeitsschritt alle Synonyme oder sinnverwandten Attribute zu sogenannten 'Bedeutungshöfen' zusammengefaßt.

"Es ist dabei nicht zu vermeiden, daß solche 'Bedeutungshöfe' offene Ränder aufweisen, oder sich an ihren Rändern gelegentlich überlappen. Aber da jeder 'Bedeutungshof' nicht theoretischallgemein, sondern pragmatisch, d.h. ausschließlich durch alle ihm zugewiesenen Einzelattributionen definiert ist, ist das Verfahren objektivierbar und läßt später eindeutige Kodierungen zu." 8

Diese Bedeutungshöfe wurden dann mit einer 'Leitkennung' versehen, in der Regel ein Adjektiv, das alle im Hof enthaltenen Attribute möglichst prägnant zusammenfaßt. Durch die Gegenüberstellung antonymer Leitkennungen ergab sich schließlich das Polaritätenprofil. ${ }^{9}$ Bei der Untersuchung von 'Glaubwürdigkeit' kann auf diese umfangreichen Vorarbeiten verzichtet werden. Da sich das Konstrukt in verschiedene Dimensionen auflösen läßt und sich diese Dimensionen auch inhaltlich ableiten und bestimmen lassen, läßt sich stattdessen ein Universalprofil konstruieren, das den Dimensionen Rechnung trägt. Die Bedeutungshöfe, die im Hinblick auf Glaubwürdigkeit bedeutsam sind, können aus bestehenden Studien extrahiert und durch Gegensatzpaare, die im Rahmen der Persuasionsforschung in Semantischen Differentialen Verwendung

$7 \quad$ Koch / Nawratil / Schröter (1992), S. 222.

8 Hans Wagner: Der Name des Fortschritts heißt 'Erfolg', München 1986 (unveröffentlichtes Manuskript), S. 106.

Denkbar ist auch eine einfache Reihung, die dann einer Eigenschaftsliste entsprechen würde. Die Erfahrung zahlreicher Studien hat jedoch gezeigt, daß sich die Gegensätze sozusagen "von selbst" ergeben, also immer schon im Material zugrundeliegen. Lediglich in Ausnahmefällen ist es erforderlich, zu einzelnen Leitkennungen ein Antonym zu konstruieren. 
fanden, ergänzt werden. Grundlage für die Vercodung ist damit ein umfangreiches Wörterbuch, das alle sinn- und sachverwandten Zuschreibungen eines jeden Bedeutungshofes ausweist.

\subsubsection{Kompetenz}

In der Dimension 'Kompetenz' geht es darum, Hinweise zu finden, die Aufschluß darüber geben, ob die Person, die beurteilt wird, weiß, wovon sie spricht und ob sie über fachliche oder andere nötige Qualifikationen verfügt.

Solche Hinweise ergeben sich direkt aus der Vermittlung von Berufsbezeichnungen, Titeln oder ähnlichen Angaben, die in der Variable 'Herkunftskompetenz' erfaßt werden. Indirekte Rückschlüsse auf die Kompetenz ergeben sich zusätzlich aus der Vermittlung von Organisationszugehörigkeiten, speziell aus Informationen über das Innehaben von Führungspositionen oder über die Zugehörigkeit $\mathrm{zu}$ bestimmten Hierarchieebenen. ${ }^{10}$ Solche Informationen werden mit der Variable 'Organisationslegitimation' erfaßt. Zusätzlich ist zu prüfen, ob die Kompetenzmerkmale eines Aussageträgers in Verbindung stehen mit dem Thema, über das er spricht. Dies geschieht mit der Variable 'Kompetenzrelevanz'. ${ }^{11}$

Darüberhinaus sind weitere Zuschreibungen denkbar, durch die die Kompetenz des Bewertungsobjekts eine nähere Begründung erfährt. So ist festzuhalten, ob jemand explizit als 'Experte', 'Fachmann' oder 'Spezialist' bezeichnet wird beziehungsweise ob ihm solches Expertentum abgesprochen wird. ${ }^{12}$ Es kann erfaßt werden, ob dem Bewertungsobjekt 'Erfahrung' auf einem bestimmten Gebiet zu- oder abgesprochen wird und ob es über 'Insider-Wissen'

$10 \quad$ Vgl. Hovland / Janis / Kelley (1953), S. 21.

11 Die Variablen 'Herkunftskompetenz', 'Organisationslegitimation' und 'Kompetenzrelevanz' sind Bestandteile des Standardinstruments der zeitungswissenschaftlichen Inhaltsanalyse.

12 Zur Bedeutung von Experten für die Glaubwürdigkeit siehe Francis J. Di Vesta / Donald L. Meyer / Judson Mills: Confidence in an Expert as a Function of his Judgements, in: Human Relations 17/1964, S. 235-242. 
verfügt (etwa durch den Zugang zu Exklusiv-Informationen, durch Zeugenschaft oder intensive Bekanntschaft mit dem Bewertungsobjekt ${ }^{13}$ ). Ferner sind Hinweise auf 'Ehrungen' oder 'Verdienste' ebenso festzuhalten wie solche, die auf 'Anerkennung' der Fähigkeiten durch andere zielen. ${ }^{14}$

Schließlich sind für die Erfassung verschiedener Aspekte der Kompetenz im Polaritätenprofil acht Gegensatzpaare reserviert (kompetent-inkompetent, fähig-unfähig, richtig-verfehlt, realistisch-realitätsfern, geschickt-unbeholfen, klug-dumm, erfahren-unerfahren, planend-planlos).

Bei der Inhaltsanalyse erwies sich 'Kompetenz' nicht als zentrales Kriterium. Zwar werden förmliche Kompetenzmerkmale - in erster Linie für die Skandalierten - vermittelt, aber kaum durch weitergehende Hinweise verstärkt. Für Aussageträger ohne Skandal-Rolle werden häufiger keine Kompetenzmerkmale mitgeteilt. Anhaltspunkte, die den Expertenstatus untermau-

Die Bedeutsamkeit von Länge und Intensität einer persönlichen Bekanntschaft wird hervorgehoben bei: Michael H. Birnbaum / Rebecca Wong / Leighton K. Wong: Combining Information from Sources that Vary in Credibility, in: Memory \& Cognition 4/1976, S. 330336. Siehe dazu ergänzend auch: Michael H. Birnbaum / Steven E. Stegner: Source Credibility in Social Judgment: Bias, Expertise, and the Judge's Point of View, in: Journal of Personality and Social Psychology 37/1979, S. 48-74.

14 Den letztgenannten Kriterien mißt etwa Wild besondere Bedeutung zu, wenn er in einem Ratschlag an Laien formuliert: "'Erkunde die wissenschaftliche Reputation der Experten und räume einem international angesehenen Wissenschaftler einen Vertrauensvorsprung ein.' (...) Ein Professorentitel ist heute sicherlich kein ausreichendes Kriterium mehr. Brauchbarer ist die Mitgliedschaft in wissenschaftlichen Akademien, denn diese Gremien haben ihre Mitgliedszahlen kaum erweitert. Eine akademische Karriere, die sich ausschließlich innerhalb einer einzigen Einrichtung vollzogen hat, spricht gegen, eine Vielzahl von Rufen an verschiedene Hochschulen oder vergleichbare wissenschaftliche Einrichtungen für das Ansehen eines Wissenschaftlers. Das in diesem Zusammenhang beste Kriterium ist der internationale Bekanntheitsgrad, der sich etwa in der Wahl zum wirklichen oder korrespondierenden Mitglied ausländischer Akademien oder in der Zuerkennung internationaler Preise niederschlägt." (Wolfgang Wild: Kriterien der Glaubwürdigkeit von Expertenaussagen. Vortrag bei der öffentlichen Jahrestagung des Vereins der Freunde der Universität Regensburg e.V. am 29. Juli 1987 in Regensburg, o.O., o.J., S. 7.) Ähnliche Indikatoren für wissenschaftliche Reputation siehe auch bei Hans Peter Peters: Wissenschaftliche Experten in der öffentlichen Kommunikation über Technik, Umwelt und Risiken, in: Friedhelm Neidhardt (Hrsg.): Öffentlichkeit, Öffentliche Meinung, soziale Bewegungen, Opladen 1994 (=Sonderheft 34 der Kölner Zeitschrift für Soziologie und Sozialpsychologie), S. 162-190, S. 174. 
ern, werden selten eingesetzt. Bewertungen in dieser Dimension werden zwar konstant verwendet, treten aber im Gesamtprofil der Skandalierten nicht besonders hervor. Eine Ausnahme bilden Einschätzungen des Handelns im Skandalfall, das häufig als verfehlt bezeichnet wird. Damit wird eine fallweise aber keine generelle Inkompetenz konstatiert. Es ist anzunehmen, daß Verweise auf Qualifikation und Expertenstatus vor allem dann wichtige Instrumente zur Stützung der Glaubwürdigkeit sind, wenn Themen angesprochen werden, für die die Stimmen von Fachleuten relevant sind, also wenn technische oder wissenschaftliche Belange in der Diskussion stehen. Im Zusammenhang mit Skandalen wird 'Kompetenz' möglicherweise bei anders gelagerten Normverletzungen als den untersuchten bedeutsamer, etwa bei Umweltskandalen.

\subsubsection{Vertrauenswürdigkeit}

Die Dimension 'Vertrauenswürdigkeit' soll Aufschluß darüber geben, ob und inwieweit ein Ausgangspartner oder Bewertungsobjekt hinsichtlich seiner Aussagen und Handlungen verläßlich ist. Solche Verläßlichkeit findet ihren Ausdruck zunächst in Aussagen über die Ehrlichkeit der betreffenden Person.

Die Variable 'Rechtschaffenheit' sammelt daher alle Hinweise, die etwas darüber aussagen, inwieweit das Verhalten des Bewertungsobjektes in Einklang mit bestehenden rechtlichen Normen ist und wie es um seine Wahrheitsliebe bestellt ist. Positive Nennungen wären hier etwa Verweise auf Einhaltung rechtlicher Normen, auf Gesetzestreue oder Freisprüche durch richterliche Instanzen, Aussagen über die Übereinstimmung von Wort und Tat ${ }^{15}$ auf seiten des Bewertungsobjekts sowie dessen eigene Eingeständnisse von Fehlern,

15 Vgl. Albert Oeckl in einem Vortrag vor dem Bildungsrat der nordrhein-westfälischen Wirtschaft, zitiert nach: Klaas Apitz: Konflikte, Krisen, Katastrophen. Präventivmaßnahmen gegen Imageverlust, Frankfurt a.M./Wiesbaden 1987, S. 23 sowie Katja Götsch: Riskantes Vertrauen. Theoretische und empirische Untersuchung zum Konstrukt Glaubwürdigkeit, Münster/Hamburg 1994, S. 123. 
Schuld, Versäumnissen oder Schwächen. ${ }^{16}$ Negativ zu Buche schlagen Hinweise auf Gesetzesverstöße, Anklagen, Verurteilungen, auch Hinweise auf frühere Verfehlungen oder Verdachtsmomente sowie Aussagen, die das Leugnen oder Zurückhalten von Wissen betreffen. ${ }^{17}$

Neben der Beurteilung des Ausgangspartners wird auch geprüft, ob der Inhalt seiner Aussage eine Beurteilung erfährt, die Zweifel an der Glaubwürdigkeit aufkommen läßt. In Anlehnung an Befunde der forensischen Aussagepsychologie werden zur Erfassung dieses Sachverhalts fünf Variablen konstruiert. Festgehalten wird hier jeweils, was im Rahmen des untersuchten Beitrags von anderen Ausgangspartnern oder vom Vermittler über die Aussage eines Dritten gesagt wird. Unter 'Schilderung von Details' wird erfaßt, ob die Aussage originelle Einzelheiten mitteilt, ob sie konkret, individuell oder anschaulich ist bzw. ob eine oberflächliche, karge, abstrakte Schilderung vorliegt. Die 'Logische Konsistenz' hält fest, ob eine Aussage als schlüssig, in sich logisch, ohne Widersprüche beurteilt wird oder ob fehlende Logik, sprunghafte, unzusammenhängende Wiedergabe oder eine Verwicklung in Widersprüche im Verlauf der Aussage bemängelt werden. Urteile über die 'Konstanz der Aussage' setzen die Aussage zu anderen, früheren Aussagen des gleichen Aussageträgers in Beziehung und betonen Übereinstimmungen oder Widersprüche in zeitlich auseinanderliegenden Befragungen oder Mitteilungen. Hingegen wird mit der Variable 'Übereinstimmung' geprüft, wie es um das Verhältnis der Aussage im Vergleich mit den Aussagen anderer Aussageträger oder mit feststehenden Fakten bestellt ist, das heißt, ob eine Bestätigung durch andere Aussagen vorliegt, ob es Gewährsmänner oder Zeugen für die Darstellung gibt oder ob die Fakten mit der Aussage vereinbar sind bzw. ob die Aussage in Widerspruch zu anderen Versionen steht. Und schließlich werden Urteile über die 'Pauschale Glaubwürdigkeit der Aussage' erfaßt, die ohne nähere Be-

16 Vgl. Daniel G. Linz / Steven Penrod: Increasing Attorney Persuasiveness in the Courtroom, in: Law and Psychology Review 8/1984, S. 1-47, S. 35.

$17 \quad$ Vgl. Götsch (1994), S. $123 f$. 
gründung die Mitteilung als glaubhaft bezeichnen oder anzweifeln.

Im Polaritätenprofil manifestieren sich Charakterisierungen der Ehrlichkeit in den Bedeutungshöfen ehrlich-unehrlich, geradlinig-hinterhältig, redlichbetrügerisch, echt-unecht, rechtmäßig-unrechtmäßig.

Als zweite Komponente der 'Vertrauenswürdigkeit' ist die 'Reinheit der Motive' oder 'Lauterkeit' anzusehen. In diesem Zusammenhang muß das Untersuchungsmaterial daraufhin gesichtet werden, ob sich Hinweise auf die Interessenlage des Ausgangspartners oder Bewertungsobjekts finden lassen. Sofern diesem Parteilichkeit für andere oder Eigennutz (also Parteilichkeit für sich selbst) unterstellt wird, ist von einer Minderung seiner Glaubwürdigkeit auszugehen.

Bedeutsam ist hier auch der Kommunikations-Kontext, das heißt die Kenntnis der Situation, in der die Aussage erfolgte. Daraus kann eine Beeinflussungsabsicht hervorgehen bzw. es können Interessen und Motive des Ausgangspartners zutage treten. ${ }^{18} \mathrm{Im}$ Rahmen der Versuche mit direkter Kommunikation erfolgte eine Manipulation der Kommunikations-Kontexte mit den Konzepten des 'Forewarning' (ausdrücklicher Hinweis auf die Beeinflussungsabsicht der Quelle) und 'Overhearing' (scheinbar zufälliges Belauschen einer Unterhaltung). Eine Übertragung auf Massenkommunikation ist nur bedingt möglich: Zum einen ist die überwiegende Anzahl der Aussagen, die per Massenkommunikation vermittelt werden, "absichtlich" zum Zweck der Veröffentlichung produziert; zum anderen ist fraglich, ob selbst explizite Hinweise auf den Öffentlichkeitscharakter bei den Rezipienten bereits zu Abstrichen hinsichtlich der Glaubwürdigkeit führen. Allenfalls bezüglich spezieller Situationen, wie etwa Wahlkampfreden, scheint dies plausibel.

Obgleich hier also direkte Rückschlüsse hinsichtlich der Glaubwürdigkeit nur mit größter Vorsicht gezogen werden können, wird dennoch der Versuch unternommen, die 'Aussagesituation' zu berücksichtigen. Dabei wird unterschieden zwischen Aussagen, die explizit zum Zweck der Veröffentlichung vor

18 Vgl. Köhnken (1990), S. 121, $163 f$. 
einem größeren Publikum gemacht wurden, halböffentlichen Aussagen, die im Rahmen der Kommunikation innerhalb der eigenen Gruppe getätigt werden und - obwohl kein Veröffentlichungsverbot besteht - nicht primär für Außenstehende gedacht sind, und Gesprächen, die in privatem Rahmen oder im kleinen Kreis stattgefunden haben und die nicht zur Veröffentlichung bestimmt sind. Zu prüfen bleibt, ob insbesondere die letztgenannten - in Verbindung mit anderen Variablen - benutzt werden, um den Eindruck von Glaubwürdigkeit hervorzurufen.

Alle konkreten Aussagen über die Interessenlage des Bewertungsobjekts werden in der Variable 'Lauterkeit' gesammelt. Verknüpft mit der Frage nach der Parteilichkeit ist hier auch die Frage nach der Abhängigkeit bzw. Unabhängigkeit von anderen sowie der Verweis auf Motive, Absichten oder Willensbekundungen. Wie bei der 'Rechtschaffenheit' orientierte sich die Vercodung an exemplarischen Begründungsmustern.

Im Polaritätenprofil findet die 'Lauterkeit' ihren Ausdruck in den Gegensatzpaaren fair-unfair, unparteilich-parteilich, unabhängig-abhängig, unbelastetbelastet, altruistisch-egoistisch, rücksichtsvoll-rücksichtslos. Darüberhinaus gibt es noch eine Reihe von Charakterisierungen, die der Dimension 'Vertrauenswürdigkeit' zuzurechnen sind, ohne daß sie mit einer der beiden Komponenten 'Ehrlichkeit' oder 'Lauterkeit' in direktem Zusammenhang stehen (vertrauenswürdig-nicht vertrauenswürdig, zuverlässig-unzuverlässig, sorgfältig-leichtfertig, verantwortungsbewußt-verantwortungslos, glaubwürdigunglaubwürdig.).

Die Dimension 'Vertrauenswürdigkeit' hat für die Charaktereinschätzung der am Skandal Beteiligten große Bedeutung. In allen untersuchten SkandalFällen vereint sie die meisten Bewertungen auf sich. Je nach Fall und Normverstoß steht dabei eine andere Sub-Dimension im Vordergrund: Im Fall Barschel/Pfeiffer die 'Ehrlichkeit' beider Kontrahenten; im Fall Möllemann die 'Lauterkeit' oder 'Reinheit der Motive'; im Fall Krause beide Sub-Dimensionen gleichermaßen. In allen Fällen überwiegen die negativen Einstufungen bei weitem und sind meist repräsentiert duch die Bedeutungshöfe 'unehrlich', 
'hinterhältig' und 'egoistisch'.

Explizite Bewertungen werden zur Zuschreibung von Vertrauenswürdigkeit am weitaus häufigsten herangezogen. Auch die gesondert erhobenen Variablen 'Rechtschaffenheit' und 'Lauterkeit' sind für die Einschätzung bedeutsam, wobei sich begründete und unbegründete Urteile in der Summe meist die Waage halten.

Die Befunde der Pilotstudie deuten an, daß bei der Berichterstattung über Skandale auch Beurteilungen des Aussageinhalts unter bestimmten Bedingungen als Kriterien für die Glaubwürdigkeit der Beteiligten herangezogen werden. Bei der 'Briefbogen-Affäre' und bei der 'Putzfrauen-Affäre' spielten sie keine Rolle. In diesen Fällen waren auch die Vorwürfe selbst kaum strittig. Zur Diskussion stand lediglich, ob die Handlungen der Skandalierten eine gravierende Normverletzung darstellten. Anders bei der 'Kieler Affäre': Hier war die Einschätzung der Geschehnisse in hohem Maß abhängig davon, ob die Vorwürfe überhaupt als zutreffend erachtet wurden und welcher Version der Vorgänge eher Glauben zu schenken war. Entsprechend stand sowohl für Pfeiffer als auch für Barschel die Übereinstimmung ihrer Aussagen mit den Aussagen Dritter im Blickpunkt. Ihre Aussagen wurden ferner im Hinblick auf ihre allgemeine Glaubwürdigkeit eingeschätzt. Lediglich beim Hauptskandalierten Barschel wurde darüberhinaus auf Widersprüche zu früheren, eigenen Aussagen hingewiesen.

Unter Einbeziehung der übrigen Ergebnisse läßt sich damit festhalten, daß Beurteilungen des Aussageinhalts zwar nicht zum Standardrepertoire bei der Glaubwürdigkeitseinschätzung im Rahmen massenkommunikativ vermittelter Gespräche gehören, daß sie aber im Falle konfligierender Versionen gewisses Gewicht erlangen und für Glaubwürdigkeitseinschätzungen, die auf anderem Weg erfolgen, zumindest unterstützende Funktion haben.

Aus dem Kommunikations-Kontext lassen sich kaum Rückschlüsse auf die Vertrauenswürdigkeit der beteiligten Akteure ziehen. Soweit die Aussagesituation von den Medien transparent gemacht wird, dominieren erwartungsgemäß Medien-Aussagen und die öffentliche Rede. Lediglich im SPIEGEL 
werden Privatgespräche und schriftliche Äußerungen deutlich häufiger ausgewiesen. Es ist allerdings fraglich, ob der SPIEGEL primär daran interessiert ist, die Vertrauenswürdigkeit seiner vermittelten Aussageträger zu stützen oder ob er damit nicht vielmehr seiner eigenen Darstellung Authentizität verleihen will.

\subsubsection{Dynamik / Selbstdarstellung}

Die Dimension 'Dynamik' verweist sowohl auf allgemeine Verhaltensformen wie auf das Sprechverhalten des Aussageträgers. Bei der Inhaltsanalyse von Printmedien stellt sich das Problem, daß das Verhalten des Sprechers nicht der direkten Beobachtung zugänglich ist. Leser sind ebenso wie Forscher darauf angewiesen, ihre Rückschlüsse aus den Schilderungen Dritter zu ziehen. Die Rolle des Betrachters übernimmt hier in aller Regel der Vermittler; die Weitergabe solcher Beobachtungen und Eindrücke erfolgt in interpretierenden Darstellungsformen wie etwa der Reportage.

Aus der Forschung zur verhaltensorientierten Glaubwürdigkeitsbeurteilung sind eine Reihe von Merkmalen bekannt, aus denen Beobachter Rückschlüsse auf die Aufrichtigkeit der Sprecher ziehen. Dagegen liegen keine gesicherten Erkenntnisse darüber vor, ob Auswirkungen auf die Glaubwürdigkeit eines Sprechers gegeben sind, wenn solche Merkmale nur indirekt als Beschreibungen vermittelt werden. Es ist jedoch anzunehmen, daß auch solche Schilderungen für den Prozeß der Eindrucksbildung eine Rolle spielen. Obgleich davon auszugehen ist, daß Beschreibungen des Sprechverhaltens eher selten auftreten, wurden acht Variablen erstellt, die ihre Erfassung ermöglichen. Der Testcharakter dieser Variablen sei ausdrücklich betont.

Die Variablen geben Auskunft darüber, wie sich der Aussageträger im Gespräch oder beim Reden verhält. Das Sprechverhalten läßt sich unterteilen in nonverbales Verhalten (Mimik, Blickkontakt, Gestik und Körperbewegungen) und extralinguistisches Verhalten (Reaktionslatenz und Verzögerungen, 
Sprechstörungen, Stimmhöhe und Stimmqualität). ${ }^{19}$ Bei jeder Variable wird darüberhinaus unterschieden, ob es sich um beobachtbare Verhaltensweisen handelt, das heißt ob jeder potentiell Anwesende sie hätte wahrnehmen können, oder ob die Verhaltensweisen nicht der sinnlichen Wahrnehmung zugänglich sind, das heißt auf der Schilderung von Eindrücken bzw. auf Schlußfolgerungen oder Unterstellungen beruhen oder mit Wertungen verknüpft sind.

Beschreibungen der 'Mimik' verweisen auf Gesichtsbewegungen während des Redevorgangs. Von besonderer Bedeutung im Hinblick auf die Glaubwürdigkeit ist hier vor allem das Lächeln. Auch die Frage, ob 'Blickkontakt' mit den Zuhörern gesucht oder vermieden wird, wird häufig als Indikator für die Redlichkeit des Sprechers herangezogen. Die 'Gestik' betrifft Tätigkeiten, die während des Sprechvorgangs mit den Händen oder Armen ausgeführt werden. Zu erwähnen sind hier insbesondere Verhaltensweisen, die zu einer Minderung der Glaubwürdigkeit beitragen, wie Zittern der Hände, Selbstmanipulationen (z.B. Kratzen, Zupfen an den Lippen) oder Objektmanipulationen (z.B. Spielen mit dem Feuerzeug). Schließlich gilt die Aufmerksamkeit noch sonstigen 'Körperbewegungen' während des Redevorganges (z.B. Zappeln, auf dem Stuhl herumrutschen).

Die Variable 'Reaktionslatenz und Verzögerung' steht für Pausen, die sich bei einem Sprecherwechsel einstellen (= Reaktionslatenz) und Pausen, die im Verlauf einer Aussage auftreten (= Verzögerungen). 'Sprechstörungen' sind Sprechfehler im Verlauf der Aussage (Stottern, Versprecher o.ä.). Die 'Stimmhöhe' bezieht sich auf die Modulation der Stimme (ruhig vs. überschlagend), während die 'Stimmqualität' Aufschluß gibt über stimmliche Eigenschaften (z.B. feste Stimme, nasales Sprechen, Heiserkeit).

Eine weitere Variable hält fest, wie sich der Ausgangspartner seinen Kommunikationspartnern gegenüber verhält. Dafür stehen verschiedene 'Stufen

19 Nach Pretests verworfen wurden Variablen zur Sprechgeschwindigkeit und zur Lautstärke, da hierzu kaum Beispiele in der Berichterstattung auftraten. Hinzu kommt, daß die Forschungsergebnisse eindeutige Interpretationen im Hinblick auf die Glaubwürdigkeit kaum zulassen. 
der Offensive' zur Verfügung, die von Fragen über kooperative Angebote, Aufforderungen, Beschuldigungen bis hin $\mathrm{zu}$ Anklagen und Angriffen reichen. ${ }^{20}$ Hinweise, die für die Vercodung relevant sind, finden sich entweder im Rahmen der Aussage des Ausgangspartners selbst, als sogenannte 'Sprechakte' (z.B.: "Hiermit fordere ich Sie auf, wahrheitsgemäß zu antworten.") oder im Rahmen von Zitateinbindungen, die der Vermittler vornimmt (z.B.: "X beschwert sich über..."). ${ }^{21}$ Die 'Art der Redewiedergabe' gibt Aufschluß darüber, ob dem Aussageträger Gelegenheit zur Selbstdarstellung gegeben wird, indem seine Aussage in direkter Rede vermittelt wird oder ob verschiedene Formen indirekter Rede verwendet werden.

Eine Reihe von Charakterisierungen, die das allgemeine Verhalten des Aussageträgers betreffen, haben ihren Platz im Polaritätenprofil: aktiv-passiv, sanftmütig-aggressiv, stark-schwach, entspannt-angespannt, selbstsicherunsicher, extrovertiert-introvertiert. ${ }^{22}$

Für die 'Art der Redewiedergabe' als Möglichkeit der Selbstdarstellung lassen sich im Vergleich der drei Skandalfälle keine Rückschlüsse ableiten. Die direkte Rede (ausschließlich oder in der Mischung mit anderen Formen) wird zwar bevorzugt, aber in jeweils unterschiedlichem Ausmaß. Einen gleichmäßig herausragenden Bestandteil der Berichterstattung stellt sie lediglich in BILD dar. Der SPIEGEL räumt ihr in zwei von drei Fällen (Möllemann und Barschel) Vorrangstellung ein. FR und WELT verwenden sie in zwei von drei Fällen zurückhaltend: Die FR setzt sie lediglich im Fall Krause verstärkt ein, die WELT im Fall Möllemann. Die FAZ verwendet Formen direkter Rede generell

20 Die Offensivstufen wurden leicht abgewandelt übernommen von: Paul-Ludwig Völzing: Begründen, Erklären, Argumentieren. Modelle und Materialien zu einer Theorie der Metakommunikation, Heidelberg 1979, S. 180f.

21 Zur Rolle von Zitateinbindungen vgl. Simone Christiane Ehmig: Zitateinbindung im SPIEGEL, Mainz 1989 (unveröffentlichte Magisterarbeit).

22 Vergleichbare Gegensatzpaare finden sich auch in der Dimension 'Dynamik' bei Berlo / Lemert / Mertz (1969) und in den Faktoren 'Composure' und 'Extroversion' bei McCroskey / Jenson (1975). 
sparsamer, während die SZ eine nahezu gleichbleibende, gemäßigte Vorliebe dafür entwickelt. Die 'Offensivstufen' werden im vermittelten Gespräch eher sparsam eingesetzt. Verwendung finden bevorzugt Aufforderungen und Vorwürfe, die überwiegend von den Skandalierern ausgehen.

Insgesamt kommt der 'Dynamik' gleichbleibende aber nicht allzu intensive Aufmerksamkeit zu, jedenfalls soweit sie sich in Bewertungen manifestiert. Während Beschreibungen des Sprechverhaltens keine Rolle spielen, werden die Skandalierten in allen Fällen überwiegend positiv eingeschätzt, was ihre Stärke und Aktivität anbelangt. Damit ist 'Dynamik' die einzige Dimension, in der günstige Charakterisierungen für die Skandalierten in der Summe deutlich überwiegen. Andere Personen, soweit sie bewertet werden (Aussageträger ohne Skandal-Rolle oder Skandalierer und Betroffene im Fall Barschel) schneiden diesbezüglich nicht so gut ab: Positive und negative Einschätzungen zur 'Dynamik' halten sich bei ihnen die Waage.

\subsubsection{Soziale Billigung}

Als weiterer Faktor für die Vertrauensbildung ist die Mehrheitsmeinung über den Aussageträger oder das Bewertungsobjekt anzusehen. ${ }^{23}$ Informationen über die 'Soziale Billigung' geben Aufschluß darüber, ob und von welcher Seite einer Quelle Ablehnung entgegenschlägt oder Zustimmung zuteil wird. Im Hinblick auf die 'Ähnlichkeit' ist dabei vor allem von Interesse, wie es um die Haltung der Bezugsgruppe zur Quelle bestellt ist. Die Stellung innerhalb der eigenen Gruppe ist nicht zuletzt bei Skandalen ausschlaggebend. ${ }^{24}$

23 Vgl. Giffin (1967). Dort wird die Mehrheitsmeinung zwar nur auf die Reaktionen eines Präsenzpublikums bezogen, eine Ausweitung auf die vermittelte Zustimmung oder Ablehnung von Teilen der Gesellschaft erscheint aber unproblematisch. Intensivere Beachtung wird dem Phänomen bei Schütz (1972) zuteil.

24 "Entscheidend für den Ausgang eines publizistischen Konflikts und eines Skandals ist nicht die Massivität der Angriffe aus dem gegnerischen Lager. Entscheidend ist das Verhalten der eigenen Seite. Solange die Weggefährten zu dem Angegriffenen stehen, hat er gute Chancen 
Entsprechend umfaßt die Variable 'Akzeptanz' alle Formen der Zustimmung, des Befürwortens, der positiven Verstärkung, Unterstützung oder Rechtfertigung des Bewertungsobjekts. Analog beinhaltet die 'Ablehnung' alle Formen der Zurückweisung, des Angreifens oder Bekämpfens oder der Kritik am Bewertungsobjekt. Dabei ist jeweils von besonderem Interesse, aus welchen Lagern (Ingroup oder Outgroup) die Zustimmung oder Ablehnung kommt. ${ }^{25}$ Damit sind gleichzeitig Rückschlüsse auf die Stellung eines Individuums innerhalb seiner Gruppe möglich. Solche Rückschlüsse lassen sich auch ziehen, wenn man betrachtet, in welcher Beziehung ein Aussageträger zum Skandalierten steht und welche Skandal-Rolle er seinerseits innehat. Geprüft wurde ferner, ob ein Aussageträger soziale Billigung für sich in Anspruch nimmt, indem er, in Form einer Berufung, auf Zustimmung aus verschiedenen gesellschaftlichen Lagern verweist.

Zusätzlichen Aufschluß über die Anerkennung des in Frage stehenden Bewertungsobjekts geben die Gegensatzpaare bejaht-verneint, geachtet-verachtet, unanfechtbar-anfechtbar, integriert-isoliert.

Die 'Soziale Billigung' erweist sich in der Analyse neben der 'Vertrauenswürdigkeit' als zentrale Dimension für die Skandalierten, was die Häufigkeit der Nennungen anbelangt. Der Aspekt der Isolation wird in den Fällen Möllemann und Krause besonders deutlich: Die Ablehnung kommt überwiegend aus den eigenen Reihen und die Ingroup stellt auch einen beträcht-

die Angriffe abzuwehren - unabhängig davon, wie heftig sie sind. (...) Sobald die eigenen Weggefährten freiwillig oder gezwungen auf Distanz gehen, ist die Sache für den Angegriffenen verloren." [Kepplinger (1994), S. 231 f.]

25 Die gruppenspezifische Billigung kann sich auch auf die Wahrnehmung des Kommunikationsinhalts auswirken, wie Kelley und Woodruff zeigen konnten: Eine mittels Tonband präsentierte Rede wurde an mehreren Stellen von Applaus unterbrochen; einem Teil der Versuchspersonen teilte man mit, der Applaus stamme von Mitgliedern der eigenen Bezugsgruppe, den anderen wurden anonyme "Outsider" als Urheber genannt. Die Versuchspersonen, die glaubten, der Beifall komme aus den eigenen Reihen, positionierten das Gesagte näher an den Meinungspositionen der Ingroup, als das objektiv der Fall war. (Vgl. Harold H. Kelley / C. L. Woodruff: Members' Reactions to Apparent Group Approval of a Counternorm Communication, in: Journal of Abnormal and Social Psychology 52/1956, S. 67-74.) 
lichen Anteil an Skandalierern. Im Fall Barschel stellt sich Isolation erst nach dem Rücktritt ein; Ablehnung aus den eigenen Reihen überwiegt lediglich in der Darstellung von FR und SPIEGEL, während sie in WELT und BILD vorwiegend von gegnerischer Seite kommt. Abgesehen von der Haltung der Ingroup ist in allen Fällen massive Ablehnung der Skandalierten zu verzeichnen, die meist einmütig von Vermittlern, Skandalierern und Aussageträgern ohne Skandal-Rolle ausgesprochen wird und in den Bewertungen 'verneint' und 'anfechtbar' zum Ausdruck kommt.

\subsubsection{Sympathie}

Wie die Sichtung der einschlägigen Studien erbrachte, ist der Zusammenhang von Sympathie und Glaubwürdigkeit bzw. eng mit Glaubwürdigkeit verknüpften Dimensionen wie Kompetenz und Vertrauenswürdigkeit nicht immer eindeutig nachzuweisen. Die Pilotstudie sollte Aufschluß darüber geben, inwieweit Bewertungen, die die Dimension 'Sympathie' betreffen, eingesetzt werden. Ersetzen sie andere Komponenten, werden sie ergänzend verwendet oder spielen sie nur eine untergeordnete Rolle?

Der Erhebung von Sympathiezuweisungen dienen die folgenden Bedeutungshöfe: sympathisch-unsympathisch, beliebt-unbeliebt, anziehend-abstoBend, freundlich-unfreundlich, bescheiden-selbstgefällig. Der Sonderfall der physischen Attraktivität wird im Bedeutungshof attraktiv-unattraktiv berücksichtigt, der ausschließlich der (wertenden) Beschreibung des Äußeren vorbehalten ist.

Der Befund ist bei allen untersuchten Skandalfällen eindeutig: Kommt den Dimensionen 'Kompetenz' und 'Dynamik' in Anbetracht der Häufigkeit der einschlägigen Nennungen zumindest noch sekundäre Relevanz zu, so spielen 'Sympathie' und 'Attraktivität' bei der Einschätzung der am Skandal beteiligten Personen keine Rolle - jedenfalls nicht im vermittelten Gespräch über diese Personen. Wie in den Experimenten zur quellenorientierten Glaubwür- 
digkeitsbeurteilung hat die 'Sympathie' allenfalls den Rang eines Ersatzkriteriums. 


\begin{tabular}{|c|c|}
\hline & Variablen zur Realisierung \\
\hline Kompetenz & $\begin{array}{l}\text { * Herkunftskompetenz* Expertenstatus: 'Experte' } \\
\text { * Organisationslegitimation* Expertenstatus: 'Erfahrung' } \\
\begin{array}{l}\text { * Kompetenzrelevanz * Expertenstatus: 'Insider' } \\
\text { * Expertenstatus: 'Ehrungen' } \\
\text { * Expertenstatus: 'Anerkennung' }\end{array} \\
\text { * kompetent-inkompetent * fähig-unfähig * richtig-verfehlt * realistisch- } \\
\text { realitätsfern * geschickt-unbeholfen *klug-dumm *erfahren-unerfahren * } \\
\text { planend-planlos }\end{array}$ \\
\hline $\begin{array}{l}\text { Vertrauens- } \\
\text { würdigkeit }\end{array}$ & $\begin{array}{l}\text { * Detailschilderung } \\
\text { * Logische Konsistenz } \\
\text { * Konstanz der Aussage } \\
\text { * Übereinstimmung der Aussage } \\
\text { * Pauschale Glaubwürdigkeit } \\
\text { * Aussagesituation } \\
\text { * ehrlich-unehrlich * geradlinig-hinterhältig * redlich-betrügerisch } \\
\text { * echt-unecht * rechtmäßig-unrechtmäßig * fair-unfair * unparteilich- } \\
\text { parteilich *unabhängig-abhängig *unbelastet-belastet * altruistisch- } \\
\text { egoistisch *rücksichtsvoll-rücksichtslos * vertrauenswürdig-nicht ver- } \\
\text { trauenswürdig* zuverlässig-unzuverlässig * sorgfältig-leichtfertig * ver- } \\
\text { antwortungsbewußt-verantwortungslos *glaubwürdig-unglaubwürdig }\end{array}$ \\
\hline Dynamik & $\begin{array}{l}\text { * Mimik * Blickkontakt* Gestik* Körperbewegungen } \\
\text { * Reaktionslatenz * Sprechstörungen * Stimmhöhe * Stimmqualität } \\
\text { * Art der Redewiedergabe * Offensivstufen der Aussage } \\
\text { *aktiv-passiV*sanftmütig-aggressiV *stark-schwach * entspannt-angespannt } \\
\text { * selbstsicher-unsicher * extrovertiert-introvertiert }\end{array}$ \\
\hline $\begin{array}{l}\text { Soziale } \\
\text { Billigung }\end{array}$ & $\begin{array}{l}\text { * Berufung auf andere* Akzeptanz/Soziale Billigung } \\
\text { * Beziehung zum Skandalierten* Ablehnung/Soziale Mißbilligung } \\
\text { * bejaht-verneint * geachtet-verachtet * unanfechtbar-anfechtbar } \\
\text { * integriert-isoliert }\end{array}$ \\
\hline Sympathie & $\begin{array}{l}\text { * sympathisch-unsympathisch * beliebt-unbeliebt *anziehend-abstoßend * } \\
\text { freundlich-unfreundlich * bescheiden-selbstgefällig } \\
\text { * attraktiv-unattraktiv }\end{array}$ \\
\hline
\end{tabular}

Abb. 2: Glaubwürdigkeits-Dimensionen und Variablen im Überblick 


\subsection{Die Berichterstattung über Skandale: Zentrale Ergebnisse}

Die Glaubwürdigkeit hat im vermittelten Gespräch über politische Skandale durchweg einen hohen Stellenwert, wobei (außer im Fall Barschel) allein die Glaubwürdigkeit der Skandalierten, nicht die ihrer Angreifer auf dem Prüfstand steht und - mehr oder weniger gezielt - demontiert wird. In allen Glaubwürdigkeits-Dimensionen wird für die Einschätzung überwiegend auf wertende Charakterisierungen zurückgegriffen. Dabei handelt es sich in der Mehrzahl um diffuse, nicht näher begründete Zuschreibungen.

Zumindest in den Fällen Möllemann und Krause finden sich aber Hinweise, die vermuten lassen, daß Begründungen für die Werturteile (etwa in Form von Beispielen oder nachvollziehbaren, gegebenenfalls nachprüfbaren Konkretisierungen) gezielt zur Untermauerung des eigenen Standpunkts eingesetzt werden. Verwendet werden Begründungen vor allem von Vermittlern. Dort, wo die Vermittlungsstrategie insgesamt eher eine Gegnerschaft zum Skandalierten erkennen läßt, werden eher negative Einschätzungen begründet (SZ im Fall Möllemann; WELT und SPIEGEL im Fall Krause); dort, wo die Vermittler den Skandalierten nicht ablehnen, werden eher positive Charakterisierungen fundiert (FAZ im Fall Krause). Diese Beobachtungen treffen allerdings nicht für alle Medien zu und im Fall Barschel war ein derartiges Muster gar nicht erkennbar. Dennoch liefern die Befunde Anregungen für weitere Untersuchungen, in denen mit einem verfeinerten Instrumentarium geprüft werden sollte, unter welchen Bedingungen und auf welche Weise solche Begründungen geliefert werden.

Gleiches gilt für die zeitliche Generalisierung von Bewertungen. Zwar treten Einschränkungen oder Verallgemeinerungen selten auf, aber wenn, werden sie von den Vermittlern im Einklang mit der jeweiligen publizistischen Tendenz verwendet: die Charakterisierung verweist entweder auf einen einmaligen, untypischen Sonderfall (WELT und BILD im Fall Möllemann) oder wird öfter als häufig auftretendes, konstantes Merkmal präsentiert (FR und SZ im Fall Möllemann; WELT, FR und BILD im Fall Krause). 
Bei der Einordnung der Befunde zur Glaubwürdigkeit ist in Betracht zu ziehen, daß die Trennung in unterschiedliche Dimensionen ein Konstrukt darstellt, das Forschern ermöglicht, einzelne Merkmale einer Prüfung zu unterziehen. Eine Reihe von Studien hat aber gezeigt, daß in der Wahrnehmung der Rezipienten diese Trennung verschwindet; die Dimensionen sind nicht voneinander unabhängig, sondern beeinflussen sich gegenseitig: Der Eindruck von 'Kompetenz' etwa kann die 'Vertrauenswürdigkeit' erhöhen. Eigenheiten im Sprechverhalten prägen nicht nur die Wahrnehmung der 'Dynamik' sondern zeigen auch Wirkung bei der Einschätzung von 'Kompetenz', 'Vertrauenswürdigkeit' und 'Sympathie'. 'Attraktivität' erhöht die 'Sympathie', führt aber in der Regel nicht dazu, daß eine Quelle für kompetenter oder vertrauenswürdiger gehalten wird. Tatsächliche oder auch nur angenommene 'Ähnlichkeiten' zwischen Quelle und Rezipient tragen dazu bei, daß eine Quelle als sympathischer eingestuft wird; umgekehrt wird einer sympathischen Quelle oft größere 'Ähnlichkeit' zugeschrieben. Unter bestimmten Bedingungen (etwa wenn das Thema für die Gruppe relevant ist) wirkt sich 'Ähnlichkeit' auch auf die Einschätzung von 'Kompetenz' und 'Vertrauenswürdigkeit' aus.

Ob die Akzentuierung bestimmter Merkmale der Quelle die Wahrnehmung der Glaubwürdigkeit auf Rezipientenseite stützt oder mindert, hängt schließlich nicht zuletzt vom Rezipienten selbst ab: Persönlichkeitsspezifische Eigenschaften spielen dabei ebenso eine Rolle wie die Relevanz des Themas für den Rezipienten und sein Vorwissen ('Involvement'). Zu berücksichtigen sind ferner der Zeitpunkt der Information über die Quelle sowie Aufbau, Stil und Inhalt der Mitteilung.

Zieht man all diese Einflußfaktoren in Betracht, so verbietet sich ein direkter Schluß auf die Wirkung, welche die Berichterstattung hervorruft. Allerdings ist wohl davon auszugehen, daß die Glaubwürdigkeitsdefizite, die den Skandalierten im vermittelten Gespräch bescheinigt werden, auf deren Bild bei den Rezipienten nicht ohne Einfluß bleiben, zumal ihnen zur Orientierung lediglich dieses durch die Medien vermittelte Gespräch zur Verfügung steht.

Abgesehen von den Erkenntnissen hinsichtlich der Glaubwürdigkeits- 
zuschreibung via Massenmedien lieferte die Analyse Hinweise auf Muster bei der Skandalberichterstattung, die anhand weiterer Fälle hinterfragt werden sollten:

* Skandalfälle werden nicht generell hervorgehoben präsentiert. So erhält der gleiche Skandalfall nicht in allen Medien die gleiche Aufmerksamkeit. Hinsichtlich der zentralen Beachtungskriterien (Plazierung auf der Titelseite, Größe der Überschriften) ergeben sich zwischen den einzelnen Medien Unterschiede, die darauf hindeuten, daß die Redaktionen Normverstöße jeweils unterschiedlich gewichten oder Hervorhebungen von ihrer Haltung zu den beteiligten Personen abhängig machen.

* Hervorhebungen bei der Präsentation lassen noch keine Schlußfolgerungen darauf zu, ob die Medien journalistischen oder publizistischen Vermittlungsstrategien zugeneigt sind.

* Etikettierungen als 'Skandal' oder 'Affäre' gehen in erster Linie von den Vermittlern aus. Allgemeine Empörungsäußerungen finden sich dagegen sowohl bei Vermittlern als auch bei gesellschaftlichen Aussageträgern.

* Aussageträgern, die in den Skandalverlauf nicht unmittelbar involviert sind, wird in der Berichterstattung große Bedeutung beigemessen, die sich in Umfangs- und Bewertungs-Anteilen manifestiert. Größtenteils gehen diese Sprecher ohne Skandal-Rolle hinsichtlich ihrer Themen und Bewertungen mit den Vermittler-Aussagen konform. Damit liegt der Verdacht nahe, daß sie von den Vermittlern ausgewählt und instrumentalisiert werden, um "publizistische Feldstärken" (Noelle-Neumann) zu erzeugen. Bei der Selektion scheint das Prinzip "Masse statt Klasse" zu gelten, denn diese Partner werden nicht mit Glaubwürdigkeitsmerkmalen ausgestattet. Selbst einfache Kompetenzhinweise fehlen häufig; sie werden nicht ausreichend vorgestellt, sondern bleiben anonym. 
Neben diesen Gemeinsamkeiten ergeben sich bei der Analyse der SkandalFälle Unterschiede zwischen der 'Briefbogen'- und der 'Putzfrauen-Affäre' einerseits sowie der 'Kieler Affäre' andererseits:

* Für die Fälle Möllemann und Krause bestätigt sich, daß Skandale tendenziell einseitige Konflikte sind. ${ }^{26}$ Die Einseitigkeit manifestiert sich nicht unbedingt in den Sprechmöglichkeiten der beteiligten Aussageträger - die Skandalierten kommen eher häufiger und umfangreicher zu Wort als die Skandalierer - sondern eher in den Gesprächsinhalten und in der Objektstruktur: Zum einen sind die Skandalierten thematisch stark in eine Verteidigungshaltung gedrängt, zum anderen sind sie auf die Rolle als Bewertungsobjekt festgelegt. Sie - und nur sie - werden von allen Seiten und besonders stark von den Vermittlern mit Attributionen versehen, kommen selbst aber allenfalls mit Eigencharakterisierungen zu Wort und können ihre Angreifer nicht bewerten. Im Fall Barschel verstärken sich in den tendenziell "linken" Medien die Gesprächsanteile der Skandalierer. Sowohl Skandalierer als auch Skandalierte sind Objekte der Bewertung ebenso wie die Partner ohne Skandal-Rolle, auf die sogar die Mehrzahl der Kennungen entfällt. Die letzteren sind dabei stärker den Bewertungen durch die Vermittler unterworfen als Skandalierer und Skandalierte. Eine ausschließliche Konzentration auf die Skandalierten ist damit in diesem Fall nicht gegeben.

* Die ausgeprägt negative Bewertungstendenz, die in den Fällen Möllemann und Krause vorherrschend war, wird im Fall Barschel für die SkandaliertenSeite nicht aufrechterhalten. Insgesamt wird die Skandalierer-Seite weitaus negativer eingestuft. Eine mit den Fällen Möllemann und Krause vergleichbare Intensität abwertender Stellungnahmen für die Skandalierten-

26 Vgl. Kepplinger / Hartung (1993), S. 6. 
Seite stellt sich erst nach Barschels Rücktritt ein und dauert bis zu seinem Tod an. Dies gilt allerdings nur für die Tageszeitungen. Im SPIEGEL ist Barschel von Anfang an ausgeprägt negativen Attributionen ausgesetzt, während die Skandalierer-Seite - vor allem in der ersten Phase - besser eingestuft wird.

* Auch im Hinblick auf die Themensetzung zeigen sich Unterschiede. Zentrales Thema in allen Fällen und für alle Gesprächsteilnehmer ist das Verhalten der Skandalierten. Diese bringen zusätzlich Elemente der Verteidigung zur Sprache, während die Skandalierer Empörung und Normverletzung betonen. Dieser Akzentuierung schließen sich Vermittler und Partner ohne Rolle in den Fällen Krause und Möllemann an, nicht aber im Fall Barschel. Dort sprechen beide - zumindest in den Tageszeitungen statt dessen mehr über das Verhalten des Skandalierers, Aufklärungsmaßnahmen, Schadensbereinigung und öffentliche Reaktionen. Lediglich die Vermittler des SPIEGEL weisen nachdrücklich auf Normverletzungen hin.

Zieht man zusätzlich in Betracht, daß im Fall Barschel berichtende Darstellungsformen einen höheren Stellenwert haben und Etikettierungen als 'Skandal' oder 'Affäre' sparsamer verwendet werden, so ergibt sich ein paradoxer Befund: Der "größte bekanntgewordene Wahlskandal der $\mathrm{BRD}^{27}$ wird in der Berichterstattung nicht wie ein Skandal - oder besser: nicht wie andere Skandale - behandelt, sondern eher wie ein gewöhnlicher Konflikt, bei dem sich zwei Lager annähernd gleichberechtigt gegenüberstehen. Nur im SPIEGEL sind die Berichterstattungsmuster (abgesehen von der Objektstruktur) mit denen der anderen untersuchten Fälle vergleichbar. In allen anderen Medien sind die Gemeinsamkeiten der Fälle Möllemann und Krause hinsichtlich

27 Rolf Winiarski: Das Gesetz der Gesetzlosen. Zur Dynamik des Skandals am Beispiel der Kieler CDU-Affäre, in: Helmut Moser (Hrsg.): L' Eclat c'est moi. Zur Faszination unserer Skandale, Weinheim 1989, S. 52-65, S. 53. 
Stimmenvielfalt, Themenstruktur, Konzentration auf den Skandalierten als Bewertungsobjekt und Richtung der Bewertung ebenso augenfällig wie die diesbezüglichen Unterschiede zum Fall Barschel. Es wäre anhand weiterer Skandalfälle zu prüfen, ob die 'Kieler Affäre' eine einmalige Ausnahmeerscheinung darstellt oder ob solche Variationen im vermittelten Gespräch häufiger auftreten und es eine spezifische "Skandalberichterstattung" somit nicht gibt.

Bei der Berichterstattung über Sachverhalte aller Art lassen sich generell idealtypisch zwei Vermittlungsstrategien unterscheiden: die journalistische und die publizistische. Der 'Journalist' ist unabhängig von einzelnen Interessen und erfüllt entsprechend die nötigen Selektions- und Transformationsleistungen so neutral wie möglich. Er ist bemüht, alle Beteiligten im Rahmen referierender Berichte zu Wort kommen zu lassen und ihre Aussagen mitteilungsadäquat zu vermitteln. Die Vermittlung seiner eigenen Position geschieht zusätzlich und unter Einhaltung des Postulats der Trennung von Nachricht und Kommentar. Der 'Publizist' macht - seine eigenen oder fremde - Interessen zum Maßstab der Vermittlung. Seine Parteilichkeit manifestiert sich im Verschweigen von Positionen oder darin, daß einzelnen Gesprächsteilnehmern hinsichtlich Plazierung und Umfang ihrer Aussagen Vor- oder Nachteile entstehen sowie darin, daß er selbst mit Wertungen in das vermittelte Gespräch eingreift.

Bei allen untersuchten Skandalfällen treten solche publizistischen Eingriffe zutage - je nach Fall und Medium in unterschiedlicher Intensität. Sie richten sich nicht immer gegen den Skandalierten, sondern können auch dessen Unterstützung dienen. Gegnerschaft kommt zum Ausdruck, indem der Skandalruf besonders lautstark ertönt, Empörung und Normverletzung - sowohl von Vermittlern als auch von gesellschaftlichen Aussageträgern - hervorgehoben werden und die Skandalierten wenig Raum zur Darstellung ihrer Person und Position haben, während die Mehrzahl der vermittelten Aussageträger sowie die Publizisten selbst ausgeprägt negative Urteile - hauptsächlich im Hinblick auf die Glaubwürdigkeit der Skandalierten - abgeben.

Im SPIEGEL ist das die gängige Vorgehensweise: Alle drei Fälle werden 
auf diese Weise präsentiert. Der Fall Möllemann tritt dabei besonders kraß hervor: Geliefert wird im wesentlichen ein Monolog, bei dem 80 Prozent des Umfanges und über 70 Prozent der Bewertungen auf den Publizisten entfallen. Auch die FR erweist sich in allen Fällen als Gegner der Skandalierten. Die SZ ergreift Partei gegen Möllemann und - weniger ausgeprägt - gegen Krause, der auch in WELT und BILD verstärkt angegriffen wird.

Dort, wo Medien als Unterstützer der Skandalierten auszumachen sind (FAZ und BILD in den Fällen Möllemann und Barschel; WELT im Fall Barschel) geben sie diesen mehr Sprechraum und mehr Gelegenheit zu günstiger Selbstdarstellung, bewerten sie meist positiver oder zumindest weniger negativ in den zentralen Glaubwürdigkeits-Dimensionen und beschneiden die Möglichkeiten der Skandalierer, Bewertungen abzugeben. Sie gehen aber in keinem Fall so weit, sich offensichtlich auf die Seite der Skandalierer zu stellen, indem sie diese verteidigen oder die Etikettierungen ablehnen oder relativieren. Die Hilfestellung kommt eher indirekt zum Ausdruck, dadurch daß Skandalrufe und Normverletzungen seltener ausgesprochen werden.

Gleichgültig für welche Seite sie Partei ergreifen - wenn die selbstvermittelnden Anteile der Publizisten steigen, scheinen sie zudem grundlegende journalistische Pflichten zu vernachlässigen: In diesem Fall werden Aussageträger häufiger nicht vorgestellt sondern bleiben anonym, und auch die Aussagesituation wird öfter verschwiegen. Eine bessere Transparenz und eine bessere Orientierung für die Leser ist gewährleistet, wenn näherungsweise eine journalistische Vermittlungsstrategie verfolgt wird. Dies ist der Fall in der WELT bei der 'Briefbogen-Affäre', in der FAZ bei der 'Putzfrauen-Affäre' und in der SZ bei der 'Kieler Affäre'.

\subsection{Anwendungsmöglichkeiten}

Die Glaubwürdigkeit von Personen im Prozeß der Massenkommunikation wird 
für den Rezipienten immer dann wichtig, wenn zwei Bedingungen erfüllt sind: Zum einen schenkt er den Quellen dann mehr Aufmerksamkeit, wenn er auf einem Gebiet nicht über genügend Wissen verfügt, um sich eigenständig ein Urteil bilden zu können. Zum anderen gewinnt diese Art der Orientierung immer dann an Bedeutung, wenn bezüglich eines Themas oder Sachverhalts in der Gesellschaft keine Einstimmigkeit herrscht, immer dann also, wenn sich zwischen verschiedenen gesellschaftlichen Gruppen Diskussionen oder Konflikte entzünden.

Allen voran sind hier die thematischen "Dauerbrenner" Atomenergie und Gentechnologie zu nennen, die immer dann verstärkt ins Bewußtsein der Öffentlichkeit und in die Diskussion geraten, wenn sich Probleme einstellen (z.B. Störfälle, Atommülltransporte) oder wenn sich neue Anwendungsmöglichkeiten ergeben (z.B. Klonen von Säugetieren, gentechnisch veränderte Organismen). Aber die beiden Bedingungen - Laienstatus des Rezipienten und kontroverse Diskussion in der Gesellschaft - sind nicht nur für Themen aus den Bereichen Wissenschaft und Technik erfüllt. Auch in den Bereichen Politik und Wirtschaft gehören Konfliktthemen und -situationen zum Alltag, in jüngster Zeit etwa die Debatten um die europäische Währungsunion und die Steuerreform. Zu fragen wäre schließlich auch, ob und wie die Glaubwürdigkeit von Politikern in Wahlkampfzeiten in den Blickpunkt gerät.

Aus kommunikationswissenschaftlicher Sicht interessiert vor allem die Frage, ob und wie die wahrgenommenen Glaubwürdigkeitsmerkmale von Quellen durch die Darstellung der Medien beeinflußt werden. Spätestens wenn Einseitigkeiten in der Berichterstattung den Rezipienten bewußt werden, ist auch die Glaubwürdigkeit der Medien in Gefahr, da diese sich an ähnlichen Kriterien mißt wie die Glaubwürdigkeit der Quellen: Der 'Vertrauenswürdigkeit' der Quelle - konkretisiert durch Ehrlichkeit und Abwesenheit von Eigeninteressen - entspricht auf Medienseite die Erfüllung der Forderung nach Fairneß, Unparteilichkeit und Objektivität. 'Kompetenz' heißt für die Quelle Fachkompetenz bezüglich eines Themas. Solche Fachkompetenz wird von universellen Medien nicht verlangt; sie müssen jedoch Vermittlungskompetenz 
aufweisen, die sich im sachgerechten Umgang mit Quellen und deren Mitteilungen manifestiert. Dazu gehört die Beantwortung der W-Fragen ebenso wie eine angemessene Selektion und Transformation. Die 'Dynamik' der Quelle - also ihr Auftreten und ihr Sprechverhalten - verlagert sich bei den Medien auf formale Elemente der Aufmachung und Präsentation. Welchen Einfluß etwa das Layout oder die Größe der Überschriften auf die wahrgenommene Glaubwürdigkeit hat ist ebenso ungeklärt wie die Frage, nach der Auswirkung unterschiedlicher Sympathieeinstufungen. Auch daß 'Ähnlichkeit' bezüglich der Werthaltungen von Medien und Rezipienten für die Glaubwürdigkeitseinschätzung eine Rolle spielen, oder daß 'Soziale Billigung' (wie sie etwa in der Bezeichnung "Prestigemedien" zum Ausdruck kommt) von Belang ist, kann vorerst nur vermutet werden. Anders als bei der Untersuchung der Glaubwürdigkeit von Quellen, bei der konkrete Merkmale geprüft wurden, beschränkte sich die Forschung bei der Glaubwürdigkeit von Medien lange Zeit auf die eindimensionale Frage nach der Glaubhaftigkeit konfligierender Versionen in Fernsehen, Hörfunk und Zeitung oder auf die Vorlage von Semantischen Differentialen, die aus der personenbezogenen Forschung entlehnt wurden. Erst in jüngerer Zeit werden medienspezifische Gegebenheiten berücksichtigt; die Frage nach konkreten Einzelmedien oder Sendungen bleibt jedoch die Ausnahme. ${ }^{28}$

Defizite bei der Untersuchung der Glaubwürdigkeit von Medien entstehen schließlich nicht zuletzt durch die Vernachlässigung der inhaltlichen und stilistischen Elemente. Woran liegt es, daß "Infofiction"29 erst dann unglaub-

28 Daß konkretere Fragen in diesem Bereich auch konkrete Ergebnisse liefern belegt eine Forsa-Umfrage: "Die 'Schicksals-Talkshows' im deutschen Fernsehen genießen trotz hoher Einschaltquoten kaum Vertrauen bei den Zuschauern. Nur zehn Prozent von 1002 Befragten glauben einer Forsa-Umfrage zufolge, daß alles oder das meiste, was ihnen die Gäste von Fliege, Meiser, Kerner und Co. an bewegenden Erlebnissen präsentieren, wahr ist. Dagegen waren 25 Prozent sicher, daß alles oder das meiste an den oft tragischen Bekenntnissen gefälscht ist." (o.V.: Kaum Glauben an TV-Schicksale, in: Kölner Stadt-Anzeiger vom 5. Mai 1997.) 'Die Zeit' vom 02.02.1996) im Zusammenhang mit den Fälschungen von Fernseh-Reportagen. 
würdig wird, wenn die Fiktion enttarnt wird? Diese Frage bedarf ebenso einer systematischen Klärung wie die, welchen Einfluß das "Infotainment" auf die Glaubwürdigkeit der Medien hat. ${ }^{30}$

Die Bedeutung formaler Elemente wurde bislang eher von den Werbetreibenden als von den Forschern erkannt: Anleihen bei der redaktionellen Gestaltung äußern sich in "Infomercials", die etwa im Gewand von Nachrichtensendungen daherkommen. ${ }^{31}$ Auf diese Weise mag zwar die Glaubwürdigkeit der Werbung erhöht werden, ${ }^{32}$ die der Medien kann aber in Mitleidenschaft gezogen werden. Dies wurde 1995 am Fallbeispiel Ulrich Wikert deutlich, dessen Teilnahme an einem solchen "Infomercial" harsch kritisiert wurde.

Die Betrachtung dieser drei Gebilde (Infofiction, Infotainment und Infomercial) legt die Vermutung nahe, daß die Glaubwürdigkeit der Information und damit die ihrer Vermittler - immer dann leidet, wenn sie sich mit anderen Formen paart und Info-Bastarde zeugt. Ob und unter welchen Bedingungen sich diese Vermutung bestätigt, bedarf einer intensiven Prüfung. Um näheren Aufschluß über die Faktoren zu erlangen, die zur Glaubwürdigkeit von Medien beitragen, bietet sich an, von den eingefahrenen Forschungspfaden erschienenen Buch "Breaking the News". Er warnt darin vor Gefahren für die Demokratie, wenn sich Journalisten von ihrem "eigentlichen Informationsauftrag" entfernen. (Zitiert nach: o. V. (dpa): Gefahren eines "Infotainment". James Fallows löst heftige Debatten über Glaubwürdigkeit des US-Journalismus aus, in: Kölner Stadt-Anzeiger vom 05.02.1996.)

Der Begriff "Infomercial" - eine Kombination von 'Information' und 'Commercial' bezieht sich in erster Linie auf Fernsehen und Hörfunk. Derlei Werbeformen sind aber auch im Printbereich gebräuchlich: Mit sogenannten "Feature Ads" wird ausdrücklich darauf spekuliert, an der Glaubwürdigkeit des redaktionellen Umfelds zu partizipieren, indem dessen Gestaltungsprinzipien übernommen werden. (Vgl. dazu: Glen T. Cameron / John Eric Haley: Feature Advertising: Policies and Attitudes in Print Media, in: Journal of Advertising 21/1992, S. 47-55.) lediglich 14 Prozent der Befragten Werbespots mit Fernsehjournalisten überzeugender, 78 Prozent halten sie für nicht glaubwürdiger als andere. (Zitiert nach: o.V.: Werbeauftritte überzeugen nicht, in: Journalist 2/1996, S. 10.) 
abzuweichen und neben Befragungen verstärkt experimentelle Untersuchungsdesigns anzuwenden. 


\section{Literaturverzeichnis}

Aaker, David A. / Phillip K. Brown: Evaluating Vehicle Source Effects, in: Journal of Advertising Research 12/1972, S. 11-16.

Abel, John D. / Michael O. Wirth: Newspaper vs. TV Credibility for Local News, in: Journalism Quarterly 54/1977, S. 371-375.

Adams, John B.: Effects of Reference Group And Status on Opinion Change, in: Journalism Quarterly 37/1960, S. 408-412.

Adams, John B.: The Relative Credibility of 20 Unnamed News Sources, in: Journalism Quarterly 39/1962, S. 79-82

Adams, John B.: Unnamed Sources and the News: A Follow-Up Study, in: Journalism Quarterly 41/1964, S. 262-264.

Addington, David W.: The Effect of Vocal Variations on Ratings of Source Credibility, in: Speech Monographs 38/1971, S. 242-247.

Adorno, Theodor W. et al.: The Authoritarian Personality, New York 1950.

Ajzen, Icek: Effects of Information on Interpersonal Attraction: Similarity versus Affective Value, in: Journal of Personality and Social Psychology 29/1974, S. 374-380.

Alemann, Ulrich von:Politische Moral und politische Kultur in der Bundesrepublik - Vergiften oder reinigen Skandale die Politik? in: Gewerkschaftliche Monatshefte 5/1985, S. 258-269.

Alemann, Ulrich von: Technik und Interesse. Anmerkungen zu Grundbegriffen und Folgerungen für Glaubwürdigkeit von Experten und Gutachten, in: Walter Ch. Zimmerli / Hansjörg Sinn: Die Glaubwürdigkeit technisch-wissenschaftlicher Experten, Düsseldorf 1990, S. 49-66.

Allen, Mike / James B. Stiff: Testing Three Models for the Sleeper Effect, in: Western Journal of Speech Communication 53/1989, S. 411-426.

Al-Makaty, Safran S. / Douglas A. Boyd / G. Norman Van Tubergen: Source Credibility during the Gulf War: A Q-Study of Rural and Urban Saudi Arabian Citizens, in: Journalism Quarterly 71/1994, S. 55-63.

Andersen, Kenneth / Theodore Clevenger, Jr.: A Summary of Experimental Research in Ethos, in: Speech Monographs 30/1963, S. 59-78.

Anderson, Lynn R.: Belief Defense Produced by Derogation of Message Source, in: Journal of Experimental Social Psychology 3/1967, S. 349-360. 
Andreoli, Virginia / Stephen Worchel: Effects of Media, Communicator, and Message Position on Attitude Change, in: Public Opinion Quarterly 42/1978, S. 59-70.

Andsager, Julie L.: Perceptions of Credibility of Male and Female Syndicated Political Columnists, in: Journalism Quarterly 67/1990, S. 485-491.

Annis, Albert D. / Norman C. Meier: The Induction of Opinion through Suffestion by Means of "Planted Content", in: Journal of Social Psychology 5/1934, S. 65-81.

Apitz, Klaas: Konflikte, Krisen, Katastrophen. Präventivmaßnahmen gegen Imageverlust, Frankfurt a.M. / Wiesbaden 1987.

Applbaum, Ronald L. / Karl W. E. Anatol: The Factor Structure of Source Credibility as a Function of the Speaking Situation, in: Speech Monographs 39/1972, S. 216-222.

Applbaum, Ronald L. / Karl W. E. Anatol: Dimensions of Source Credibility: A Test for Reproducibility, in: Speech Monographs 40/1973, S. 231-237.

Applbaum, Ronald L. / Karl W. E. Anatol: A Rejoinder, in: Speech Monographs 41/1974, S. 295298.

Aristoteles: Rhetorik, Paderborn 1959 [übersetzt von Paul Gohlke].

Arnett, Claude E. / Helen H. Davidson / Hallett N. Lewis: Prestige as a Factor in Attitude Changes, in: Sociology and Social Research 16/1931, S. 49-55.

Arnold, William E. / James C. McCroskey: The Credibility of Reluctant Testimony, in: Central States Speech Journal 18/1967, S. 97-103.

Arntzen, Friedrich: Die Situation der forensischen Aussagepsychologie in der Bundesrepublik Deutschland, in: Trankell (1982), S. 107-120.

Arntzen, Friedrich: Psychologie der Zeugenaussage. System der Glaubwürdigkeitsmerkmale, München ${ }^{3} 1993$.

Aronson, Elliot / Burton W. Golden: The Effect of Relevant and Irrelevant Aspects of Communicator Credibility on Opinion Change, in: Journal of Personality 30/1962, S. 135-146.

Aronson, Elliot / Judith A. Turner / J. Merrill Carlsmith: Communicator Credibility and Communication Discrepancy as Determinants of Opinion Change, in: Journal of Abnormal and Social Psychology 67/1963, S. 31-36.

Asch, Solomon E.: The Doctrine of Suggestion, Prestige, and Imitation in Social Psychology, in: Psychological Review 55/1948, S. 250-276.

Atwood, L. Erwin: The Effects of Incongruity Between Source and Message Credibility, in: Journalism Quarterly 43/1966, S. 90-94.

Aune, R. Kelly / Toshiyuki Kikuchi: Effects of Language Intensity Similarity on Perceptions of Credibility, Relational Attributions, and Persuasion, in: Journal of Language and Social Psychology 12/1993, S. 224-238.

Austin, Erica Weintraub / Qingwen Dong: Source v. Content Effects on Judgments of News Believability, in: Journalism Quarterly 71/1994, S. 973-983. 
Backman, Carl W. / Paul F. Secord (Hg.): Problems in Social Psychology. Selected Readings, New York u.a. 1966

Baesler, E. James / Judee K. Burgoon: The Temporal Effects of Story and Statistical Evidence on Belief Change, in: Communication Research 21/1994, S. 582-602.

Baggeley, Jon: Psychology of the TV Image, Westmead 1980.

Baker, Eldon E.: The Immediate Effects of Perceived Speaker Disorganization on Speaker Credibility and Audience Attitude Change in Persuasive Speaking, in: Western Speech 29/1965, S. 148-161.

Baker, Michael J. / Gilbert A. Churchill, Jr.: The Impact of Physically Attractive Models on Advertising Research, in: Journal of Marketing Research 14/1977, S. 538-555.

Bannister, Brendan D.: Performance Outcome Feedback and Attributional Feedback: Interactive Effects on Recipient Responses, in: Journal of Applied Psychology 71/1986, S. 203-210.

Barry, Anita K.: Narrative Style and Witness Testimony, in: Journal of Narrative and Life History 1/1991, S. 281-293.

Barry, Thomas E.: Comparative Advertising: What Have We Learned in Two Decades? In: Journal of Advertising Research 33/1993, S. 19-29.

Basehart, John R. / Robert N. Bostrom: Credibility of Source and of Self in Attitude Change, in: Journalism Quarterly 49/1972, S. 742-745.

Bauchner, Joyce E. / David R. Brandt / Gerald R. Miller: The Truth/Deception Attribution: Effects of Varying Levels of Information Availability, in: Brent D. Ruben (Hrsg.): Communication Yearbook I, New Brunswick, N.J. 1977, S. 229-243.

Baudhuin, E. Scott / Margaret Kis Davis: Scales for the Measurement of Ethos: Another Attempt, in: Speech Monographs 39/1972, S. 296-301.

Baxter, Leslie A. / John R. Bittner: High School and College Student Perceptions of Media Credibility, in: Journalism Quarterly 51/1974, S. 517-520.

Beach, Lee Roy et al.: Information Relevance, Content and Source Credibility in the Revision of Opinions, in: Organizational Behavior and Human Performance 21/1978, S. 1-16.

Becker, Lee B. / Raymond A. Martino / Wayne M. Towers: Media Advertising Credibility, in: Journalism Quarterly 53/1976, S. 216-222.

Bender, Hans-Udo: Merkmalskombinationen in Aussagen. Theorie und Empirie zum Beweiswert beim Zusammentreffen von Glaubwürdigkeitskriterien, Tübingen 1987.

Bentele, Günter: Der Faktor Glaubwürdigkeit. Forschungsergebnisse und Fragen für die Sozialisationsperspektive, in: Publizistik 33/1988, S, 406-426.

Bentele, Günter: Objektivitätsanspruch und Glaubwürdigkeit, in: Otfried Jarren (Hrsg.): Medien und Journalismus 1. Eine Einführung, Opladen 1994, S. 295-312.

Berg, Klaus / Marie-Luise Kiefer (Hg.): Massenkommunikation IV. Eine Langzeitstudie zur Mediennutzung und Medienbewertung 1964-1990, Baden-Baden 1992.

Berger, C. R. / S. H. Chaffee (Hg.): Handbook of Communication Science, Newbury Park, CA. 
1987.

Berkowitz, Leonard (Hrsg.): Advances in Experimental Social Psychology, Vol. 14, New York/London 1981.

Berkowitz, Leonard (Hrsg.): Advances in Experimental Social Psychology, Vol. 19, New York/London 1986.

Berlo, David K. : The Process of Communication, New York 1960.

Berlo, David K. / James B. Lemert / Robert J. Mertz: Dimensions for Evaluating the Acceptability of Message Sources, in: Public Opinion Quarterly 33/1969, S. 563-576.

Bernberg, Raymond E.: Prestige Suggestion in Art as Communication, in: Journal of Social Psychology 38/1953, S. 23-30.

Berry, Fred C.: A Study of Accuracy in Local News Stories of Three Dailies, in: Journalism Quarterly 44/1967, S. 482-490.

Berryman-Fink, Cynthia L. / James R. Wilcox: A Multivariate Investigation of Perceptual Attributions Concerning Gender Appropriateness in Language, in: Sex Roles 9/1983, S. 663-681.

Berscheid, Ellen: Opinion Change and Communicator-Communicatee Similarity and Dissimilarity, in: Journal of Personality and Social Psychology 4/1966, S. 670-680.

Berscheid, Ellen / Elaine Walster: Physical Attractiveness, in: Leonard Berkowitz (Hrsg.): Advances in Experimental Social Psychology, Vol. 7, New York / London 1974, S. 157-215.

Birnbaum, Michael H. / Steven E. Stegner: Source Credibility in Social Judgment: Bias, Expertise, and the Judge's Point of View, in: Journal of Personality and Social Psychology 37/1979, S. 48-74.

Birnbaum, Michael H. / Rebecca Wong / Leighton K. Wong: Combining Information from Sources that Vary in Credibility, in: Memory \& Cognition 4/1976, S. 330-336.

Bishop, Robert L. / Mary Boersma / John Williams:Teen-agers and News Media: Credibility Canyon, in: Journalism Quarterly 46/1969, S. 597-599.

Bishop, Robert L. / Sue A. Schultz: The Credibility Gap: Is It Widening? in: Journalism Quarterly 44/1967, S. 740-741.

Bissinger, Manfred: Wenn Bilder lügen, in: Die Woche vom 16.02.1996.

Bitala, Michael: Als die Bilder lügen lernten, in: Süddeutsche Zeitung vom 10./11.02.1996.

Bleda, Paul R.: Toward a Clarification of the Role of Cognitive and Affective Processes in the Similarity-Attraction Relationship, in: Journal of Personality and Social Psychology 29/1974, S. 368-373.

Block, Helen Lewis: Studies in the principles of Judgments and Attitudes: IV. The Operation of "Prestige Suggestion", in: Journal of Social Psychology 14/1941, S. 229-256. 
Bloom, Arvid J. / Jacob E. Hautalouma: Effects of Message Valence, Communicator Credibility, and Source Anonymity on Reactions to Peer Feedback, in: Journal of Social Psychology 127/1987, S. 329-338.

Bochner, Arthur P.: On the Paradigm That Would Not Die, in: James A. Anderson (Hrsg.): Communication Yearbook 14, Newbury Park u.a. 1991, S. 484-491.

Bochner, Stephen / Chester A. Insko: Communicator Discrepancy, Source Credibility, and Opinion Change, in: Journal of Personality and Social Psychology 4/1966, S. 614-621.

Bock, Douglas G. / Thomas J. Saine: The Impact of Source Credibility, Attitude Valence, and Task Sensitization on Trait Errors in Speech Evaluation, in: Speech Monographs 42/1975, S. 229-236.

Böhret, Carl / Werner Jann: Verwaltungsskandale, in: Aus Politik und Zeitgeschichte (Beilage zu 'Das Parlament') B 27/1982, S. 35-52.

Bolesch, Cornelia: Versicherungsfritze Wickert? Nicht dumm, ehrlich! in: Süddeutsche Zeitung vom 14.11.1995.

Borkenau, Peter: Reicher Mann und schöne Frau? Zwei Studien zu Geschlechtsunterschieden in der Partnerpräferenz, in: Zeitschrift für Sozialpsychologie 24/1993, S. 289-297.

Bortz, Jürgen / Konrad Leitner: Zur Frage der Beziehung zwischen der attitüdenändernden Wirkung zweier Tageszeitungen und ihrer Bewertung, in: Zeitschrift für Sozialpsychologie 10/1979, S. 70-84.

Bostian, Lloyd R. / John E. Ross: Functions and Meanings of Mass Media for Wisconsin Farm Women, in: Journalism Quarterly 42/1965, S. 69-76.

Bostrom, Robert N. / John R. Baseheart / Charles M. Rossiter, Jr.: The Effects of Three Types of Profane Language in Persuasive Messages, in: Journal of Communication 23/1973, S. 461475.

Bostrom, Robert N. / Raymond K. Tucker: Evidence, Personality, and Attitude Change, in: Speech Monographs 36/1969, S. 22-27.

Bowers, John Waite / Michael M. Osborn: Attitudinal Effects of Selected Types of Concluding Metaphors in Persuasive Speeches, in: Speech Monographs 33/1966, S. 147-155.

Bowers, John Waite / William A. Phillips: A Note on the Generality of Source-Credibility Scales, in: Speech Monographs 34/1967, S. 185-186.

Bradac, James J. / Catherine W. Konsky / Robert A. Davies: Two Studies of the Effects of Linguistic Diversity upon Judgments of Communicator Attributes and Message Effectiveness, in: Communication Monographs 43/1976, S. 70-79.

Bradley, P. H.: The Folk-Linguistics of Women's Speech: An Empirical Examination, in: Communication Monographs 48/1981, S. 73-90

Brandstätter, Veronika et al.: Indirect Majority and Minority Influence: An Exploratory Study, in: European Journal of Social Psychology 21/1991, S. 199-211. 
Brandt, D. R. / Gerald R. Miller / John E. Hocking: The Truth-Deception Attribution: Effects of Familiarity on the Ability of Observers to Detect Deception, in: Human Communication Research 6/1980, S. 99-110.

Braskamp, Larry A. / Robert D. Brown / Dianna L. Newman: The Credibility of a Local Educational Program Report: Author Source and Client Audience Characteristics, in: American Educational Research Journal 15/1978, S. 441-450.

Bredow, Wilfried von: Legitimation durch Empörung. Vorüberlegung zu einer politischen theorie des Skandals, in: Schoeps (1992), S. 190-208.

Brehm, Jack W. / David Lipsher: Communicator-Communicatee Discrepancy and perceived Communicator Trustworthiness, in: Journal of Personality 27/1959, S. 352-361.

Brock, Timothy / L. A. Becker: Ineffectiveness of "Overheard" Counterpropaganda, in: Journal of Personality and Social Psychology 2/1965, S. 654-660.

Brock, Timothy C.: Communicator-Recipient Similarity and Decision Change, in: Journal of Personality and Social Psychology 1/1965, S. 650-654.

Brosius, Hans-Bernd: Alltagsrationalität in der Nachrichtenrezeption. Ein Modell zur Wahrnehmung und Verarbeitung von Nachrichteninhalten, Opladen 1995.

Broudy, Harry S.: Truth and Credibility. The Citizen's Dilemma, New York/London 1981.

Brown, Bruce L. / William J. Strong / Alvin C. Rencher: Perceptions of Personality from Speech: Effects of Manipulations of Acoustical Parameters, in: Journal of the Acoustical Society of America 54/1973, S. 29-35.

Brown, Bruce L. / William J. Strong / Alvin C. Rencher: Fifty-four Voices from Two: The Effects of Simultaneous Manipulations of Rate, Mean Fundamental Frequency and Variance of Fundamental Frequency on Ratings of Personality from Speech, in: Journal of the Acoustical Society of America 55/1974, S. 313-318.

Brownlow, Sheila / Leslie A. Zebrowitz: Facial Appearance, Gender, and Credibility in Television Commercials, in: Journal of Nonverbal Behavior 14/1990, S. 51-60.

Bryant, Jennings et al.: Relationship Between College Teachers' Use of Humor in the Classroom and Students' Evaluation of Their Teachers, in: Journal of Educational Psychology 72/1980, S. 511-519.

Buckley, John E.: Credibility and Drug Education: A Critique and Reformulation, in: International Journal of the Addictions 24/1989, S. 489-497.

Buller, David B. / Judee K. Burgoon: The Effects of Vocalics and Nonverbal Sensitivity on Compliance. A Replication and Extension, in: Human Communication Research 13/1986, S. 126-144.

Bulitta, Erich / Hildegard Bulitta: Wörterbuch der Synonyme und Antonyme, Frankfurt a.M. 1990.

Bungardt, Thomas H.: Operationalisierung eines Dispositionsbegriffs am Beispiel der Glaubwürdigkeit, Frankfurt a.M. 1981. 
Burgoon, Judee K.: The Ideal Source: A Re-examination of Source Credibility Measurement, in: Central States Speech Journal 27/1976, S. 200-206.

Burgoon, Judee K.: Attributes of the Newscaster's Voice as Predictors of His Credibility, in: Journalism Quarterly 55/1978, S. 276-281, 300.

Burgoon, Judee K.: Nonverbal Communication Research in the 1970s: An Overview, in: Dan Nimmo (Hrsg.): Communication Yearbook 4, New Brunswick / London 1980, S. 179-197.

Burgoon, Judee K. / Thomas Birk / Michael Pfau: Nonverbal Behaviors, Persuasion, and Credibility, in: Human Communication Research 17/1990, S. 140-169.

Burgoon, Michael / Judee K. Burgoon / Miriam Wilkinson: Newspaper Image and Evaluation, In: Journalism Quarterly 58/1981, S. 411-419, 433.

Burgoon, Michael / Lynn Fraedrich / Susan Bachman: Credibility of Communicators and Distraction as Predictors of Change in Attitude, in: Psychological Reports 45/1979, S. 479-482.

Burns, J. A. / B. L. Kintz: Eye Contact While Lying During an Interview, in: Bulletin of the Psychonomic Society 2/1976, S. 87-89.

Burtt, Harold / Don Falkenberg, Jr.: The Influence of Majority and Expert Opinion on Religious Attitudes, in: Journal of Social Psychology 14/1941, S. 269-278.

Byrne, Donn: Interpersonal Attraction and Attitude Similarity, in: Journal of Abnormal and Social Psychology 62/1961, S. 713-715.

Byrne, Donn / Don Nelson: Attraction as a Function of Attitude Similarity-Dissimilarity: The Effect of Topic Importance, in: Psychonomic Science 1/1964, S. 93-94.

Calonego, Bernadette: "So wird's gemacht, Frau Caniga!" Wie ein Schweizer Boulevardblatt gegen eine neue Fernseh-Chefredakteurin agiert, in: Süddeutsche Zeitung vom 19.10.1995.

Cameron, Glen T. / John Eric Haley: Feature Advertising: Policies and Attitudes in Print Media, in: Journal of Advertising 21/1992, S. 47-55.

Capon, Noel / James Hulbert: The Sleeper Effect - An Awakening, in: Public Opinion Quarterly 37/1973, S. 333-358.

Carbone, Tamara: Stylistic Variables as Related to Source Credibility: A Content Analysis Approach, in: Speech Monographs 42/1975, S. 99-106.

Carlson, Earl R.: Word Familiarity as a Factor in Forming Impressions, in: Psychological Reports 7/1960, S. 18.

Carr, John E.: Differentiation as a Function of Source Characteristics and Judge's Conceptual Structure, in: Journal of Personality 37/1969, S. 378-386.

Carter, Richard F. / Bradley S. Greenberg: Newspaper or TV: Which Do You Believe? in: Journalism Quarterly 42/1965, S. 29-34.

Catano, Victor M.: Effectiveness of Verbal Praise as a Function of Expertise of its Source, in: Perceptual and Motor Skills 42/1976, S. 1283-1286.

Cathcart, Robert S.: An Experimental Study of the Relative Effectiveness of Four Methods of 
Presenting Evidence, in: Speech Monographs 22/1955, S. 227-233.

Chaiken, Shelly: Communicator Physical Attractiveness and Persuasion, in: Journal of Personality and Social Psychology 37/1979, S. 1387-1397.

Chaiken, Shelly: Heuristic Versus Systematic Information Processing and the Use of Source Versus Message Cues in Persuasion, in: Journal of Personality and Social Psychology 39/1980, S. 752-766.

Chaiken, Shelly: The Heuristic Model of Persuasion, in: Mark P. Zanna / James M. Olson / C. Peter Herman (Hg.): Social Influence: The Ontario Symposium, Vol. 5, Hillsdale, NJ. 1987, S. 339.

Chaiken, Shelly / Alice H. Eagly: Communication Modalty as a Determinant of Message Persuasiveness and Message Comprehensibility, in: Journal of Personality and Social Psychology 34/1976, S. 605-614.

Chaiken, Shelly / Alice H. Eagly: Communication Modalty as a Determinant of Persuasion: The Role of Communicator Salience, in: Journal of Personality and Social Psychology 45/1983, S. 241-256.

Chang, Lawrence K. H. / James B. Lemert: The Invisible Newsman and other Factors in Media Competition, in: Journalism Quarterly 45/1968, S. 436-444.

Charnley, Mitchell V.: Preliminary Notes on A Study of Newspaper Accuracy, in: Public Opinion Quarterly 13/1936, S. 394-401.

Chebat, Jean Charles / Pierre Filiatraut / Jean Perrien: Limits of Credibility: The Case of Political Persuasion, in: Journal of Social Psychology 130/1990, S. 157-167.

Choo, Tong-He: Communicator Credibility and Communication Discrepancy as Determinants of Opinion Change, in: Journal of Social Psychology 64/1964, S. 65-76.

Clark, Russell D., III / Anne Maass: The Role of Social Categorization and Perceived Source Credibility in Minority Influence, in: European Journal of Social Psychology 18/1988, S. 381-394.

Cody, Michael J. / H. D. O'Hair: Nonverbal Communication and Deception: Differences in Deception Cues Due to Gender and Communicator Dominance, in: Communication Monographs 50/1983, S. 175-192.

Cody, Michael J. / Peter J. Marston / Myrna Foster: Deception: Paralinguistic and Verbal Leakage, in: Robert N. Bostrom / Bruce H. Westley (Hg.): Communication Yearbook 8, Beverly Hills / London / New Delhi 1984, 464-490.

Cole, David: 'Rational Argument' and 'Prestige-Suggestion' as Factors Influencing Judgment, in: Sociometry 17/1954, S. 350-354.

Collodi, Carlo: Das Märchen vom Pinoccio, Zürich 1968.

Comadena Mark E.: Accuracy in Detecting Deception: Intimate and Friendship Relations, in: Michael Burgoon / Noel Doran (Hg.): Communication Yearbook 6, Beverly Hills / London / New Delhi 1982, S. 446-472. 
Constanzo, Mark et al.: Energy Conservation Behavior. The Difficult Path From Information to Action, in: American Psychologist 41/1986, S. 521-528.

Cook, Thomas D.: Competence, Counterarguing, and Attitude Change, in: Journal of Personality 37/1969, S. 342-358.

Cook, Thomas D. et al.: History of the Sleeper Effect: Some Logical Pitfalls in Accepting the Null Hypothesis, in: Psychological Bulletin 86/1979, S. 662-679.

Covello, Vincent T. / David B. McCallum / Maria T. Pavlova (Hg.): Effective Risk Communication. The Role and Responsibility of Government and Nongovernment Organizations, New York/London 1989.

Crano, William D.: Effects of Sex, Response Order, and Expertise in Conformity: A Dispositional Approach, in: Sociometry 33/1970, S. 239-252.

Crisci, Richard / Howard Kassinove: Effects of Perceived Expertise, Strength of Advice, and Environmental Setting on Parental Compliance, in: Journal of Social Psychology 89/1973, S. 245-250.

Cronkhite, Gary / Jo Liska: A Critique of Factor Analytic Approaches to the Study of Credibility, in: Communication Monographs 43/1976, S. 91-107.

Cronkhite, Gary / Jo Liska: The Judgment of Communicant Acceptability, in: M. E. Roloff / G. R. Miller (Hg.): Persuasion: New Directions in Theory and Research, Newbury Park, CA. 1980, S. 100-139.

Cutrow, R. J. et. al.: The Objective Use of Multiple Physiological Indices in the Detection of Deception, in: Psychophysiology 9/1972, S. 578-588.

Dabbs, James M., Jr.: Self-Esteem, Communicator Characteristics, and Attitude Change, in: Journal of Abnormal and Social Psychology 69/1964, S. 173-181.

Dancer, F. E. X. (Hrsg.): Human Communication Theory, New York 1982.

Dean, Robert / John Austin / William Watts: Forewarning Effects in Persuasion: Field and Classroom Experiments, in: Journal of Personality and Social Psychology 18/1971, S. 210221.

Deimling, Susanne / Jürgen Bortz / Gerhard Gmel: Zur Glaubwürdigkeit von Fernsehanstalten. Entwicklung und Erprobung eines Erhebungsinstruments, in: Medienpsychologie 5/1993, S, 203-219.

Delia, Jesse G.: A Constructivist Analysis of the Concept of Credibility, in: Quarterly Journal of Speech 62/1976, S. 361-375.

Delia, Jesse G. et al.: The Dependency of Interpersonal Evaluations on Context Relevant Beliefs about the Other, in: Communication Monographs 42/1975, S. 10-19. 
Delia, Jesse G. / Barbara J. O'Keefe / Daniel J. O'Keefe: The Constructivist Approach to Communication, in: F. E. X. Dancer (Hrsg.): Human Communication Theory, New York 1982, S. 147-191.

Dembo, Richard et al.: Drug Information Source Credibility among Junior and Senior High School Youths, in: American Journal of Drug and Alcohol Abuse 4/1977, S. 43-54. [1977a]

Dembo, Richard et al.: The Believability of the Media as Sources of Information on Drugs, in: The International Journal of the Addictions 12/1977, S. 959-969. [1977b]

DePaulo, Bella M. / Robert Rosenthal: Telling Lies, in: Journal of Personality and Social Psychology 37/1979, S. 1713-1722.

DePaulo, Bella M. / Miron Zuckerman / Robert Rosenthal: Humans as Lie Detectors, in: Journal of Communication 30/1980, S. 129-139.

DePaulo, Bella M. / Miron Zuckerman / Robert Rosenthal: The Deceptions of Everyday Life, in: Journal of Communication 30/1980, S. 216-218.

DePaulo, Peter J.: Research on Deception in Marketing Communications: Its Relevance to the Study of Nonverbal Behavior, in: Journal of Nonverbal Behavior 12/1988, S. 253-273.

Dholakia, Ruby Roy / Brian Sternthal: High Credible Souces: Persuasive Faciliators or Persuasive Liabilities? in: Journal of Consumer Research 3/1977, S. 223-232.

Dion, Karen K. / Ellen Berscheid / Elaine Walster: What is Beautiful is Good, in: Journal of Personality and Social Psychology 24/1972, S. 285-290.

Dion, Kenneth L. / Karen Dion: Belief in a Just World and Physical Attractiveness Stereotyping, in: Journal of Personality and Social Psychology 52/1987, S. 775-780.

Di Vesta, Francis J. / Donald L. Meyer / Judson MillsConfidence in an Expert as a Function of his Judgements, in: Human Relations 17/1964, S. 235-242.

Donsbach, Wolfgang et al.: Beziehungsspiele - Medien und Politik in der öffentlichen Diskussion. Fallstudien und Analysen, Gütersloh 1993.

Drescher, Siegfried H.: Wirkung des Fernsehens als Quelle aktueller Informationen, in: Publizistik 14/1969, S. 173-184.

Drinkmann, Arno / Norbert Groeben: Metaanalysen für Textwirkungsforschung. Methodologische Varianten und inhaltliche Ergebnisse im Bereich der Persuasionswirkung von Texten, Weinheim 1989.

Durand, Richard M. / Jesse E. Teel, Jr. / William O. Bearden: Racial Differences in Perceptions of Media Advertising Credibility, in: Journalism Quarterly 56/1979, S. 562-566.

Dutton, Donald G.: The Maverick Effect: Increased Communicator Credibility as a Result of Abandoning a Career, in: Canadian Journal of Behavioral Science 5/1973, S. 145-151.

Eagly, Alice H.: Comprehensibility of Persuasive Arguments as a Determinant of Opinion Change, in: Journal of Personality and Social Psychology 29/1974, S. 758-773. 
Eagly, Alice H. / Shelly Chaiken: An Attribution analysis of the Effect of Communicator Characteristics on Opinion Change: The Case of Communicator Attractiveness, in: Journal of Personality and Social Psychology 32/1975, S. 136-144.

Eagly, Alice H. / Shelly Chaiken: Why Would Anyone Say that? Causal Attribution of Statements about the Watergate Scandal, in: Sociometry 39/1976, S. 236-243.

Eagly, Alice H. / Wendy Wood / Shelly Chaiken: Causal Inferences About Communicators and Their Effect on Opinion Change, in: Journal of Personality and Social Psychology 36/1978, S. 424-435.

Ebbinghausen, Rolf / Sighard Neckel (Hg.): Anatomie des politischen Skandals, Frankfurt a.M. 1989.

Edelstein, Alex S.: An Alternative Approach to the Study of Source Effects in MASS Communication, in: Studies of Broadcasting 9/1973, S. 5-29.

Edelstein, Alex S.: The Uses of Communication in Decision-Making. A Comparative Study of Yugoslavia and the United States, New York 1974.

Edelstein, Alex S.: An Alternative Approach to the Study of Source Effects in MASS Communication, in: Communications 4/1978, S. 71-90.

Edelstein, Alex S. / Dianne P. Tefft: Media Credibility and Respondent Credulity with Respect to Watergate, in: Communication Research 1/1974, S. 426-439.

Edwards, James: Print Media Use and Perceived Credibility among Senior Congressional Stuff. Unpublished Doctoral Dissertation, University of Tennesse 1992.

Ehmig, Simone Christiane: Zitateinbindung im SPIEGEL, Mainz 1989 (unveröffentlichte Magisterarbeit).

Eisinger, Richard / Judson Mills: Perception of the Sincerity and Competence of a Communicator as a Function of the Extremity of His Position, in: Journal of Experimental Social Psychology 4/1968, S. 224-232.

Ekman, Paul: Weshalb Lügen kurze Beine haben. Über Täuschungen und deren Aufdeckung im privaten und öffentlichen Leben, Berlin/New York 1989. [Im Original: Telling Lies. Clues to Deceit in the Marketplace, Politics, and Mariage, New York 1985.]

Ekman, Paul / Wallace V. Friesen: Nonverbal Leakage and Clues to Deception, in: Psychatry 32/1969, S. 88-105.

Ekman, Paul / Wallace V. Friesen: Hand Movements, in: Journal of Communication 22/1972, S. 353-374.

Ekman, Paul / Wallace V. Friesen: Detecting Deception from the Body or Face, in: Journal of Personality and Social Psychology 29/1974, S. 288-298.

Ekman, Paul / Wallace V. Friesen / Klaus Scherer: Body Movement and Voice Pitch in Deceptive Interaction, in: Semiotica 16/1976, S. 23-27.

Ekman, Paul et al.: Relative Importance of Face, Body and Speech in Judgments of Personality and Affect, in: Journal of Personality and Social Psychology 38/1980, S. 270-277. 
Engstrom, Erika: Effects of Sex and Appearance of Ratings of Source Credibility. Unpublished Doctoral Dissertation, University of Florida 1991.

Erskine, Hazel: The Polls: Opinion of the News Media, in: Public Opinion Quarterly 34/1970, S. 630-643.

Fabris, Hans Heinz: Massenmedien - Instrumente der "Skandalisierung" oder "Vierte Gewalt"? Zum Kontrollpotential der Medien, in: Christian Brünner (Hrsg.): Korruption und Kontrolle, Wien 1981, S. 239-264.

Feather, N.T.: Positive and Negative Reactions to Male and Female Success and Failure in Relation to the Perceived Status and Sex-Typed Appropriateness of Occupations, in: Journal of Personality and Social Psychology 31/1975, S. 536-548.

Feldman, Robert S. / Linda Devin-Sheehan / Vernon L. Allen: Nonverbal Cues as Indicators of Verbal Dissembling, in: American Educational Research Journal 15/1978, S. 217-231.

Feldman, Robert S. / S. L. Jenkins / O. Popoola: Detection of Deception in Adults and Children via Facial Expressions, in: Child Development 49/1979, S. 1107-1113.

Feldman, Robert S. / John B. White: Detecting Deception in Children, in: Journal of Communication 30/1980, S. 121-128.

Feldman-Summers, Shirley et al.: Influence Attempts Whwn Competing Views Are Gender-Related: Sex as Credibility, in: Psychology of Women Quarterly 5/1980, S. 311-320.

Fine, Bernard J.: Conclusion-Drawing, Communicator Credibility, and Anxiety as Factors in Opinion Change, in: Journal of Abnormal and Social Psychology 54/1957, S. 369-374.

Friedman, Hershey H. / I. Friedman: Whom Do Students Trust? in: Journal of Communication 26/1976, S. 48-49

Friedman, Hershey H. / Michael J. Santeramo / Anthony Traina: Correlates of Trustworthiness for Celebrities, in: Journal of Academy of Marketing Science 6/1979, S. 291-299.

Friedman, Howard S. / Timothy I. Mertz / M. Robin DiMatteo: Perceived Bias in the Facial Expressions of Television News Broadcasters, in: Journal of Communication 30/1980, S. 103-111.

Fulton, R. Barry: The Measurement of Speaker Credibility, in: Journal of Communication 20/1970, S. 270-279.

Furnham, Adrian / Nadine Bitar: The Stereotyped Portrayal of Men and Women in British Television Advertisements, in: Sex Roles 29/1993, S. 297-310.

Furnham, Adrian / Sandra Schofield: Sex-Role Stereotyping in British Radio Advertisements, in: British Journal of Social Psychology 25/1986, S. 165-171.

Furnham, Adrian / Virginia Voli: Gender Stereotypes in Italian Television Advertisements, in: Journal of Broadcasting \& Electronic Media 33/1989, S. 175-185.

QGantz, Walter: The Influence of Researcher Methods on Television and Newspaper News Credibility Evaluations, in: Journal of Broadcasting 25/1981, S. 155-169. 
Gaziano, Cecilie: How Credible Is the Credibility Crisis? in: Journalism Quarterly 65/1988, S. 267278,375 .

Gaziano, Cecilie / Kristin McGrath: Measuring the Concept of Credibility, in: Journalism Quarterly 63/1986, S. 451-462.

Gaziano, Cecilie / Kristin McGrath: Newspaper Credibility and Relationships Of Newspaper Journalists to Communities, in: Journalism Quarterly 64/1987, S. 317-328, 345.

Gelb, Betsy D. / Charles M. Pickett: Attitude-Toward-The-Ad: Links to Humor and to Advertising Effectiveness, in: Journal of Advertising 12/1983, S. 34-42.

Gelb, Betsy D. / George M. Zinkhan: Humor and Advertising Effectiveness after Repeated Exposures to a Radio Commercial, in: Journal of Advertising 15/1986, S. 15-20, 34.

Gerhards, Maria / Walter Klingler: Politikmagazine im öffentlich-rechlichen Fernsehen, in: Media Perspektiven 4/1995, S. 166-171.

Giffin, Kim: The Contribution of Studies of Source Credibility to a Theory of Interpersonal Trust in the Communication Process, in: Psychological Bulletin 68/1967, S. 104-120.

Gilkinson, Howard / Stanley F. Paulson / Donald E. Sikkink: Effects of Order and Authority in an Argumentative Speech, in: Quarterly Journal of Speech 40/1954, S. 183-192.

Gillen, Barry: Physical Attractiveness: A Determinant of Two Types of Goodness, in: Personality and Social Psychology Bulletin 7/1981, S. 277-281.

Gillig, Paulette M. / Anthony G. Greenwald: Is it Time to Lay the Sleeper Effect to Rest? in: Journal of Personality and Social Psychology 29/1974, S. 132-139.

Gössmann, Wilhelm: Glaubwürdigkeit im Sprachgebrauch. Stilkritische und sprachdidaktische Untersuchungen, München 1970.

Götsch, Katja: Riskantes Vertrauen. Theoretische und empirische Untersuchung zum Konstrukt Glaubwürdigkeit, Münster/Hamburg 1994.

Goldberg, Herbert / Marvin A. Iverson: Inconsitency in Attitude of High Status Persons and Loss of Influence: An Experimental Study, in: Psychological Reports 16/1965, S. 673-683.

Gorn, Gerald J.: The Effects of Personal Involvement, Communication Discrepancy, and Source Prestige on Reactions to Communications on Separatism, in: Canadian Journal of Behavioral Science 7/1975, S. 369-386.

Gotlieb, Jerry B. / Alan J. Dubinsky: Influence of Price on Aspects of Consumers' Cognitive Process, in: Journal of Applied Psychology 76/1991, S. 541-549.

Gotlieb, Jerry B. / Dan Sarel: Comparative Advertising Effectiveness: The Role of Involvement and Source Credibility, in: Journal of Advertising 20/1991, S. 38-45.

Gotlieb, Jerry B. / Dan Sarel: The Influence of Type of Advertisement, Price, and Source Credibility on Perceived Quality, in: Journal of the Academy of Marketing Science 20/1992, S. 253260.

Greenberg, Bradley S.: Media Use and Believability: Some Multiple Correlates, in: Journalism Quarterly 43/1966, S. 665-670. 
Greenberg, Bradley S. / Gerald R. Miller: The Effects of Low-Credible Sources on Message Acceptance, in Speech Monographs 33/1966, S. 127-136.

Greenberg, Bradley S. / Edward L. Razinsky: Some Effects of Variations in Message Quality, in: Journalism Quarterly 43/1966, S. 486-492.

Greenberg, Bradley S. / Percy H. Tannenbaum: The Effects of Bylines on Attitude Change, in: Journalism Quarterly 38/1961, S. 535-537.

Greenberg, J. / A. Wursten: The Psychologist and the Psychiatrist as Expert Witnesses: Perceived Credibility and Influence, in: Professional Psychology: Research and Practice 19/1988, S. 373-378.

Groth, Otto: Die unerkannte Kulturmacht. Grundlegung der Zeitungswissenschaft (Periodik), Band 1: Das Wesen des Werkes, Berlin 1960.

Grotta, Gerald L.: Attitudes on Newspaper Accuracy and External Controls, in: Journalism Quarterly 46/1969, S. 757-759.

Gruder, Charles L. et al.: Empirical Tests of the Absolute Sleeper Effect Predicted from the Discounting Cue Hypothesis, in: Journal of Personality and Social Psychology 36/1978, S. 1061-1074.

Gruner, Charles R.: Effect of Humor on Speaker Ethos and Audience Information, in: Journal of Communication 17/1967, S. 228-233.

Gunther, Albert: Attitude Extremity and Trust in Media, in: Journalism Quarterly 65/1988, S. 279287.

Gunther, Albert / Dominic L. Lasorsa: Issue Importance and Trust in Mass Media, in: Journalism Quarterly 63/1986, S. 844-848.

Gunther, Albert C.: Biased Press or Biased Public? Attitudes toward Media Coverage of Social Groups, in: Public Opinion Quarterly 56/1992, S. 147-167.

Hagen, Lutz M.: Die opportunen Zeugen. Konstruktionsmechanismen von Bias in der Zeitungsberichterstattung über die Volkszählungsdiskussion, in: Publizistik 37/1992, S. 444-460.

Haiman, Franklyn S.: An Experimental Study of the Effects of Ethos in Public Speaking, in: Speech Monographs 16/1949, S. 190-202.

Haimerl, Norbert: Schreiben sie wirklich alle dasselbe? Auf der Suche nach Konsonanz und Publizistischem Spektrum. Vergleichende Inhaltsanalyse der Berichterstattung von vier deutschen Tageszeitungen anläßlich des Reykjavik-Treffens von Ronald Reagan und Michail Gorbatschow im Oktober 1986, München 1988 (unveröffentlichte Magisterarbeit).

Hall, J. A.: Gender Effects in Decoding Nonverbal Cues, in: Psychological Bulletin 3/1977, S. 446449. 
Haller, Michael: Wie wissenschaftlich ist Wissenschaftsjournalismus? Zum Problem wissenschaftsbezogener Arbeitsmethoden im tagesaktuellen Journalismus, in: Publizistik 32/1987, S. 305-319.

Hamilton, Mark A. / John E. Hunter / Michael Burgoon: An Empirical Test of an Axiomatic Model of the Relationship Between Language Intensity and Persuasion, in: Journal of Language and Social Psychology 9/1990, S. 235-255.

Hammond, Sharon Lee: Health Advertising: The Credibility of Organizational Sources, in: Margaret L. McLaughlin (Hrsg.): Communication Yearbook 10, Newbury Park u.a. 1987, S. 613628.

Hance, B. J. / C. Chess / P. M. Sandman: Improving Dialogue with Communities: A Risk Communication Manual for Government, in: Vincent T. Covello / David B. McCallum / Maria T. Pavlova (Hg.): Effective Risk Communication. The Role and Responsibility of Government and Nongovernment Organizations, New York/London 1989, S. 195-285.

Hankiss, Agnes: Games Con Men Play: The Semiosis of Deceptive Interaction, in: Journal of Communication 30/1980, S. 104-112.

Hanneman, Gerhard J.: Communicating Drug-Abuse Information among College Students, in: Public Opinion Quarterly 37/1973, S. 171-191.

Hansen, Robert A. / Carol A. Scott: Comments on "Attribution Theory and Advertiser Credibility", in: Journal of Marketing Research 13/1976, S. 193-197.

Harmon, Robert R. / Kenneth A. Coney: The Persuasive Effects of Source Credibility in Buy and Lease Situations, in: Journal of Marketing Research 19/1982, S. 255-260.

Harris, Mary B. et al.: Clothing: Communication, Compliance, and Choice, in: Journal of Applied Social Psychology 13/1983, S. 88-97.

Harvey, J. / D. Hays: Effect on Dogmatism and Authority of the Source of Communication upon Persuasion, in: Psychological Reports 30/1972, S. 119-122.

Hass, R. G. / K. Grady: Temporal Delay, Type of Forewarning and Resistance to Influence, in: Journal of Experimental Social Psychology 11/1975, S. 459-469.

Hassebrauck, Manfred: Die Beurteilung der physischen Attraktivität: Konsens unter Urteilern? In: Zeitschrift für Sozialpsychologie 14/1983, S. 152-161.

Hassebrauck, Manfred: Der Einfluß von Attitüdenähnlichkeit und physischer Attraktivität auf die Beurteilung von Personen, in: Psychologische Beiträge 27/1986, S. 265-276.

Hassebrauck, Manfred: Der Einfluß von Attitüdenähnlichkeit auf die Beurteilung physischer Attraktivität, in: Zeitschrift für Differentielle und Diagnostische Psychologie 7/1986, S. 8998. [1986a]

Hassebrauck, Manfred: Perception of Physical Attractiveness Influenced by Similarity of Attitudes, in: Perceptual and Motor Skills 63/1986, S. 185-186. [1986b]

Hassebrauck, Manfred: Die Beurteilung der physischen Attraktivität, in: Hassebrauck / Niketta (1993), S. 29-59. 
Hassebrauck, Manfred / Reiner Niketta (Hg.): Physische Attraktivität, Göttingen u.a. 1993.

Hayano, David M.: Communicative Competency Among Poker Players, in: Journal of Communication 30/1980, S. 113-120.

Heath, Robert L. / Jennings Bryant: Human Communication Theory and Research. Concepts, Contexts, and Challenges, Hillsdale, N.J. 1992.

Heesacker, Martin / Richard E. Petty / John T. Cacioppo: Field Dependence and Attitude Change: Source Credibility Can Alter Persuasion by Affecting Message-Relevant Thinking, in: Journal of Personality 51/1983, S. 653-666.

Hegner, Cathrin: 'Deutsche Ansichten'. Stereotype und die Darstellung von Ostdeutschen in 'Stern' und 'Bunte'. Eine vergleichende Inhaltsanalyse, München 1994 (unveröffentlichte Magisterarbeit).

Hendrick, Clyde / David R. Shaffer: Effects of Arousal and Credibility on Learning and Persuasion, in: Psychonomic Science, 20/1970, S. 241-243.

Hennig, John: Sprache und Glaubwürdigkeit, in: Zeitschrift für deutsche Sprache 22/1966, S. 112121.

Hennigan, Karen M. / Thomas D. Cook / Charles L. Gruder: Cognitive Tuning Set, Source Credibility, and the Temporal Persistence of Attitude Change, in: Journal of Personality and Social Psychology 42/1982, S. 412-425.

Hensley, Wayne E.: A Criticism of 'Dimensions of Source Credibility: A Test for Reproducibility', in: Speech Monographs 41/1974, S. 293-294.

Heringer, Hans Jürgen: "Ich gebe Ihnen mein Ehrenwort". Politik - Sprache - Moral, München 1990.

Hesse, Kurt Rolf: Nutzung und Image des "Westfernsehens" bei DDR-Übersiedlern, in: Media Perspektiven 4/1986, S. 265-272.

Hewgill, Murray A. / Gerald R. Miller: Source Credibility and Response to Fear-Arousing Communications, in: Speech Monographs 32/1965, S. 95-101.

Higgins, E. Tory / C. Peter Herman / Mark P. Zanna (Hg.): Social Cognition. The Ontario Symposium, Vol 1, Hillsdale N.J. 1981.

Hill, Arthur H.: Credibility, Discrepancy and Latitude of Communication as Dimensions of Dissonance Influencing Attitude Change, in: Australian Journal of Psychology 15/1963, S. 124-132.

Hitzler, Ronald: Skandal ist Ansichtssache. Zur Inszenierungslogik ritueller Spektakel in der Politik, in: Ebbinghausen / Neckel (1989), S. 334-354.

Hitzler, Ronald: Skandal: Karrierebremse oder Karrierevehikel? Inszenierungsprobleme Bonner Parlamentarier, in: Sozialwissenschaftliche Informationen 1/1987, S. 22-27.

Hocking, John E. et al.: Detecting Deceptive Communication from Verbal, Visual, and Paralinguistic Cues, in: Human Communication Research 6/1979, S. 33-46. 
Hocking, John E. / D. G. Leathers: Nonverbal Indicators of Deception: A New Theoretical Perspective, in: Communication Monographs 47/1980, S. 119-131.

Hodgson, Shari / Bert Pryor: Sex Discrimination in the Courtroom: Attorney's Gender and Credibility, in: Psychological Reports 55/1984, S. 483-486.

Holahan, Carole Kovalic / Cookie White Stephan: When Beauty isn't Talent: The Influence of Physical Attractiveness, Attitudes Toward Women, and Competence on Impression Formation, in: Sex Roles 7/1981, S. 867-876.

Homer, Pamela M. / Lynn R. Kahle: Source Expertise, Time of Source Identification, and Involvement in Persuasion: An Elaborative Processing Perspective, in: Journal of Advertising 19/1990, S. 30-39.

Hondrich, Karl Otto: Skandalmärkte und Skandalkultur, in: Max Haller / Hans-Joachim HoffmannNowotny / Wolfgang Zapf (Hg.): Kultur und Gesellschaft. Verhandlungen des 24. Deutschen Soziologentags, des 11. Österreichischen Soziologentags und des 8. Kongresses der Schweizerischen Gesellschaft in Zürich 1988, Frankfurt a.M./New York 1989, S. 575-586.

Hondrich, Karl Otto: Skandale als gesellschaftliche Lernmechanismen, in: Schoeps (1992), S. 175189.

Horai, Joann / Nicholas Naccari / Elliot Fatoullah: The Effects of Expertise and Physical Attractiveness upon Opinion Agreement and Liking, in: Sociometry 37/1974, S. 601-606.

Hovland, Carl I. / Irving L. Janis / Harold H. Kelley: Communication and Persuasion. Psychological Studies of Opinion Change, New Haven 1953.

Hovland, Carl I. / Arthur A. Lumsdaine / Fred D. Sheffield: Experiments in Mass Communication, New York 1949.

Hovland, Carl I. / Wallace Mandell: An Experimental Comparison of Conclusion-Drawing by the Communicator and by the Audience, in: Journal of Abnormal and Social Psychology 47/1952, S. 581-588.

Hovland, Carl I. / Henry A. Pritzker: Extent of Opinion Change as a Function of Amount of Change Advocated, in: Journal of Abnormal and Social Psychology 54/1957, S. 257-261.

Hovland, Carl I. / Walter Weiss: The Influence of Source Credibility on Communication Effectiveness, in: Public Opinion Quarterly 15/1951, S. 635-650.

Howard, Carolyn R. / Stanley H. Cohen / Norman Cavior: More Results on Increasing the Persuasiveness of a Low Prestige Communicator: The Effects of the Communicator's Physical Attractiveness and Sex of the Receiver, in: Personality and Social Psychology Bulletin 1/1975, S. 393-395.

Hurwitz, Steven D. / Murray S. Miron / Blair T. Johnson: Source Credibility and the Language of Expert Testimony, in: Journal of Applied Social Psychology 22/1992, S. 1909-1939.

Husek, T. R.: Persuasive Impacts of Early, Late, or No Mention of a Negative Source, in: Journal of Personality and Social Psychology 2/1965, S. 125-128.

Infante, Dominic A.: Persuasion as a Function of the Receiver's Prior Success or Failure as a 
Message Source, in: Communication Quarterly 24/1976, S. 21-26.

Infante, Dominic A.: The Construct Validity of Semantic Differential Scales for the Measurement of Source Credibility, in: Communication Quarterly 28/1980, S. 19-26.

Infante, Dominic A. et al.: A Comparison of Factor and Functional Approaches to Source Credibility, in: Communication Quarterly 31/1983, S. 43-48.

Infante, Dominic A. / Andrew S. Rancer / Deanna F. Womack: Building Communication Theory, Illinois ${ }^{2} 1993$.

Iyengar, Shanto / Donald R. Kinder: Psychological Accounts of Agenda-Setting, in: Sidney Kraus / Richard M. Perloff (Hg.): Mass Media and Political Thought. An Information-Processing Approach, Beverly Hills / London / New Delhi 1985, S. 117-140.

Izard, Ralph S.: Public Confidence in the News Media, in: Journalism Quarterly 62/1985, S. 247255.

Jacobi, Claus: "Fragt, was dem Leser wohltut, was er braucht, um seinen Alltag zu verstehen und zu ertragen", in: 40 Jahre BILD, Sonderdruck vom 26.06.1992.

Jacobs, Alfred et al.: Anonymous Feedback: Credibility and Desirability of Structured Emotional and Behavioral Feedback Delivered in Groups, in: Journal of Counseling Psychology 21/1974, S. 106-111.

Jacobson, Harvey K.: Mass Media Believability: A Study of Receiver Judgements, in: Journalism Quarterly 46/1969, S. 20-28.

Jäckel, Michael / Peter Winterhoff-Spurk (Hg.): Politik und Medien. Analysen zur Entwicklung der politischen Kommunikation, Berlin 1994.

Jellison, Jerald M. / Judson Mills: Effect of Similarity and Fortune of the Other on Attraction, in: Journal of Personality and Social Psychology 5/1967, S. 459-463.

Johannsen, Uwe: Das Marken- und Firmen-Image. Theorie, Methode, Praxis, Berlin 1971.

Johnson, Craig / Larry Vinson: "Damned if You Do, Damned if You Don't?" Status, Powerful Speech, and Evaluations of Female Witnesses, in: Women's Studies in Communication 10/1987, S. 37-44.

Johnson, Homer H. / Richard R. Izzett: Relationship between Authoritarianism and Attitude Change as a Function of Source Credibility and Type of Communication, in: Journal of Personality and Social Psychology 13/1969, S. 317-321.

Johnson, Homer H. / Richard R. Izzett: The Effects of Source Identification on Attitude Change as a Function of the Type of Communication, in Journal of Social Psychology 86/1972, S. 8187.

Johnson, Homer H. / John A. Scileppi: Effects of Ego-Involvement Conditions on Attitude Change to High and Low Credibility Communicators, in: Journal of Personality and Social Psychology 13/1969, S. 31-36.

Johnson, Homer H. / Ivan D. Steiner: Some Effects of Discrepancy Level on Relationships between Authoritarianism and Conformity, in: Journal of Social Psychology 73/1967, S. 199-204. 
Johnson, Homer H. / Ivan D. Steiner: The Effects of Source on Responses to Negative Information About One's Self, in: Journal of Social Psychology 74/1968, S. 215-224.

Johnson, Homer H. / James M. Torcivia / Mary Ann Poprick: Effects of Source Credibility on the Relationship between Authoritarianism and Attitude Change, in: Journal of Personality and Social Psychology 9/1968, S. 179-183.

Johnson, Thomas J.: Exploring Media Credibility: How Mediea and Nonmedia Workers Judged Media Performances in Iran/Contra, in: Journalism Quarterly 70/1993, S. 87-97.

Joseph, W. Benoy: Effect of Communicator Physical Attractiveness and Expertness on Opinion Change and Information Processing. Unpublished Doctoral Dissertation, Columbus: Ohio State University 1977.

Joseph, W. Benoy: The Credibility of Physically Attractive Communicators: A Review, in: Journal of Advertising 11/1982, S. 15-24.

Julian, James W. / Edwin P. Hollander / C. Robert Regula: Endorsement of the Group Spokesman as a Function of His Source of Authority, Competence, and Success, in: Journal of Personality and Social Psychology 11/1969, S. 42-49.

Jungermann, Helmut / Roger E. Kasperson / Peter M. Wiedemann (Hg.): Themes and Tasks of Risk Communication. Proceedings of the International Workshop on Risk Communication held at the KFA Jülich, October 17-21, 1988, Jülich 1988.

Jungermann, Helmut / Bernd Rohrmann / Peter M. Wiedemann (Hg.): Risiko-Konzepte, RisikoKonflikte, Risiko-Kommunikation, Jülich 1990.

Jurma, William E.: Evaluations of Credibility of the Source of a Message, in: Psychological Reports 49/1981, S. 778

Käsler, Dirk et al.: Der politische Skandal. Zur symbolischen und dramaturgischen Qualität von Politik, Opladen 1991.

Kamins, Michael A.: An Investigation into the "Match-Up" Hypothesis in Celebrity Advertising: When Beauty May be Only Skin Deep, in: Journal of Advertising 19/1990, S. 4-13.

Kamins, Michael A. et al.: Two-Sided Versus One-Sided Celebrity Endorsements: The Impact on Advertising Effectiveness and Credibility, in: Journal of Advertising 18/1989, S. 4-10.

Kaplan, Robert M.: Is Beauty Talent? Sex Interaction in the Attractiveness Halo Effect, in: Sex Roles 4/1978, S. 195-204.

Kasperson Roger E./ Peter Jan M. Stallen (Hg.): Communicating Risks to the Public. International Perspectives, Dordrecht 1991.

Katz, Elihu: The Two Step Flow of Communication: An Up-to-date Report on an Hypothesis, in: Public Opinion Quarterly 21/1957, S. 61-78

Kelley, Harold H. / C. L. Woodruff: Members' Reactions to Apparent Group Approval of a Counternorm Communication, in: Journal of Abnormal and Social Psychology 52/1956, S. 67-74.

Kelly, George A.: The Psychology of Personal Constructs, 2 Vols., New York 1955. 
Kelman, Herbert C.: Compliance, Identification, and Internalization: Three Processes of Opinion Change, in: Journal of Conflict Resolution 2/1958, S. 51-60.

Kelman, Herbert C.: Process of Opinion Change, in: Public Opinion Quarterly 25/1961, S. 57-78.

Kelman, Herbert C. / Alice H. Eagly: Attitude toward the Communicator, Perception of Communication Context, and Attitude Change, in: Journal of Personality and Social Psychology 1/1965, S. 63-78.

Kelman, Herbert C. / Carl I. Hovland: Reinstatement of the Communicator in Delayed Measurement of Opinion Change, in: Journal of Abnormal and Social Psychology 48/1953, S. 327-335.

Kennedy, Allan James: An Experimental Study of the Effect of Humorous Message Content Upon Ethos and Persuasiveness, Unpublished Doctoral Dissertation, University of Michigan 1972.

Kepplinger, Hans Mathias: Die aktuelle Berichterstattung des Hörfunks. Eine Inhaltsanalyse der Abendnachrichten und politischen Magazine, Freiburg/München 1985.

Kepplinger, Hans Mathias: Darstellungseffekte. Experimentelle Untersuchungen zur Wirkung von Pressefotos und Fernsehfilmen, Freiburg/München 1987.

Kepplinger, Hans Mathias: Kernenergie. Berichterstattung in ausgewählten Printmedien, Erlangen 1989.

Kepplinger, Hans Mathias: Publizistische Konflikte. Begriffe, Ansätze, Ergebnisse, in: Neidhardt (1994), S. 214-233.

Kepplinger, Hans Mathias (Hrsg.): Angepaßte Außenseiter. Was Journalisten denken und wie sie arbeiten, Freiburg/München 1979.

Kepplinger, Hans Mathias et al.: Instrumentelle Aktualisierung. Grundlagen einer Theorie kognitivaffektiver Medienwirkungen, in: Schulz (1992), S. 161-189.

Kepplinger, Hans Mathias et al.: Am Pranger: Der Fall Späth und der Fall Stolpe, in: Wolfgang Donsbach et al.: Beziehungsspiele - Medien und Politik in der öffentlichen Diskussion. Fallstudien und Analysen, Gütersloh 1993, S. 159-220.

Kepplinger, Hans Mathias / Hans-Bernd Brosius / Norbert Heine: Der Einfluß nonverbaler Verhaltensweisen auf die Personenwahrnehmung, in: Kepplinger (1987), S. 57-91.

Kepplinger, Hans Mathias / Hans-Bernd Brosius / Norbert Heine: Contrast Effects of Nonverbal Behavior in Television Interviews, in: Communications 15/1990, S. 121-134.

Kepplinger, Hans Mathias / Wolfgang Donsbach: Der Einfluß von Kameraperspektiven auf die Auffälligkeit und wahrgenommene Tendenz sowie die Informationsvermittlung und die Personenwahrnehmung, in: Kepplinger (1987), S. 92-124.

Kepplinger, Hans Mathias / Peter Eps / Dirk Augustin: Skandal im Wahlbezirk. Der Einfluß der Presse auf die Wahl des Münchner Oberbürgermeisters 1993, in: Publizistik 40/1995, S. 305-326.

Kepplinger, Hans Mathias / Thomas Hartmann: Die Generalisierung der Personenwahrnehmung 
anhand von Fotos, in: Kepplinger (1987), S. 230-265.

Kepplinger, Hans Mathias / Uwe Hartung: Am Pranger. Eine Fallstudie zur Rationalität öffentlicher Kommunikation, München 1993.

Kepplinger, Hans Mathias / Uwe Hartung: Störfall-Fieber. Wie ein Unfall zum Schlüsselereignis einer Unfallserie wird, Freiburg/München 1995.

Kiesler, Charles A. / Sara B. Kiesler: Role of Forewarning in Persuasive Communications, in Journal of Abnormal and Social Psychology 68/1964, S. 547-549.

Kilz, Hans Werner: Verirrter Journalismus, in: Süddeutsche Zeitung vom 10./11.02.1996.

Kinear, Thomas (Hrsg.): Advances in Consumer Research, Vol. 11, Provo, UT. 1985.

Klapper, Joseph T.: The Effects of Mass Communication, New York 1960.

Klein, Kitty / Debra Holt: The Relationship of Need for Cognition and Media Credibility to Attitudes Toward the Persian Gulf War, in: Contemporary Social Psychology 15/1991, S. 166171.

Klepzig, P.: Zur Entwicklung der Erforschung und Beurteilung der Glaubwürdigkeit, in: Kriminalistik und forensische Wissenschaften 73-74/1989, S. 198-203.

Kline, J. A.: Interaction of Evidence and Readers' Intelligence on the Effects of Short Messages, in: Quarterly Journal of Speech 55/1969, S. 407-413.

Knapp, Mark L. / Mark E. Comadena: Telling It Like It Isn't: A Review of Theory and Research on Deceptive Communications, in: Human Communication Research 5/1979, S. 270-285.

Knapp, Mark L. / R. P. Hart / H. S. Dennis: An Exploration of Deception as a Communication Construct, in: Human Communication Research 1/1974, S. 15-29.

Koch, Ursula E. / Ute Nawratil / Detlef Schröter: Sommer 1789: Französische Revolution und preußische Zeitungsberichte, in: Holger Böning (Hrsg.): Französische Revolution und deutsche Öffentlichkeit, München u.a. 1992, S. 199-249.

Kocher, Douglas J. / Eugene F. Shaw: Newspaper Inaccuracies and Reader Perceptions of Bias, in: Journalism Quarterly 58/1981, S. 471-474, 516.

Köhnken, Günter: Behavioral Correlates of Statement Credibility: Theories, Paradigms, and Results, in: Hermann Wegener / Friedrich Lösel / Jochen Haisch (Hg.): Criminal Behavior and the Justice System. Psychological Perspectives, New York u.a. 1989, S. 271-302.

Köhnken, Günter: Glaubwürdigkeit: Integrative Forschungsperspektiven zu einem vernachlässigten psychologischen Konstrukt, in: Wolfgang Schönpflug (Hrsg.): Bericht über den 36. Kongreß der Deutschen Gesellschaft für Psychologie in Berlin 1988, Göttingen 1988, Band 2, S. 260-275.

Köhnken, Günter: Glaubwürdigkeit. Untersuchungen zu einem psychologischen Konstrukt, München 1990.

Koeske, Gary F. / William D. Crano: The Effect of Congrous and Incongrous Source-Statement Combinations upon the Judged Credibility of a Communication, in: Journal of Experimental Social Psychology 4/1968, S. 384-399. 
Kohn, Paul L. / Suzi Snook: Expectancy-Violation, Similarity, and Unexpected Similarity as Sources of Credibility and Persuasiveness, in: Journal of Psychology 94/1976, S. 185-193.

Kohr, Heinz-Ulrich / Massimo Martini / Angelika Kohr (Hg.): Macht und Bewußtsein. Europäische Beiträge zur Politischen Psychologie, Weinheim 1990.

Kohut, Andrew: Rating the Polls: The Views of Media Elites and the General Public, in: Public Opinion Quarterly 50/1986, S. 1-10.

Koller, Michael: Rebutting Accusations: When Does It Work, When Does It Fail? in: European Journal of Social Psychology 23/1993, S. 373-389.

Krauss, Robert M.: Impression Formation, Impression Management, and Nonverbal Behaviors, in: Higgins/Herman/Zanna (1981), S. 323-341

Kraut, Robert E.: Verbal and Nonverbal Cues in the Perception of Lying, in: Journal of Personality and Social Psychology 36/1978, S. 380-391.

Kraut, Robert : Humans as Lie Detectors. Some Second Thoughts, in: Journal of Communication 30/1980, S. 209-216.

Kröck, Christiane: Das Deutschlandbild in der französischen Tagespresse des Elsaß am Beispiel der 'Dernières Nouvelles d'Alsace', München 1990 (unveröffentlichte Diplomarbeit).

Krüger, Jens / Stephan Ruß-Mohl (Hg.): Risikokommunikation. Technikakzeptanz, Medien und Kommunikationsrisiken, Berlin 1991.

Kruglanski, Arie W.: Attributing Trustworthiness in Supervisor-Worker Relations, in: Journal of Experimental Social Psychology 6/1970, S. 214-232.

Kuhn, Oliver: So belügt uns das Fernsehen, in: Abendzeitung von 19.01.1996.

Kuhn, Oliver / Michael Eder: Ein TV-Held am Ende? Jauch kämpft um seinen guten Ruf, in: Abendzeitung vom 30.01.1996.

Kulp, Daniel H., II: Prestige, as Measured by Single-Experience Changes and Their Permanency, in: Journal of Educational Research 27/1934, S. 663-672.

Landy, David / Harold Sigall: Beauty is Talent: Task Evaluation as a Function of the Performer's Physical Attractiveness, in: Journal of Personality and Social Psychology 29/1974, S. 299304.

La Roche, Walter von: Einführung in den praktischen Journalismus, München ${ }^{12} 1991$.

Lashbrook, William B. / William B. Snavely / Daniel L. Sullivan: The Effects of Source Credibility and Message Information Quantity on the Attitude Change of Apathetics, in: Communication Monographs 44/1977, S. 252-262.

Lawrence, Gary C. / David L. Grey: Subjective Inaccuracies in Local News Reporting, in: Journalism Quarterly 46/1969, S. 753-757.

Lay, C. H. / B. F. Burron: Perception of the Personality of the Hesitant Speaker, in: Perceptual and Motor Skills 26/1968, S. 951-956.

Leavitt, Clark: A Multidimensional Set of Rating Scales For Television Commercials, in: Journal of 
Applied Psychology 34/1970, S. 427-429.

Lee, Raymond S.H.: Credibility of Newspaper and TV News, in: Journalism Quarterly 55/1978, S. 282-287.

Lemert, James B.: Two Studies of Status Conferral, in: Journalism Quarterly 43/1966, S. 25-33, 94.

Lemert, James B.: Components of Source "Image": Hong Kong, Brazil, North America, in: Journalism Quarterly 46/1969, S. 306-313, 418.

Lemert, James B.: News Media Competition Under Conditions Favorable to Newspapers, in: Journalism Quarterly 47/1970, S. 272-280.

Lemert, James B. / Karl J. Nestvold: Television News and Status Conferral, in: Journal of Broadcasting 14/1970, S. 491-497.

Levine, John M. / Ronald S. Valle: The Convert as a Credible Communicator, in: Social Behaviour and Personality, 3/1975, S. 81-90.

Lewis, Helen B.: Studies in the Principles of Judgments and Attitudes: IV. The Operation of 'Prestige Suggestion', in: Journal of Social Psychology 14/1941, S. 229-256.

Lewis, James J.: A Criticism of "The Factor Structure of Source Credibility as a Function of the Speaking Situation, in: Speech Monographs 41/1974, S. 287-290.

Lichtenberg, Judith / Douglas MacLean: The Role of Media in Risk Kommunication, in: Jungermann / Kasperson / Wiedemann (1988), S. 33-48.

Lilienthal, Volker: Wickerts Seitensprung, in: Journalist 12/1995, S. 43.

Lilienthal, Volker: Riskantes Tingeln, in: Journalist 7/1995, S. 32-35.

Lindzey, Gardner / Elliot Aronson (Hg.): The Handbook of Social Psychology. Second Edition, Vol. 3, Reading, Mass. 1969.

Linz, Daniel G. / Steven Penrod: Increasing Attorney Persuasiveness in the Courtroom, in: Law and Psychology Review 8/1984, S. 1-47.

Lirtzman, Sidney I. / Avichai Shuv-Ami: Credibility of Sources of Communication on Products' Safety Hazards, in: Psychological Reports 58/1986, S. 707-718.

Liska, Jo: Situational and Topical Variations in Credibility Criteria, in: Communication Monographs 45/1978, S. 85-92.

Littlejohn, Stephen W.: A Bibliography of Studies Related to Variables of Source Credibility, in: Bibliographic Annual in Speech Communication 2/1971, S. 1-40.

Littlepage, G. E. / M. A. Pineault: Verbal, Facial and Paralinguistic Cues to the Detection of Truth and Lying, in: Personality and Social Psychology Bulletin 4/1978, S. 461-464.

Littlepage, G. E. / M. A. Pineault: Detection of Deceptive Factual Statements from the Body and the Face, in: Personality and Social Psychology Bulletin 5/1979, S. 325-328.

Littmann, Eckhard / Hans Szewiczyk: Zu einigen Kriterien und Ergebnissen forensisch-psychologischer Glaubwürdigkeitsbegutachtung von sexuell mißbrauchten Kindern und Jugendlichen, in: Forensia 4/1983, S. 55-72. 
Lohisse, Jean: Communication et developpement, in: Communications 3/1977, S. 47-54.

Loken, Barbara / Beth Howard-Pitney: Effectiveness of Cigarette Advertisements on Women: An Experimental Study, in: Journal of Applied Psychology 73/1988, S. 378-382.

Lorge, Irving: Prestige, Suggestion, and Attitudes, in: Journal of Social Psychology 7/1936, S. 386402.

Lowry, Dennis T.: Demographic Similarity, Attitudinal Similarity, and Attitude Change, in: Public Opinion Quarterly 37/1973, S. 192-208.

Ludlum, Thomas S.: Effects of Certain Techniques of Credibility upon Audience Attitude, in: Speech Monographs 25/1958, S. 278-284.

Luhmann, Niklas: Vertrauen. Ein Mechanismus der Reduktion sozialer Komplexität, Stuttgart ${ }^{3} 1989$.

Lui, Louisa / Lionel Standing: Communicator Credibility: Trustworthiness Defeats Expertness, in: Social Behavior and Personality 17/1989, S. 219-221.

Lukesch, Helmut / Karl-Heinz Kischkel / Wolfgang Nöldner: Zur Beurteilung von Informationsquellen und Medien im Zusammenhang mit dem Reaktorunfall von Tschernobyl, in: Publizistik 32/1987, S. 154-158.

Lumsden, Donald L.: An Experimental Study of Source-Message Interaction in a Personality Impression Task, in: Communication Monographs 44/1977, S. 121-129.

Lurie, Walter A.: Measurement of Prestige and Prestige-Suggestibility, in: Journal of Social Psychology 9/1938, S. 219-225.

Lykken, D. T.: Psychology and the Lie Detector Industry, in: American Psychologist 29/1974, S. 725-739.

MacKenzie, Scott B. / Richard J. Lutz: An Empirical Examination of the Structural Antecedents of Attitude Toward the Ad in an Advertising Pretesting Context, in: Journal of Marketing 53/1989, S. 48-65.

Macrae, C. Neil / John W. Shepherd / Alan B. Milne: The Effects of Source Credibility on the Dilution of Stereotype-Based Judgments, in: Personality and Social Psychology Bulletin 18/1992, S. 765-775.

Maddux, James E. / Ronald W. Rogers: Effects of Source Expertness, Physical Attractiveness, and Supporting Arguments on Persuasion: A Case of Brains over Beauty, in: Journal of Personality and Social Psychology 39/1980, S. 235-244.

Maier, Norman R. F.: Sensivity to Attempts at Deception in an Interview, in: Personnel Psychology 19/1965, S. 55-66.

Maier, Norman R. F. / J. Janzen: The Reliability of Persons Making Judgments of Honesty and Dishonesty, in: Perceptual and Motor Skills 25/1967, S. 141-151.

Maier, Norman R. F. / James A. Thurber: Accuracy of Judgments of Deception When an Interview is Watched, Heard, and Read, in: Personnel Psychology 21/1968, S. 23-30. 
Maier, R. A. / P. J. Lavrakas: Lying Behavior and Evaluation of Lies, in: Perceptual and Motor Skills 42/1976, S. 575-581.

Maile, Carlton A. / A. H. Kizilbash: Derogation of Source: A Matter of Definition, in: Psychological Reports 39/1976, S. 780-782.

Maletzke, Gerhard: Psychologie der Massenkommunikation. Theorie und Systematik, Hamburg 1963.

Mandell, Lee M. / Donald L. Shaw: Judging People in the News - Unconsciously: Effect of Camera Angle and Bodily Activity, in: Journal of Broadcasting 17/1973, S. 353-362.

Manstead, A. / C. McCulloch: Sex-Role Stereotyping in British Television Advertisements, in: British Journal of Social Psychology 20/1981, S. 171-180.

Markham, David: The Dimensions of Source Credibility of Television Newsmasters, in: Journal of Communication 18/1968, S. 57-64.

Mayton, Daniel M. / Elizabeth A. Nagel / Reese Parker: The Perceived Effects of Drug Messages on Use Patterns in Adolescents, in: Journal of Drug Education 20/1990, S. 305-318.

McCain, Thomas A. / Joseph Chilberg / Jacob Wakshlag: The Effect of Camera Angle on Source Credibility and Attraction, in: Journal of Broadcasting 21/1977, S. 35-46.

McCallum, David B. / Sharon Lee Hammond / Vincent T. Covello: Communicating about Environmental Risks: How the Public Uses and Perceives Information Sources, in: Health Education Quarterly 18/1991, S. 349-361.

McClintock, Charles C. / Raymond G. Hunt: Nonverbal Indicators of Affect and Deception in an Interview Setting, in: Journal of Applied Social Psychology 5/1975, S. 54-67

McCornack, Steven A. / Malcolm R. Parks: Deception Detection and Relationship Development and Relationship Development: The Other Side of Trust, in: Margaret L. McLaughlin Hrsg.): Communication Yearbook 9, Beverly Hills /London / New Delhi 1986, S. 377-389.

McCroskey, James C.: Scales for the Measurement of Ethos, in: Speech Monographs 33/1966, S. 65-72.

McCroskey, James C.: A Summary of Experimental Research on the Effects of Evidence in Persuasive Communication, in: Quarterly Journal of Speech 55/1969, S. 169-176.

McCroskey, James C.: The Effects of Evidence as an Inhibitor of Counterpersuasion, in: Speech Monographs 37/1970, S. 188-194.

McCroskey, James C. / Walter H. Combs: The Effects of the Use of Analogy on Attitude Change and Credibility, in: Journal of Communication 19/1969, S. 333-339.

McCroskey James C. / Robert E. Dunham: Ethos: A Confounding Element in Communication Research, in: Speech Monographs 33/1966, S. 456-463.

McCroskey, James C. / Paul R. Hamilton / Allen N. Weiner: The Effect of Interaction Behavior on Source Credibility, Homophily, and Interpersonal Attraction, in: Human Communication Research 1/1974, S. 42-52. 
McCroskey, James C. / Thomas A. Jenson: Image of Mass Media News Sources, in: Journal of Broadcasting 19/1975, S. 169-180.

McCroskey, James C. / Thomas A. McCain: The Measurement of Interpersonal Attraction, in: Speech Monographs 41/1974, S. 261-266.

McCroskey, James C. / R. Samuel Mehrley: The Effects of Disorganization and Nonfluency on Attitude Change and Source Credibility, in: Speech Monographs 36/1969, S. 13-21.

McDaniel, William F. / Leslie C. Vestal: Issue Relevance and Source Credibility as a Determinant of Retention, in: Bulletin of the Psychonomic Society 5/1975, S. 481-482.

McEwen, William J. / Bradley S. Greenberg: The Effects of Message Intensity on Receiver Evaluations of Source, Message and Topic, in: Journal of Communication 20/1970, S. 340350.

McGarry, James / Clyde Hendrick: Communicator Credibility and Persuasion, in: Memory \& Cognition 2/1974, S. 82-86.

McGinnies, Elliott: Studies in Persuasion: IV. Source Credibility and Involvement as Factors in Persuasion with Students in Taiwan, in: Journal of Social Psychology 74/1968, S. 171-180.

McGinnies, Elliott: Initial Attitude, Source Credibility, and Involvement as Factors in Persuasion, in: Journal of Experimental Social Psychology 9/1973, S. 285-296.

McGinnies, Elliott / Charles D. Ward: Persuasibility as a Function of Source Credibility and Locus of Control: Five Cross Cultural Experiments, in: Journal of Personality 42/1974, S. 360371.

McGinnies, Elliott / Charles D. Ward: Better Liked Than Right: Trustworthiness and Expertise as Factors in Credibility, in: Personality and Social Psychology Bulletin 6 (3)/1980, S. 467472.

McGuire, William J.: The Nature of Attitudes and Attitude Change, in: Gardner Lindzey / Elliot Aronson (Hg.): The Handbook of Social Psychology. Second Edition, Vol. 3, Reading, Mass. 1969, S. 136-314.

McGuire, William J. / Demetrios Papageorgis: Effectiveness of Forewarning in Developing Resistance to Persuasion, in: Public Opinion Quarterly 26/1962, S. 24-34.

McKillip, Jack: Credibility and Impression Formation, in: Personality and Social Psychology Bulletin 1/1975, S. 521-524.

McKillip, Jack / John D. Edwards: Source Characteristics and Attitude Change, in: Personality and Social Psychology Bulletin 1/1975, S. 135-137.

McLaughlin, Margaret: Recovering the Structure of Credibility Judgments: An Alternative to Factor Analysis, in: Speech Monographs 42/1975, S. 221-228.

McPeek, R. W. / J. D. Edwards: Expectancy Disconfirmation and Attitude Change, in: Journal of Social Psychology 96/1975, S. 193-208.

Mehrabian, A.: Nonverbal Betraying of Feeling, in: Journal of Experimental Research in Personality 5/1975, S. 64-73. 
Mehrley, R. Samuel / James C. McCroskey: Opinionated Statements and Attitude Intensity as Predictors of Attitude Change and Source Credibility, in: Speech Monographs 37/1970, S. 47-52.

Mellinger, Glen D.: Interpersonal Trust as a Factor in Communication, in: Journal of Abnormal and Social Psychology 52/1956, S. 304-309.

Merten, Klaus: Risikokommunikation - Probleme und Perspektiven, in: Peter C. Compes (Hrsg.): Risiko - subjektiv und objektiv, Wuppertal 1989, S. 17-49.

Merten, Klaus / Siegfried J. Schmidt / S. Weischenberg (Hg.): Die Wirklichkeit der Medien. Eine Einführung in die Kommunikationswissenschaft, Opladen 1994.

Meyer, Philip: Elitism and Newspaper Believability, in: Journalism Quarterly 50/1973, S. 31-36.

Meyer, Philip: Defining and Measuring Credibility Of Newspapers: Developing an Index, in: Journalism Quarterly 65/1988, S. 567-574, 588.

Meyer, Timothy J.: Media Credibility: The State of the Research, in: Public Telecommunications Review 2 (4)/1974, S. 48-52.

Meyer, Timothy P. / Anne Hexamer: Perceived Truth and Trust in Television Advertising Among Mexican-American Adolescents: Socialization and Developmental Considerations, in: Journal of Broadcasting 25/1981, S. 139-153.

Meyer, Werner: Zeitungspraktikum, Percha 1983.

Michael, William / Bernard Rosenthal / Michael DeCamp: An Experimental Investigation of Prestige-Suggestion for Two Types of Literary Material, in: Journal of Psychology 28/1949, S. 303-323.

Mika, Stanislaw: Some Determinants of Source Credibility, in: Polish Psychological Bulletin 12/1981, S. 79-86.

Milbourn, M. Thomas / Vernon A. Stone: Source-Message Orientation and Components of Source Credibility, in: Journalism Quarterly 49/1972, S. 663-668.

Miller, Arthur G.: Role of Physical Attractiveness in Impression Formation, in: Psychonomic Science 19/1970, S. 241-243.

Miller, Arthur G.: Social Perception of Internal-External Control, in: Perceptual and Motor Skills 30/1970, S. 103-110.

Miller, Dale T. / Ronald A. Hoppe: The Effect of Regional Similarity-Dissimilarity on Communicator Credibility, in: Language and Speech 16/1973, S. 211-217.

Miller, Gerald R.: Communication and Persuasion Research: Current Problems and Prospects, in: Quarterly Journal of Speech 54/1968, S. 268-276.

Miller, Gerald R.: Persuasion, in: C. R. Berger / S. H. Chaffee (Hg.): Handbook of Communication Science, Newbury Park, CA. 1987, S. 446-483.

Miller, Gerald R. / John Baseheart: Source Trustworthiness, Opinionated Statements, and Response to Persuasive Communication, in: Speech Monographs 36/1969, S. 1-7. 
Miller, Gerald R. / Murray A. Hewgill: The Effect of Variations in Nonfluency on Audience Ratings of Source Credibility, in: Quarterly Journal of Speech 50/1964, S. 36-44.

Miller, Gerald R. / Michael McReynolds: Male Chauvinism and Source Competence: A Research Note, in: Speech Monographs 40/1973, S. 154-155.

Miller, Gerald R. et al.: "...and nothing but the truth": How well can Observers Detect Deceptive Testimony? In: B. D. Sales (Hrsg.): Perspectives in Law and Psychology, Vol. II, The Jury, Judicial and Trial Process, New York 1981, S. 145-179.

Miller, M. Mark / Michael W. Singletary / Shu-Ling Chen: The Roper Question and Television vs. Newspapers as Sources of News, in: Journalism Quarterly 65/1988, S. 12-19.

Miller, Norman et al.: Speed of Speech and Persuasion, in: Journal of Personality and Social Psychology 34/1976, S. 615-625.

Milliman, Ronald E. / Robert C. Erffmeyer: Improving Advertising Aimed at Seniors, in: Journal of Advertising Research 29/1989-90, S. 31-36.

Mills, Judson: Opinion Change as a Function of the Communicator's Desire to Influence and Liking for the Audience, in: Journal of Experimental Social Psychology 2/1966, S. 152-159.

Mills, Judson / Elliot Aronson: Opinion Change as a Function of the Communicator's Attractiveness and Desire to Influence, in: Journal of Personality and Social Psychology 1/1965, S. 173177.

Mills, Judson / John Harvey: Opinion Change as a Function of When Information about the Communicator is Received and Whether He Is Attractive or Expert, in: Journal of Personality and Social Psychology 21/1972, S. 52-55.

Mills, Judson / Jerald M. Jellison: Effect on Opinion Change of Similarity between the Communicator and the Audience He Adressed, in: Journal of Personality and Social Psychology 9/1968, S. 153-156.

Mills, Judson / Charles E. Kimble: Opinion Change as a Function of Perceived Similarity of the Communicator and Subjectivity of the Issue, in: Bulletin of the Psychonomic Society 2/1973, S. 35-36.

Mischke, Eckhard: "Flüsterjets und Donnervögel". Imageanalyse am Beispiel der Berichterstattung über den Flughafen München 2, München 1989 (unveröffentlichte Magisterarbeit).

Molotch, Harvey / Marilyn Lester: News as Purposive Behavior: On the Strategic Use of Routine Events, Accidents, and Scandals, in: American Sociological Review 39/1974, S. 101-112.

Mondak, Jeffery J.: Perceived Legitimacy of Supreme Court Decisions: Three Functions of Source Credibility, in: Political Behavior 12/1990, S. 363-384.

Moodie, Graeme C.: On Political Scandal and Corruption, in: Government and Opposition 15(2)/1980, S. 208-222.

Moore, Henry T.: The Comparative Influence of Majority and Expert Opinion, in: American Journal of Psychology 32/1921, S. 16-20. 
Moser, Helmut (Hrsg.): L' Eclat c'est moi. Zur Faszination unserer Skandale, Weinheim 1989.

Mosier, Nancy R. / Andrew Ahlgren: Credibility of Precision Journalism, in: Journalism Quarterly 58/1981, S. 375-381, 518.

Muckenhaupt, Manfred: Die neue Qualität des Mediensports. Auswirkungen der Medienkonkurrenz auf die Sportberichterstattung des Fernsehens, in: Brennpunkte der Sportwissenschaft 4/1990, S. 5-19.

Mulac, Anthony / A. Robert Sherman: Relationships among Four Parameters of Speaker Evaluation: Speech Skill, Source Credibility, Subjective Speech Anxiety, and Behavioral Speech Anxiety, in: Speech Monographs 42/1975, S. 302-310.

Mulder, Ronald: Media Credibility: A Use-Gratifications Approach, in: Journalism Quarterly 57/1980, S. 474-477.

Myers, Michele Tolela / Alvin A. Goldberg: Group Credibility and Opinion Change, in: Journal of Communication 20/1970, S. 174-179.

Nail, Richard L. / Eric Gunderson: The Youthful Drug Abuser and Drug Abuse Education - Closing the Credibility Gap, in: Journal of Drug Education 5/1975, S. 65-75.

Nawratil, Ute: "Wenig Lärm um viel". Die Berichterstattung über Reproduktionsmedizin und Genforschung. Vergleichende Inhaltsanalyse, München 1987 (unveröffentlichte Magisterarbeit).

Neckel, Sieghard: Das Stellhölzchen der Macht. Zur Soziologie des politischen Skandals, in: Leviathan 14/1986, S. 581-605.

Neckel Sieghard: Machen Skandale apathisch? In: Ebbinghausen / Neckel (1989), S. 234-257.

Neidhardt, Friedhelm (Hrsg.): Öffentlichkeit, Öffentliche Meinung, soziale Bewegungen, Opladen 1994 (=Sonderheft 34 der Kölner Zeitschrift für Soziologie und Sozialpsychologie).

Newcomb, Theodore M.: The Prediction of Interpersonal Attraction, in: Carl W. Backman / Paul F. Secord (Hg.): Problems in Social Psychology. Selected Readings, New York u.a. 1966, S. 168-179.

Newhagen, John / Clifford Nass: Differential Criteria for Evaluating Credibility of Newspapers and TV News, in: Journalism Quarterly 66/1989, S. 277-284.

Niketta, Reiner: Das Stereotyp der physischen Attraktivität, in: Hassebrauck / Niketta (1993), S. 163-200.

Noblé, Heidrun: Grau in grau, Herr Niemetz! In: Die Woche vom 27.10.1995.

Noelle-Neumann, Elisabeth: Die Entfremdung. Brief an die Zeitschrift 'Journalist', in: Hans Mathias Kepplinger (Hrsg.): Angepaßte Außenseiter. Was Journalisten denken und wie sie arbeiten, Freiburg/München 1979, S. 261-280.

Norman, Ross: When What is Said is Important: A Comparison of Expert and Attractive Sources, in: Journal of Experimental Social Psychology 12/1976, 294-300.

Norris, Eleanor L.: Attitude Change as a Function of Open or Closed-Mindedness, in: Journalism Quarterly 42/1965, S. 571-575. 
Ohanian, Roobina: Construction and Validation of a Scale to Measure Celebrity Endorsers Perceived Expertise, Trustworthiness, and Attractiveness, in: Journal of Advertising 19/1990, S. 3952.

Ohanian, Roobina: The Impact of Celebrity Spokespersons' Perceived Image on Consumers' Intention to Purchase, in: Journal of Advertising Research 31/1991, S. 46-54.

O'Keefe, Daniel J.: The Persuasive Effects of Delaying Identification on High- and Low-Credibility Communicators: A Meta-Analytic Review, in: Central States Speech Journal 38/1987, S. 63-72.

Olson, James M. / A. Victoria Cal: Source Credibility, Attitudes, and the Recall of Past Behaviours, in: European Journal of Social Psychology 14/1984, S. 203-210.

O'Neal, Gwendolyn S. / Mary Lapitsky: Effects of Clothing as Nonverbal Communication on Credibility of the Message Source, in: Clothing and Textiles Research Journal 9/1991, S. 28-34.

O'Reilly, Charles A. III / Karlene H. Roberts: Relationships Among Components of Credibility and Communication Behaviors in Work Units, in: Journal of Applied Psychology 61/1976, S. 99-102.

Osgood, Charles E. / George J. Suci / Percy H. Tannenbaum: The Measurement of Meaning, Urbana 1957.

Ostermeier, Terry H.: Effects of Type and Frequency of Reference Upon Perceived Source Credibility and Attitude Change, in: Speech Monographs 34/1967, S. 137-144.

o.V.: Werbeauftritte überzeugen nicht, in: Journalist 2/1996, S. 10.

o.V.: Brehmer über TV-Frauen, in: Süddeutsche Zeitung vom 21./22.10.1995.

o.V.: "Ich bedauere den Vorfall", in: Süddeutsche Zeitung vom 14.11.1995.

o.V.: "Der Vetter aus Dingsda" in: Spiegel Nr. 53 vom 28.12.1992.

o.V.: "Tarnen, tricksen, täuschen", in: Spiegel Nr. 1 vom 04.01.1993.

o.V.: Alle wollen eine Krause-Putzfrau. Für 2,10 Mark und der Staat zahlt den Rest, in: Bild vom 23.03.1993.

o.V.: Treu und Glauben. Was die Deutschen von Presse, Funk und Fernsehen halten, in: Spiegel special 1/1995, S. 168.

o.V.: Kaum Glauben an TV-Schicksale, in: Kölner Stadt-Anzeiger vom 5. Mai 1997.

Page, Monte M.: Role of Demand Awareness in the Communicator Credibility Effect, in: Journal of Social Psychology 82/1970, S. 57-66.

Paletz, David L. et al.: Polls in the Media: Content, Credibility, and Consequences, in: Public Opinion Quarterly 44/1980, S. 495-513.

Pallak, Suzanne R.: Salience of Communicator's Physical Attractiveness and Persuasion: A Heuristic versus Systematic Processing Interpretation, in: Social Cognition 2/1983, S. 158-170. 
Pallak, Suzanne R. / Enid Murroni / Juliann Koch: Communicator Attractiveness and Expertise, Emotional versus Rational Appeals, and Persuasion: A Heuristic versus Systematic Processing Interpretation, in: Social Cognition 2/1983, S. 122-141.

Patzer, Gordon L.: Source Credibility as a Function of Communicator Physical Attractiveness, in: Journal of Business Research 11/1983, S. 229-241.

Patzer, Gordon L.: The Physical Attractiveness Phenomena, New York 1985.

Paulson, Stanley F.: The Effects of the Prestige of the Speaker and Acknowledgement of Opposing Arguments on Audience Retention and Shift of Opinion, in: Speech Monographs 21/1954, S. 267-271.

Pearce, W. Barnett: Trust in Interpersonal Communication, in: Speech Monographs 41/1974, S. 236244.

Pearce, W. Barnett / Bernard J. Brommel: Vocalic Communication and Persuasion, in: Quarterly Journal of Speech 58/1972, S. 293-306.

Pearce, W. Barnett / Forrest Conklin: Nonverbal Vocalic Communication and Perceptions of a Speaker, in: Speech Monographs 38/1971, S. 235-241.

Peck, Teresa: When Women Evaluate Women, Nothing Succeeds Like Success: The Differential Effects of Status Upon Evaluations of Male and Female Professional Ability, in: Sex Roles 4/1978, S. 205-213.

Percy, Larry / John R. Rossiter: Advertising Stimulus Effects: a Review, in: Planung und Analyse 910/1991, S. 340-351.

Perez, Juan Antonio / Gabriel Mugny: Changement d'attitude, crédibilité et influence minoritaire: interdépendance et indépendance de la comparaison sociale, in: Schweizerische Zeitschrift für Psychologie 49/1990, S. 150.158.

Perger, Werner A.: Unter Fälschern, in: Die Zeit vom 02.02.1996.

Perloff, Richard M.: The Dynamics of Persuasion, Hilsdale N.J. 1993.

Petermann, Franz: Psychologie des Vertrauens, München ${ }^{2} 1992$.

Peters, Hans Peter: The Credibility of Information Sources in West Germany after the Chernobyl Disaster, in: Public Understanding of Science 1/1992, S. 325-343.

Peters, Hans Peter: Risikokommunikation in den Medien, in: Merten / Schmidt / Weischenberg (1994), S. 329-351.

Peters, Hans Peter: Wissenschaftliche Experten in der öffentlichen Kommunikation über Technik, Umwelt und Risiken, in: Friedhelm Neidhardt (Hrsg.): Öffentlichkeit, Öffentliche Meinung, soziale Bewegungen, Opladen 1994 (=Sonderheft 34 der Kölner Zeitschrift für Soziologie und Sozialpsychologie), S. 162-190.

Peters, Hans Peter et al.: Die Reaktionen der Bevölkerung auf die Ereignisse in Tschernobyl. Ergebnisse einer Befragung, in: Kölner Zeitschrift für Soziologie und Sozialpsychologie 39/1987, S. 764-782. 
Peters, Hans Peter / Leo Hennen: Orientierung unter Unsicherheit. Bewertung der Informationspolitik und Medienberichterstattung nach 'Tschernobyl', in: Kölner Zeitschrift für Soziologie und Sozialpsychologie 42/1990, S. 300-312.

Petty, Richard E. / John T. Cacioppo: Effects of Forewarning on Persuasive Intent and Involvement on Cognitive Responses and Persuasion, in: Personality and Social Psychology Bulletin 5/1979, S. 173-176. [1979a]

Petty, Richard E. / John T. Cacioppo: Issue Involvement Can Increase or Decrease Persuasion by Enhancing Message-Relevant Cognitive Responses, in: Journal of Personality and Social Psychology 37/1979, S. 1915-1926. [1979b]

Petty, Richard E. / John T. Cacioppo: The Effects of Involvement on Responses to Argument Quantity and Quality: Central and Periphal Routes to Persuasion, in: Journal of Personality and Social Psychology 46/1984, S. 69-81.

Petty, Richard E. / John T. Cacioppo: Source Factors and the Elaboration Likelihood Model of Persuasion, in: Thomas Kinear (Hrsg.): Advances in Consumer Research, Vol. 11, Provo, UT. 1985, S. 668-672.

Petty, Richard E. / John T. Cacioppo: Communication and Persuasion, New York 1986. [1986a]

Petty, Richard E. / John T. Cacioppo: The Elaboration Likelihood Model of Persuasion, in: Leonard Berkowitz (Hrsg.): Advances in Experimental Social Psychology, Vol. 19, New York/London 1986, S. 123-205. [1986b]

Petty, Richard E. / John T. Cacioppo: Involvement and Persuasion: Tradition versus Integration, in: Psychological Bulletin 107/1990, S. 367-374.

Petty, Richard E. / John T. Cacioppo / Rachel Goldman: Personal Involvement as a Determinant of Argument-Based Persuasion, in: Journal of Personality and Social Psychology 41/1981, S. 847-855.

Petty, Richard E. / John T. Cacioppo / David Schumann: Central and Peripheral Routes to Advertising Effectiveness: The Moderating Role of Involvement, in: Journal of Consumer Research 10/1983, S. 135-146.

Pippig, Gerhard: Verwaltungsskandale. Zur Korruption in der öffentlichen Verwaltung, in: Aus Politik und Zeitgeschichte (Beilage zu 'Das Parlament') B7/1990, S. 11-20.

Plax, Timothy G. / Lawrence B. Rosenfeld: Individual Differences in the Credibility and Attitude Change Relationship, in: Journal of Social Psychology 111/1980, S. 79-89.

Poitsch, Christian: Die Parteien vor der Wahl. Stereotypenuntersuchung zur Berichterstattung vor der Bundestagswahl 1987. Inhaltsanalyse, München 1988 (unveröffentlichte Magisterarbeit).

Powell, Frederic A.: Open- and Closed-Mindedness and the Ability to Differentiate Source and Message, in: Journal of Abnormal and Social Psychology 65/1962, S. 61-64.

Powell, Frederic A.: Source Credibility and Behavioral Compliance as Determinants of Attitude 
Change, in: Journal of Personality and Social Psychology 2/1965, S. 669-676.

Powell, Frederic A. / Gerald R. Miller: Social Approval and Disapproval in Anxiety-Arousing Communications, in: Speech Monographs 34/1967, S. 152-159.

Powell, F. C. / J[ohn] W. Wanzenried: Perceptual Changes in Source Credibility: Repeated Tests Using Two Candidates during a Political Campaign, in: Perceptual and Motor Skills 73/1991, S. 1107-1114.

Powell, Larry et al.: Smoking Artefacts: Factors of Source Evaluation, in: Perceptual and Motor Skills 47/1978, S. 933-934.

Pratkanis, Anthony R. et al.: In Search of Reliable Persuasion Effects: III. The Sleeper Effect is Dead. Long Live the Sleeper Effect, in: Journal of Personality and Social Psychology 54/1988, S. 203-218.

Pratt, Cornelius: How Media Credibility Ratings of African and U.S. Students Compare, in: Journalism Quarterly 59/1982, S. 581-587.

Preiser, Siegfried: Ganz normale menschliche Reaktionen. Skandalverarbeitung im Spannungsfeld politischer Erfahrungen, Werte und Einstellungen, in: Moser (1989), S. 98-117.

Pscherer, Jörg: Glaubwürdigkeit politischer Akteure. Eine empirische Analyse am Beispiel der Spitzenkandidaten zur Bundestagswahl 1990, in: Zeitschrift für Politische Psychologie 1/1994, S. 73-79.

Pürer, Heinz (Hrsg.): Medienereignisse - Medienwirkungen? Salzburg 1985.

Reagan, Joey / Jayne Zenaty: Local News Credibility: Newspapers vs. TV Revisited, in: Journalism Quarterly 56/1979, S. 168-172.

Regionalpresse e.V.: Einkaufs- und Informationsverhalten 1990. Die Medienleistung von Presse, Hörfunk und Fernsehen im Vergleich, hrsg. von gzm-Gesellschaft für Zeitungsmarketing mbH, im Auftrag der Regionalpresse e.V., Frankfurt 1991.

Reinard, John C.: The Empirical Study of the Persuasive Effects of Evidence. The Status After Fifty Years of Research, in: Human Communication Research 15/1988, S. 3-59.

Reinsch, N. Lamar, Jr.: An Investigation of the Effects of the Metaphor and Simile in Persuasive Discourse, in: Speech Monographs 38/1971, S. 142-145.

Reinsch, N. L.[amar], Jr.: Figurative Language and Source Credibility: A Preliminary Investigation and Reconceptualization, in: Human Communication Research 1/1974, S. 75-80.

Renn, Ortwin: Public Responses to the Chernobyl Accident, in: Journal of Environmental Psychology 10/1990, S. 151-167.

Renn, Ortwin / Debra Levine: Credibility and Trust in Risk Communication, in: Jungermann / Kasperson / Wiedemann (1988), S. 51-81.

Rhine, Ramon J. / Robert M. Kaplan: The Effect of Incredulity upon Evaluation of the Source of a Communication, in: Journal of Social Psychology 88/1972, S. 255-266.

Rhine, Ramon J. / Laurence J. Severance: Ego-Involvement, Discrepancy, Source Credibility, and 
Attitude Change, in: Journal of Personality and Social Psychology 16/1970, S. 175-190.

Rieke, Richard R. / Malcolm O. Sillars: Argumentation and the Decision Making Process, New York u.a. 1975.

Riehl-Heyse, Herbert: Das nützliche Ärgernis. Warum wir Skandale brauchen, in: Süddeutsche Zeitung vom 10./11. Juli 1982.

e,Riffe, Daniel: Relative Credibility Revisited: How 18 Unnamed Sources Are Rated, in: Journalism Quarterly 57/1980, S. 618-623.

Rimmer, Tony / David Weaver: Different Questions, Different Answers? Media Use and Media Credibility, in: Journalism Quarterly 64/1987, S. 28-36, 44.

Ripley, William K.: Medium of Presentation: Does It Make a Difference in the Reception of Evaluation Information? in: Educational Evaluation and Policy Analysis 7/1985, S. 417425.

Ritchie, Elaine / E. J. Phares: Attitude Change as a Function of Internal-External Control and Communicator Status, in: Journal of Personality 37/1969, S. 429-443.

Robinson, Michael D. / Joel T. Johnson / Stephanie A. Shields: On the Advantages of Modesty: The Benefits of a Balanced Self-Presentation, in: Communication Research 22/1995, S. 575591.

Robinson, Michael J. / Andrew Kohut: Believability and the Press, in: Public Opinion Quarterly 52/1988, S. 174-189.

Rogers, Everett M. / Dilip K. Bhowmik: Homophily-Heterophily: Relational Concepts for Communication Research, in: Public Opinion Quarterly 34/1970, S. 523-538.

Rokeach, Milton: The Open and Closed Mind, New York 1960.

Roloff, M. E. / G. R. Miller (Hg.): Persuasion: New Directions in Theory and Research, Newbury Park, CA. 1980.

Roper, Burns W.: Changing Public Attitudes toward Television and Other Media 1959-1976, in: Communications 4/1978, S. 220-238.

Roper, Burns W.: Evaluating Polls with Poll Data, in: Public Opinion Quarterly 50/1986, S. 10-16.

Rosenbaum, Milton E. / Irwin P. Lewin: Impression Formation as a Function of Source Credibility and Order of Presentation of Contradictory Information, in: Journal of Personality and Social Psychology 10/1968, S. 167-174.

Rosenbaum, Milton E. / Irwin P. Lewin: Impression Formation as a Function of Source Credibility and the Polarity of Information, in: Journal of Personality and Social Psychology 12/1969, S. 34-37.

Rosenthal, Paul I.: The Concept of Ethos and the Structure of Persuasion, in: Speech Monographs 33/1966, S. 114-126.

Rosenthal, Paul I.: The Concept of the Paramessage in Persuasive Communication, in: Quarterly Journal of Speech 58/1972, S. 15-30. 
Rosenthal, Robert / Bella M. DePaulo: Sex Differences in Eavesdropping on Nonverbal Cues, in: Journal of Personality and Social Psychology 37/1979, S. 273-285.

Roth, Roland: Eine korrupte Republik? Konturen politischer Korruption in der Bundesrepublik, in: Ebbinghausen / Neckel (1989), S. 201-233.

Rotter, Julian B.: General Expectancies for Internal versus External Control of Reinforcement, in: Psychological Monographs 80/1966, S. 1-28.

Rotter, Julian B.: A New Scale for the Measurement of Interpersonal Trust, in: Journal of Personality 35/1967, S. 651-665.

Rotter, Julian B.: Generalized Expectancies for Interpersonal Trust, in: American Psychologist 26/1971, S. 443-452.

Rotter, Julian B. / Donald K. Stein: Public Attitudes toward Trustworthiness, Competence and Altruism of Twenty Selected Occupations, in: Journal of Applied Social Psychology 1/1971, S. 334-344

Rotton, James / Brian F. Blake / Richard Heslin: Dogmatism, Trust, and Message Acceptance, in: Journal of Psychology 96/1977, S. 81-88.

Ruff, Frank M.: Risikokommunikation als Aufgabe für die Umweltmedizin, in: Karl Aurand / Barbara P. Hazard / Felix Tretter (Hg.): Umweltbelastungen und Ängste. Erkennen. Bewerten. Vermeiden, Opladen 1993, S. 327-364.

Ruhrmann, Georg: Risikokomunikation, in: Publizistik 37/1992, S. 5-24.

Ruß-Mohl, Stephan: Die Nebenchefin, in: MediumMagazin 10/1995, S. 70-72.

Ryan, Michael: News Content, Geographical Origin and Perceived Media Credibility, in: Journalism Quarterly 50/1973, S. 312-318.

Saadi, Mitchel / Paul R. Farnsworth: The Degrees of Acceptance of Dogmatic Statements and Preferences for Their Supposed Markers, in: Journal of Abnormal and Social Psychology 29/1934-35, S. 143-150.

Salmon, Charles T. / Jung-Sook Lee: Perceptions of Newspaper Fairness: A Structural Approach, in: Journalism Quarterly 60/1983, S. 663-670.

Salter, Marty M. / Deborah Weider-Hatfield / Donald L. Rubin: Generic Pronoun Use and Perceived Speaker Credibility, in: Communication Quarterly 31/1983, S. 180-184.

Salwen, Michael B.: Credibility of Newspaper Opinion Polls: Source, Source Intent and Precision, in: Journalism Quarterly 64/1987, S. 813-819.

Sargent, Leslie W.: Communicator Image and News Reception, in: Journalism Quarterly 42/1965, S. $35-42$.

Sawyer, Thomas M. Jr.: Shift of Attitude Following Persuasion as Related to Estimate of Majority Attitude, in: Speech Monographs 22/1955, S. 68-78.

Scanlon, T. Joseph: A New Approach to Study of Newspaper Accuracy, in: Journalism Quarterly 49/1972, S. 587-590.

Schäfer, Bernd / Bernd Six: Sozialpsychologie des Vorurteils, Stuttgart u.a. 1978. 
Schenk, Michael: Medienwirkungsforschung, Tübingen 1987.

Schmidt, Siegfried J.: Die Wirklichkeit des Beobachters, in: Klaus Merten / Siegfried J. Schmidt / Weischenberg (1994), S. 3-19.

Schmitt, Manfred: Schönheit und Talent: Untersuchungen zum Verschwinden des Halo-Effekts, in: Zeitschrift für Experimentelle und Angewandte Psychologie 39/1992, S. 475-492.

Schneider, Wolf (Hrsg.): Unsere tägliche Desinformation. Wie die Massenmedien uns in die Irre führen, Hamburg 1984.

Schoeps, Julius H. (Hrsg.): Der politische Skandal, Stuttgart u.a. 1992.

Schröter, Detlef: Mitteilungs-Adäquanz. Studien zum Fundament eines realitätsgerechten journalistischen Handelns, in: Hans Wagner (Hrsg.): Idee und Wirklichkeit des Journalismus, München 1988, S. 175-216.

Schröter, Detlef: Qualität und Journalismus. Theoretische und praktische Grundlagen journalistischen Handelns, München 1995.

Schütz, Alfred: Der gut informierte Bürger. Ein Versuch über die soziale Verteilung des Wissens, in: ders.: Gesammelte Aufsätze, Band 2: Studien zur soziologischen Theorie, hrsg. v. Arvid Brodersen, Den Haag 1972, S. 85-101.

Schütz, Artur: Der Grubenhund. Experimente mit der Wahrheit, hrsg. v. Walter Hömberg, München 1996. [Erstausgabe 1931.]

Schütz, Astrid: Mehr oder weniger zugeben: Stufen defensiver Selbstdarstellung, in: Heinz-Ulrich Kohr / Massimo Martini / Angelika Kohr (Hg.): Macht und Bewußtsein. Europäische Beiträge zur Politischen Psychologie, Weinheim 1990, S. 131-141.

Schütz, Astrid: Selbstdarstellung von Politikern. Analyse von Wahlkampfauftritten, Weinheim 1992.

Schütz, Astrid: Politik oder Selbstdarstellung? Beispiele von Politikerauftritten, in: Michael Jäckel / Peter Winterhoff-Spurk (Hg.): Politik und Medien. Analysen zur Entwicklung der politischen Kommunikation, Berlin 1994, S. 193-209.

Schütze, Christian: Skandal. Eine Psychologie des Unerhörten, Bern/München ${ }^{2} 1985$.

Schuller, Wolfgang: Frevel, Raub, Bestechung - Volksversammlung und Senat. Skandale und Öffentlichkeit in der griechischen und römischen Welt, in: Rolf Ebbinghausen / Sighard Neckel (1989), S. 83-103, S. 83.

Schulman, Gary I. / Chrysoula Worrall: Salience patterns, Source Credibility, and the Sleeper Effect, in: Public Opinion Quarterly 34/1970, S. 371-382.

Schulz, Winfried (Hrsg.): Medienwirkungen. Einflüsse von Presse, Radio und Fernsehen auf Individuum und Gesellschaft. Untersuchungen im Schwerpunktprogramm "Publizistische Medienwirkungen", Weinheim 1992.

Schum, David A.: Knowledge, Probability, and Credibility, in: Journal of Behavioral Decision Making 2/1989, S. 39-62.

Schuster, Martin: Gesichtsschönheit: Begriff, Geschichte und Merkmale, in: Hassebrauck / Niketta (1993), S. 11-28. 
Schweitzer, Don A.: A Note on Whitehead's 'Factors of Source Credibility', in: Quarterly Journal of Speech 55/1969, S. 308-310.

Schweitzer, Don A.: The Effect of Presentation on Source Evaluation, in: Quarterly Journal of Speech 56/1970, S. 33-39.

Schweitzer, Don / Gerald P. Ginsburg: Factors of Communicator Credibility, in: Carl W. Backman / Paul F. Secord (Hg.): Problems in Social Psychology. Selected Readings, New York u.a. 1966, S. 94-102.

Sereno, Kenneth K.: Ego-Involvement, High Source Credibility, and Response to a Belief-Discrepant Communication, in: Speech Monographs 35/1968, S. 476-481.

Sereno, Kenneth K. / Gary J. Hawkins: The Effects of Variations in Speaker's Nonfluency upon Audience Ratings Toward the Speech Topic and Speaker's Credibility, in: Quarterly Journal of Speech 50/1964, S. 36-44.

Sereno, Kenneth K. / Gary J. Hawkins: The Effects of Variations in Speaker's Nonfluency upon Audience Ratings Toward the Speech Topic and Speaker's Credibility, in: Speech Monographs 34/1967, S. 58-64.

Severin, Werner J. / James W. Tankard, Jr.: Communication Theories: Origins, Methods, And Uses In The Mass Media, New York ${ }^{3} 1992$.

Settle, Robert B. / Linda L. Golden: Attribution Theory and Advertiser Credibility, in: Journal of Marketing Research 9/1974, S. 181-185.

Sharp, Harry Jr. / Thomas McClung: Effects of Organization on the Speaker's Ethos, in: Speech Monographs 33/1966, S. 182-183.

Shaw, Eugene F.: Media Credibility: Taking The Measure of a Measure, in: Journalism Quarterly 50/1973, S. 306-311.

Shaw, John: Some "Real-Life" Accounts of Influential Relationships, in: Human Relations 30/1977, S. 363-372.

Sherif, Carolyn W. et al.: Personal Involvement, Social Judgment, and Action, in: Journal of Personality and Social Psychology 27/1973, S. 311-328.

Sherif, Carolyn W. / Muzafer Sherif: Attitude, Ego-Involvement and Change, New York 1967.

Siegel, Elliot R. / Gerald R. Miller / C. Edward Wotring: Source Credibility and Credibility Proneness. A New Relationship, in: Speech Monographs 36/1969, S. 118-125.

Sigall, Harold / Elliot Aronson: Liking for an Evaluator as a Function of Her Physical Attractiveness and Nature of the Evaluations, in: Journal of Experimental Social Psychology 5/1969, S. $93-$ 100.

Sigall, Harold / Robert Helmreich: Opinion Change as a Function of Stress and Communicator Credibility, in: Journal of Experimental Social Psychology 5/1969, S. 70-78.

Simons, Herbert W. / Nancy N. Berkowitz / R. John Moyer: Similarity, Credibility, and Attitude Change: A Review and a Theory, in: Psychological Bulletin 73/1970, S. 1-16. 
Singletary, Michael W.: Components of Credibility of a Favourable News Source, in: Journalism Quarterly 53/1976, S. 316-319.

Singletary, Michael W. / Kevin Carragee: Subjective Errors in Written Messages, in: Journalism Quarterly 57/1980, S. 432-437.

Sinnett, E. Robert / Kenneth Hagen / William M. Harvey: Credibility of Sources of Information about Drugs to Heroin Addicts, in: Psychological Reports 37/1975, S. 1239-1242.

Sinnett, E. Robert et al.: Credibility of Sources and Information about Drugs, in: Psychological Reports 36/1975, S. 299-309.

Smith, Anthony J.: Similarity of Values and Its Relation to Acceptance and the Projection of Similarity, in: Journal of Psychology 43/1957, S. 251-260.

Smith, B. L. et al.: Effects of Speech Rate on Personality Perception, in: Language and Speech $18 / 1975$, S. 145-152.

Smith, George Horsley: Beliefs in Statements Labeled Fact and Rumor, in: Journal of Abnormal and Social Psychology 42/1947, S. 80-90.

Smith, Raymond G.: Source Credibility Context Effects, in: Speech Monographs 40/1973, S. 303309.

Snyder, Mark / Myron Rothbart: Communicator Attractiveness and Opinion Change, in: Canadian Journal of Behavioral Science 3/1971, S. 377-387.

Sobczak, Patrick M. / Michael R. Bowers: An Experimental Design for Measuring the Effects of Source Credibility and Goal Behavior Estimate in Health Promotion Television Commercials, in: Psychology. A Journal of Human Behavior 30/1993, S. 30-43.

Speck, Paul Surgi: On Humor and Humor in Advertising. Unpublished Doctoral Dissertation, Texas Tech University 1987.

Stamm, Keith / Ric Dube: The Relationship of Attitudinal Components to Trust in Media, in: Communication Research 21/1994, S. 105-123.

Stanley, Thomas J.: Are Highly Credible Sources Persuasive? In: Journal of Consumer Research 5/1978, S. 66-67.

Starck, Kenneth: Media Credibility in Finland: a Cross-National Approach, in: Journalism Quarterly 46/1969, S. 790-795.

Starkulla, Heinz: Publizistik und Kommunikation. Ein Beitrag zur wissenschaftlichen Erkenntnis der kommunikativen Wirklichkeit, in: Festschrift für Hanns Braun, Bremen 1963, S. 158-167.

Starkulla, Heinz: Marktplätze sozialer Kommunikation. Bausteine einer Medientheorie, München 1993.

Steinfatt, Thomas M.: A Criticism of 'Dimensions of Source Credibility: A Test for Reproducibility', in: Speech Monographs 41/1974, S. 291-292.

Steinmann, Matthias F.: Massenmedien und Werbung, Freiburg i.Br. 1971.

Sternthal, Brian / Ruby Roy Dholakia: Rejoinder to "Are Highly Credible Sources Persuasive?" In: 
Journal of Consumer Research 5/1978, S. 67-69.

Sternthal, Brian / Ruby Dholakia / Clark Leavitt: The Persuasive Effect of Source Credibility: Tests of Cognitive Response, in: Journal of Consumer Research 4/1978, S. 252-260.

Sternthal, Brian / Lynn W. Phillips / Ruby Dholakia: The Persuasive Effect of Source Credibility: A Situational Analysis, in: Public Opinion Quarterly 42/1978, S. 285-314.

Stiff, James B.: Cognitive Processing of Persuasive Message Cues: A Meta-Analytic Review of the Effects of Supporting Information on Attitudes, in: Communication Monographs 53/1986, S. 75-89.

Stoltenberg, Cal D. / Carl S. Davis: Career and Study Skills Information: Who says What Can Alter Message Processing, in: Journal of Social and Clinical Psychology 6/1988, S. 38-52.

Stone, Vernon A.: Individual Differences and Inoculation Against Persuasion, in: Journalism Quarterly 46/1969, S. 267-273.

Stone, Vernon A.: Sources of Most News: Evidence and Inference, in: Journal of Broadcasting 14/1969-70, S. 1-4.

Stone, Vernon A. / Steven H. Chaffee: Familiy Communication Patterns and Source-Message Orientation, in: Journalism Quarterly 47/1970, S. 239-246.

Stone, Vernon A. / Harrogadde S. Eswara: The Likability and Self-Interest of the Source in Attitude Change, in: Journalism Quarterly 46/1969, S. 61-68.

Stone, Vernon A. / James L. Hoyt: Source-Message Orientation and Expectation of Discussion with the Source, in: Journalism Quarterly 48/1971, S. 741-744.

Stone, Vernon A. / James L. Hoyt: Effect of Likability and Relevance of Expertness, in: Journalism Quarterly 51/1974, S. 314-317. [1974a]

Stone, Vernon A. / James L. Hoyt: The Emergence of Source-Message Orientation as a Communication Variable, in: Communication Research 1/1974, S. 89-109. [1974b]

Stotland, Ezra / Daniel Katz / Martin Patchen: The Reduction of Prejudice through the Arousal of Self-Insight, in: Journal of Personality 27/1959, S. 507-531.

Stotland, Ezra / Martin Patchen: Identification and Changes in Prejudice and in Authoritarianism, in: Journal of Abnormal and Social Psychology 62/1961, S. 265-274.

Strack-Zimmermann, Marie-Agnes: Bilder aus Amerika. Eine zeitungswissenschaftliche Studie über die USA-Berichterstattung im Zweiten Deutschen Fernsehen (ZDF), Frankfurt a.M. 1987.

Straßmann, Burghard: Gut gefälscht ist halb gewonnen, in: Die Zeit vom 02.02.1996.

Stroebe, Wolfgang et al.: Effects of Physical Attractiveness, Attitude Similarity, and Sex on Various Aspects of Interpersonal Attraction, in: Journal of Personality and Social Psychology 18/1971, S. 79-91.

Sunnafrank, Michael: Interpersonal Attraction and Attitude Similarity: A Communication-Based Assessment, in: James A. Anderson (Hrsg.): Communication Yearbook 14, Newbury Park u.a. 1991, S. 451-483.

Swenson, R. A. / D. L. Nash / D. C. Roos: Source Credibility and Perceived Expertness of 
Testimony in a Simulated Child Custody Case, in: Professional Psychology: Research and Practice 15/1984, S. 891-898.

Szasz, Andrew: The Process and Significance of Political Scandals: A Comparison of Watergate and the "Sewergate" Episode at the Environmental Protection Agency, in: Social Problems 33/1986, S. 202-217.

Szewczyk, Hans / Eckhard Littmann: Untersuchungen zur Glaubwürdigkeitsbeurteilung kindlicher Zeugen, in: Trankell (1982), S. 75-103.

Tannenbaum, Percy H.: Initial Attitude Toward Source and Concept as Factors in Attitude Change Through Communication, in: Public Opinion Quarterly 20/1956, S. 413-425.

Tardy, Charles H.: Interpersonal Evaluations: Measuring Attraction and Trust, in: ders. (Hrsg.): A Handbook for the Study of Human Communication: Methods and Instruments for Observing, Measuring, and Assessing Communication Processes, Norwood, N.J. 1988, 269-283.

Teichert, Will: Tschernobyl in den Medien. Ergebnisse und Hypothesen zur Tschernobyl-Berichterstattung, in: Rundfunk und Fernsehen 35/1987, S. 185-204.

Tesser, Abraham: Evaluative and Structural Similarity of Attitudes as Determinants of Interpersonal Attraction, in: Journal of Personality and Social Psychology 18/1971, S. 92-96.

Tiemens, Robert K.: Some Relationships of Camera Angle to Communicator Credibility, in: Journal of Broadcasting 14/1970, S. 483-490.

Tokheim, C. L. / J.[ohn] W. Wanzenried / F. C. Powell: Cigarette Smoking: Effect on Perceptions of Source Credibility, in: Psychological Reports 66/1990, S. 1388-1390.

Topalova, Velina: Credibility of Information Source, in: Polish Psychological Bulletin 5/1974, S. 7380 .

Touhey, Catherine E.: Prior Information, Credibility, and Attitude Change, in: Journal of Social Psychology 95/1975, S. 287-288.

Trankell, Arne: Zur Methodik der Glaubwürdigkeitsuntersuchungen, in: Psychologie und Praxis 1/1956-57, S. 292-312.

Trankell, Arne: Der Realitätsgehalt von Zeugenaussagen. Methodik der Aussagepsychologie, Göttingen 1971.

Trankell, Arne (Hrsg.): Reconstructing the Past. The Role of Psychology in Criminal Trials, Stockholm 1982.

Tucker, Raymond K.: Reliability of Semantic Differential Scales: The Role of Factor Analysis, in: Western Speech 35/1971, S. 185-190.

Tuppen, Christopher J. S.: Dimensions of Communicator Credibility: An Oblique Solution, in: Speech Monographs 41/1974, S. 253-260.

Tybout, Alice M.: Relative Effectiveness of Three Behavioral Influence Strategies as Supplements to Persuasion in a Marketing Context, in: Journal of Marketing Research 15/1978, S. 229-242. 
Uenk, Renate / Susanne Laarmann: Medium Zeitung. Vergleichende Darstellung und Analyse von Werbeträgern, Frankfurt a.M. 1992.

Undeutsch, Udo: Beurteilung der Glaubhaftigkeit von Aussagen, in: ders. (Hrsg.): Handbuch der Psychologie Bd. 11: Forensische Psychologie, Göttingen 1967, S. 26-181.

Undeutsch, Udo: Statement Reality Analysis, in: Trankell (1982), S. 27-56.

Undeutsch, Udo: Courtroom Evaluation of Eyewitness Testimony, in: International Review of Applied Psychology 33/1984, S. 51-67.

U.St.: Tugend-TV, in: Die Zeit vom 17.11.1995.

Völzing, Paul-Ludwig: Begründen, Erklären, Argumentieren. Modelle und Materialien zu einer Theorie der Metakommunikation, Heidelberg 1979.

Vrij, Aldert: Credibility Judgments of Detectives: The Impact of Nonverbal Behavior, Social Sills, and Physical Characteristics on Impression Formation, in: Journal of Social Psychology 133/1993, S. 601-610.

Wagner, Hans: Vermittlungsverfassung in der Massenkommunikation, in: Publizistik 22/1977, S. 513.

Wagner, Hans: Kommunikation und Gesellschaft, Band 1, München 1978.

Wagner, Hans: Rationalisierungsprozesse der Sozialen Kommunikation, in: Politische Bildung 13/1980, S. 3-32.

Wagner, Hans: Wirklichkeits-Präparate? Das Medikamenten-Bild in deutschen Printmedien, in: Hans Wagner / Heinz Starkulla (Hg.): Medizin \& Medien. Krankt die Gesundheit am Journalismus? München 1984, S. 10-104.

Wagner, Hans: Der Name des Fortschritts heißt 'Erfolg', München 1986 (unveröffentlichtes Manuskript).

Wagner, Hans: Der schlecht informierte Bürger. Zur Phänomenologie des Informationsverhaltens und dessen möglicher Steuerung. Festvortrag zur Akademischen Feier für Heinz Starkulla am 18. Februar 1988, o.O. (unveröffentlichtes Manuskript).

Wagner, Hans: KommunikationsWissenschaft (ZeitungsWissenschaft), München ${ }^{2} 1989$.

Wagner, Hans: Medien-Tabus und Kommunikationsverbote. Die manipulierbare Wirklichkeit, München 1991

Wagner, Hans: Journalismus I: Auftrag. Gesammelte Beiträge zur Journalismustheorie, Erlangen 1995.

Wagner, Hans: Das Fach-Stichwort: Das Grubenhund-Gesetz: Die Rationalität der sozialen Orientierung, in: Schütz (1996), S. 119-192.

Wagner, Hans (Hrsg.): Idee und Wirklichkeit des Journalismus, München 1988.

Wagner, Hans / Heinz Starkulla (Hg.): Medizin \& Medien. Krankt die Gesundheit am Journalismus? München 1984.

Waisanen, F.B. / Jerome T. Durlak: Mass Media Use, Information Source Evaluation, and Perceptions of Self and Nation, in: Public Opinion Quarterly 31/1967, S. 399-406. 
Wakshlag, Jacob J. / Nadyne G. Edison: Attraction, Credibility, Perceived Similarity, and the Image of Public Figures, in: Communication Quarterly 27/1979, S. 27-34.

Walster, Elaine / Elliot Aronson / Darcy Abrahams: On Increasing the Persuasiveness of a Low Prestige Communicator, in: Journal of Experimental Social Psychology 2/1966, S. 325-342.

Walster, Elaine / Leon Festinger: The Effectiveness of "Overheard" Persuasive Communications, in: Journal of Abnormal and Social Psychology 65/1962, S. 395-402.

Wanta, Wayne / Yu-Wei Hu: The Effects of Credibility, Reliance, and Exposure on Media AgendaSetting: A Path Analysis Model, in: Journalism Quarterly 71/1994, S. 90-98.

Wanzenried, J[ohn]. W. / D. Smith-Howell / F. C. Powell: Source Credibility and Presidential Campaigns: Governor Clinton and the Allegation of Marital Infidelity, in: Psychological Reports 70/1992, S. 992-994.

Wanzenried, J[ohn] W. / F. C. Powell: Source Credibility and Dimensional Stability: A Test of the Leathers Personal Credibility Scale Using Perceptions of Three Presidential Candidates, in: Perceptual and Motor Skills 77/1993, S. 403-406.

Ward, Charles D. / Elliott McGinnies: Persuasive Effects of Early and Late Mention of Credible and Noncredible Sources, in: Journal of Psychology 86/1974, S. 17-23.

Ware, P. Dale / Raymond K. Tucker: Heckling as Distraction: An Experimental Study of Its Effect on Source Credibility, in: Speech Monographs 41/1974, S. 185-188.

Warren, Irving D.: The Effect of Credibility in Sources of Testimony on Audience Attitudes toward Speaker and Message, in: Speech Monographs 36/1969, S. 456-458.

Wegner, Daniel M. et al.: Incrimination Through Innuendo: Can Media Questions Become Public Answers? In: Journal of Personality and Social Psychology 40/1981, S. 822-832.

Wegrocki, Henry J.: The Effect of Prestige Suggestibility on Emotional Attitudes, in: Journal of Social Psychology 5/1934, S. 384-394.

Weick, Karl E. / David P. Gilfillan / Thomas A. Keith: The Effect of Composer Credibility on Orchestra Performance, in: Sociometry 36/1973, S. 435-462.

Weinberger, Marc G. / Charles S. Gulas: The Impact of Humor in Advertising: A Review, in: Journal of Advertising 21/1992, S. 35-59.

Weischenberg, Siegfried: Bei diesen Zeitungsschreibern lese ich alles zweimal. Was Journalistenimages mit Medienwirkungen zu tun haben, in: Pürer (1985), S. 80-106.

Weischenberg, Siegfried: Die Glaubwürdigkeitslücke des Fernsehjournalismus. Anmerkungen zum Zusammenhang zwischen der Politikmüdigkeit der Bevölkerung und der aktuellen politischen Berichterstattung, in: Media Perspektiven 11/1987, S. 711-717.

Weischenberg, Siegfried: Nachrichtenschreiben. Journalistische Praxis zum Studium und Selbststudium, Opladen ${ }^{2} 1990$.

Weiss, Walter: A Sleeper Effect in Opinion Change, in: Journal of Abnormal and Social Psychology 48/1953, S. 173-180.

Weiss, Walter: Opinion Congruence with a Negative Source on One Issue as a Factor Influencing 
Agreement on Another Issue, in: Journal of Abnormal and Social Psychology 54/1957, S. 180-186.

Weiss, Walter / Bernard J. Fine: The Effect of Induced Agressiveness on Opinion Change, in: Journal of Abnormal and Social Psychology 52/1956, S. 109-114.

Wessell, Nils Hartley: The Credibility, Impact and Effectiveness of the Soviet General Press: An Analysis of Soviet Research on the Soviet Non-Specialized Newspaper, Ann Arbor 1973.

West, Mark Douglas: Validating a Scale for the Measurement of Credibility: A Covariance Structure Modelling Approach, in: Journalism Quarterly 71/1994, S. 159-168.

Westley, Bruce H. / Werner J. Severin: Some Correlates of Media Credibility, in: Journalism Quarterly 41/1964, S. 325-335.

Wheeless, Lawrence R.: Attitude and Credibility in the Prediction of Attitude Change: A Regression Approach, in: Speech Monographs 41/1974, S. 277-281. [1974a]

Wheeless, Lawrence R.: The Effects of Attitude, Credibility, and Homophily on Selective Exposure to Information, in: Speech Monographs 41/1974, S. 329-338. [1974b]

Wheeless, Lawrence R.: A Follow-Up Study of the Relationships Among Trust, Disclosure, and Interpersonal Solidarity, in: Human Communication Research 4/1978, S. 143-157.

Wheeless, Lawrence R. / Janis Grotz: The Measurement of Trust and its Relationship to SelfDisclosure, in: Human Communication Resarch 3/1977, S. 250-257.

Whitehead, Jack L., Jr.: Factors of Source Credibility, in: Quarterly Journal of Speech 54/1968, S. 59-63.

Whitehead, Jack L., Jr.: Effects of Authority-Based Assertion on Attitude and Credibility, in: Speech Monographs 38/1971, S. 311-315.

Whittaker, James O.: Sex Differences and Susceptibility to Interpersonal Persuasion, in: Journal of Social Psychology 66/1965, S. 91-94.

Whittaker, James O. / Robert D. Meade: Sex of the Communicator as a Variable in Source Credibility, in: Journal of Social Psychology 72/1967, S. 27-34.

Whittaker, James O. / Robert D. Meade: Retention of Opinion Change as a Function of Differential Source Credibility. A Cross-Cultural Study, in: International Journal of Psychology 3/1968, S. 103-108.

Wickert, Ulrich: Eine Frage der Qualität. Aktuelle Information im Ersten, in: Arbeitsgemeinschaft der öffentlich-rechtlichen Rundfunkanstalten der Bundesrepublik Deutschland (ARD) (Hrsg.): ARD Jahrbuch 1995, Hamburg 1995, S. 120-123.

Wiegmann, O.: Two Politicians in a Realistic Experiment: Attraction, Discrepancy, Intensity of Delivery, and Attitude Change, in: Journal of Applied Social Psychology 15/1985, S. 673686.

Wild, Wolfgang: Kriterien der Glaubwürdigkeit von Expertenaussagen. Vortrag bei der öffentlichen Jahrestagung des Vereins der Freunde der Universität Regensburg e.V. am 29. Juli 1987 in 
Regensburg, o.O., o.J.

Wilkins, Lee / Philipp Patterson: Risk Analysis and the Construction of News, in: Journal of Communication 37/1987, S. 80-92.

Wilson, C. Edward / Douglas M. Howard: Public Perception of Media Accuracy, in: Journalism Quarterly 55/1978, S. 73-76.

Winiarski, Rolf: Das Gesetz der Gesetzlosen. Zur Dynamik des Skandals am Beispiel der Kieler CDU-Affäre, in: Moser (1989), S. 52-65.

Winkler, Hans-Joachim: Über die Bedeutung von Skandalen für die politische Bildung, in: Aus Politik und Zeitgeschichte (Beilage zu 'Das Parlament') 27/1968, S. 3-14.

Woodside, Arch G. / J. William Davenport: The Effect of Salesman Similarity and Expertise on Consumer Purchasing Behavior, in: Journal of Marketing Research 11/1974, S. 198-202.

Worchel, Stephen / Virginia A. Andreoli / J. Eason: Is the Medium the Message: A Study of the Effects of Media, Communicator, and Message Characteristics on Attitude Change, in: Journal of Applied Spcial Psychology 5/1975, S. 157-172.

Wright, Thomas L. / Jack Arbuthnot / Robert Silber: Interpersonal Trust and Attributions of Source Credibility: Evaluations of a Political Figure in a Crisis, in: Perceptual and Motor Skills 44/1977, S. 943-950.

Wu, Chenghuan / David R. Shaffer: Susceptibility to Persuasive Appeals as a Function of Source Credibility and Prior Experience With the Attitude Object, in: Journal of Personality and Social Psychology 52/1987, S. 677-688.

Wyer, Robert S., Jr. / Sandra Schwartz: Some Contingencies in the Effects of the Source of a Communication on the Evaluation of that Communication, in: Journal of Personality and Social Psychology 11/1969, S. 1-9.

Zanna, Mark P. / Ellen C. Klosson / John M. Darley: How Television News Viewers Deal with Facts that Contradict their Beliefs: A Consistency and Attribution Analysis, in: Journal of Applied Social Psychology 6/1976, S. 159-176.

Zanna, Mark P. / James M. Olson / C. Peter Herman (Hg.): Social Influence: The Ontario Symposium, Vol. 5, Hillsdale, NJ. 1987.

Zanna, Mark P. / Steven M. del Veccio: Perceived Credibility of Television News: A Matter of Viewer's Attitudes and the Position taken by the Media, in: European Journal of Social Psychology 3/1973, S. 213-216.

Zhu, Jian-Hua: Information Availability, Source Credibility and Audience Sophistication: Factors Conditioning the Effects of Communist Propaganda in China. Unpublished Doctoral Dissertation, Indiana University 1990.

Zimmerli, Walter Ch. / Hansjörg Sinn (Hg.): Die Glaubwürdigkeit technisch-wissenschaftlicher Experten, Düsseldorf 1990.

Zschunke, Peter: Agentur-Journalismus. Nachrichtenschreiben im Sekundentakt, München 1994. 
Zuckerman, Miron et al.: Facial and Vocal Cues of Deception and Honesty, in: Journal of Experimental Social Psychology 15/1979, S. 378-396.

Zuckerman, Miron / Bella M. DePaulo / Robert Rosenthal: Verbal and Nonverbal Communication of Deception, in: Leonard Berkowitz (Hrsg.): Advances in Experimental Social Psychology, Vol. 14, New York/London 1981, S. 1-59.

Zuckerman, Miron / R. Koestner / R. Driver: Beliefs about Cues Associated with Deception, in: Journal of Nonverbal Behavior 6/1981, S. 105-114. 